\title{
A Versatile Chemoenzymatic Synthesis for the Discovery of Potent Cryptophycin Analogues
}

Jennifer J. Schmidt ${ }^{1,2}$, Yogan Khatri ${ }^{1}$, Scott I. Brody ${ }^{1}$, Catherine Zhu ${ }^{1,3}$, Halina Pietraszkiewicz ${ }^{6}$, Frederick A. Valeriote ${ }^{6}$, David H. Sherman ${ }^{1,2,3,4,5^{*}}$

${ }^{1}$ Life Sciences Institute, ${ }^{2}$ Department of Medicinal Chemistry, ${ }^{3}$ Program in Chemical Biology, ${ }^{4}$ Department of Chemistry, ${ }^{5}$ Department of Microbiology \& Immunology, University of Michigan, Ann Arbor, Michigan 48109, United States

${ }^{6}$ Department of Internal Medicine, Henry Ford Cancer Institute, Henry Ford Health System, Detroit, Michigan 48202, United States 
Title Page

Table of Contents

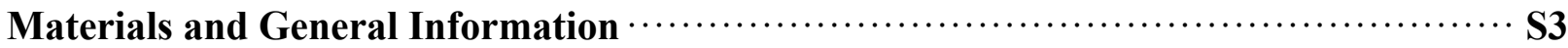

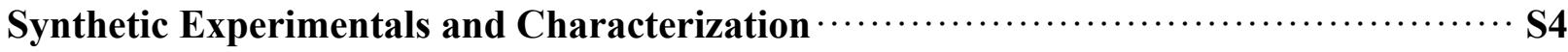

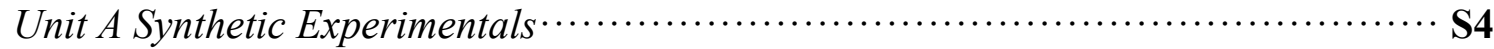

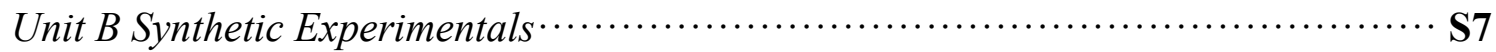

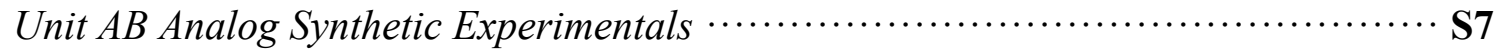

Unit CD Synthetic Experimentals …......................................... S11

Seco-Cryptophycin Analog Synthetic Experimentals ……....................... S12

Biocatalytic Elaboration of Cryptophycin Analogs …….......................... S18

General Cloning, Protein Expression, Purification and Analysis of CrpTE and CrpE $\cdots$ S25

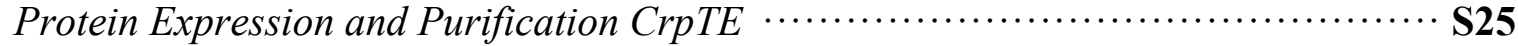

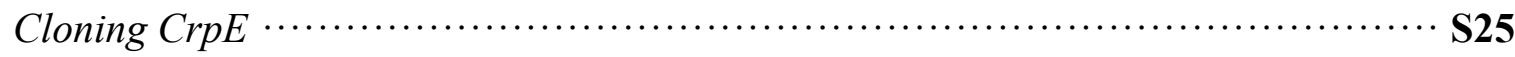

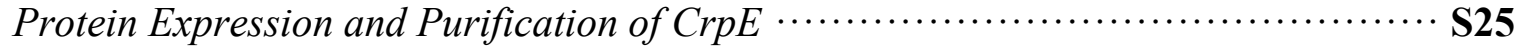

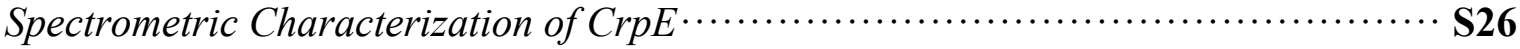

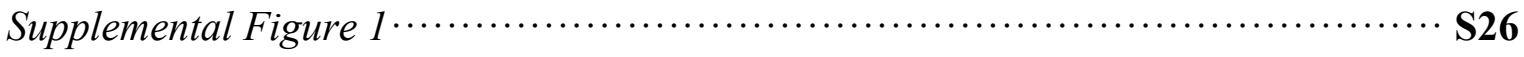

Spin-state Shift and Substrate Dissociation Constant Determination CrpE $\cdots \cdots \cdots \cdots \cdots . \cdots . .627$

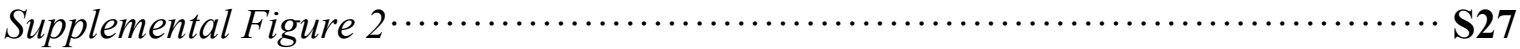

Analytical Evaluation of Cryptophycin Analogs ……........................... S28

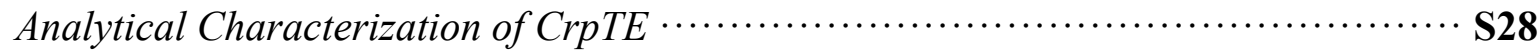

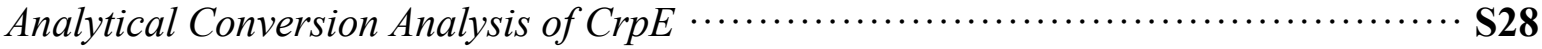

LCMS analysis of Analytical CrpE Conversion ……….......................... S28

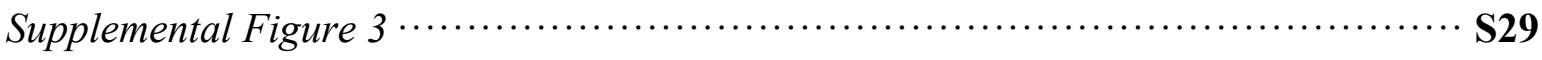

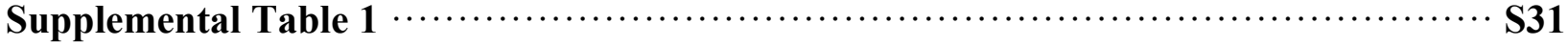

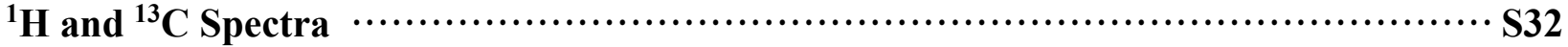

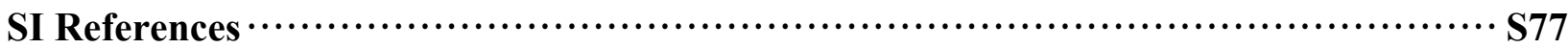




\section{Materials and General Information}

Unless otherwise noted, chemical reagents and solvents were purchased from EMD Millipore, SigmaAldrich, Oakwood, Combi blocks, Chem Impex, and Thermo-Fisher Scientific. Kanamycin sulfate and isopropyl- $\beta$-D-thiogalactopyranoside (IPTG) were obtained from Gold Biotechnology. Lysozyme was purchased from RPI. Imidazole was purchased from AK Scientific. Amicon Ultra centrifugal filters and PD10 desalting columns used for protein concentration and buffer exchange were purchased from GE Healthcare.

Deionized water was obtained from a Milli-Q system (EMD Milipore) using Q-Gard 2/Quantum Ex Ultrapure organex cartridges. Media components for E. coli growth were purchased from EMD Milipore, SigmaAldrich, and Thermo-Fisher Scientific. Glycerol was purchased from BDH via VWR. LB broth and LB agar (Miller) were purchased in pre-made granulated form from EMD Millipore. TB broth was made from individually purchased components and consisted of $4 \% \mathrm{v} / \mathrm{v}$ glycerol. Media and solutions were autoclaved or sterile filtered before use. For all solutions, $\mathrm{pH}$ was monitored using a VWR symphony SB 70P pH meter calibrated according to the manufacturer's specifications.

Chemically competent $E$. coli $\mathrm{BI} 21$ (DE3) and C43(DE3) were used for over expression of protein. These were prepared as per the MixandGo buffer kit purchased from Zymo Research. Optical density $\left(\mathrm{OD}_{600}\right)$ was measured using an Eppendorf BioPhotometer.

Unless otherwise noted, all reactions were performed in flame dried glass wear under an atmosphere of dry nitrogen. Reactions run at elevated temperatures were controlled by IKA RET control visc (model RS $232 \mathrm{C}$ ), reactions run at room temperature (rt) was $\sim 21-23^{\circ} \mathrm{C}$, reactions run cooler than room temperature were performed in an ice bath $\left(0^{\circ} \mathrm{C}\right)$, dry ice/acetone $\left(-78^{\circ} \mathrm{C}\right)$, or isopropanol/ThermoNESLAB (model $\mathrm{CC} 100)$ for extended times/other temperatures. Commercially available starting materials and reagents were used as received unless otherwise noted. Dichloromethane was purchased as HPLC grade from fisher and used directly. Tetrahydrofuran, N,N Dimethylformamide, and pyridine were purchased as anhydrous and unstabilized (EMD Millipore DriSolv). Acetone was dried over $\mathrm{Na}_{2} \mathrm{SO}_{4}$ prior to use for reactions. Diisopropylamine and trimethylamine were distilled from calcium hydride directly prior to use.

${ }^{1} \mathrm{H}$ NMR spectra were recorded on either a Varian 600 NMR System $(600 \mathrm{MHz})$ or Varian 400-MR (400 $\mathrm{MHz}$ ) spectrometer. Chemical shifts are reported in parts per million (ppm) using the solvent resonance as an internal standard $\left(\mathrm{CDCl}_{3} 7.26\right.$ and $\left.\mathrm{CD}_{3} \mathrm{OD} 3.31 \mathrm{ppm}\right)$. Data are reported as follows: chemical shift, multiplicity $(\mathrm{s}=$ singlet, $\mathrm{d}=$ doublet, $\mathrm{t}=$ triplet, $\mathrm{q}=$ quartet, $\mathrm{p}=$ pentet, $\mathrm{h}=$ hextet), coupling constant $(\mathrm{Hz})$, and integration. Proton decoupled ${ }^{13} \mathrm{C}$ NMR spectra were recorded on varian $600 \mathrm{NMR}$ System $(150 \mathrm{MHz})$ or Varian 400-MR (100 MHz) spectrometer. Chemical shifts are reported in ppm using relative to residual solvent peaks $\left(\mathrm{CDCl}_{3}, 77.0 \mathrm{ppm}\right.$ or $\mathrm{CD}_{3} \mathrm{OD}$ 49.0). High resolution mass spectra as well as analytical reaction analysis was performed on an Agilent Technologies 6250 TOF LC/MS equipped with an Agilent 1290 infinity II HPLC (monitored at $235 \mathrm{~nm}$ ) at the Life Sciences Institute at the University of Michigan. Preparative scale reactions were purified utilizing a Shimadzu LC 20AT prominence liquid chromatography system with a diode array detector.

Analytical Thin layer chromatography was performed on EMD Millipore $0.25 \mathrm{~mm}$ silica gel $F_{254}$ plates. Visualization was accomplished by a combination of $254 \mathrm{~nm}$ UV lamp and either potassium permanganate $\left(\mathrm{KMNO}_{4}\right)$, ceric ammonium molybdate $(\mathrm{CAM})$, or $\mathrm{p}$-anisaldehyde. Purifications were performed by forced air flash chromatography using EMD Millipore Silica Gel 60 (40 - 63 um) or a Biotage Isolera one flash purification system. Columns used with Biotage Isolera one purification system include Biotage SNAP Ultra, SiliaSep, SiliaSepHP, and SiliaSep amine cartridges. 


\section{Synthetic Experimentals and Characterization}

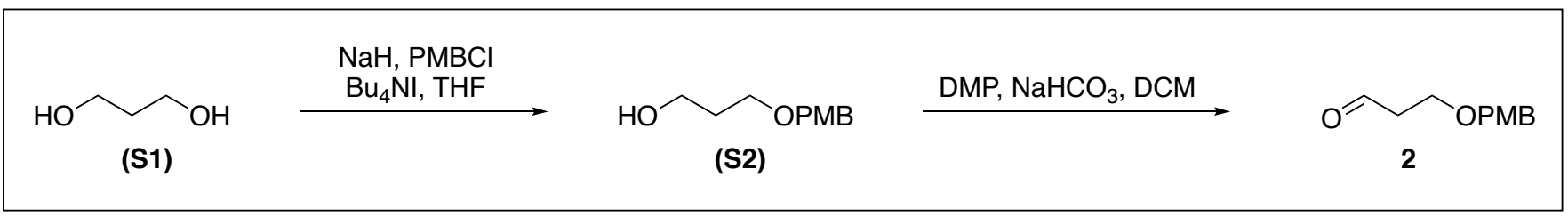

\section{Unit A Synthetic Experimentals}

1 was synthesized as reported previously, all spectra were in accordance with previous literature. ${ }^{l}$

$\mathbf{2}$ was synthesized over two steps from commercially available S1, all spectra were in accordance with previous literature. ${ }^{2,3}$

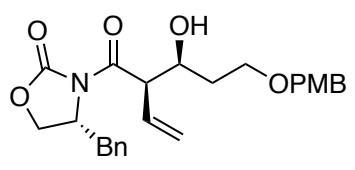

(R)-4-benzyl-3-((2R,3S)-3-hydroxy-5-((4-methoxybenzyl)oxy)-2vinylpentanoyl)oxazolidin-2-one (S3). To a three-neck flask with an internal temperature probe was added a solution of 1 (7.502 g, $30.58 \mathrm{mmol}, 1 \mathrm{eq})$ in $\mathrm{CH}_{2} \mathrm{Cl}_{2}$ $(305 \mathrm{~mL}, 0.1 \mathrm{M})$ and cooled to $-78{ }^{\circ} \mathrm{C}$. This was treated with dibutylboron trifluoromethanesulfonate (1 M in DCM, $33.64 \mathrm{~mL}, 33.64 \mathrm{mmol}, 1.1 \mathrm{eq})$. After 5 minutes, the mixture was treated with $\mathrm{Et}_{3} \mathrm{~N}(6.02 \mathrm{~mL}, 42.8 \mathrm{mmol}, 1.4 \mathrm{eq})$, then kept for $1 \mathrm{~h}$ at $-78{ }^{\circ} \mathrm{C}, 20$ minutes at $0{ }^{\circ} \mathrm{C}$, and recooled to $-78{ }^{\circ} \mathrm{C}$. At this point a solution of aldehyde $2(8.31 \mathrm{~g}, 42.81 \mathrm{mmol}, 1.4 \mathrm{eq})$ in $\mathrm{CH}_{2} \mathrm{Cl}_{2}(25 \mathrm{~mL})$ was added dropwise. The reaction was kept for $1 \mathrm{~h}$ at $-78^{\circ} \mathrm{C}$, and $1 \mathrm{~h}$ at $0{ }^{\circ} \mathrm{C}$ before quenching with sodium phosphate buffer $(\mathrm{pH} 7,500 \mathrm{mM}, 30 \mathrm{~mL})$ followed by MeOH $(30 \mathrm{~mL})$. After 5 minutes, $30 \% \mathrm{H}_{2} \mathrm{O}_{2}(10 \mathrm{~mL})$ was added keeping the temperature $<10{ }^{\circ} \mathrm{C}$ and the mixture was stirred for at $0{ }^{\circ} \mathrm{C}$ for $1 \mathrm{~h}$. Organics were removed under reduced pressure and the remaining aqueous layer was extracted with EtOAc $(3 \mathrm{x} 100 \mathrm{~mL})$. The combined organics were washed with $1 \mathrm{~N} \mathrm{HCl}, 5 \%$ aq $\mathrm{NaHCO}_{3}$, brine, dried over $\mathrm{Na}_{2} \mathrm{SO}_{4}$, filtered, and concentrated. The residue was purified by flash chromatography (33\% EtOAc/Hexanes) to give S3 (11.98 g, $89 \%$ yield) as a clear and colorless oil: $\mathrm{R}_{\mathrm{f}}=0.2\left(33 \%\right.$ EtOAc/Hexanes); ${ }^{1} \mathbf{H} \mathbf{~ N M R}\left(600 \mathrm{MHz}, \mathrm{CDCl}_{3}\right) \delta$ 7.31 (ddd, J = 7.4, 6.4, 1.3 Hz, 2H), $7.28-7.25(\mathrm{~m}, 2 \mathrm{H}), 7.24-7.21(\mathrm{~m}, 2 \mathrm{H}), 7.20-7.15(\mathrm{~m}, 2 \mathrm{H}), 6.04$ (dddd, $\mathrm{J}=17.6,10.0,9.0,1.1 \mathrm{~Hz}, 1 \mathrm{H}), 5.39-5.36(\mathrm{~m}, 1 \mathrm{H}), 5.36-5.34(\mathrm{~m}, 1 \mathrm{H}), 4.68(\mathrm{ddt}, \mathrm{J}=11.4,6.3$, $3.0 \mathrm{~Hz}, 1 \mathrm{H}), 4.58-4.51(\mathrm{~m}, 1 \mathrm{H}), 4.42(\mathrm{~s}, 2 \mathrm{H}), 4.25-4.19(\mathrm{~m}, 1 \mathrm{H}), 4.18-4.11(\mathrm{~m}, 2 \mathrm{H}), 3.78(\mathrm{~d}, \mathrm{~J}=1.1$ $\mathrm{Hz}, 3 \mathrm{H}), 3.69-3.64(\mathrm{~m}, 1 \mathrm{H}), 3.64-3.56(\mathrm{~m}, 1 \mathrm{H}), 3.28-3.17(\mathrm{~m}, 1 \mathrm{H}), 2.74(\mathrm{dd}, \mathrm{J}=13.4,9.5 \mathrm{~Hz}, 1 \mathrm{H})$, $1.93-1.78(\mathrm{~m}, 1 \mathrm{H}), 1.74(\mathrm{dt}, \mathrm{J}=14.5,7.0 \mathrm{~Hz}, 1 \mathrm{H}), 1.55(\mathrm{~s}, 2 \mathrm{H}) ;{ }^{13} \mathbf{C} \mathbf{N M R}\left(150 \mathrm{MHz}, \mathrm{CDCl}_{3}\right) \delta 173.5$, $159.2,152.9,135.0,131.7,130.1,129.4,129.3,128.9,127.4,121.0,113.8,72.9,70.5,67.7,65.9,55.3$, 55.2, 52.5, 37.6, 33.9; HRMS (ES) calcd for $\mathrm{C}_{25} \mathrm{H}_{29} \mathrm{NO}_{6}[\mathrm{M}+\mathrm{Na}]$ 462.1887, found 462.1885.

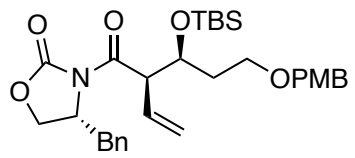

(R)-4-benzyl-3-((2R,3S)-3-((tert-butyldimethylsilyl)oxy)-5-((4methoxybenzyl)oxy)-2-vinylpentanoyl)oxazolidin-2-one (3). To a solution of S3 (17.56 g, $39.95 \mathrm{mmol}, 1 \mathrm{eq})$ and 2,6-lutidine (46.28 $\mathrm{mL}, 79.91 \mathrm{mmol}, 2 \mathrm{eq})$ in $\mathrm{CH}_{2} \mathrm{Cl}_{2}(135 \mathrm{~mL}, 0.3 \mathrm{M})$ was added tert-butylsilyltrifluoromethane sulfonate (11.6 $\mathrm{mL}, 59.9 \mathrm{mmol}, 1.5 \mathrm{eq})$ and stirred overnight. The mixture was quenched with $\mathrm{H}_{2} \mathrm{O}(100 \mathrm{~mL})$, stirred for $30 \mathrm{~min}$, and the aqueous layer was extracted with $\mathrm{CH}_{2} \mathrm{Cl}_{2}(2 \times 100 \mathrm{~mL})$. The combined organics were washed with $1 \mathrm{~N} \mathrm{HCl}$, sat. aq $\mathrm{NaHCO}_{3}$, brine, dried over $\mathrm{Na}_{2} \mathrm{SO}_{4}$, filtered and concentrated. The residue was purified by flash chromatography (20\% EtOAC/Hexanes) to give 3 (18.08 g, 84\% yield) as a pale yellow oil: $\mathrm{R}_{\mathrm{f}}=0.6(33 \% \mathrm{EtOAc} /$ Hexanes $) ;{ }^{1} \mathbf{H}$ NMR $\left(400 \mathrm{MHz}, \mathrm{CDCl}_{3}\right) \delta 7.33-7.26(\mathrm{~m}, 3 \mathrm{H}), 7.25(\mathrm{~d}, \mathrm{~J}$ $=8.8 \mathrm{~Hz}, 2 \mathrm{H}), 7.18(\mathrm{~d}, \mathrm{~J}=6.9 \mathrm{~Hz}, 2 \mathrm{H}), 6.85(\mathrm{~d}, \mathrm{~J}=8.6 \mathrm{~Hz}, 2 \mathrm{H}), 6.00$ (ddd, J = 9, 9.3, $18.2 \mathrm{~Hz}, 1 \mathrm{H}), 5.27$ $(\mathrm{d}, \mathrm{J}=10.2 \mathrm{~Hz}, 1 \mathrm{H}), 5.26(\mathrm{~d}, \mathrm{~J}=18.1 \mathrm{~Hz}, 1 \mathrm{H}), 4.58(\mathrm{dd}, \mathrm{J}=6.6 \mathrm{~Hz}, 8.8 \mathrm{~Hz}, 1 \mathrm{H}), 4.56-4.52(\mathrm{~m}, 1 \mathrm{H}), 4.41$ $(\mathrm{d}, \mathrm{J}=11.4 \mathrm{~Hz}, 1 \mathrm{H}), 4.36(\mathrm{~d}, \mathrm{~J}=11.5 \mathrm{~Hz}, 1 \mathrm{H}), 4.21(\mathrm{td}, \mathrm{J}=5.2 \mathrm{~Hz}, 6.4 \mathrm{~Hz}, 1 \mathrm{H}), 4.05(\mathrm{dd}, \mathrm{J}=2 \mathrm{~Hz}, 9.2 \mathrm{~Hz}$, 
$1 \mathrm{H}), 3.86(\mathrm{t}, \mathrm{J}=8.2 \mathrm{~Hz}, 1 \mathrm{H}), 3.78(\mathrm{~s}, 3 \mathrm{H}), 3.59(\mathrm{td}, \mathrm{J}=6.5,9.2 \mathrm{~Hz}, 1 \mathrm{H}), 3.48(\mathrm{dt}, \mathrm{J}=6.2,9.4 \mathrm{~Hz}, 1 \mathrm{H}), 3.23$ $(\mathrm{dd}, \mathrm{J}=3.0,13.4 \mathrm{~Hz}, 1 \mathrm{H}), 2.70(\mathrm{dd}, \mathrm{J}=9.7,13.4 \mathrm{~Hz}, 1 \mathrm{H}), 1.95-1.85(\mathrm{~m}, 2 \mathrm{H}), 0.86(\mathrm{~s}, 9 \mathrm{H}), 0.02(\mathrm{~s}, 3 \mathrm{H})$, $0.01(\mathrm{~s}, 3 \mathrm{H}) ;{ }^{13} \mathrm{C}$ NMR $\left(100 \mathrm{MHz}, \mathrm{CDCl}_{3}\right) \delta 172.6,159.2,153.0,135.6,134.2,130.9,129.7,129.5,129.1$, 127.5, 119.7, 113.9, 72.8, 71.3, 66.0, 65.9, 55.7, 55.5, 53.4, 37.7, 35.5, 26.0, 18.22, -4.2, -4.4; HRMS (ES) calcd for $\mathrm{C}_{31} \mathrm{H}_{43} \mathrm{NO}_{6} \mathrm{Si}[\mathrm{M}+\mathrm{Na}]$ 576.2752, found 576.2767.

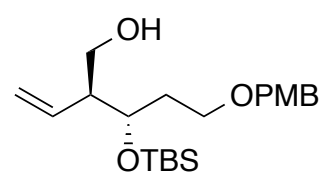

(2S,3S)-3-((tert-butyldimethylsilyl)oxy)-5-((4-methoxybenzyl)oxy)-2vinylpentan-1-ol (4). To a stirred solution of 3 (17.22 g, $31.14 \mathrm{mmol}, 1 \mathrm{eq})$ in THF $(625 \mathrm{~mL}, 0.05 \mathrm{M})$ at $0{ }^{\circ} \mathrm{C}$ was added a solution of $\mathrm{NaBH}_{4}(5.891 \mathrm{~g}, 155.7 \mathrm{mmol}, 5$ eq) in $\mathrm{H}_{2} \mathrm{O}(240 \mathrm{~mL})$. The mixture was stirred for $10 \mathrm{~min}$ at $0{ }^{\circ} \mathrm{C}$, then $5 \mathrm{~h}$ at r.t. The reaction was quenched by the addition of sat. aq $\mathrm{NH}_{4} \mathrm{Cl}$ solution $(200 \mathrm{~mL})$ and the mixture stirred at r.t. for $1 \mathrm{~h}$. The THF was removed under reduced pressure and the aqueous layer was extracted with EtOAc $(3 \mathrm{x}$ $75 \mathrm{~mL}$ ), the organics combined, washed with brine, dried over $\mathrm{Na}_{2} \mathrm{SO}_{4}$, filtered, and concentrated. The residue was purified by flash chromatography (18\% EtOAc/Hexanes) to afford 4 (10.04 g, 85\% yield) as a colorless oil; $\mathrm{R}_{\mathrm{f}}=0.25$ (20\% EtOAc/Hexanes); ${ }^{1} \mathbf{H}$ NMR $\left(400 \mathrm{MHz}, \mathrm{CDCl}_{3}\right) \delta 7.25(\mathrm{~d}, \mathrm{~J}=8.8 \mathrm{~Hz}, 2 \mathrm{H})$, $6.88(\mathrm{~d}, \mathrm{~J}=8.6 \mathrm{~Hz}, 2 \mathrm{H}), 5.71(\mathrm{ddd}, \mathrm{J}=17.3,10.4,8.6 \mathrm{~Hz}, 1 \mathrm{H}), 5.17(\mathrm{~d}, \mathrm{~J}=10.4 \mathrm{~Hz}, 1 \mathrm{H}), 5.10(\mathrm{~d}, \mathrm{~J}=17.4$ $\mathrm{Hz}, 1 \mathrm{H}), 4.43(\mathrm{~d}, \mathrm{~J}=11.5 \mathrm{~Hz}, 1 \mathrm{H}), 4.38(\mathrm{~d}, \mathrm{~J}=11.5 \mathrm{~Hz}, 1 \mathrm{H}), 4.04-3.94(\mathrm{~m}, 1 \mathrm{H}), 3.80$ (s, 3H), 3.75 (dt, J $=10.8,6.6 \mathrm{~Hz}, 1 \mathrm{H}), 3.61(\mathrm{ddd}, \mathrm{J}=11.0,7.0,5.0 \mathrm{~Hz}, 1 \mathrm{H}), 3.46(\mathrm{t}, \mathrm{J}=6.3 \mathrm{~Hz}, 2 \mathrm{H}), 2.43(\mathrm{qd}, \mathrm{J}=7.6,2.7 \mathrm{~Hz}$, 1H), $2.15(\mathrm{t}, \mathrm{J}=5.7 \mathrm{~Hz}, 1 \mathrm{H}), 1.88-1.68(\mathrm{~m}, 2 \mathrm{H}), 0.88(\mathrm{~s}, 9 \mathrm{H}), 0.09(\mathrm{~s}, 3 \mathrm{H}), 0.06(\mathrm{~s}, 3 \mathrm{H}) .{ }^{13} \mathbf{C}$ NMR $(100$ $\left.\mathrm{MHz}, \mathrm{CDCl}_{3}\right) \delta 159.3,135.6,130.6,129.4,118.7,113.9,72.7,71.1,66.6,63.7,55.4,51.3,34.1,26.0,18.1$, -4.4, -4.4; HRMS (ES) calcd for $\mathrm{C}_{21} \mathrm{H}_{36} \mathrm{O}_{4} \mathrm{Si}[\mathrm{M}+\mathrm{Na}]$ 403.2275, found 403.2271.

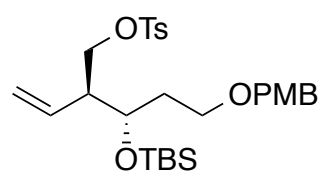

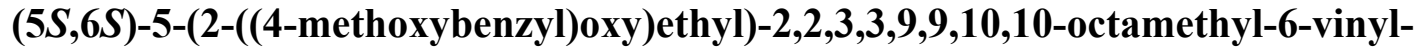
4,8-dioxa-3,9-disilaundecane (S4). ${ }^{4,5}$ To a stirred solution of 4 (9.81 g, $25.8 \mathrm{mmol}$, $1 \mathrm{eq})$ in dry pyridine $(250 \mathrm{~mL}, 0.1 \mathrm{M})$ at $0{ }^{\circ} \mathrm{C}$ was added $p$-toluenesulfonyl chloride (7.372 g, $38.66 \mathrm{mmol}, 1.5 \mathrm{eq}$ ). The mixture was stirred for $30 \mathrm{~min}$ at $0{ }^{\circ} \mathrm{C}$ and $4 \mathrm{~h}$ at rt. The mixture was re-cooling to $0{ }^{\circ} \mathrm{C}$ and was quenched by the slow addition of 0.5 $\mathrm{N} \mathrm{HCl}(300 \mathrm{~mL})$ and stirred at $0^{\circ} \mathrm{C}$ for $20 \mathrm{~min}$. The aqueous layer was extracted with diethyl ether $(3 \times 200$ $\mathrm{mL}$ ), organics combined, washed with $1 \mathrm{M} \mathrm{HCl}$, brine, dried over $\mathrm{Na}_{2} \mathrm{SO}_{4}$, filtered, and concentrated. The residue was purified using a chromatographic plug (18\% EtOAc/Hexanes) to afford $\mathbf{S 4}$ (11.67 g, 84\% yield) as a colorless oil: $\mathrm{R}_{\mathrm{f}}=0.4(20 \% \mathrm{EtOAc} /$ Hexanes $) ;{ }^{1} \mathbf{H}$ NMR $\left(400 \mathrm{MHz}, \mathrm{CDCl}_{3}\right) \delta 7.75(\mathrm{~d}, \mathrm{~J}=8.4 \mathrm{~Hz}, 2 \mathrm{H})$, $7.30(\mathrm{~d}, \mathrm{~J}=8.4 \mathrm{~Hz}, 2 \mathrm{H}), 7.21(\mathrm{~d}, \mathrm{~J}=8.4 \mathrm{~Hz}, 2 \mathrm{H}), 6.86(\mathrm{~d}, \mathrm{~J}=8.4 \mathrm{~Hz}, 2 \mathrm{H}), 5.58$ (ddd, J = 8.7, 10.3, 17.3 $\mathrm{Hz}, 1 \mathrm{H}), 5.12(\mathrm{dd}, \mathrm{J}=1.2,9.2 \mathrm{~Hz}, 1 \mathrm{H}), 5.04(\mathrm{~d}, \mathrm{~J}=17.2 \mathrm{~Hz}, 1 \mathrm{H}), 4.39(\mathrm{~d}, \mathrm{~J}=11.6 \mathrm{~Hz}, 1 \mathrm{H}), 4.33(\mathrm{~d}, \mathrm{~J}=$ $11.6 \mathrm{~Hz}, 1 \mathrm{H}), 4.08(\mathrm{dd}, \mathrm{J}=6.6,9.4 \mathrm{~Hz}, 1 \mathrm{H}), 3.94(\mathrm{dd}, \mathrm{J}=7.4,9.4 \mathrm{~Hz}, 1 \mathrm{H}), 3.93-3.89$ (m, 1H), 3.79 (s, $3 \mathrm{H}), 3.35(\mathrm{t}, \mathrm{J}=6.4 \mathrm{~Hz}, 2 \mathrm{H}), 2.46-2.42(\mathrm{~m}, 1 \mathrm{H}), 2.42(\mathrm{~s}, 3 \mathrm{H}), 1.74-1.57(\mathrm{~m}, 2 \mathrm{H}), 0.78(\mathrm{~s}, 9 \mathrm{H}),-0.01(\mathrm{~s}$, $3 \mathrm{H}),-0.05(\mathrm{~s}, 3 \mathrm{H}) ;{ }^{13} \mathbf{C}$ NMR $\left(100 \mathrm{MHz}, \mathrm{CDCl}_{3}\right) \delta 159.3,144.8,133.4,133.2,130.5,129.9,129.4,128.1$, $119.8,113.9,72.7,70.5,68.8,66.4,55.4,48.6,34.6,25.9,21.8,18.1,-4.3,-4.7$; HRMS (ES) calcd for $\mathrm{C}_{28} \mathrm{H}_{42} \mathrm{O}_{6} \mathrm{SSi}[\mathrm{M}+\mathrm{Na}]$ 557.2364, found 557.2370.

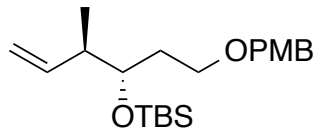

tert-butyl(((3S,4R)-1-((4-methoxybenzyl)oxy)-4-methylhex-5-en-3yl)oxy)dimethylsilane (5). ${ }^{6}$ To a stirred solution of $\mathbf{S 4}(3.72 \mathrm{~g}, 6.96 \mathrm{mmol}, 1 \mathrm{eq})$ in dry THF $(70 \mathrm{~mL}, 0.1 \mathrm{M})$ at $0{ }^{\circ} \mathrm{C}$ was added lithium triethylborohydride $(1 \mathrm{M}$ in $\mathrm{THF}$, $17.39 \mathrm{~mL}, 17.39 \mathrm{mmol}, 2.5 \mathrm{eq})$ over $10 \mathrm{~min}$. The reaction was stirred at $0{ }^{\circ} \mathrm{C}$, warmed to rt and stirred for an additional $2 \mathrm{~h}$. The mixture was cooled to $0{ }^{\circ} \mathrm{C}$, quenched with the slow addition of water $(15 \mathrm{~mL}), 3 \mathrm{~N}$ $\mathrm{NaOH}(15 \mathrm{~mL}), 30 \% \mathrm{H}_{2} \mathrm{O}_{2}(15 \mathrm{~mL})$, and stirred for an additional $30 \mathrm{~min}$. The organics were removed under reduced pressure and the aqueous layer was extracted with EtOAc $(3 \times 50 \mathrm{~mL})$. The combined organics were washed with brine, dried over $\mathrm{Na}_{2} \mathrm{SO}_{4}$, filtered, and concentrated. The residue was purified by flash chromatography (10\% EtOAc/Hexanes) to afford $\mathbf{5}$ ( $1.87 \mathrm{~g}, 74 \%$ yield) as a clear and colorless oil: $\mathrm{R}_{\mathrm{f}}=0.5$ 
(10\% EtOAc/Hexanes); ${ }^{1} \mathbf{H}$ NMR $\left(600 \mathrm{MHz}, \mathrm{CDCl}_{3}\right) \delta 7.25(\mathrm{~d}, \mathrm{~J}=8.3 \mathrm{~Hz}, 2 \mathrm{H}), 6.87(\mathrm{~d}, \mathrm{~J}=8.2 \mathrm{~Hz}, 2 \mathrm{H})$, $5.76(\mathrm{ddd}, \mathrm{J}=17.4,10.1,7.3 \mathrm{~Hz}, 1 \mathrm{H}), 4.99(\mathrm{~d}, \mathrm{~J}=9.8 \mathrm{~Hz}, 1 \mathrm{H}), 4.98(\mathrm{~d}, \mathrm{~J}=18.1 \mathrm{~Hz}, 1 \mathrm{H}), 4.43(\mathrm{~d}, \mathrm{~J}=11.5$ $\mathrm{Hz}, 1 \mathrm{H}), 4.38(\mathrm{~d}, \mathrm{~J}=11.5 \mathrm{~Hz}, 1 \mathrm{H}), 3.80(\mathrm{~s}, 3 \mathrm{H}), 3.76(\mathrm{dt}, \mathrm{J}=8.0,4.2 \mathrm{~Hz}, 1 \mathrm{H}), 3.52-3.42(\mathrm{~m}, 2 \mathrm{H}), 2.29$ (td, J = 7.1, 3.9 Hz, 1H), $1.74-1.60(\mathrm{~m}, 2 \mathrm{H}), 0.99$ (d, J = 6.9 Hz, 3H), 0.88 (s, 9H), 0.05 (s, 3H), 0.03 (s, $3 \mathrm{H}) .{ }^{13} \mathrm{C}$ NMR $\left(100 \mathrm{MHz}, \mathrm{CDCl}_{3}\right) \delta 159.2,140.8,130.9,129.4,114.7,113.9,72.7,72.6,67.2,55.4,43.6$, 33.4, 26.1, 18.3, 14.7, -4.3, -4.4; HRMS (ES) calcd for $\mathrm{C}_{21} \mathrm{H}_{36} \mathrm{O}_{3} \mathrm{Si}[\mathrm{M}+\mathrm{H}]$ 364.2434, found 364.2439.

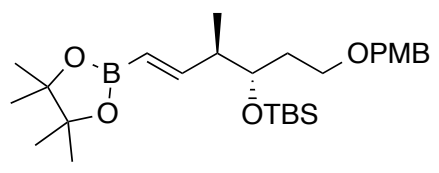

(tert-butyl $(((3 S, 4 R, E)-1-((4-m e t h o x y b e n z y l) o x y)-4-m e t h y l-6-(4,4,5,5-$ tetramethyl-1,3,2-dioxaborolan-2-yl)hex-5-en-3-yl)oxy)dimethylsilane (S6). ${ }^{7}$ To a solution of Hoveyda Grubbs II $(0.10 \mathrm{~g}, 0.16 \mathrm{mmol}, 0.05 \mathrm{eq})$ in dry, degassed $\mathrm{CH}_{2} \mathrm{Cl}_{2}(15 \mathrm{~mL}, 0.2 \mathrm{M})$ ) was added olefin 5 (1.15 g, $3.15 \mathrm{mmol}, 1$ eq), followed by vinylboronic acid pinacol ester $\left(1.07 \mathrm{~mL}, 6.31 \mathrm{mmol}, 3 \mathrm{eq}\right.$, passed through a plug of $\mathrm{SiO}_{2}$ using $10 \% \mathrm{EtOAc} / \mathrm{Hex}$ as the eluent to remove stabilizer immediately prior to use) and the reaction was heated at reflux for $18 \mathrm{~h}$. The reaction was cooled, concentrated, and purified directly by flash chromatography (5\% EtOAc/Hexanes) to afford $\mathbf{S 6}\left(1.10 \mathrm{~g}, 74 \%\right.$ yield) as a pale yellow oil: $\mathrm{R}_{\mathrm{f}}=0.35(5 \%$ EtOAc/Hexanes); ${ }^{1} \mathbf{H}$ NMR $\left(400 \mathrm{MHz}, \mathrm{CDCl}_{3}\right) \delta 7.25(\mathrm{~d}, \mathrm{~J}=8.1 \mathrm{~Hz}, 2 \mathrm{H}), 6.87(\mathrm{~d}, \mathrm{~J}=8.0 \mathrm{~Hz}, 2 \mathrm{H}), 6.56$ $(\mathrm{dd}, \mathrm{J}=18.1,6.6 \mathrm{~Hz}, 1 \mathrm{H}), 5.43(\mathrm{~d}, \mathrm{~J}=18.1 \mathrm{~Hz}, 1 \mathrm{H}), 4.41(\mathrm{~d}, \mathrm{~J}=18.0 \mathrm{~Hz}, 1 \mathrm{H}), 4.38(\mathrm{~d}, \mathrm{~J}=17.9 \mathrm{~Hz}, 1 \mathrm{H})$, $3.87-3.75(\mathrm{~m}, 1 \mathrm{H}), 3.80(\mathrm{~d}, \mathrm{~J}=0.8 \mathrm{~Hz}, 3 \mathrm{H}), 3.55-3.38(\mathrm{~m}, 2 \mathrm{H}), 2.40(\mathrm{q}, \mathrm{J}=6.2 \mathrm{~Hz}, 1 \mathrm{H}), 1.66(\mathrm{q}, \mathrm{J}=6.7$ $\mathrm{Hz}, 2 \mathrm{H}), 1.26(\mathrm{~s}, 12 \mathrm{H}), 1.00(\mathrm{~d}, \mathrm{~J}=6.9 \mathrm{~Hz}, 3 \mathrm{H}), 0.87(\mathrm{~d}, \mathrm{~J}=0.8 \mathrm{~Hz}, 9 \mathrm{H}), 0.03(\mathrm{~s}, 3 \mathrm{H}), 0.02(\mathrm{~s}, 3 \mathrm{H}) ;{ }^{13} \mathrm{C}$ NMR $\left(100 \mathrm{MHz}, \mathrm{CDCl}_{3}\right) \delta 159.3,156.2,130.9,129.5,114.0,83.3,72.8,72.5,67.4,55.5,45.5,32.9,26.1$, 25.1, 25.0, 18.3, 13.3, -4.2, -4.4; HRMS (ES) calcd for $\mathrm{C}_{27} \mathrm{H}_{47} \mathrm{BO}_{5} \mathrm{Si}[\mathrm{M}+\mathrm{H}]$ 491.3359, found 491.3350.

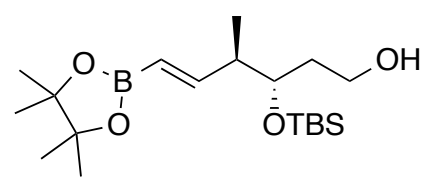

(3S,4R,E)-3-((tert-butyldimethylsilyl)oxy)-4-methyl-6-(4,4,5,5-tetramethyl1,3,2-dioxaborolan-2-yl)hex-5-en-1-ol (S7). To a solution of S6 (1.90 g, 3.87 mmol, $1 \mathrm{eq})$ in $\mathrm{CH}_{2} \mathrm{Cl}_{2}(25 \mathrm{~mL}, 0.15 \mathrm{M})$ and water $(1.49 \mathrm{~mL})$ at r.t. was added DDQ (1.32 g, $5.81 \mathrm{mmol}, 1.5 \mathrm{eq})$ and the mixture was stirred for $1 \mathrm{~h}$. The reaction was quenched with sat. $\mathrm{NaHCO}_{3}$, stirred for 10 minutes, and diluted with water. The aqueous layer was extracted with $\mathrm{CH}_{2} \mathrm{Cl}_{2}(3 \times 50 \mathrm{~mL})$, the organics were combined, dried over $\mathrm{Na}_{2} \mathrm{SO}_{4}$, filtered, and concentrated. The residue was purified by flash chromatography $(12 \%$ EtOAc/Hexanes) to afford $\mathbf{S} 7(1.21 \mathrm{~g}, 83 \%$ yield $)$ as a colorless oil: $\mathrm{R}_{\mathrm{f}}=0.15(10 \%$ EtOAc/Hexanes $) ;{ }^{1} \mathbf{H}$ NMR $\left(600 \mathrm{MHz}, \mathrm{CDCl}_{3}\right) \delta 6.54(\mathrm{dd}, \mathrm{J}=18.1,6.6 \mathrm{~Hz}, 1 \mathrm{H}), 5.46(\mathrm{dd}, \mathrm{J}=18.1,1.5 \mathrm{~Hz}, 1 \mathrm{H}), 3.87(\mathrm{ddd}, \mathrm{J}=$ 7.9, 5.0, 3.8 Hz, 1H), $3.77-3.67(\mathrm{~m}, 2 \mathrm{H}), 2.49(\mathrm{pdd}, \mathrm{J}=6.8,5.0,1.5 \mathrm{~Hz}, 1 \mathrm{H}), 2.03(\mathrm{t}, \mathrm{J}=5.4 \mathrm{~Hz}, 1 \mathrm{H}), 1.71$ $-1.63(\mathrm{~m}, 2 \mathrm{H}), 1.26(\mathrm{~d}, \mathrm{~J}=1.7 \mathrm{~Hz}, 12 \mathrm{H}), 1.01(\mathrm{~d}, \mathrm{~J}=6.8 \mathrm{~Hz}, 3 \mathrm{H}), 0.89(\mathrm{~s}, 9 \mathrm{H}), 0.08(\mathrm{~s}, 3 \mathrm{H}), 0.07$ (s, $3 \mathrm{H}) ;{ }^{13} \mathrm{C}$ NMR $\left(150 \mathrm{MHz}, \mathrm{CDCl}_{3}\right) \delta 155.7,83.07,74.2,60.6,44.9,34.1,25.8,24.8,24.7,17.9,12.8,-4.4$, -4.70; HRMS (ES) calcd for $\mathrm{C}_{19} \mathrm{H}_{39} \mathrm{BO}_{4} \mathrm{Si}[\mathrm{M}+\mathrm{H}] 371.2783$, found 371.2778.

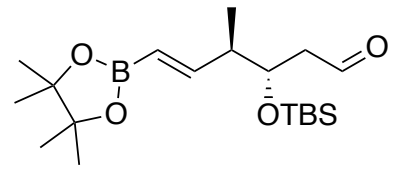

$(3 S, 4 R, E)-3-(($ tert-butyldimethylsilyl)oxy)-4-methyl-6-(4,4,5,5-tetramethyl1,3,2-dioxaborolan-2-yl)hex-5-enal (6). To an open round bottom flask was added alcohol $\mathbf{S 7}(1.00 \mathrm{~g}, 4.09 \mathrm{mmol}, 1 \mathrm{eq})$ in $\mathrm{CH}_{2} \mathrm{Cl}_{2}(41 \mathrm{~mL}, 0.1 \mathrm{M})$ and treated with $\mathrm{NaHCO}_{3}(1.72 \mathrm{~g}, 20.45 \mathrm{mmol}, 5 \mathrm{eq})$ and desmartin periodinane (2.08 g, 4.91

mmol, $1.2 \mathrm{eq})$. The reaction was stirred at $\mathrm{rt}$ for $1 \mathrm{hr}$, quenched with $10 \%$ aq. $\mathrm{Na}_{2} \mathrm{~S}_{2} \mathrm{O}_{3}$ solution $(50 \mathrm{~mL})$, and stirred until both layers were clear. The organics were separated, and the aqueous layer extracted with $\mathrm{CH}_{2} \mathrm{Cl}_{2}(2 \times 50 \mathrm{~mL})$. The combined organics were washed with brine, dried over sodium sulfate, filtered, and concentrated. The residue was purified by flash chromatography (10\% EtOAc/Hexanes) to afford 6 (0.768 g, 77\% yield) as a colorless oil: $\mathrm{R}_{\mathrm{f}}=0.5$ (10\% EtOAc/Hexanes); ${ }^{1} \mathbf{H} \mathbf{N M R}\left(400 \mathrm{MHz}, \mathrm{CDCl}_{3}\right) \delta 9.75$ $(\mathrm{dd}, \mathrm{J}=2.1,1.1 \mathrm{~Hz}, 1 \mathrm{H}), 6.50(\mathrm{dd}, \mathrm{J}=18.1,6.6 \mathrm{~Hz}, 1 \mathrm{H}), 5.46(\mathrm{~d}, \mathrm{~J}=18.0 \mathrm{~Hz}, 1 \mathrm{H}), 4.22$ (dt, J = 8.1, 4.2 $\mathrm{Hz}, 1 \mathrm{H}), 2.54-2.42(\mathrm{~m}, 2 \mathrm{H}), 2.41-2.33(\mathrm{~m}, 1 \mathrm{H}), 1.25$ (s, 12H), 1.01 (dd, J = 6.9, $0.9 \mathrm{~Hz}, 3 \mathrm{H}), 0.85$ (d, J $=0.8 \mathrm{~Hz}, 9 \mathrm{H}), 0.05(\mathrm{~s}, 3 \mathrm{H}), 0.02(\mathrm{~s}, 3 \mathrm{H}) ;{ }^{13} \mathbf{C ~ N M R}\left(100 \mathrm{MHz}, \mathrm{CDCl}_{3}\right) \delta 202.5,154.7,83.4,70.9,47.2$, 
45.5, 29.9, 25.9, 25.0, 24.9, 18.2, 13.0, -4.3, -4.6; HRMS (ES) calcd for $\mathrm{C}_{19} \mathrm{H}_{37} \mathrm{BO}_{4} \mathrm{Si}[\mathrm{M}+\mathrm{H}]$ 368.2554, found 368.2550

Unit B Synthetic Experimentals

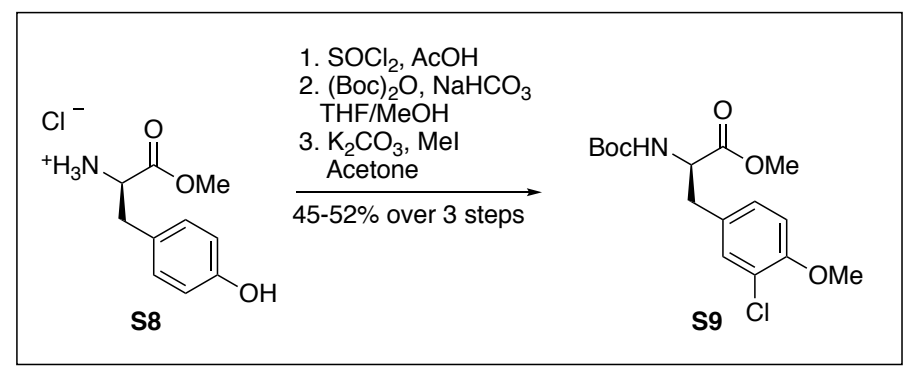

S9 was synthesized in three steps from commercially available $\mathbf{S 8}$ as previously reported. ${ }^{8}$

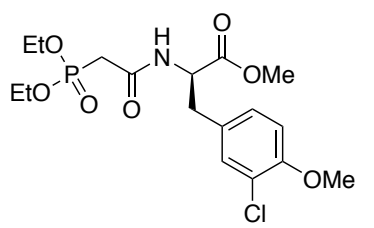

methyl(R)-3-(3-chloro-4-methoxyphenyl)-2-(2-(diethoxyphosphoryl) acetamido)propanoate (7). To an open flask was added $\mathbf{S 9}$ (1.10 g, $3.20 \mathrm{mmol}, 1$ eq) and $4 \mathrm{M} \mathrm{HCl} /$ Dioxane $(20 \mathrm{~mL})$. The mixture was vigorously stirred for $30 \mathrm{~min}$ and concentrated under reduced pressure. The resulting white solid was used directly.

Deprotected S9 was suspended in DMF $(32 \mathrm{~mL}, 0.1 \mathrm{M})$ and treated with 2(diethoxyphosphoryl)acetic acid (0.692 g, $3.52 \mathrm{mmol}, 1.1 \mathrm{eq}), \mathrm{EDC} \cdot \mathrm{HCl}(0.731 \mathrm{~g}, 3.84 \mathrm{mmol}, 1.2 \mathrm{eq})$, HOBt hydrate $(0.591 \mathrm{~g}, 3.84 \mathrm{mmol}, 1.2 \mathrm{eq})$, and DIPEA (1.03 g, $1.40 \mathrm{~mL}, 8.00 \mathrm{mmol}, 2.5 \mathrm{eq})$ and stirred at $\mathrm{rt}$ for $18 \mathrm{~h}$. The reaction was quenched with half sat. aq $\mathrm{NH}_{4} \mathrm{Cl}(30 \mathrm{~mL})$, the aqueous layer extracted $(3 \mathrm{x}$ $30 \mathrm{~mL}$ ) with DCM, organics combined, dried over sodium sulfate, filtered, and concentrated. The residue was purified by flash chromatography system (1-7\% MeOH/DCM) to afford $7(0.90 \mathrm{mg}, 67 \%$ yield $)$ as a clear and colorless oil: $\mathrm{R}_{\mathrm{f}}=0.2(2.5 \% \mathrm{MeOH} / \mathrm{DCM}) ;{ }^{1} \mathbf{H} \mathbf{N M R}\left(600 \mathrm{MHz}, \mathrm{CDCl}_{3}\right) \delta 7.16(\mathrm{~d}, \mathrm{~J}=2.2 \mathrm{~Hz}$, 1H), 7.09 (bd, J = 7.5 Hz, 1H), $7.04(\mathrm{dd}, \mathrm{J}=8.4,2.2 \mathrm{~Hz}, 1 \mathrm{H}), 6.84(\mathrm{~d}, \mathrm{~J}=8.4 \mathrm{~Hz}, 1 \mathrm{H}), 4.78(\mathrm{td}, \mathrm{J}=7.1,5.4$ $\mathrm{Hz}, 1 \mathrm{H}), 4.13(\mathrm{dt}, \mathrm{J}=14.9,7.2 \mathrm{~Hz}, 1 \mathrm{H}), 4.06(\mathrm{dq}, \mathrm{J}=8.2,7.1 \mathrm{~Hz}, 2 \mathrm{H}), 3.85(\mathrm{~s}, 3 \mathrm{H}), 3.70(\mathrm{~s}, 3 \mathrm{H}), 3.08$ (dd, $\mathrm{J}=14.2,5.4 \mathrm{~Hz}, 1 \mathrm{H}), 2.97(\mathrm{dd}, \mathrm{J}=14.2,6.8 \mathrm{~Hz}, 1 \mathrm{H}), 2.85(\mathrm{~d}, \mathrm{~J}=10.7 \mathrm{~Hz}, 1 \mathrm{H}), 2.81(\mathrm{~d}, \mathrm{~J}=10.5 \mathrm{~Hz}, 1 \mathrm{H})$, $1.31(\mathrm{t}, \mathrm{J}=7.1 \mathrm{~Hz}, 3 \mathrm{H}), 1.27(\mathrm{t}, \mathrm{J}=7.1 \mathrm{~Hz}, 3 \mathrm{H}) ;{ }^{13} \mathbf{C} \mathbf{N M R}\left(150 \mathrm{MHz}, \mathrm{CDCl}_{3}\right) \delta 171.5,163.9,154.2,131.1$, 129.1, 128.7, 122.5, 112.2, 62.9, 56.2, 53.9, 36.9, 35.7, 34.8, 16.5; HRMS (ES) calcd for $\mathrm{C}_{17} \mathrm{H}_{25} \mathrm{ClNO}_{7} \mathrm{P}$ $[\mathrm{M}+\mathrm{H}] \mathrm{421.1057}$, found 421.1053 .

\section{Unit A/B Analogue Synthetic Experimentals}

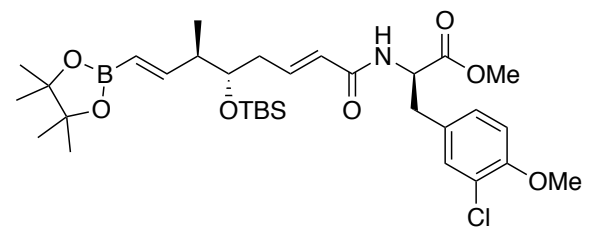

methyl $(R)-2-((2 E, 5 S, 6 R, 7 E)-5-((t e r t-b u t y l d i m e t h y l s i l y l) o x y)-6-$ methyl-8-(4,4,5,5-tetramethyl-1,3,2-dioxaborolan-2-yl)octa-2,7dienamido)-3-(3-chloro-4-methoxyphenyl)propanoate (8). A suspension of $7(0.63 \mathrm{~g}, 1.49 \mathrm{mmol}, 1 \mathrm{eq})$ in dry THF $(15 \mathrm{~mL}, 0.1 \mathrm{M})$ was cooled to $0{ }^{\circ} \mathrm{C}$ and treated with $\mathrm{NaH}(60 \%$ suspension in oil, 0.055

$\mathrm{g}, 1.64 \mathrm{mmol}, 1.1 \mathrm{eq})$. The reaction was stirred for $30 \mathrm{~min}$ prior to the dropwise addition of aldehyde 6 $(0.55 \mathrm{~g}, 1.49 \mathrm{mmol}, 1 \mathrm{eq})$ in THF $(5 \mathrm{~mL})$. The reaction was allowed to stir at $0{ }^{\circ} \mathrm{C}$ for $1 \mathrm{~h}$ and quenched with half sat. $\mathrm{NH}_{4} \mathrm{Cl}(10 \mathrm{~mL})$. The organics were removed under reduced pressure, the remaining aqueous layer was extracted with DCM $(3 \times 50 \mathrm{~mL})$, organics combined, washed with brine, dried over sodium sulfate, filtered, and concentrated. The crude product was purified by flash chromatography system $(6-45 \%$ EtOAc/Hexanes) to afford $8(0.545 \mathrm{~g}, 57 \%$ yield $)$ as a clear and colorless oil: $\mathrm{R}_{\mathrm{f}}=0.25(25 \%$ EtOAc/Hexanes); ${ }^{1} \mathbf{H}$ NMR : $\left(400 \mathrm{MHz}, \mathrm{CD}_{3} \mathrm{OD}\right) \delta 7.21(\mathrm{~d}, \mathrm{~J}=2.2 \mathrm{~Hz}, 1 \mathrm{H}), 7.10(\mathrm{dd}, \mathrm{J}=8.5,2.2 \mathrm{~Hz}, 1 \mathrm{H})$, $6.97(\mathrm{~d}, \mathrm{~J}=8.4 \mathrm{~Hz}, 1 \mathrm{H}), 6.75(\mathrm{dt}, \mathrm{J}=15.2,7.5 \mathrm{~Hz}, 1 \mathrm{H}), 6.57(\mathrm{dd}, \mathrm{J}=18.0,7.7 \mathrm{~Hz}, 1 \mathrm{H}), 5.96(\mathrm{~d}, \mathrm{~J}=15.3$ 
$\mathrm{Hz}, 1 \mathrm{H}), 5.38(\mathrm{~d}, \mathrm{~J}=18.3 \mathrm{~Hz}, 1 \mathrm{H}), 4.68(\mathrm{dd}, \mathrm{J}=8.9,5.5 \mathrm{~Hz}, 1 \mathrm{H}), 3.84(\mathrm{~s}, 3 \mathrm{H}), 3.73(\mathrm{q}, \mathrm{J}=5.4 \mathrm{~Hz}, 1 \mathrm{H})$, $3.70(\mathrm{~s}, 3 \mathrm{H}), 3.11(\mathrm{dd}, \mathrm{J}=14.0,5.6 \mathrm{~Hz}, 1 \mathrm{H}), 2.90(\mathrm{dd}, \mathrm{J}=14.0,9.0 \mathrm{~Hz}, 1 \mathrm{H}), 2.41-2.25(\mathrm{~m}, 3 \mathrm{H}), 1.26(\mathrm{~s}$, $12 \mathrm{H}), 1.01(\mathrm{~d}, \mathrm{~J}=6.8 \mathrm{~Hz}, 3 \mathrm{H}), 0.89(\mathrm{~s}, 9 \mathrm{H}), 0.06(\mathrm{~s}, 3 \mathrm{H}), 0.03(\mathrm{~s}, 3 \mathrm{H}) ;{ }^{13} \mathbf{C}$ NMR: $\left(100 \mathrm{MHz}, \mathrm{CD}_{3} \mathrm{OD}\right) \delta$ $173.3,168.0,157.3,155.4,143.3,131.8,131.3,129.7,126.3,123.2,113.3,84.4,76.1,56.5,55.2,52.7$, 46.5, 38.4, 37.3, 26.5, 25.1, 25.1, 19.0, 15.8, -4.0, -4.3; HRMS (ESI) calcd for $\mathrm{C}_{32} \mathrm{H}_{51} \mathrm{BCINO}_{7} \mathrm{Si}[\mathrm{M}+\mathrm{H}]$ 635.3216, found 635.3215 .

General Suzuki coupling procedure: To a long tube was added 8 ( 1 eq), $\mathrm{K}_{3} \mathrm{PO}_{4}(2.5 \mathrm{eq})$, aryl iodide (2 eq), and $\operatorname{Pd}_{2}(\mathrm{dba})_{3}(0.05 \mathrm{eq})$ and suspended in a mixture 1,2 dichloroethane and water $(4: 1,0.1 \mathrm{M})$ and stirred vigorously until completion as assessed by TLC ( $2-12$ hours). The reaction was diluted with $0.5 \mathrm{M}$ $\mathrm{HCl}$ and EtOAc, and the aqueous layer was extracted with EtOAc (3 x $20 \mathrm{~mL})$. The organics were combined, dried over $\mathrm{Na}_{2} \mathrm{SO}_{4}$, filtered, and purified by flash chromatography system as indicated below.

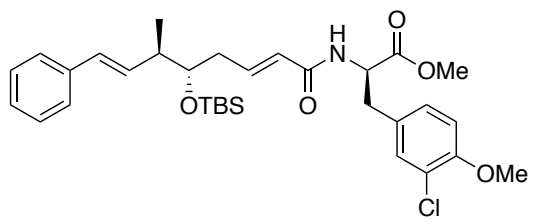

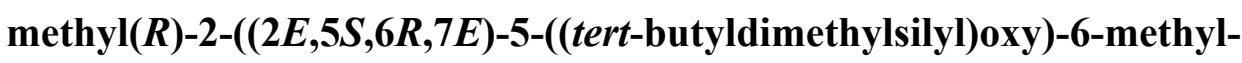
8-phenylocta-2,7-dienamido)-3-(3-chloro-4methoxyphenyl)propanoate (9a). Reaction was run as per general Suzuki procedure, and purified by flash chromatography system $\left(\mathrm{SiO}_{2}, 20-50 \%\right.$ EtOAc/Hexanes) to afford $9 \mathrm{a}\left(0.086 \mathrm{~g}, 93 \%\right.$ yield) as a pale yellow oil: $\mathrm{R}_{\mathrm{f}}$ $=0.35$ (25\% EtOAc/Hexanes); ${ }^{1} \mathbf{H}$ NMR (400 MHz, CD $\left.{ }_{3} \mathrm{OD}\right) \delta 7.33(\mathrm{~d}, \mathrm{~J}=7.5 \mathrm{~Hz}, 2 \mathrm{H}), 7.27(\mathrm{t}, \mathrm{J}=7.6$ $\mathrm{Hz}, 2 \mathrm{H}), 7.21(\mathrm{~d}, \mathrm{~J}=2.2 \mathrm{~Hz}, 1 \mathrm{H}), 7.18(\mathrm{t}, \mathrm{J}=7.2 \mathrm{~Hz}, 1 \mathrm{H}), 7.10(\mathrm{dd}, \mathrm{J}=8.4,2.2 \mathrm{~Hz}, 1 \mathrm{H}), 6.96(\mathrm{~d}, \mathrm{~J}=8.4$ $\mathrm{Hz}, 1 \mathrm{H}), 6.78(\mathrm{dt}, \mathrm{J}=15.2,7.5 \mathrm{~Hz}, 1 \mathrm{H}), 6.38(\mathrm{~d}, \mathrm{~J}=16.0 \mathrm{~Hz}, 1 \mathrm{H}), 6.19(\mathrm{dd}, \mathrm{J}=16.0,8.2 \mathrm{~Hz}, 1 \mathrm{H}), 5.96(\mathrm{~d}$, $\mathrm{J}=15.4 \mathrm{~Hz}, 1 \mathrm{H}), 4.69$ (dd, $\mathrm{J}=8.9,5.6 \mathrm{~Hz}, 1 \mathrm{H}), 3.83$ (s, 3H), 3.80 (q, 1H), 3.70 (s, 3H), 3.11 (dd, J = 14.0, $5.6 \mathrm{~Hz}, 1 \mathrm{H}), 2.90(\mathrm{dd}, \mathrm{J}=14.0,9.0 \mathrm{~Hz}, 1 \mathrm{H}), 2.44(\mathrm{q}, \mathrm{J}=6.5 \mathrm{~Hz}, 1 \mathrm{H}), 2.37(\mathrm{t}, \mathrm{J}=6.9 \mathrm{~Hz}, 2 \mathrm{H}), 1.11(\mathrm{~d}, \mathrm{~J}=$ $6.9 \mathrm{~Hz}, 3 \mathrm{H}), 0.90$ (s, 9H), 0.06 (s, 3H), 0.05 (s, 3H); $\left.{ }^{13} \mathbf{C ~ N M R ~ ( 1 0 0 ~ M H z , ~ C D ~}{ }_{3} \mathrm{OD}\right) 173.3,168.0,155.4$, $143.5,139.0,133.1,131.8,131.7,131.3,129.7,129.5,128.0,127.1,126.2,123.2,113.3,76.5,56.5,55.2$, 52.7, 44.1, 38.6, 37.3, 26.4, 19.0, 16.8, -4.0, -4.3.; HRMS (ES) calcd for $\mathrm{C}_{32} \mathrm{H}_{44} \mathrm{ClNO}_{5} \mathrm{Si}[\mathrm{M}+\mathrm{H}]$ 586.2750, found 586.2754.

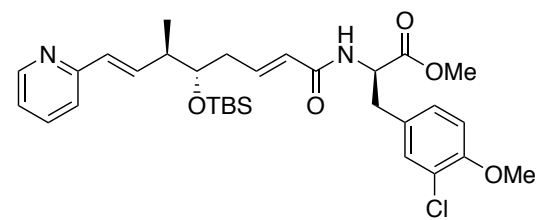

methyl $(R)-2-((2 E, 5 S, 6 R, 7 E)-5-((t e r t-b u t y l d i m e t h y l s i l y l) o x y)-6-$ methyl-8-(pyridin-2-yl)octa-2,7-dienamido)-3-(3-chloro-4methoxyphenyl)propanoate (9b). Reaction was run as per general Suzuki procedure, and purified by flash chromatography $\left(\mathrm{Si}-\mathrm{NH}_{2}, 20-\right.$ $50 \%$ EtOAc/Hexanes) to afford $9 \mathrm{~b}(0.078 \mathrm{~g}, 45 \%$ yield $)$ as a pale yellow oil: $\mathrm{R}_{\mathrm{f}}=0.15(50 \%$ EtOAc/Hexanes $){ }^{1} \mathbf{H}$ NMR (400 MHz, $\left.\mathrm{CD}_{3} \mathrm{OD}\right) \delta 8.44$ $(\mathrm{d}, \mathrm{J}=4.0 \mathrm{~Hz}, 1 \mathrm{H}), 7.76(\mathrm{td}, \mathrm{J}=7.8,1.8 \mathrm{~Hz}, 1 \mathrm{H}), 7.46(\mathrm{~d}, \mathrm{~J}=8.0 \mathrm{~Hz}, 1 \mathrm{H}), 7.24(\mathrm{dd}, \mathrm{J}=7.8,5.2 \mathrm{~Hz}, 1 \mathrm{H})$, $7.21(\mathrm{~d}, \mathrm{~J}=2.1 \mathrm{~Hz}, 1 \mathrm{H}), 7.10(\mathrm{dd}, \mathrm{J}=8.5,2.2 \mathrm{~Hz}, 1 \mathrm{H}), 6.96(\mathrm{~d}, \mathrm{~J}=8.3 \mathrm{~Hz}, 1 \mathrm{H}), 6.77(\mathrm{dt}, \mathrm{J}=15.3,7.4 \mathrm{~Hz}$, $1 \mathrm{H}), 6.64(\mathrm{dd}, \mathrm{J}=16.0,8.2 \mathrm{~Hz}, 1 \mathrm{H}), 6.49(\mathrm{~d}, \mathrm{~J}=16.0 \mathrm{~Hz}, 1 \mathrm{H}), 5.98(\mathrm{~d}, \mathrm{~J}=15.4 \mathrm{~Hz}, 1 \mathrm{H}), 4.70(\mathrm{dd}, \mathrm{J}=9.0$, $5.5 \mathrm{~Hz}, 1 \mathrm{H}), 3.87-3.84(\mathrm{~m}, 1 \mathrm{H}), 3.83(\mathrm{~m}, 3 \mathrm{H}), 3.70(\mathrm{~s}, 3 \mathrm{H}), 3.11(\mathrm{dd}, \mathrm{J}=14.0,5.5 \mathrm{~Hz}, 1 \mathrm{H}), 2.90(\mathrm{dd}, \mathrm{J}=$ 14.0, $9.0 \mathrm{~Hz}, 1 \mathrm{H}), 2.51(\mathrm{~h}, \mathrm{~J}=6.8 \mathrm{~Hz}, 1 \mathrm{H}), 2.38(\mathrm{t}, \mathrm{J}=6.8 \mathrm{~Hz}, 2 \mathrm{H}), 1.14(\mathrm{~d}, \mathrm{~J}=6.8 \mathrm{~Hz}, 3 \mathrm{H}), 0.90$ (s, 9H), 0.06 (s, 3H), 0.05 (s, 3H). ${ }^{13}$ C NMR (100 MHz, CD 3 OD) $\delta 173.27,167.9,157.2,155.4,149.7,143.2,139.0$, 138.7, 131.8, 131.3, 131.1, 129.7, 126.4, 123.4, 123.2, 122.3, 113.3, 76.3, 56.5, 55.2, 52.7, 44.1, 38.6, 37.3, 26.4, 19.0, 16.5, -4.0, -4.3. HRMS (ESI) calcd for $\mathrm{C}_{31} \mathrm{H}_{43} \mathrm{ClN}_{2} \mathrm{O}_{5} \mathrm{Si}[\mathrm{M}+\mathrm{H}]$ 587.2703, found 587.2705.

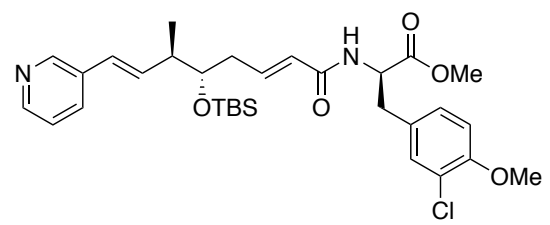

methyl $(R)-2-((2 E, 5 S, 6 R, 7 E)-5-((t e r t-b u t y l d i m e t h y l s i l y l) o x y)-6-$ methyl-8-(pyridin-3-yl)octa-2,7-dienamido)-3-(3-chloro-4methoxyphenyl)propanoate (9c). Reaction was run as per general Suzuki procedure, and purified by flash chromatography system $\left(\mathrm{Si}-\mathrm{NH}_{2}\right.$, $16-60 \%$ EtOAc/Hexanes) to afford 9c $(0.049 \mathrm{~g}, 72 \%$ yield $)$ as a pale yellow oil: $\mathrm{R}_{\mathrm{f}}=0.15$ (50\% EtOAc/Hexanes); ${ }^{1} \mathbf{H}$ NMR $\left(600 \mathrm{MHz}, \mathrm{CD}_{3} \mathrm{OD}\right) \delta 8.49(\mathrm{~d}, \mathrm{~J}=2.1 \mathrm{~Hz}, 1 \mathrm{H}), 8.35$ $(\mathrm{dd}, \mathrm{J}=4.9,1.5 \mathrm{~Hz}, 1 \mathrm{H}), 7.85(\mathrm{~d}, \mathrm{~J}=8.0 \mathrm{~Hz}, 1 \mathrm{H}), 7.37(\mathrm{dd}, \mathrm{J}=8.0,4.8 \mathrm{~Hz}, 1 \mathrm{H}), 7.21(\mathrm{~d}, \mathrm{~J}=2.1 \mathrm{~Hz}, 1 \mathrm{H})$, 
$7.10(\mathrm{dd}, \mathrm{J}=8.4,2.2 \mathrm{~Hz}, 1 \mathrm{H}), 6.96(\mathrm{~d}, \mathrm{~J}=8.5 \mathrm{~Hz}, 1 \mathrm{H}), 6.78(\mathrm{dt}, \mathrm{J}=15.2,7.5 \mathrm{~Hz}, 1 \mathrm{H}), 6.43(\mathrm{~d}, \mathrm{~J}=16.1 \mathrm{~Hz}$, $1 \mathrm{H}), 6.36(\mathrm{dd}, \mathrm{J}=16.1,7.9 \mathrm{~Hz}, 1 \mathrm{H}), 5.97(\mathrm{dt}, \mathrm{J}=15.3,1.4 \mathrm{~Hz}, 1 \mathrm{H}), 4.70(\mathrm{dd}, \mathrm{J}=8.9,5.6 \mathrm{~Hz}, 1 \mathrm{H}), 3.85-$ $3.79(\mathrm{~m}, 1 \mathrm{H}), 3.83(\mathrm{~s}, 3 \mathrm{H}), 3.70(\mathrm{~s}, 3 \mathrm{H}), 3.11(\mathrm{dd}, \mathrm{J}=14.0,5.6 \mathrm{~Hz}, 1 \mathrm{H}), 2.91(\mathrm{dd}, \mathrm{J}=14.0,9.0 \mathrm{~Hz}, 1 \mathrm{H})$, $2.49(\mathrm{td}, \mathrm{J}=7.2,4.7 \mathrm{~Hz}, 1 \mathrm{H}), 2.38(\mathrm{ddd}, \mathrm{J}=7.4,5.8,1.4 \mathrm{~Hz}, 2 \mathrm{H}), 1.13(\mathrm{~d}, \mathrm{~J}=6.9 \mathrm{~Hz}, 3 \mathrm{H}), 0.90(\mathrm{~s}, 9 \mathrm{H})$, $0.06(\mathrm{~s}, 3 \mathrm{H}), 0.05(\mathrm{~s}, 3 \mathrm{H}) ;{ }^{13} \mathbf{C}$ NMR $\left(150 \mathrm{MHz}, \mathrm{CD}_{3} \mathrm{OD}\right) \delta 173.3,168.0,155.4,148.3,148.1,143.2,136.9$, $135.5,134.7,131.8,131.3,129.7,127.7,126.3,125.3,123.2,113.4,76.3,56.6,55.1,52.7,44.24,38.7$, 37.3, 26.4, 19.0, 16.6, -4.0, -4.3; HRMS (ESI) calcd for $\mathrm{C}_{31} \mathrm{H}_{43} \mathrm{ClN}_{2} \mathrm{O}_{5} \mathrm{Si}[\mathrm{M}+\mathrm{H}]$ 587.2703, found 587.2702.

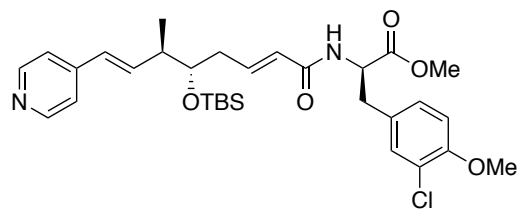

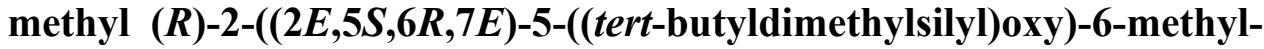
8-(pyridin-4-yl)octa-2,7-dienamido)-3-(3-chloro-4methoxyphenyl)propanoate (9d). Reaction was run as per general Suzuki procedure, and purified by flash chromatography system $\left(\mathrm{Si}-\mathrm{NH}_{2}, 15-55 \%\right.$ EtOAc/Hexanes $)$ to afford $9 d(0.062 \mathrm{~g}, 71 \%$ yield $)$ as a pale yellow oil: $\mathrm{R}_{\mathrm{f}}$ $=0.25\left(50 \%\right.$ EtOAc/Hexanes); ${ }^{1} \mathbf{H}$ NMR $\left(600 \mathrm{MHz}, \mathrm{CD}_{3} \mathrm{OD}\right) \delta \delta 8.42(\mathrm{~d}, \mathrm{~J}=5.9 \mathrm{~Hz}, 2 \mathrm{H}), 7.37(\mathrm{~d}, \mathrm{~J}=6.3$ $\mathrm{Hz}, 2 \mathrm{H}), 7.21(\mathrm{~d}, \mathrm{~J}=2.1 \mathrm{~Hz}, 1 \mathrm{H}), 7.10(\mathrm{dd}, \mathrm{J}=8.5,2.1 \mathrm{~Hz}, 1 \mathrm{H}), 6.97(\mathrm{~d}, \mathrm{~J}=8.4 \mathrm{~Hz}, 1 \mathrm{H}), 6.78(\mathrm{dt}, \mathrm{J}=15.2$, $7.5 \mathrm{~Hz}, 1 \mathrm{H}), 6.56(\mathrm{dd}, \mathrm{J}=16.0,8.2 \mathrm{~Hz}, 1 \mathrm{H}), 6.41(\mathrm{~d}, \mathrm{~J}=16.0 \mathrm{~Hz}, 1 \mathrm{H}), 5.97(\mathrm{~d}, \mathrm{~J}=15.3 \mathrm{~Hz}, 1 \mathrm{H}), 4.69$ (dd, $\mathrm{J}=9.0,5.5 \mathrm{~Hz}, 1 \mathrm{H}), 3.86-3.81(\mathrm{~m}, 1 \mathrm{H}), 3.83(\mathrm{~s}, 3 \mathrm{H}), 3.70(\mathrm{~s}, 3 \mathrm{H}), 3.11(\mathrm{dd}, \mathrm{J}=14.1,5.6 \mathrm{~Hz}, 1 \mathrm{H}), 2.91$ $(\mathrm{dd}, \mathrm{J}=14.1,9.0 \mathrm{~Hz}, 1 \mathrm{H}), 2.51(\mathrm{td}, \mathrm{J}=13.3,6.5 \mathrm{~Hz}, 1 \mathrm{H}), 2.38(\mathrm{t}, \mathrm{J}=6.9 \mathrm{~Hz}, 2 \mathrm{H}), 1.13(\mathrm{~d}, \mathrm{~J}=6.8 \mathrm{~Hz}, 3 \mathrm{H})$, $0.90(\mathrm{~s}, 9 \mathrm{H}), 0.06(\mathrm{~s}, 3 \mathrm{H}), 0.06(\mathrm{~s}, 3 \mathrm{H}) .{ }^{13} \mathbf{C} \mathbf{N M R}\left(150 \mathrm{MHz}, \mathrm{CD}_{3} \mathrm{OD}\right){ }^{13} \mathrm{C}$ NMR $\left(150 \mathrm{MHz}, \mathrm{CD}_{3} \mathrm{OD}\right) \delta$ $173.3,168.0,155.4,150.2,147.7,143.1,140.0,131.8,131.4,129.7,129.2,126.4,123.2,122.3,113.4,76.2$, $56.6,55.1,52.7,44.2,38.7,37.3,26.4,19.0,16.6,-4.0,-4.4$; HRMS (ESI) calcd for $\mathrm{C}_{31} \mathrm{H}_{43} \mathrm{ClN}_{2} \mathrm{O}_{5} \mathrm{Si}[\mathrm{M}+\mathrm{H}]$ 587.2703 , found 587.2699.

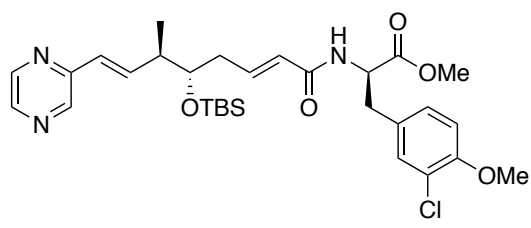

methyl

(R)-2-((2E,5S,6R,7E)-5-((tert-butyldimethylsilyl)oxy)-6methyl-8-(pyrazin-2-yl)octa-2,7-dienamido)-3-(3-chloro-4methoxyphenyl)propanoate (9e). Reaction was run as per general Suzuki procedure, and purified by flash chromatography system $\left(\mathrm{Si}-\mathrm{NH}_{2}\right.$, $20-55 \%$ EtOAc/Hexanes) to afford 9e $(0.115 \mathrm{~g}, 65 \%$ yield $)$ as a pale yellow oil: $\mathrm{R}_{\mathrm{f}}=0.2\left(50 \%\right.$ EtOAc/Hexanes); ${ }^{1} \mathbf{H}$ NMR $\left(600 \mathrm{MHz}, \mathrm{CD}_{3} \mathrm{OD}\right) \delta 8.57(\mathrm{~d}, \mathrm{~J}=1.5 \mathrm{~Hz}, 1 \mathrm{H}), 8.51$ $(\mathrm{s}, 1 \mathrm{H}), 8.39(\mathrm{~d}, \mathrm{~J}=2.6 \mathrm{~Hz}, 1 \mathrm{H}), 7.21(\mathrm{~d}, \mathrm{~J}=2.2 \mathrm{~Hz}, 1 \mathrm{H}), 7.10(\mathrm{dd}, \mathrm{J}=8.4,2.2 \mathrm{~Hz}, 1 \mathrm{H}), 6.96(\mathrm{~d}, \mathrm{~J}=8.5$ $\mathrm{Hz}, 1 \mathrm{H}), 6.90(\mathrm{dd}, \mathrm{J}=15.9,8.3 \mathrm{~Hz}, 1 \mathrm{H}), 6.78(\mathrm{dt}, \mathrm{J}=15.2,7.5 \mathrm{~Hz}, 1 \mathrm{H}), 6.54(\mathrm{~d}, \mathrm{~J}=16.0 \mathrm{~Hz}, 1 \mathrm{H}), 5.98(\mathrm{~d}$, $\mathrm{J}=15.4 \mathrm{~Hz}, 1 \mathrm{H}), 4.70(\mathrm{dd}, \mathrm{J}=8.9,5.5 \mathrm{~Hz}, 1 \mathrm{H}), 3.86-3.84(\mathrm{~m}, 1 \mathrm{H}), 3.83(\mathrm{~s}, 3 \mathrm{H}), 3.70(\mathrm{~s}, 3 \mathrm{H}), 3.11(\mathrm{dd}, \mathrm{J}$ $=14.0,5.6 \mathrm{~Hz}, 1 \mathrm{H}), 2.91(\mathrm{dd}, \mathrm{J}=14.0,9.0 \mathrm{~Hz}, 1 \mathrm{H}), 2.55(\mathrm{q}, \mathrm{J}=6.9 \mathrm{~Hz}, 1 \mathrm{H}), 2.41-2.33(\mathrm{~m}, 2 \mathrm{H}), 1.15(\mathrm{~d}$, $\mathrm{J}=6.8 \mathrm{~Hz}, 3 \mathrm{H}), 0.89(\mathrm{~s}, 9 \mathrm{H}), 0.06(\mathrm{~s}, 3 \mathrm{H}), 0.05(\mathrm{~s}, 3 \mathrm{H}) ;{ }^{13} \mathrm{C}$ NMR $\left(150 \mathrm{MHz}, \mathrm{CD}_{3} \mathrm{OD}\right) \delta 173.3,167.9$, 155.4, 153.0, 145.5, 143.9, 143.5, 143.1, 141.8, 131.8, 131.3, 129.7, 127.9, 126.4, 123.19, 113.4, 76.2, 56.6, 55.5, 52.7, 44.1, 38.7, 37.3, 26.4, 19.0, 16.5, -4.0, -4.4; HRMS (ESI) calcd for $\mathrm{C}_{30} \mathrm{H}_{42} \mathrm{ClN}_{3} \mathrm{O}_{5} \mathrm{Si}[\mathrm{M}+\mathrm{H}]$ 588.2655 , found 588.2659.

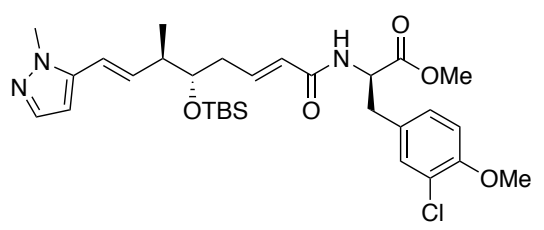

methyl $\quad(R)-2-((2 E, 5 S, 6 R, 7 E)-5-((t e r t-b u t y l d i m e t h y l s i l y l) o x y)-6-$ methyl-8-(1-methyl-1H-pyrazol-5-yl)octa-2,7-dienamido)-3-(3chloro-4-methoxyphenyl)propanoate (9f). Reaction was run as per general Suzuki procedure, and purified by flash chromatography system $\left(\mathrm{Si}-\mathrm{NH}_{2}, 20-70 \% \mathrm{EtOAc} / \mathrm{Hexanes}\right)$ to afford $9 \mathrm{f}(0.044 \mathrm{~g}, 63 \%$ yield $)$ as a pale yellow oil: $\mathrm{R}_{\mathrm{f}}=0.1$ (50\% EtOAc/Hexanes); ${ }^{1} \mathbf{H}$ NMR $\left(600 \mathrm{MHz}, \mathrm{CD}_{3} \mathrm{OD}\right) \delta 7.34(\mathrm{~d}, \mathrm{~J}=2.0 \mathrm{~Hz}, 1 \mathrm{H})$, $7.20(\mathrm{~d}, \mathrm{~J}=2.2 \mathrm{~Hz}, 1 \mathrm{H}), 7.10(\mathrm{dd}, \mathrm{J}=8.4,2.2 \mathrm{~Hz}, 1 \mathrm{H}), 6.96(\mathrm{~d}, \mathrm{~J}=8.4 \mathrm{~Hz}, 1 \mathrm{H}), 6.78(\mathrm{dt}, \mathrm{J}=15.2,7.5 \mathrm{~Hz}$, $1 \mathrm{H}), 6.38(\mathrm{~d}, \mathrm{~J}=15.9 \mathrm{~Hz}, 1 \mathrm{H}), 6.33(\mathrm{~d}, \mathrm{~J}=2.1 \mathrm{~Hz}, 1 \mathrm{H}), 6.21(\mathrm{dd}, \mathrm{J}=15.9,8.3 \mathrm{~Hz}, 1 \mathrm{H}), 5.97(\mathrm{dt}, \mathrm{J}=15.4$, $1.3 \mathrm{~Hz}, 1 \mathrm{H}), 4.69$ (dd, J = 8.9, $5.6 \mathrm{~Hz}, 1 \mathrm{H}), 3.82(\mathrm{~s}, 3 \mathrm{H}), 3.81-3.79(\mathrm{~m}, 1 \mathrm{H}), 3.80(\mathrm{~s}, 3 \mathrm{H}), 3.70(\mathrm{~s}, 3 \mathrm{H}), 3.11$ $(\mathrm{dd}, \mathrm{J}=14.0,5.6 \mathrm{~Hz}, 1 \mathrm{H}), 2.91(\mathrm{dd}, \mathrm{J}=14.0,8.9 \mathrm{~Hz}, 1 \mathrm{H}), 2.48(\mathrm{~h}, \mathrm{~J}=6.9 \mathrm{~Hz}, 1 \mathrm{H}), 2.38(\mathrm{t}, \mathrm{J}=6.5 \mathrm{~Hz}, 2 \mathrm{H})$, $1.12(\mathrm{~d}, \mathrm{~J}=6.9 \mathrm{~Hz}, 3 \mathrm{H}), 0.89(\mathrm{~s}, 9 \mathrm{H}), 0.06(\mathrm{~s}, 3 \mathrm{H}), 0.05(\mathrm{~s}, 3 \mathrm{H}) .{ }^{13} \mathbf{C}$ NMR $\left(150 \mathrm{MHz}, \mathrm{CD}_{3} \mathrm{OD}\right) \delta 173.3$, 
168.0, 155.4, 143.1, 142.8, 139.1, 138.5, 131.8, 131.4, 129.7, 126.3, 123.2, 118.1, 113.4, 103.4, 76.2, 56.6, $55.2,52.7,44.2,38.8,37.3,36.4,26.4,19.0,16.8,-4.0,-4.4$; HRMS (ESI) calcd for $\mathrm{C}_{30} \mathrm{H}_{44} \mathrm{ClN}_{3} \mathrm{O}_{5} \mathrm{Si}$ [M+H] 590.2812 , found 590.2811 .

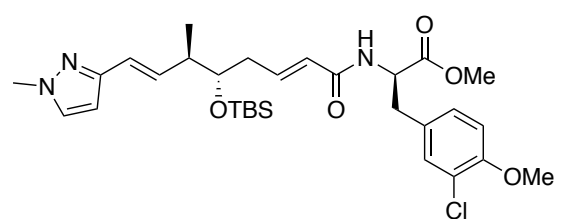

methyl (R)-2-((2E,5S,6R,7E)-5-((tert-butyldimethylsilyl)oxy)-6methyl-8-(1-methyl-1H-pyrazol-3-yl)octa-2,7-dienamido)-3-(3chloro-4-methoxyphenyl)propanoate $(9 \mathrm{~g})$. Reaction was run as per general Suzuki procedure, and purified by flash chromatography (Si$\mathrm{NH}_{2}, 16$ - 55\% EtOAc/Hexanes) to afford $9 \mathrm{~g}(0.065 \mathrm{~g}, 71 \%$ yield $)$ as a pale yellow oil: $\mathrm{R}_{\mathrm{f}}=0.25$ (50\% EtOAc/Hexanes); ${ }^{1} \mathbf{H}$ NMR $\left(600 \mathrm{MHz}, \mathrm{CD}_{3} \mathrm{OD}\right) \delta 7.46(\mathrm{~d}, \mathrm{~J}=2.3 \mathrm{~Hz}, 1 \mathrm{H})$, $7.21(\mathrm{~d}, \mathrm{~J}=2.2 \mathrm{~Hz}, 1 \mathrm{H}), 7.10(\mathrm{dd}, \mathrm{J}=8.5,2.2 \mathrm{~Hz}, 1 \mathrm{H}), 6.96(\mathrm{~d}, \mathrm{~J}=8.4 \mathrm{~Hz}, 1 \mathrm{H}), 6.75(\mathrm{dt}, \mathrm{J}=15.2,7.5 \mathrm{~Hz}$, $1 \mathrm{H}), 6.30(\mathrm{~d}, \mathrm{~J}=2.3 \mathrm{~Hz}, 1 \mathrm{H}), 6.17(\mathrm{dd}, \mathrm{J}=16.2,8.1 \mathrm{~Hz}, 1 \mathrm{H}), 5.96(\mathrm{~d}, \mathrm{~J}=15.4 \mathrm{~Hz}, 1 \mathrm{H}), 4.69(\mathrm{dd}, \mathrm{J}=9.0$, $5.6 \mathrm{~Hz}, 1 \mathrm{H}), 3.84(\mathrm{~s}, 3 \mathrm{H}), 3.83(\mathrm{~s}, 3 \mathrm{H}), 3.78(\mathrm{q}, \mathrm{J}=5.6 \mathrm{~Hz}, 1 \mathrm{H}), 3.70(\mathrm{~s}, 3 \mathrm{H}), 3.11$ (dd, J = 14.1, $5.6 \mathrm{~Hz}$, $1 \mathrm{H}), 2.91(\mathrm{dd}, \mathrm{J}=14.0,9.0 \mathrm{~Hz}, 1 \mathrm{H}), 2.42(\mathrm{p}, \mathrm{J}=6.7 \mathrm{~Hz}, 1 \mathrm{H}), 2.38-2.30(\mathrm{~m}, 2 \mathrm{H}), 1.09(\mathrm{~d}, \mathrm{~J}=6.8 \mathrm{~Hz}, 3 \mathrm{H})$, 0.90 (s, 9H), 0.06 (s, 3H), 0.04 (s, 3H); ${ }^{13} \mathbf{C}$ NMR (150 MHz, CD $\left.{ }_{3} \mathrm{OD}\right) \delta$ 173.3, 168.0, 155.4, 152.1, 143.4, 135.0, 133.0, 131.8, 131.4, 129.7, 126.3, 123.3, 123.2, 113.4, 103.2, 76.4, 56.5, 55.2, 52.7, 43.9, 38.6, 38.5, 37.3, 26.5, 19.0, 16.5, -4.0, -4.3; HRMS (ESI) calcd for $\mathrm{C}_{30} \mathrm{H}_{44} \mathrm{ClN}_{3} \mathrm{O}_{5} \mathrm{Si}[\mathrm{M}+\mathrm{H}]$ 590.2812, found 590.2809.

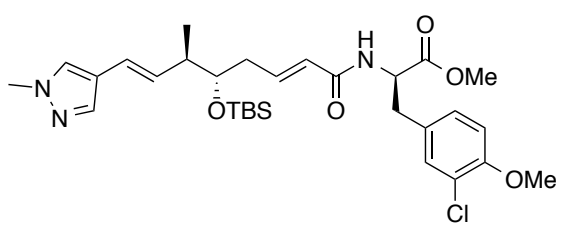

methyl $\quad(R)-2-((2 E, 5 S, 6 R, 7 E)-5-((t e r t-b u t y l d i m e t h y l s i l y l) o x y)-6-$ methyl-8-(1-methyl-1H-pyrazol-4-yl)octa-2,7-dienamido)-3-(3chloro-4-methoxyphenyl)propanoate (9h). Reaction was run as per general Suzuki procedure, and purified by flash chromatography system (Si-NH $2,16-60 \%$ EtOAc/Hexanes) to afford 9 h $(0.085 \mathrm{~g}, 92 \%$ yield $)$ as a pale yellow oil: $\mathrm{R}_{\mathrm{f}}=0.2\left(50 \%\right.$ EtOAc/Hexanes); ${ }^{1} \mathbf{H}$ NMR $\left(400 \mathrm{MHz}, \mathrm{CD}_{3} \mathrm{OD}\right) \delta 7.53(\mathrm{~s}, 1 \mathrm{H}), 7.47$ (s, $1 \mathrm{H}), 7.21(\mathrm{~d}, \mathrm{~J}=2.2 \mathrm{~Hz}, 1 \mathrm{H}), 7.10(\mathrm{dd}, \mathrm{J}=8.4,2.2 \mathrm{~Hz}, 1 \mathrm{H}), 6.96(\mathrm{~d}, \mathrm{~J}=8.4 \mathrm{~Hz}, 1 \mathrm{H}), 6.77(\mathrm{dt}, \mathrm{J}=15.2,7.5$ $\mathrm{Hz}, 1 \mathrm{H}), 6.18(\mathrm{~d}, \mathrm{~J}=16.0 \mathrm{~Hz}, 1 \mathrm{H}), 5.95(\mathrm{~d}, \mathrm{~J}=15.4 \mathrm{~Hz}, 1 \mathrm{H}), 5.89$ (dd, J = 16.1, 8.2 Hz, 1H), 4.69 (dd, J = 8.9, $5.6 \mathrm{~Hz}, 1 \mathrm{H}), 3.84(\mathrm{~s}, 3 \mathrm{H}), 3.83(\mathrm{~s}, 3 \mathrm{H}), 3.75(\mathrm{q}, \mathrm{J}=5.5 \mathrm{~Hz}, 1 \mathrm{H}), 3.70(\mathrm{~s}, 3 \mathrm{H}), 3.11(\mathrm{dd}, \mathrm{J}=14.0,5.6$ $\mathrm{Hz}, 1 \mathrm{H}), 2.90(\mathrm{dd}, \mathrm{J}=14.0,9.0 \mathrm{~Hz}, 1 \mathrm{H}), 2.41-2.27(\mathrm{~m}, 3 \mathrm{H}), 1.06(\mathrm{~d}, \mathrm{~J}=6.8 \mathrm{~Hz}, 3 \mathrm{H}), 0.89$ (s, $9 \mathrm{H}), 0.05$ $(\mathrm{s}, 3 \mathrm{H}), 0.03(\mathrm{~s}, 3 \mathrm{H}) .{ }^{13} \mathbf{C}$ NMR $\left(150 \mathrm{MHz}, \mathrm{CD}_{3} \mathrm{OD}\right) \delta 173.3,168.0,155.4,143.7,137.6,131.8,131.6,131.3$, $129.7,129.3,126.1,123.2,122.4,121.2,113.36,76.60,56.55,55.15,52.72,44.16,38.72,38.32,37.28$, 26.44, 18.98, 16.45, -4.05, -4.31; HRMS (ESI) calcd for $\mathrm{C}_{30} \mathrm{H}_{44} \mathrm{ClN}_{3} \mathrm{O}_{5} \mathrm{Si}[\mathrm{M}+\mathrm{H}]$ 590.2812, found 590.2813 .

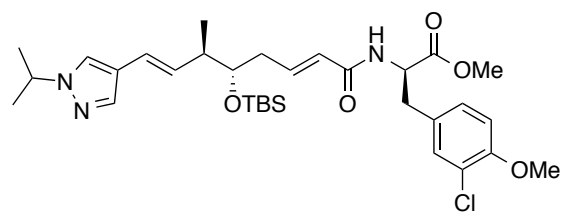

methyl $(R)-2-((2 E, 5 S, 6 R, 7 E)-5-((t e r t-b u t y l d i m e t h y l s i l y l) 0 x y)-8-(1-$ isopropyl-1H-pyrazol-4-yl)-6-methylocta-2,7-dienamido)-3-(3chloro-4-methoxyphenyl)propanoate (9i). Reaction was run as per general Suzuki procedure, and purified by flash chromatography (Amine column, 20 - 50\% EtOAc/Hexanes) to afford 9i $(0.056 \mathrm{~g}, 83 \%$ yield) as a pale yellow oil: $\mathrm{R}_{\mathrm{f}}=0.35$ (50\% EtOAc/Hexanes); ${ }^{1} \mathbf{H}$ NMR $\left(600 \mathrm{MHz}, \mathrm{CD}_{3} \mathrm{OD}\right) \delta{ }^{1} \mathrm{H}$ NMR $(400 \mathrm{MHz}, \text { Methanol-d })_{4} \delta 7.61(\mathrm{~s}, 1 \mathrm{H}), 7.48(\mathrm{~s}, 1 \mathrm{H}), 7.21(\mathrm{~d}, \mathrm{~J}=2.2 \mathrm{~Hz}, 1 \mathrm{H}), 7.10(\mathrm{dd}, \mathrm{J}=8.4,2.2 \mathrm{~Hz}$, $1 \mathrm{H}), 6.96(\mathrm{~d}, \mathrm{~J}=8.4 \mathrm{~Hz}, 1 \mathrm{H}), 6.77(\mathrm{dt}, \mathrm{J}=15.2,7.5 \mathrm{~Hz}, 1 \mathrm{H}), 6.20(\mathrm{~d}, \mathrm{~J}=16.1 \mathrm{~Hz}, 1 \mathrm{H}), 5.95(\mathrm{~d}, \mathrm{~J}=15.3$ $\mathrm{Hz}, 1 \mathrm{H}), 5.90(\mathrm{dd}, \mathrm{J}=16.1,8.1 \mathrm{~Hz}, 1 \mathrm{H}), 4.69$ (dd, J = 8.9, $5.6 \mathrm{~Hz}, 1 \mathrm{H}), 4.46$ (hept, J = 7.0 Hz, 1H), 3.83 (s, $3 \mathrm{H}), 3.75$ (q, J = 5.4 Hz, 1H), $3.70(\mathrm{~s}, 3 \mathrm{H}), 3.11(\mathrm{dd}, \mathrm{J}=14.0,5.6 \mathrm{~Hz}, 1 \mathrm{H}), 2.91(\mathrm{dd}, \mathrm{J}=14.0,8.9 \mathrm{~Hz}, 1 \mathrm{H})$, $2.46-2.29(\mathrm{~m}, 3 \mathrm{H}), 1.46(\mathrm{~d}, \mathrm{~J}=6.7 \mathrm{~Hz}, 6 \mathrm{H}), 1.07(\mathrm{~d}, \mathrm{~J}=6.9 \mathrm{~Hz}, 3 \mathrm{H}), 0.90(\mathrm{~s}, 9 \mathrm{H}), 0.06(\mathrm{~s}, 3 \mathrm{H}), 0.03(\mathrm{~s}$, $3 \mathrm{H}) .{ }^{13} \mathrm{C}$ NMR $\left(150 \mathrm{MHz}, \mathrm{CD}_{3} \mathrm{OD}\right) \delta{ }^{13} \mathrm{C}$ NMR (101 MHz, CD $\left.3 \mathrm{OD}\right) \delta 173.3,168.0,155.4,143.7,137.2$, $131.8,131.3,129.7,126.1,123.2$, 121.9, 121.4, 113.4, 76.6, 56.5, 55.2, 54.0, 52.7, 44.2, 38.3, 37.3, 26.4, 23.1, 19.0, 16.4, -4.0, -4.3; HRMS (ESI) calcd for $\mathrm{C}_{32} \mathrm{H}_{48} \mathrm{ClN}_{3} \mathrm{O}_{5} \mathrm{Si}[\mathrm{M}+\mathrm{H}]$ 618.3125, found 618.3129. 


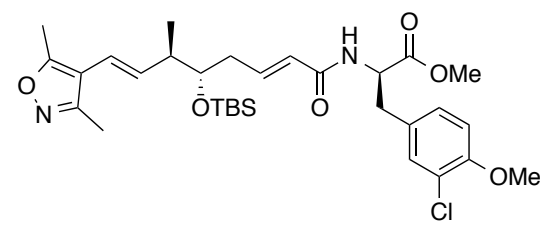

methyl $(R)-2-((2 E, 5 S, 6 R, 7 E)-5-((t e r t-b u t y l d i m e t h y l s i l y l) 0 x y)-8-(3,5-$ dimethylisoxazol-4-yl)-6-methylocta-2,7-dienamido)-3-(3-chloro-4methoxyphenyl)propanoate (9j). Reaction was run as per general Suzuki procedure, and purified by flash chromatography (Amine column, $10-$ $50 \%$ EtOAc/Hexanes) to afford $9 \mathbf{j}(0.057 \mathrm{~g}, 85 \%$ yield $)$ as a pale yellow oil: $\mathrm{R}_{\mathrm{f}}=0.35$ (33\% EtOAc/Hexanes); ${ }^{\mathbf{1}} \mathbf{H}$ NMR: $\left(400 \mathrm{MHz}, \mathrm{CD}_{3} \mathrm{OD}\right) \delta 7.20(\mathrm{~d}, \mathrm{~J}=2.2 \mathrm{~Hz}, 1 \mathrm{H}), 7.10$ (dd, $\mathrm{J}=8.3,2.2 \mathrm{~Hz}, 1 \mathrm{H}), 6.96(\mathrm{~d}, \mathrm{~J}=8.4 \mathrm{~Hz}, 1 \mathrm{H}), 6.76(\mathrm{dt}, \mathrm{J}=15.2,7.6 \mathrm{~Hz}, 1 \mathrm{H}), 6.11(\mathrm{~d}, \mathrm{~J}=16.3 \mathrm{~Hz}, 1 \mathrm{H}), 5.96$ (d, J = 15.4 Hz, 1H), $5.90(\mathrm{dd}, \mathrm{J}=16.4,8.5 \mathrm{~Hz}, 1 \mathrm{H}), 4.68(\mathrm{dd}, \mathrm{J}=9.0,5.5 \mathrm{~Hz}, 1 \mathrm{H}), 3.84(\mathrm{~s}, 3 \mathrm{H}), 3.83-$ $3.77(\mathrm{~m}, 1 \mathrm{H}), 3.70(\mathrm{~s}, 3 \mathrm{H}), 3.12(\mathrm{dd}, \mathrm{J}=14.0,5.5 \mathrm{~Hz}, 1 \mathrm{H}), 2.90(\mathrm{dd}, \mathrm{J}=14.0,9.0 \mathrm{~Hz}, 1 \mathrm{H}), 2.46-2.33(\mathrm{~m}$, $3 \mathrm{H}), 2.38(\mathrm{~s}, 3 \mathrm{H}) 2.26(\mathrm{~s}, 3 \mathrm{H}), 1.11(\mathrm{~d}, \mathrm{~J}=6.9 \mathrm{~Hz}, 3 \mathrm{H}), 0.90(\mathrm{~s}, 9 \mathrm{H}), 0.07(\mathrm{~s}, 3 \mathrm{H}), 0.06(\mathrm{~s}, 3 \mathrm{H}) .{ }^{13} \mathrm{C}$ NMR: $\left(100 \mathrm{MHz}, \mathrm{CD}_{3} \mathrm{OD}\right) \delta 173.3,168.0,166.5,159.6,155.4,143.2,135.8,131.8,131.3,129.7,126.2,123.2$, $119.0,114.2,113.4,76.3,56.5,55.2,52.7,44.65,39.2,37.3,26.4,19.0,17.7,11.5,11.4,-3.9,-4.3$; HRMS (ESI) calcd for $\mathrm{C}_{31} \mathrm{H}_{45} \mathrm{ClN}_{2} \mathrm{O}_{6} \mathrm{Si}[\mathrm{M}+\mathrm{H}]$ 605.2808, found 605.2801.

\section{Unit C/D Synthetic Experimentals}

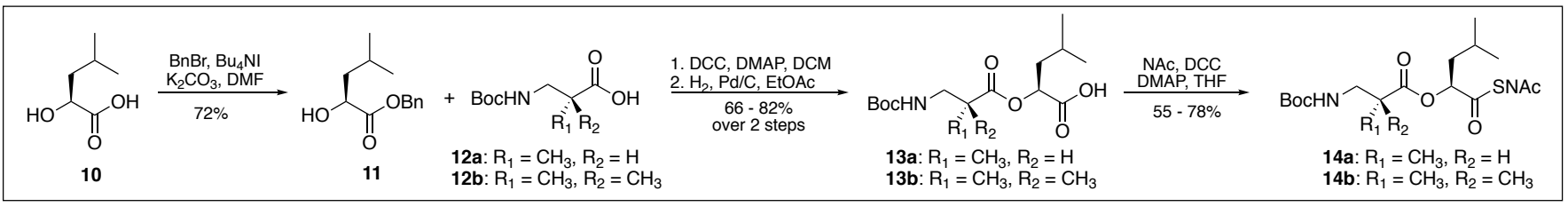

13a and 13b were synthesized from commercially available 10, all spectra were in accordance with published literature. ${ }^{9-12}$

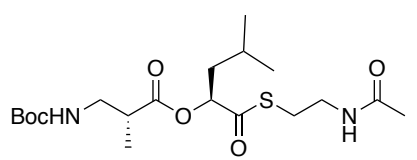

(S)-1-((2-acetamidoethyl)thio)-4-methyl-1-oxopentan-2-yl (R)-3-((tertbutoxycarbonyl)amino)-2-methylpropanoate (14a). To a solution of 13a in DMF (0.1M) was added $\mathrm{EDC} \mathrm{HCl}(1.5 \mathrm{eq})$, and $\mathrm{HOBt}(1.2 \mathrm{eq})$, and the reaction was stirred for $30 \mathrm{~min}$. NAc was added and stirred for $10 \mathrm{~min}$ prior to the

addition of DMAP ( $0.05 \mathrm{eq})$. The reaction was stirred for 12 hours, diluted with water and EtOAc and the aqueous layer was extracted $(2 \times 40 \mathrm{~mL})$, organics combined, washed with sat. $\mathrm{NH}_{4} \mathrm{Cl}(2 \times 100 \mathrm{~mL})$, and dried over sodium sulfate. The organics were removed under reduced pressure and the remaining residue was purified by flash chromatography system $(1-10 \% \mathrm{MeOH} / \mathrm{DCM})$ to afford 21 as white solid. Spectra was in accord with published literature. ${ }^{13}$<smiles>CCCC(C)C(=O)OCC(C)CSCCNC(C)=O</smiles>

(S)-1-((2-acetamidoethyl)thio)-4-methyl-1-oxopentan-2-yl 3-((tertbutoxycarbonyl)amino)-2,2-dimethylpropanoate (14b). To a solution of $\mathbf{1 3 b}$ in DMF $(0.1 \mathrm{M})$ was added $\mathrm{EDC} \mathrm{HCl}(1.5 \mathrm{eq})$ and $\mathrm{HOBt}(1.2 \mathrm{eq})$ and the reaction was stirred for $30 \mathrm{~min}$. NAc was added and stirred for $10 \mathrm{~min}$ prior to the addition of catalytic DMAP $(0.05$ eq). The reaction was stirred for 12 hours, diluted with water and EtOAc and the aqueous layer was extracted $(2 \times 40 \mathrm{~mL})$, organics combined, washed with sat. $\mathrm{NH}_{4} \mathrm{Cl}(2 \times 100 \mathrm{~mL})$, and dried over sodium sulfate. The organics were removed under reduced pressure and the remaining residue was purified by flash chromatography system $(1-10 \% \mathrm{MeOH} / \mathrm{DCM})$ to afford 22 as a white solid. Spectra was in accord with published literature. ${ }^{13}$ 


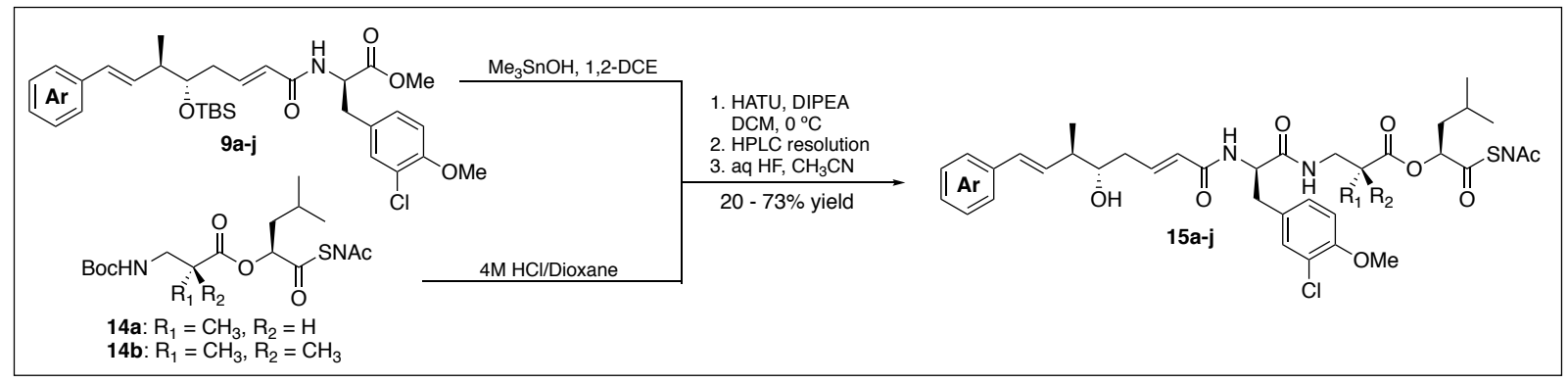

General Peptide Coupling Procedure: 9a-j (1 eq) was suspended in 1,2 dichloroethane $(0.2 \mathrm{M})$, treated with trimethyltin hydroxide (4 eq), and heat to $80^{\circ} \mathrm{C}$ for 4 hours. The reaction was cooled, diluted with $\mathrm{DCM}$ and washed with $1 \mathrm{~N} \mathrm{HCl}(2 \mathrm{x})$. The crude acid was used directly.

Simultaneously 14a or $14 \mathbf{b}(1.1 \mathrm{eq})$ was suspended in $4 \mathrm{M} \mathrm{HCl} / \mathrm{Dioxane}(5 \mathrm{~mL})$ and stirred for $1 \mathrm{~h}$ at $\mathrm{rt}$, concentrated, and used directly.

9a-m acid was suspended in DCM, cooled to $0{ }^{\circ} \mathrm{C}$, and treated with HATU (1.1 eq). 14a or 14b amine salt was suspended in DCM and treated with DIPEA $(2.5 \mathrm{eq})$, cooled to $0{ }^{\circ} \mathrm{C}$, and added to the mixture. The reaction was allowed to stir overnight, warming to $\mathrm{rt}$. The mixture was diluted with half saturated sodium bicarbonate, the aqueous layer extracted with DCM $(3 \times 10 \mathrm{~mL})$, organics combined, washed with brine, dried over $\mathrm{Na}_{2} \mathrm{SO}_{4}$, filtered, concentrated, and purified as described below. When utilized for analytical analysis, further HPLC purification was performed prior to deprotection on a HydroRP C18 $(250 \times 10.0$ $\mathrm{mm}, 4$ micron). HPLC conditions were as follows: mobile phase $(\mathrm{A}=$ deionized water $+0.1 \%$ formic acid, $\mathrm{B}=100 \%$ acetonitrile); $30 \% \mathrm{~B}$ for 2 minutes, $30 \%$ to $80 \% \mathrm{~B}$ over 48 minutes, $80 \% \mathrm{~B}$ for 5 minutes; 3.0 $\mathrm{mL} / \mathrm{min}$.

General Deprotection Procedure: The coupling product was suspended in acetonitrile $(0.1 \mathrm{M})$ in an open polypropylene vial. This was treated with $33 \%$ aq. HF (3 eq) and aloud to stir until the reaction was complete, as monitored by TLC $(2-5 \mathrm{~h})$. The reaction was diluted with DCM, and quenched by the slow addition of sat. $\mathrm{NaHCO}_{3}$ until the aqueous layer was $\mathrm{pH}=8$. The aqueous layer was then extracted with DCM ( 3 × $10 \mathrm{~mL})$, organics combined, washed with brine, dried over $\mathrm{Na}_{2} \mathrm{SO}_{4}$, filtered, concentrated, and purified as specified below. Diastereomeric ratios (dr) are reported as seen by NMR after deprotection.

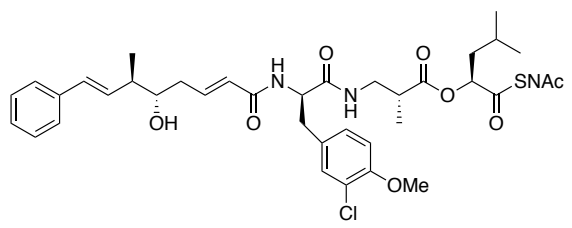

(S)-1-((2-acetamidoethyl)thio)-4-methyl-1-oxopentan-2-yl $\quad(R)-3-$ ((R)-3-(3-chloro-4-methoxyphenyl)-2-((2E,5S,6R,7E)-5-hydroxy-6methyl-8-phenylocta-2,7-dienamido)propanamido)-2-

methylpropanoate (15a). Reaction was run as per general coupling procedure and purified by flash chromatography system (1-10\% $\mathrm{MeOH} / \mathrm{DCM}) \mathrm{R}_{\mathrm{f}}=0.5(5 \% \mathrm{MeOH} / \mathrm{DCM}), 10: 1 \mathrm{dr}$. This was then deprotected as per general deprotection procedure and purified by flash chromatography system $(2-14 \% \mathrm{MeOH} / \mathrm{DCM})$ to afford 15a $(0.051 \mathrm{~g}$, $42 \%$ yield over 3 steps) as a clear and colorless oil: $\mathrm{R}_{\mathrm{f}}=0.35(10 \% \mathrm{MeOH} / \mathrm{DCM}) .{ }^{1} \mathbf{H}$ NMR: $(600 \mathrm{MHz}$, $\left.\mathrm{CD}_{3} \mathrm{OD}\right) \delta 7.37(\mathrm{~d}, \mathrm{~J}=7.0 \mathrm{~Hz}, 2 \mathrm{H}), 7.27(\mathrm{t}, \mathrm{J}=7.9 \mathrm{~Hz}, 2 \mathrm{H}), 7.25(\mathrm{~d}, \mathrm{~J}=2.2 \mathrm{~Hz}, 1 \mathrm{H}), 7.18(\mathrm{t}, \mathrm{J}=7.3 \mathrm{~Hz}$, $1 \mathrm{H}), 7.14(\mathrm{dd}, \mathrm{J}=8.4,2.2 \mathrm{~Hz}, 1 \mathrm{H}), 6.97(\mathrm{~d}, \mathrm{~J}=8.4 \mathrm{~Hz}, 1 \mathrm{H}), 6.81(\mathrm{dt}, \mathrm{J}=15.1,7.3 \mathrm{~Hz}, 1 \mathrm{H}), 6.41(\mathrm{~d}, \mathrm{~J}=$ $15.9 \mathrm{~Hz}, 1 \mathrm{H}), 6.23(\mathrm{dd}, \mathrm{J}=15.9,8.5 \mathrm{~Hz}, 1 \mathrm{H}), 6.01(\mathrm{~d}, \mathrm{~J}=15.4 \mathrm{~Hz}, 1 \mathrm{H}), 5.21(\mathrm{dd}, \mathrm{J}=9.6,4.0 \mathrm{~Hz}, 1 \mathrm{H}), 4.58$ $(\mathrm{dd}, \mathrm{J}=8.1,7.0 \mathrm{~Hz}, 1 \mathrm{H}), 3.83(\mathrm{~s}, 3 \mathrm{H}), 3.65(\mathrm{dt}, \mathrm{J}=8.7,4.6 \mathrm{~Hz}, 1 \mathrm{H}), 3.48(\mathrm{dd}, \mathrm{J}=13.5,6.6 \mathrm{~Hz}, 1 \mathrm{H}), 3.34-$ $3.27(\mathrm{~m}, 2 \mathrm{H}), 3.19(\mathrm{dd}, \mathrm{J}=13.5,7.0 \mathrm{~Hz}, 1 \mathrm{H}), 3.06-2.97(\mathrm{~m}, 3 \mathrm{H}), 2.85(\mathrm{dd}, \mathrm{J}=13.7,8.1 \mathrm{~Hz}, 1 \mathrm{H}), 2.70(\mathrm{~h}$, 
$\mathrm{J}=7.0 \mathrm{~Hz}, 1 \mathrm{H}), 2.44-2.35(\mathrm{~m}, 2 \mathrm{H}), 2.35-2.28(\mathrm{~m}, 1 \mathrm{H}), 1.91(\mathrm{~s}, 3 \mathrm{H}), 1.80-1.74(\mathrm{~m}, 1 \mathrm{H}), 1.74-1.69$ $(\mathrm{m}, 1 \mathrm{H}), 1.66-1.59(\mathrm{~m}, 1 \mathrm{H}), 1.15(\mathrm{~d}, \mathrm{~J}=6.9 \mathrm{~Hz}, 3 \mathrm{H}), 1.10(\mathrm{~d}, \mathrm{~J}=7.1 \mathrm{~Hz}, 3 \mathrm{H}), 0.94(\mathrm{~d}, \mathrm{~J}=6.5 \mathrm{~Hz}, 3 \mathrm{H})$, $0.91(\mathrm{~d}, \mathrm{~J}=6.5 \mathrm{~Hz}, 3 \mathrm{H}) .{ }^{13} \mathrm{C}$ NMR: (150 MHz, CD $\left.3 \mathrm{OD}\right) 200.3,175.1,173.5,173.4,168.1,155.4,143.5$, $139.1,132.5,132.1,131.9,131.5,129.8,129.5,128.1,127.1,126.1,123.2,113.4,78.6,75.3,56.6,56.2$, 44.2, 42.7, 41.9, 40.6, 39.8, 38.8, 38.1, 28.6, 25.7, 23.5, 22.5, 22.0, 17.5, 14.9; HRMS (ESI) calcd for $\mathrm{C}_{39} \mathrm{H}_{52} \mathrm{ClN}_{3} \mathrm{O}_{8} \mathrm{~S}[\mathrm{M}+\mathrm{H}]$ 758.3236, found 758.3238 .

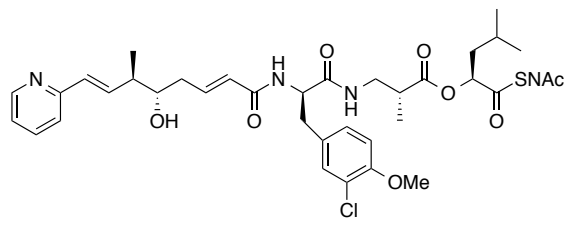

(S)-1-((2-acetamidoethyl)thio)-4-methyl-1-oxopentan-2-yl $\quad(R)-3-$ ((R)-3-(3-chloro-4-methoxyphenyl)-2-((2E,5S,6R,7E)-5-hydroxy-6methyl-8-(pyridin-2-yl)octa-2,7-dienamido)propanamido)-2methylpropanoate (15b). Reaction was run as per general coupling procedure, and purified by flash chromatography system (1-10\% $\mathrm{MeOH} / \mathrm{DCM}) \mathrm{R}_{\mathrm{f}}=0.65(10 \% \mathrm{MeOH} / \mathrm{DCM}), 7: 1 \mathrm{dr}$. This was then deprotected as per general deprotection procedure and purified by flash chromatography system $(2-15 \% \mathrm{MeOH} / \mathrm{DCM})$ to afford $\mathbf{1 5 b}(0.025 \mathrm{~g}$, $38 \%$ yield over 3 steps) as a clear and colorless oil: $\mathrm{R}_{\mathrm{f}}=0.25(10 \% \mathrm{MeOH} / \mathrm{DCM}) ;{ }^{1} \mathbf{H} \mathbf{~ N M R}:(600 \mathrm{MHz}$, $\left.\mathrm{CD}_{3} \mathrm{OD}\right) \delta 8.44(\mathrm{~d}, \mathrm{~J}=4.1 \mathrm{~Hz}, 1 \mathrm{H}), 7.76(\mathrm{td}, \mathrm{J}=7.7,1.9 \mathrm{~Hz}, 1 \mathrm{H}), 7.51(\mathrm{~d}, \mathrm{~J}=8.1 \mathrm{~Hz}, 1 \mathrm{H}), 7.26(\mathrm{~d}, \mathrm{~J}=2.3$ $\mathrm{Hz}, 1 \mathrm{H}), 7.25-7.22(\mathrm{~m}, 1 \mathrm{H}), 7.14(\mathrm{dd}, \mathrm{J}=8.4,2.2 \mathrm{~Hz}, 1 \mathrm{H}), 6.97(\mathrm{~d}, \mathrm{~J}=8.4 \mathrm{~Hz}, 1 \mathrm{H}), 6.81(\mathrm{dt}, \mathrm{J}=15.0,7.3$ $\mathrm{Hz}, 1 \mathrm{H}), 6.67(\mathrm{dd}, \mathrm{J}=16.0,8.4 \mathrm{~Hz}, 1 \mathrm{H}), 6.52(\mathrm{~d}, \mathrm{~J}=16.0 \mathrm{~Hz}, 1 \mathrm{H}), 6.02(\mathrm{~d}, \mathrm{~J}=15.4 \mathrm{~Hz}, 1 \mathrm{H}), 5.21(\mathrm{dd}, \mathrm{J}=$ 9.5, $4.0 \mathrm{~Hz}, 1 \mathrm{H}), 4.58(\mathrm{dd}, \mathrm{J}=8.1,6.9 \mathrm{~Hz}, 1 \mathrm{H}), 3.84(\mathrm{~s}, 3 \mathrm{H}), 3.68(\mathrm{dt}, \mathrm{J}=8.7,4.7 \mathrm{~Hz}, 1 \mathrm{H}), 3.48(\mathrm{dd}, \mathrm{J}=$ 13.5, 6.6 Hz, 1H), $3.33-3.30(\mathrm{~s}, 2 \mathrm{H}), 3.21(\mathrm{dd}, \mathrm{J}=13.5,7.0 \mathrm{~Hz}, 1 \mathrm{H}), 3.05-2.99(\mathrm{~m}, 3 \mathrm{H}), 2.85(\mathrm{dd}, \mathrm{J}=$ 13.8, 8.1 Hz, 1H), $2.71(\mathrm{~h}, \mathrm{~J}=7.0 \mathrm{~Hz}, 1 \mathrm{H}), 2.51-2.44(\mathrm{~m}, 1 \mathrm{H}), 2.44-2.37(\mathrm{~m}, 1 \mathrm{H}), 2.37-2.29(\mathrm{~m}, 1 \mathrm{H})$, $1.91(\mathrm{~s}, 3 \mathrm{H}), 1.81-1.69(\mathrm{~m}, 2 \mathrm{H}), 1.67-1.59(\mathrm{~m}, 1 \mathrm{H}), 1.18(\mathrm{~d}, \mathrm{~J}=6.9 \mathrm{~Hz}, 3 \mathrm{H}), 1.11(\mathrm{~d}, \mathrm{~J}=7.2 \mathrm{~Hz}, 3 \mathrm{H})$, $0.95(\mathrm{~d}, \mathrm{~J}=6.5 \mathrm{~Hz}, 3 \mathrm{H}), 0.92(\mathrm{~d}, \mathrm{~J}=6.5 \mathrm{~Hz}, 3 \mathrm{H}) \cdot{ }^{13} \mathbf{C}$ NMR $\left(150 \mathrm{MHz}, \mathrm{CD}_{3} \mathrm{OD}\right) \delta 200.3,175.1,173.5$, $173.4,168.1,157.3,155.4,149.7,143.3,138.7,138.6,131.9,131.6,131.3,129.8,126.3,123.4,122.3$, $113.4,111.4,78.7,75.1,56.6,56.2,44.1,42.7,42.0,40.6,39.9,38.7,38.1,28.6,25.8,23.5,22.5,22.0$, 17.1, 14.9; HRMS (ESI) calcd for $\mathrm{C}_{38} \mathrm{H}_{51} \mathrm{ClN}_{4} \mathrm{O}_{8} \mathrm{~S}[\mathrm{M}+\mathrm{H}]$ 759.3189, found 759.3184.

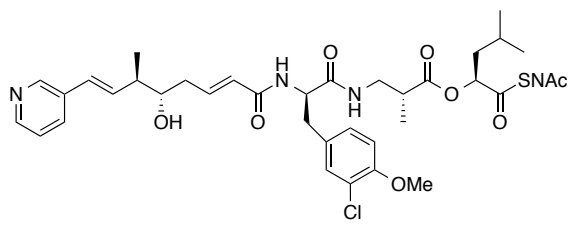

(S)-1-((2-acetamidoethyl)thio)-4-methyl-1-oxopentan-2-yl $\quad(R)-3-$ $((R)-3-(3-c h l o r o-4-m e t h o x y p h e n y l)-2-((2 E, 5 S, 6 R, 7 E)-5-h y d r o x y-6-$ methyl-8-(pyridin-3-yl)octa-2,7-dienamido)propanamido)-2-

methylpropanoate (15c). Reaction was run as per general coupling procedure, and purified by flash chromatography system (1-10\% $\mathrm{MeOH} / \mathrm{DCM}) \mathrm{R}_{\mathrm{f}}=0.65(10 \% \mathrm{MeOH} / \mathrm{DCM}) 7: 1 \mathrm{dr}$. This was then deprotected as per general deprotection procedure and purified by flash chromatography system $(2-15 \% \mathrm{MeOH} / \mathrm{DCM}, 7: 1)$ to afford the final product $15 \mathrm{c}(0.037 \mathrm{~g}, 51 \%$ yield over 3 steps $)$ as a clear and colorless oil: $\mathrm{R}_{\mathrm{f}}=0.25(10 \% \mathrm{MeOH} / \mathrm{DCM})$; ${ }^{1}$ H NMR $\left(600 \mathrm{MHz}, \mathrm{CD}_{3} \mathrm{OD}\right) \delta 8.52(\mathrm{~d}, \mathrm{~J}=2.2 \mathrm{~Hz}, 1 \mathrm{H}), 8.36(\mathrm{dd}, \mathrm{J}=4.9,1.6 \mathrm{~Hz}, 1 \mathrm{H}), 7.89(\mathrm{dt}, \mathrm{J}=8.1$, $1.9 \mathrm{~Hz}, 1 \mathrm{H}), 7.37$ (ddd, J = 8.0, 4.9, $0.9 \mathrm{~Hz}, 1 \mathrm{H}), 7.26$ (d, J = 2.2 Hz, 1H), 7.14 (dd, J = 8.4, 2.3 Hz, 1H), $6.97(\mathrm{~d}, \mathrm{~J}=8.5 \mathrm{~Hz}, 1 \mathrm{H}), 6.81(\mathrm{dt}, \mathrm{J}=15.2,7.3 \mathrm{~Hz}, 1 \mathrm{H}), 6.46(\mathrm{~d}, \mathrm{~J}=16.1 \mathrm{~Hz}, 1 \mathrm{H}), 6.41(\mathrm{dd}, \mathrm{J}=16.0,7.9$ $\mathrm{Hz}, 1 \mathrm{H}), 6.02(\mathrm{~d}, \mathrm{~J}=15.4 \mathrm{~Hz}, 1 \mathrm{H}), 5.21(\mathrm{dd}, \mathrm{J}=9.5,4.0 \mathrm{~Hz}, 1 \mathrm{H}), 4.59(\mathrm{dd}, \mathrm{J}=8.0,6.8 \mathrm{~Hz}, 1 \mathrm{H}), 3.84(\mathrm{~s}$, $3 \mathrm{H}), 3.67(\mathrm{dt}, \mathrm{J}=8.0,4.6 \mathrm{~Hz}, 1 \mathrm{H}), 3.48(\mathrm{dd}, \mathrm{J}=13.5,6.6 \mathrm{~Hz}, 1 \mathrm{H}), 3.33-3.30(\mathrm{~m}, 2 \mathrm{H}) 3.20(\mathrm{dd}, \mathrm{J}=13.5$, $6.9 \mathrm{~Hz}, 1 \mathrm{H}), 3.04-3.00(\mathrm{~m}, 3 \mathrm{H}) 2.85(\mathrm{dd}, \mathrm{J}=13.7,8.1 \mathrm{~Hz}, 1 \mathrm{H}) 2.70(\mathrm{~h}, \mathrm{~J}=7.0 \mathrm{~Hz}, 1 \mathrm{H}), 2.51-2.36(\mathrm{~m}$, $2 \mathrm{H}), 2.36-2.28(\mathrm{~m}, 1 \mathrm{H}), 1.91(\mathrm{~s}, 3 \mathrm{H}), 1.83-1.68(\mathrm{~m}, 2 \mathrm{H}), 1.65-1.61(\mathrm{~m}, 1 \mathrm{H}), 1.17(\mathrm{~d}, \mathrm{~J}=6.9 \mathrm{~Hz}, 3 \mathrm{H})$, $1.10(\mathrm{~d}, \mathrm{~J}=7.1 \mathrm{~Hz}, 3 \mathrm{H}), 0.95(\mathrm{~d}, \mathrm{~J}=6.5 \mathrm{~Hz}, 3 \mathrm{H}), 0.92(\mathrm{~d}, \mathrm{~J}=6.5 \mathrm{~Hz}, 3 \mathrm{H}) ;{ }^{13} \mathbf{C} \mathbf{N M R}\left(150 \mathrm{MHz}, \mathrm{CD}_{3} \mathrm{OD}\right)$ $\delta 200.3,175.1,173.5,173.4,168.0,155.4,148.3,148.2,143.3,136.3,135.5,134.8,131.9,131.5,129.9$, $127.9,126.2,125.2,123.2,113.4,78.7,75.2,56.6,56.2,44.3,42.7,42.0,40.6,39.9,38.9,38.1,28.6,25.8$, 23.5, 22.5, 22.0, 17.3, 14.9; HRMS (ESI) calcd for $\mathrm{C}_{38} \mathrm{H}_{51} \mathrm{ClN}_{4} \mathrm{O}_{8} \mathrm{~S}[\mathrm{M}+\mathrm{H}]$ 759.3189, found 759.3192. 


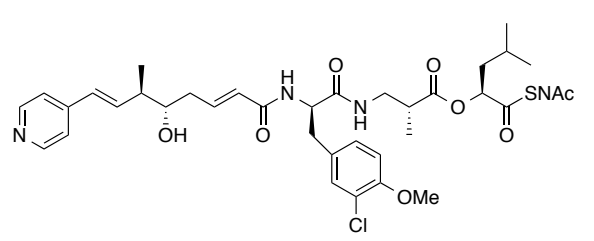

(S)-1-((2-acetamidoethyl)thio)-4-methyl-1-oxopentan-2-yl $\quad(R)-3-$ $((R)-3-(3-c h l o r o-4-m e t h o x y p h e n y l)-2-((2 E, 5 S, 6 R, 7 E)-5-h y d r o x y-6-$ methyl-8-(pyridin-4-yl)octa-2,7-dienamido)propanamido)-2-

methylpropanoate (15d). Reaction was run as per general coupling procedure, and purified by flash chromatography system $(1-10 \%$ $\mathrm{MeOH} / \mathrm{DCM}) \mathrm{R}_{\mathrm{f}}=0.65(10 \% \mathrm{MeOH} / \mathrm{DCM}), 11: 1 \mathrm{dr}$. This was then deprotected as per general deprotection procedure and purified by flash chromatography system $(2-15 \%$ $\mathrm{MeOH} / \mathrm{DCM})$ to afford the final product $15 \mathbf{d}(0.048 \mathrm{~g}, 49 \%$ yield over 3 steps $)$ as a clear and colorless oil: $\mathrm{R}_{\mathrm{f}}=0.25(10 \% \mathrm{MeOH} / \mathrm{DCM}) ;{ }^{1} \mathbf{H}$ NMR $\left(600 \mathrm{MHz}, \mathrm{CD}_{3} \mathrm{OD}\right) \delta 8.42(\mathrm{~d}, \mathrm{~J}=6.3 \mathrm{~Hz}, 1 \mathrm{H}), 7.40(\mathrm{~d}, \mathrm{~J}=6.3$ $\mathrm{Hz}, 1 \mathrm{H}), 7.25(\mathrm{~d}, \mathrm{~J}=2.2 \mathrm{~Hz}, 1 \mathrm{H}), 7.14(\mathrm{dd}, \mathrm{J}=8.5,2.2 \mathrm{~Hz}, 1 \mathrm{H}), 6.97(\mathrm{~d}, \mathrm{~J}=8.4 \mathrm{~Hz}, 1 \mathrm{H}), 6.80(\mathrm{dt}, \mathrm{J}=15.0$, $7.3 \mathrm{~Hz}, 1 \mathrm{H}), 6.61(\mathrm{dd}, \mathrm{J}=16.0,8.5 \mathrm{~Hz}, 1 \mathrm{H}), 6.44(\mathrm{~d}, \mathrm{~J}=15.9 \mathrm{~Hz}, 1 \mathrm{H}), 6.01(\mathrm{~d}, \mathrm{~J}=15.4 \mathrm{~Hz}, 1 \mathrm{H}), 5.21(\mathrm{dd}$, $\mathrm{J}=9.5,4.0 \mathrm{~Hz}, 1 \mathrm{H}), 4.58(\mathrm{dd}, \mathrm{J}=8.1,6.9 \mathrm{~Hz}, 1 \mathrm{H}), 3.84(\mathrm{~s}, 3 \mathrm{H}), 3.67(\mathrm{dt}, \mathrm{J}=7.9,4.7 \mathrm{~Hz}, 1 \mathrm{H}), 3.48(\mathrm{dd}, \mathrm{J}$ $=13.5,6.6 \mathrm{~Hz}, 1 \mathrm{H}), 3.33-3.30(\mathrm{~m}, 2 \mathrm{H}) 3.20(\mathrm{dd}, \mathrm{J}=13.5,7.0 \mathrm{~Hz}, 1 \mathrm{H}), 3.04-3.00(\mathrm{~m}, 3 \mathrm{H}), 2.85(\mathrm{dd}, \mathrm{J}=$ $13.8,8.1 \mathrm{~Hz}, 1 \mathrm{H}), 2.70(\mathrm{~h}, \mathrm{~J}=6.9 \mathrm{~Hz}, 1 \mathrm{H}), 2.51-2.42(\mathrm{~m}, 1 \mathrm{H}), 2.42-2.25(\mathrm{~m}, 2 \mathrm{H}), 1.91(\mathrm{~s}, 3 \mathrm{H}), 1.82-$ $1.68(\mathrm{~m}, 2 \mathrm{H}), 1.63(\mathrm{ddd}, \mathrm{J}=13.1,8.1,4.0 \mathrm{~Hz}, 1 \mathrm{H}), 1.17(\mathrm{~d}, \mathrm{~J}=6.8 \mathrm{~Hz}, 3 \mathrm{H}), 1.10(\mathrm{~d}, \mathrm{~J}=7.1 \mathrm{~Hz}, 3 \mathrm{H}), 0.94$ $(\mathrm{d}, \mathrm{J}=6.5 \mathrm{~Hz}, 3 \mathrm{H}), 0.92(\mathrm{~d}, \mathrm{~J}=6.5 \mathrm{~Hz}, 3 \mathrm{H}) .{ }^{13} \mathbf{C}$ NMR $\left(150 \mathrm{MHz}, \mathrm{CD}_{3} \mathrm{OD}\right) \delta 200.3,175.1,173.5,173.4$, $168.0,155.4,150.2,147.7,143.2,139.5,131.9,131.5,129.8,129.4,126.3,123.2,122.4,113.4,78.7,75.0$, 56.6, 56.2, 44.3, 42.8, 42.0, 40.6, 39.9, 38.9, 38.1, 28.6, 25.8, 23.5, 22.5, 21.99, 17.2, 14.9.; HRMS (ESI) calcd for $\mathrm{C}_{38} \mathrm{H}_{51} \mathrm{ClN}_{4} \mathrm{O}_{8} \mathrm{~S}[\mathrm{M}+\mathrm{H}]$ 759.3189, found 759.3187 .

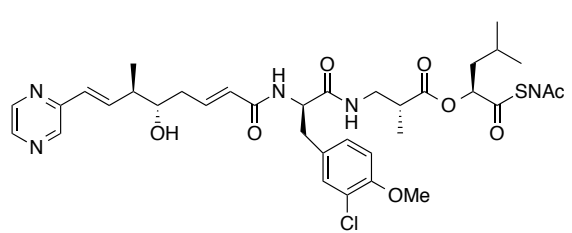

(S)-1-((2-acetamidoethyl)thio)-4-methyl-1-oxopentan-2-yl $\quad(R)-3-$ $((R)-3-(3-c h l o r o-4-m e t h o x y p h e n y l)-2-((2 E, 5 S, 6 R, 7 E)-5-h y d r o x y-6-$ methyl-8-(pyrazin-2-yl)octa-2,7-dienamido)propanamido)-2methylpropanoate (15e). Reaction was run as per general coupling procedure, and purified by flash chromatography system $(1-10 \%$ $\mathrm{MeOH} / \mathrm{DCM}) \mathrm{R}_{\mathrm{f}}=0.65(10 \% \mathrm{MeOH} / \mathrm{DCM}), 7: 1 \mathrm{dr}$. This was then deprotected as per general deprotection procedure and purified by flash chromatography system $(2-15 \% \mathrm{MeOH} / \mathrm{DCM})$ to afford the final product 15e $(0.021 \mathrm{~g}, 25 \%$ yield over 3 steps $)$ as a clear and colorless oil: $R_{\mathrm{f}}=0.25(10 \% \mathrm{MeOH} / \mathrm{DCM}) ;{ }^{1} \mathbf{H} \mathbf{N M R}$ $\left(400 \mathrm{MHz}, \mathrm{CD}_{3} \mathrm{OD}\right) \delta 8.62(\mathrm{~s}, 1 \mathrm{H}), 8.51(\mathrm{~s}, 1 \mathrm{H}), 8.39(\mathrm{~d}, \mathrm{~J}=2.6 \mathrm{~Hz}, 1 \mathrm{H}), 7.26(\mathrm{~d}, \mathrm{~J}=2.0 \mathrm{~Hz}, 1 \mathrm{H}), 7.14$ $(\mathrm{dd}, \mathrm{J}=8.5,2.1 \mathrm{~Hz}, 1 \mathrm{H}), 6.97(\mathrm{~d}, \mathrm{~J}=8.4 \mathrm{~Hz}, 1 \mathrm{H}), 6.93(\mathrm{dd}, \mathrm{J}=15.9,8.5 \mathrm{~Hz}, 1 \mathrm{H}), 6.81(\mathrm{dt}, \mathrm{J}=14.9,7.3$ $\mathrm{Hz}, 1 \mathrm{H}), 6.57(\mathrm{~d}, \mathrm{~J}=16.0 \mathrm{~Hz}, 1 \mathrm{H}), 6.02(\mathrm{~d}, \mathrm{~J}=15.4 \mathrm{~Hz}, 1 \mathrm{H}), 5.21(\mathrm{dd}, \mathrm{J}=9.4,3.9 \mathrm{~Hz}, 1 \mathrm{H}), 4.58(\mathrm{t}, \mathrm{J}=7.5$ $\mathrm{Hz}, 1 \mathrm{H}), 3.69(\mathrm{dt}, \mathrm{J}=9.0,4.7 \mathrm{~Hz}, 1 \mathrm{H}), 3.48(\mathrm{dd}, \mathrm{J}=13.4,6.6 \mathrm{~Hz}, 1 \mathrm{H}), 3.37-3.24(\mathrm{~m}, 2 \mathrm{H}), 3.20(\mathrm{dd}, \mathrm{J}=$ 13.5, 7.1 Hz, 1H), $3.07-2.97(\mathrm{~m}, 1 \mathrm{H}), 3.03(\mathrm{t}, \mathrm{J}=6.8 \mathrm{~Hz}, 2 \mathrm{H}), 2.85$ (dd, J = 13.7, 8.2 Hz, 1H), 2.70 (h, J $=6.9 \mathrm{~Hz}, 1 \mathrm{H}), 2.56-2.42(\mathrm{~m}, 1 \mathrm{H}), 2.41-2.25(\mathrm{~m}, 2 \mathrm{H}) 1.91(\mathrm{~s}, 3 \mathrm{H}), 1.82-1.68(\mathrm{~m}, 2 \mathrm{H}), 1.62(\mathrm{ddd}, \mathrm{J}=$ 13.1, 8.1, 3.9 Hz, 1H), 1.19 (d, J = 6.8 Hz, 3H), 1.10 (d, J = 7.0 Hz, 3H), 0.95 (d, J = 6.3 Hz, 3H), $0.92(\mathrm{~d}$, $\mathrm{J}=6.3 \mathrm{~Hz}, 3 \mathrm{H}) .{ }^{13} \mathbf{C}$ NMR $\left(151 \mathrm{MHz}, \mathrm{CD}_{3} \mathrm{OD}\right) \delta 200.3,175.1,173.5,173.4,168.0,155.4,153.1,145.4$, $143.9,143.5,143.2,141.4,131.9,131.5,129.9,128.2,126.3,123.2,113.4,78.7,75.0,56.6,56.2$, 44.2, $42.8,42.0,40.6,39.9,38.8,38.1,28.6,25.8,23.5,22.5,22.0,17.0,14.9$. HRMS (ESI) calcd for $\mathrm{C}_{37} \mathrm{H}_{50} \mathrm{ClN}_{5} \mathrm{O}_{8} \mathrm{~S}[\mathrm{M}+\mathrm{H}] 760.3141$, found 760.3143 .

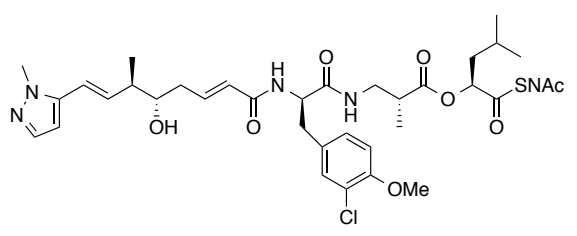

$(S)-1-((2-a c e t a m i d o e t h y l) t h i o)-4-m e t h y l-1-o x o p e n t a n-2-y l \quad(R)-3-$ ((R)-3-(3-chloro-4-methoxyphenyl)-2-((2E,5S,6R,7E)-5-hydroxy-6methyl-8-(1-methyl-1H-pyrazol-5-yl)octa-2,7-

dienamido)propanamido)-2-methylpropanoate (15f). Reaction was run as per general coupling procedure, and purified by flash chromatography system $(1-10 \% \mathrm{MeOH} / \mathrm{DCM}) \mathrm{R}_{\mathrm{f}}=0.65(10 \% \mathrm{MeOH} / \mathrm{DCM}), 9: 1 \mathrm{dr}$. This was then deprotected as per general deprotection procedure and purified by flash chromatography system $(2-15 \%$ $\mathrm{MeOH} / \mathrm{DCM})$ to afford the final product $\mathbf{1 5 f}(0.022,29 \%$ yield over 3 steps $)$ as a clear and colorless oil: $\mathrm{R}_{\mathrm{f}}$ $=0.25(10 \% \mathrm{MeOH} / \mathrm{DCM}) ;{ }^{1} \mathbf{H}$ NMR $\left(600 \mathrm{MHz}, \mathrm{CD}_{3} \mathrm{OD}\right) \delta 7.34(\mathrm{~d}, \mathrm{~J}=2.2 \mathrm{~Hz}, 1 \mathrm{H}), 7.25(\mathrm{~d}, \mathrm{~J}=2.2 \mathrm{~Hz}$, 
1H), $7.14(\mathrm{dd}, \mathrm{J}=8.4,2.2 \mathrm{~Hz}, 1 \mathrm{H}), 6.97(\mathrm{~d}, \mathrm{~J}=8.5 \mathrm{~Hz}, 1 \mathrm{H}), 6.81(\mathrm{dt}, \mathrm{J}=15.0,7.3 \mathrm{~Hz}, 1 \mathrm{H}), 6.42(\mathrm{~d}, \mathrm{~J}=$ $15.9 \mathrm{~Hz}, 1 \mathrm{H}), 6.38(\mathrm{~d}, \mathrm{~J}=2.1 \mathrm{~Hz}, 1 \mathrm{H}), 6.25(\mathrm{dd}, \mathrm{J}=15.9,8.6 \mathrm{~Hz}, 1 \mathrm{H}), 6.01(\mathrm{dd}, \mathrm{J}=15.4,1.5 \mathrm{~Hz}, 1 \mathrm{H}), 5.21$ $(\mathrm{dd}, \mathrm{J}=9.5,3.9 \mathrm{~Hz}, 1 \mathrm{H}), 4.58(\mathrm{dd}, \mathrm{J}=8.1,7.0 \mathrm{~Hz}, 1 \mathrm{H}), 3.84(\mathrm{~s}, 3 \mathrm{H}), 3.82(\mathrm{~s}, 3 \mathrm{H}), 3.65$ (dt, J = 8.0, $4.6 \mathrm{~Hz}$, $1 \mathrm{H}), 3.48(\mathrm{dd}, \mathrm{J}=13.5,6.6 \mathrm{~Hz}, 1 \mathrm{H}), 3.33-3.30(\mathrm{~m}, 2 \mathrm{H}) 3.20(\mathrm{dd}, \mathrm{J}=13.5,7.0 \mathrm{~Hz}, 1 \mathrm{H}), 3.03(\mathrm{td}, \mathrm{J}=6.6$, $1.8 \mathrm{~Hz}, 2 \mathrm{H}), 3.03-3.00(\mathrm{~m}, 1 \mathrm{H}) 2.85(\mathrm{dd}, \mathrm{J}=13.8,8.1 \mathrm{~Hz}, 1 \mathrm{H}), 2.70(\mathrm{~h}, \mathrm{~J}=6.9 \mathrm{~Hz}, 1 \mathrm{H}), 2.50-2.36(\mathrm{~m}$, $2 \mathrm{H}), 2.36-2.28(\mathrm{~m}, 1 \mathrm{H}), 1.91(\mathrm{~s}, 3 \mathrm{H}), 1.82-1.68(\mathrm{~m}, 2 \mathrm{H}), 1.63(\mathrm{ddd}, \mathrm{J}=13.2,8.1,3.9 \mathrm{~Hz}, 1 \mathrm{H}), 1.15(\mathrm{~d}$, $\mathrm{J}=6.9 \mathrm{~Hz}, 3 \mathrm{H}), 1.10(\mathrm{~d}, \mathrm{~J}=7.1 \mathrm{~Hz}, 3 \mathrm{H}), 0.95(\mathrm{~d}, \mathrm{~J}=6.4 \mathrm{~Hz}, 3 \mathrm{H}), 0.92(\mathrm{~d}, \mathrm{~J}=6.5 \mathrm{~Hz}, 3 \mathrm{H}) .{ }^{13} \mathbf{C} \mathbf{N M R}:(150$ $\left.\mathrm{MHz}, \mathrm{CD}_{3} \mathrm{OD}\right) \delta 200.3,175.1,173.5,173.4,168.0,155.4,143.3,142.8,139.1,137.9,131.9,131.5,129.8$, 126.2, 123.2, 118.4, 113.4, 103.56, 78.7, 75.0, 56.6, 56.2, 44.3, 42.7, 42.0, 40.6, 39.9, 38.9, 38.1, 28.6, 25.7, 23.5, 22.5, 22.0, 17.3, 14.9; HRMS (ESI) calcd for $\mathrm{C}_{37} \mathrm{H}_{52} \mathrm{ClN}_{5} \mathrm{O}_{8} \mathrm{~S}$ [M+H] 762.3298, found 762.3295.

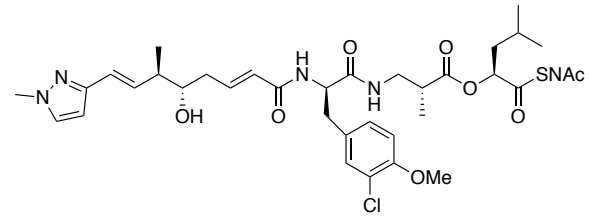

(S)-1-((2-acetamidoethyl)thio)-4-methyl-1-oxopentan-2-yl $(R)-3-$ ((R)-3-(3-chloro-4-methoxyphenyl)-2-((2E,5S,6R,7E)-5-hydroxy6-methyl-8-(1-methyl-1H-pyrazol-3-yl)octa-2,7-dienamido) propanamido)-2-methylpropanoate (15g). Reaction was run as per general coupling procedure, and purified by flash chromatography (1$10 \% \mathrm{MeOH} / \mathrm{DCM}) \mathrm{R}_{\mathrm{f}}=0.65(10 \% \mathrm{MeOH} / \mathrm{DCM}), 11: 1 \mathrm{dr}$. This was then deprotected as per general deprotection procedure and purified by flash chromatography $(2-15 \% \mathrm{MeOH} / \mathrm{DCM})$ to afford the final product $15 \mathrm{~g}(0.019 \mathrm{~g}, 34 \%$ yield over 3 steps $)$ as a clear and colorless oil: $\mathrm{R}_{\mathrm{f}}=0.25(10 \% \mathrm{MeOH} / \mathrm{DCM})$; ${ }^{1} \mathbf{H}$ NMR $\left(600 \mathrm{MHz}, \mathrm{CD}_{3} \mathrm{OD}\right) \delta 7.46(\mathrm{~d}, \mathrm{~J}=2.3 \mathrm{~Hz}, 1 \mathrm{H}), 7.26(\mathrm{~d}, \mathrm{~J}=2.1 \mathrm{~Hz}, 1 \mathrm{H}), 7.14(\mathrm{dd}, \mathrm{J}=8.5,2.2 \mathrm{~Hz}$, $1 \mathrm{H}), 6.97(\mathrm{~d}, \mathrm{~J}=8.4 \mathrm{~Hz}, 1 \mathrm{H}), 6.79(\mathrm{dt}, \mathrm{J}=15.1,7.3 \mathrm{~Hz}, 1 \mathrm{H}), 6.34(\mathrm{~d}, \mathrm{~J}=2.4 \mathrm{~Hz}, 1 \mathrm{H}), 6.32(\mathrm{~d}, \mathrm{~J}=16.6 \mathrm{~Hz}$, $1 \mathrm{H}), 6.21(\mathrm{dd}, \mathrm{J}=16.1,8.4 \mathrm{~Hz}, 1 \mathrm{H}), 6.01(\mathrm{~d}, \mathrm{~J}=15.4 \mathrm{~Hz}, 1 \mathrm{H}), 5.21(\mathrm{dd}, \mathrm{J}=9.5,4.0 \mathrm{~Hz}, 1 \mathrm{H}), 4.58(\mathrm{dd}, \mathrm{J}=$ 8.1, $7.0 \mathrm{~Hz}, 1 \mathrm{H}), 3.84(\mathrm{~s}, 6 \mathrm{H}), 3.63(\mathrm{dt}, \mathrm{J}=8.7,4.6 \mathrm{~Hz}, 1 \mathrm{H}), 3.48(\mathrm{dd}, \mathrm{J}=13.5,6.6 \mathrm{~Hz}, 1 \mathrm{H}), 3.33-3.29$ $(\mathrm{m}, 2 \mathrm{H}), 3.20(\mathrm{dd}, \mathrm{J}=13.5,7.0 \mathrm{~Hz}, 1 \mathrm{H}), 3.05-2.99(\mathrm{~m}, 3 \mathrm{H}), 2.85(\mathrm{dd}, \mathrm{J}=13.7,8.1 \mathrm{~Hz}, 1 \mathrm{H}), 2.70(\mathrm{~h}, \mathrm{~J}=$ $7.0 \mathrm{~Hz}, 1 \mathrm{H}), 2.41-2.33(\mathrm{~m}, 2 \mathrm{H}), 2.30(\mathrm{dt}, \mathrm{J}=15.4,7.8 \mathrm{~Hz}, 1 \mathrm{H}), 1.91(\mathrm{~s}, 3 \mathrm{H}), 1.82-1.68(\mathrm{~m}, 2 \mathrm{H}), 1.63$ $(\mathrm{ddd}, \mathrm{J}=13.2,8.1,3.9 \mathrm{~Hz}, 1 \mathrm{H}), 1.13(\mathrm{~d}, \mathrm{~J}=6.9 \mathrm{~Hz}, 3 \mathrm{H}), 1.10(\mathrm{~d}, \mathrm{~J}=7.2 \mathrm{~Hz}, 3 \mathrm{H}), 0.95(\mathrm{~d}, \mathrm{~J}=6.5 \mathrm{~Hz}, 3 \mathrm{H})$, $0.92(\mathrm{~d}, \mathrm{~J}=6.4 \mathrm{~Hz}, 3 \mathrm{H}) .{ }^{13} \mathrm{C}$ NMR: $\left(150 \mathrm{MHz}, \mathrm{CD}_{3} \mathrm{OD}\right) \delta 200.3,175.1,173.5,173.4,168.1,155.4,152.2$, $143.4,134.5,133.0,131.9,131.5,129.9,126.2,123.6,123.2,113.4,103.3,78.6,75.2,56.6,56.3,44.0$, 42.7, 42.0, 40.6, 39.9, 38.7, 38.6, 38.1, 28.6, 25.8, 23.5, 22.5, 22.0, 17.2, 14.9. HRMS (ESI) calcd for $\mathrm{C}_{37} \mathrm{H}_{52} \mathrm{ClN}_{5} \mathrm{O}_{8} \mathrm{~S}[\mathrm{M}+\mathrm{H}]$ 762.3298, found 762.3295 .

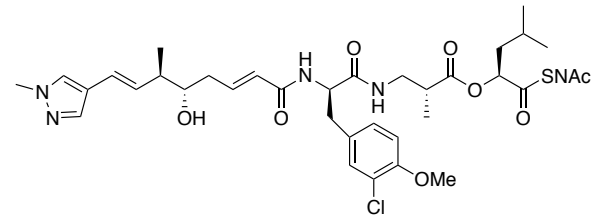

(S)-1-((2-acetamidoethyl)thio)-4-methyl-1-oxopentan-2-yl $(R)-3-$ $((R)-3-(3-c h l o r o-4-m e t h o x y p h e n y l)-2-((2 E, 5 S, 6 R, 7 E)-5-h y d r o x y-$ 6-methyl-8-(1-methyl-1H-pyrazol-4-yl)octa-2,7dienamido)propanamido)-2-methylpropanoate (15h). Reaction was run as per general coupling procedure, and purified by flash chromatography system $(1-10 \% \mathrm{MeOH} / \mathrm{DCM}) \mathrm{R}_{\mathrm{f}}=0.65(10 \% \mathrm{MeOH} / \mathrm{DCM}), 11: 1 \mathrm{dr}$. This was then deprotected as per general deprotection procedure and purified by flash chromatography system $(2-15 \%$ $\mathrm{MeOH} / \mathrm{DCM})$ to afford the final product $15 \mathrm{~h}(0.065 \mathrm{~g}, 48 \%$ yield over 3 steps $)$ as a clear and colorless oil: $\mathrm{R}_{\mathrm{f}}=0.25(10 \% \mathrm{MeOH} / \mathrm{DCM}) ;{ }^{1} \mathbf{H}$ NMR $\left(600 \mathrm{MHz}, \mathrm{CD}_{3} \mathrm{OD}\right) \delta 7.54(\mathrm{~s}, 1 \mathrm{H}), 7.50(\mathrm{~s}, 1 \mathrm{H}), 7.25(\mathrm{~d}, \mathrm{~J}=2.1$ $\mathrm{Hz}, 1 \mathrm{H}), 7.13(\mathrm{dd}, \mathrm{J}=8.2,2.1 \mathrm{~Hz}, 1 \mathrm{H}), 6.97(\mathrm{~d}, \mathrm{~J}=8.3 \mathrm{~Hz}, 1 \mathrm{H}), 6.80(\mathrm{dt}, \mathrm{J}=15.0,7.3 \mathrm{~Hz}, 1 \mathrm{H}), 6.22(\mathrm{~d}, \mathrm{~J}$ $=16.0 \mathrm{~Hz}, 1 \mathrm{H}), 6.00(\mathrm{~d}, \mathrm{~J}=16.1,1 \mathrm{H}), 5.94(\mathrm{dd}, \mathrm{J}=16.0,8.4 \mathrm{~Hz}, 1 \mathrm{H}), 5.21(\mathrm{dd}, \mathrm{J}=9.5,4.0 \mathrm{~Hz}, 1 \mathrm{H}), 4.58$ $(\mathrm{t}, \mathrm{J}=7.5 \mathrm{~Hz}, 1 \mathrm{H}), 3.84(\mathrm{~s}, 3 \mathrm{H}), 3.84(\mathrm{~s}, 3 \mathrm{H}), 3.60(\mathrm{dt}, \mathrm{J}=8.5,4.5 \mathrm{~Hz}, 1 \mathrm{H}), 3.48(\mathrm{dd}, \mathrm{J}=13.5,6.6 \mathrm{~Hz}, 1 \mathrm{H})$, $3.33-3.30(\mathrm{~m}, 2 \mathrm{H}), 3.20(\mathrm{dd}, \mathrm{J}=13.5,6.9 \mathrm{~Hz}, 1 \mathrm{H}), 3.03(\mathrm{t}, \mathrm{J}=5.8 \mathrm{~Hz}, 2 \mathrm{H}), 3.03-3.00(\mathrm{~m}, 1 \mathrm{H}), 2.85$ (dd, $\mathrm{J}=13.7,8.2 \mathrm{~Hz}, 1 \mathrm{H}), 2.70(\mathrm{~h}, \mathrm{~J}=7.0 \mathrm{~Hz}, 1 \mathrm{H}), 2.40-2.23(\mathrm{~m}, 3 \mathrm{H}), 1.91(\mathrm{~s}, 3 \mathrm{H}), 1.81-1.68(\mathrm{~m}, 2 \mathrm{H}), 1.63$ $(\mathrm{td}, \mathrm{J}=9.2,8.6,4.4 \mathrm{~Hz}, 1 \mathrm{H}), 1.10(\mathrm{~d}, \mathrm{~J}=7.0 \mathrm{~Hz}, 6 \mathrm{H}), 0.95(\mathrm{~d}, \mathrm{~J}=6.3 \mathrm{~Hz}, 3 \mathrm{H}), 0.92(\mathrm{~d}, \mathrm{~J}=6.3 \mathrm{~Hz}, 3 \mathrm{H}) .{ }^{13} \mathrm{C}$ NMR $\left(150 \mathrm{MHz}, \mathrm{CD}_{3} \mathrm{OD}\right) \delta 200.3,175.1,173.5,173.4,168.1,155.4,143.6,137.7,131.9,131.5,130.9$, $129.8,129.4,126.0,123.2,122.5,121.6,113.4,78.7,75.4,56.6,56.2,44.1,42.7,42.0,40.6,39.9,38.7$, 
38.6, 38.1 , 28.6, 25.8, 23.5, 22.5, 22.0, 17.4, 14.9. HRMS (ESI) calcd for $\mathrm{C}_{37} \mathrm{H}_{52} \mathrm{ClN}_{5} \mathrm{O}_{8} \mathrm{~S}[\mathrm{M}+\mathrm{H}]$ 762.3298, found 762.3294 .

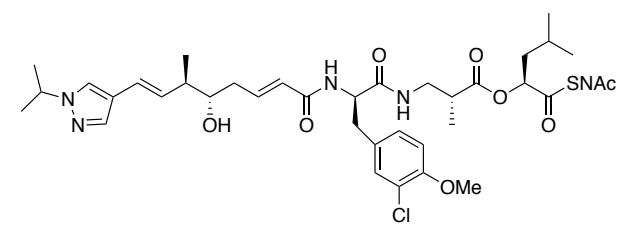
chromatography (1-10\% MeOH/DCM) $\mathrm{R}_{\mathrm{f}}=0.65(10 \% \mathrm{MeOH} / \mathrm{DCM}), 11: 1 \mathrm{dr}$. This was then deprotected as per general deprotection procedure and purified by flash chromatography $(2-15 \% \mathrm{MeOH} / \mathrm{DCM})$ to afford the final product 15i $(0.025,38 \%$ yield over 3 steps $)$ as a clear and colorless oil: $\mathrm{R}_{\mathrm{f}}=0.25(10 \%$ $\mathrm{MeOH} / \mathrm{DCM}) ;{ }^{1} \mathbf{H}$ NMR $\left(600 \mathrm{MHz}, \mathrm{CD}_{3} \mathrm{OD}\right) \delta 7.64(\mathrm{~s}, 1 \mathrm{H}), 7.51(\mathrm{~s}, 1 \mathrm{H}), 7.25(\mathrm{~d}, \mathrm{~J}=2.2 \mathrm{~Hz}, 1 \mathrm{H}), 7.14$ $(\mathrm{dd}, \mathrm{J}=8.4,2.2 \mathrm{~Hz}, 1 \mathrm{H}), 6.97(\mathrm{~d}, \mathrm{~J}=8.4 \mathrm{~Hz}, 1 \mathrm{H}), 6.80(\mathrm{dt}, \mathrm{J}=15.0,7.3 \mathrm{~Hz}, 1 \mathrm{H}), 6.23(\mathrm{~d}, \mathrm{~J}=16.0 \mathrm{~Hz}, 1 \mathrm{H})$, $6.00(\mathrm{~d}, \mathrm{~J}=15.4 \mathrm{~Hz}, 1 \mathrm{H}), 5.94(\mathrm{dd}, \mathrm{J}=16.0,8.5 \mathrm{~Hz}, 1 \mathrm{H}), 5.21(\mathrm{dd}, \mathrm{J}=9.5,4.0 \mathrm{~Hz}, 1 \mathrm{H}), 4.58(\mathrm{t}, \mathrm{J}=7.5 \mathrm{~Hz}$, $1 \mathrm{H}), 4.46$ (hept, $\mathrm{J}=6.6 \mathrm{~Hz}, 1 \mathrm{H}), 3.84(\mathrm{~s}, 3 \mathrm{H}), 3.61(\mathrm{dt}, \mathrm{J}=8.6,4.5 \mathrm{~Hz}, 1 \mathrm{H}), 3.48(\mathrm{dd}, \mathrm{J}=13.5,6.6 \mathrm{~Hz}, 1 \mathrm{H})$, $3.33-3.30(\mathrm{~m}, 2 \mathrm{H}), 3.20(\mathrm{dd}, \mathrm{J}=13.5,7.0 \mathrm{~Hz}, 1 \mathrm{H}), 3.03(\mathrm{t}, \mathrm{J}=6.6 \mathrm{~Hz}, 2 \mathrm{H}), 3.03-3.00(\mathrm{~m}, 1 \mathrm{H}), 2.85$ (dd, $\mathrm{J}=13.8,8.1 \mathrm{~Hz}, 1 \mathrm{H}), 2.70(\mathrm{~h}, \mathrm{~J}=6.9 \mathrm{~Hz}, 1 \mathrm{H}), 2.43-2.23(\mathrm{~m}, 3 \mathrm{H}), 1.91(\mathrm{~s}, 3 \mathrm{H}), 1.82-1.69(\mathrm{~m}, 2 \mathrm{H}), 1.63$ $(\mathrm{ddd}, \mathrm{J}=13.2,8.1,3.9 \mathrm{~Hz}, 1 \mathrm{H}), 1.47(\mathrm{~d}, \mathrm{~J}=6.7 \mathrm{~Hz}, 6 \mathrm{H}), 1.11(\mathrm{~d}, \mathrm{~J}=6.9 \mathrm{~Hz}, 3 \mathrm{H}), 1.10(\mathrm{~d}, \mathrm{~J}=7.1 \mathrm{~Hz}, 3 \mathrm{H})$, $0.95(\mathrm{~d}, \mathrm{~J}=6.4 \mathrm{~Hz}, 3 \mathrm{H}), 0.92(\mathrm{~d}, \mathrm{~J}=6.4 \mathrm{~Hz}, 3 \mathrm{H}) \cdot{ }^{13} \mathbf{C} \mathbf{N M R}\left(150 \mathrm{MHz}, \mathrm{CD}_{3} \mathrm{OD}\right) \delta 200.4,175.1,173.5$, $173.4,168.1,155.4,143.6,137.3,131.9,131.5,130.6,129.9,126.0$ (2), 123.2, 121.9, 121.8, 113.4, 78.7, 75.4, 56.6, 56.2, 55.0, 44.2, 42.7, 42.0, 40.6, 39.9, 38.7, 38.1, 28.6, 25.8, 23.5, 23.1, 22.5, 22.0, 17.4, 14.9. HRMS (ESI) calcd for $\mathrm{C}_{39} \mathrm{H}_{56} \mathrm{ClN}_{5} \mathrm{O}_{8} \mathrm{~S}[\mathrm{M}+\mathrm{H}]$ 790.3611, found 790.3608.

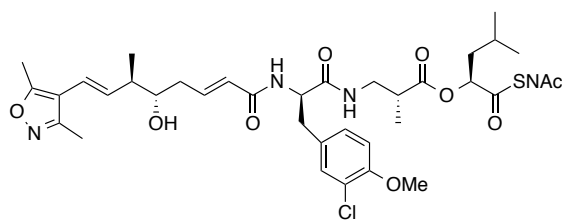

$(S)-1-((2-a c e t a m i d o e t h y l) t h i o)-4-m e t h y l-1-o x o p e n t a n-2-y l \quad(R)-3-$ ((R)-3-(3-chloro-4-methoxyphenyl)-2-((2E,5S,6R,7E)-8-(3,5dimethylisoxazol-4-yl)-5-hydroxy-6-methylocta-2,7-

dienamido)propanamido)-2-methylpropanoate (15j). Reaction was run as per general coupling procedure, and purified by flash chromatography (1-10\% MeOH/DCM) $\mathrm{R}_{\mathrm{f}}=0.65$ (10\% MeOH/DCM), 11:1 dr. This was then deprotected as per general deprotection procedure and purified by flash chromatography $(2-15 \% \mathrm{MeOH} / \mathrm{DCM})$ to afford the final product $\mathbf{1 5} \mathbf{j}(0.015 \mathrm{~g}, 43 \%$ yield over 3 steps $)$ as a clear and colorless oil: $\mathrm{R}_{\mathrm{f}}=0.25(10 \%$ $\mathrm{MeOH} / \mathrm{DCM}) ;{ }^{1} \mathbf{H}$ NMR $\left(600 \mathrm{MHz}, \mathrm{CD}_{3} \mathrm{OD}\right) \delta 7.25(\mathrm{~d}, \mathrm{~J}=2.1 \mathrm{~Hz}, 1 \mathrm{H}), 7.14(\mathrm{dd}, \mathrm{J}=8.4,2.2 \mathrm{~Hz}, 1 \mathrm{H})$, $6.97(\mathrm{~d}, \mathrm{~J}=8.4 \mathrm{~Hz}, 1 \mathrm{H}), 6.81(\mathrm{dt}, \mathrm{J}=15.0,7.3 \mathrm{~Hz}, 1 \mathrm{H}), 6.14(\mathrm{~d}, \mathrm{~J}=16.3 \mathrm{~Hz}, 1 \mathrm{H}), 6.01(\mathrm{~d}, \mathrm{~J}=15.4 \mathrm{~Hz}$, $1 \mathrm{H}), 5.93(\mathrm{dd}, \mathrm{J}=16.3,8.5 \mathrm{~Hz}, 1 \mathrm{H}), 5.21(\mathrm{dd}, \mathrm{J}=9.5,4.0 \mathrm{~Hz}, 1 \mathrm{H}), 4.57(\mathrm{t}, \mathrm{J}=7.5 \mathrm{~Hz}, 1 \mathrm{H}), 3.84(\mathrm{~s}, 3 \mathrm{H})$, $3.63(\mathrm{dt}, \mathrm{J}=8.5,4.7 \mathrm{~Hz}, 1 \mathrm{H}), 3.48(\mathrm{dd}, \mathrm{J}=13.5,6.6 \mathrm{~Hz}, 1 \mathrm{H}), 3.33-3.30(\mathrm{~m}, 2 \mathrm{H}) 3.20(\mathrm{dd}, \mathrm{J}=13.5,7.0$ $\mathrm{Hz}, 1 \mathrm{H}), 3.03(\mathrm{t}, \mathrm{J}=6.8 \mathrm{~Hz}, 2 \mathrm{H}), 3.02-2.99(\mathrm{~m}, 1 \mathrm{H}) 2.85(\mathrm{dd}, \mathrm{J}=13.7,8.1 \mathrm{~Hz}, 1 \mathrm{H}), 2.70(\mathrm{~h}, \mathrm{~J}=7.0 \mathrm{~Hz}$, $1 \mathrm{H}), 2.39(\mathrm{~s}, 3 \mathrm{H}), 2.38-2.30(\mathrm{~m}, 3 \mathrm{H}), 2.28(\mathrm{~s}, 3 \mathrm{H}), 1.91(\mathrm{~s}, 3 \mathrm{H}), 1.82-1.67(\mathrm{~m}, 2 \mathrm{H}), 1.63(\mathrm{ddd}, \mathrm{J}=12.6$, $8.2,3.9 \mathrm{~Hz}, 1 \mathrm{H}), 1.14(\mathrm{~d}, \mathrm{~J}=6.8 \mathrm{~Hz}, 3 \mathrm{H}), 1.10(\mathrm{~d}, \mathrm{~J}=7.1 \mathrm{~Hz}, 3 \mathrm{H}), 0.95(\mathrm{~d}, \mathrm{~J}=6.4 \mathrm{~Hz}, 3 \mathrm{H}), 0.92(\mathrm{~d}, \mathrm{~J}=6.4$ $\mathrm{Hz}, 3 \mathrm{H}) .{ }^{13} \mathrm{C}$ NMR (150 MHz, CD $\left.3 \mathrm{OD}\right) \delta 200.4,175.1,173.5,173.4,168.0,166.5,159.7,155.4,143.4$, $135.5,131.9,131.5,129.9,126.1,123.2,119.2$, 114.2, 113.4, 78.7, 75.1, 56.6, 56.3, 44.9, 42.7, 42.0, 40.6, 39.9, 38.9, 38.1, 28.6, 25.8, 23.5, 22.5, 22.0, 17.7, 14.9, 11.5, 11.3. HRMS (ESI) calcd for $\mathrm{C}_{38} \mathrm{H}_{53} \mathrm{ClN}_{4} \mathrm{O}_{9} \mathrm{~S}$ $[\mathrm{M}+\mathrm{H}]$ 777.3295, found 777.3293 .

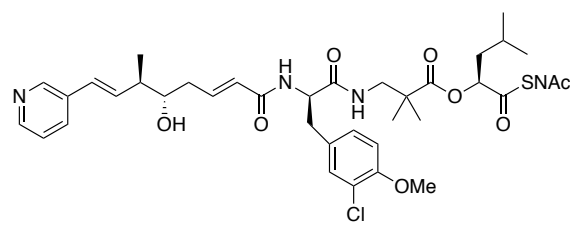

(S)-1-((2-acetamidoethyl)thio)-4-methyl-1-oxopentan-2-yl 3-((R)3-(3-chloro-4-methoxyphenyl)-2-((2E,5S,6R,7E)-5-hydroxy-6methyl-8-(pyridin-3-yl)octa-2,7-dienamido)propanamido)-2,2dimethylpropanoate (15c*). Reaction was run as per general coupling procedure, and purified by flash chromatography system $(1-10 \%$ $\mathrm{MeOH} / \mathrm{DCM}) \mathrm{R}_{\mathrm{f}}=0.65(10 \% \mathrm{MeOH} / \mathrm{DCM}) 10: 1 \mathrm{dr}$. This was then deprotected as per general deprotection 
procedure and purified by flash chromatography system $(2-15 \% \mathrm{MeOH} / \mathrm{DCM})$ to afford the final product 15c* $(0.019 \mathrm{~g}, 51 \%$ yield over 3 steps $)$ as a clear and colorless oil: $R_{\mathrm{f}}=0.25(10 \% \mathrm{MeOH} / \mathrm{DCM}) ;{ }^{1} \mathbf{H}$ NMR $\left(600 \mathrm{MHz}, \mathrm{CD}_{3} \mathrm{OD}\right) \delta 8.52(\mathrm{~d}, \mathrm{~J}=2.3 \mathrm{~Hz}, 1 \mathrm{H}), 8.35(\mathrm{dd}, \mathrm{J}=4.8,1.6 \mathrm{~Hz}, 1 \mathrm{H}), 7.89(\mathrm{dt}, \mathrm{J}=8.0,2.0 \mathrm{~Hz}, 1 \mathrm{H})$, $7.37(\mathrm{dd}, \mathrm{J}=8.0,4.9 \mathrm{~Hz}, 1 \mathrm{H}), 7.28(\mathrm{~d}, \mathrm{~J}=2.2 \mathrm{~Hz}, 1 \mathrm{H}), 7.15(\mathrm{dd}, \mathrm{J}=8.4,2.3 \mathrm{~Hz}, 1 \mathrm{H}), 6.96(\mathrm{~d}, \mathrm{~J}=8.5 \mathrm{~Hz}$, $1 \mathrm{H}), 6.80(\mathrm{dt}, \mathrm{J}=15.0,7.3 \mathrm{~Hz}, 1 \mathrm{H}), 6.46(\mathrm{~d}, \mathrm{~J}=16.1 \mathrm{~Hz}, 1 \mathrm{H}), 6.40(\mathrm{dd}, \mathrm{J}=16.0,7.9 \mathrm{~Hz}, 1 \mathrm{H}), 6.01(\mathrm{~d}, \mathrm{~J}=$ $15.4 \mathrm{~Hz}, 1 \mathrm{H}), 5.20(\mathrm{dd}, \mathrm{J}=9.5,3.8 \mathrm{~Hz}, 1 \mathrm{H}), 4.65(\mathrm{dd}, \mathrm{J}=8.5,6.5 \mathrm{~Hz}, 1 \mathrm{H}), 3.92(\mathrm{p}, \mathrm{J}=6.2 \mathrm{~Hz}, 2 \mathrm{H}), 3.83$ $(\mathrm{s}, 3 \mathrm{H}), 3.66(\mathrm{dt}, \mathrm{J}=8.7,4.6 \mathrm{~Hz}, 1 \mathrm{H}), 3.44-3.36(\mathrm{~m}, 2 \mathrm{H}), 3.33-3.29(\mathrm{~m}, 2 \mathrm{H}), 3.11-3.00(\mathrm{~m}, 3 \mathrm{H}), 2.85$ $(\mathrm{dd}, \mathrm{J}=13.9,8.5 \mathrm{~Hz}, 1 \mathrm{H}), 2.44(\mathrm{td}, \mathrm{J}=7.2,4.3 \mathrm{~Hz}, 1 \mathrm{H}), 2.39(\mathrm{dt}, \mathrm{J}=13.0,6.4 \mathrm{~Hz}, 1 \mathrm{H}), 2.32(\mathrm{dt}, \mathrm{J}=14.9$, $7.6 \mathrm{~Hz}, 1 \mathrm{H}), 1.91(\mathrm{~s}, 3 \mathrm{H}), 1.83-1.69(\mathrm{~m}, 2 \mathrm{H}), 1.64(\mathrm{ddd}, \mathrm{J}=13.4,8.4,3.9 \mathrm{~Hz}, 1 \mathrm{H}), 1.14(\mathrm{~s}, 3 \mathrm{H}) 1.14(\mathrm{~d}$, $3 \mathrm{H}), 1.13(\mathrm{~d}, 3 \mathrm{H}), 0.95(\mathrm{~d}, \mathrm{~J}=6.5 \mathrm{~Hz}, 3 \mathrm{H}), 0.92(\mathrm{~d}, \mathrm{~J}=6.4 \mathrm{~Hz}, 3 \mathrm{H}) .{ }^{13} \mathbf{C} \mathbf{N M R}\left(150 \mathrm{MHz}, \mathrm{CD}_{3} \mathrm{OD}\right) \delta 200.5$, $176.9,173.8,173.4,168.2,155.4,148.3,148.2,143.4,136.4,135.5,134.8,131.9,131.7,129.8,128.0$, $126.2,125.3,123.2,113.4,78.8,75.2,64.7,56.6,56.4,47.9,44.7,44.3,41.9,39.9,38.9,37.9,28.7,25.9$, 25.3, 23.5, 23.3, 23.2, 22.6, 21.9, 17.4. HRMS (ESI) calcd for $\mathrm{C}_{39} \mathrm{H}_{53} \mathrm{ClN}_{4} \mathrm{O}_{8} \mathrm{~S}[\mathrm{M}+\mathrm{H}]$ 773.3345, found 773.3353 .

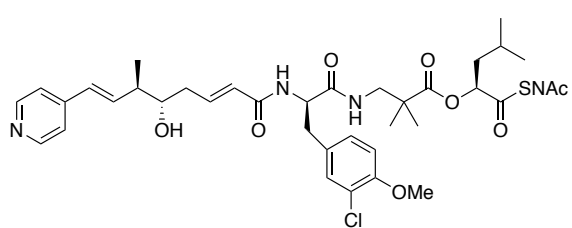

(S)-1-((2-acetamidoethyl)thio)-4-methyl-1-oxopentan-2-yl 3-((R)-3(3-chloro-4-methoxyphenyl)-2-((2E,5S,6R,7E)-5-hydroxy-6methyl-8-(pyridin-4-yl)octa-2,7-dienamido)propanamido)-2,2dimethylpropanoate (15d*). Reaction was run as per general coupling procedure, and purified by flash chromatography system $(1-10 \%$ $\mathrm{MeOH} / \mathrm{DCM}) \mathrm{R}_{\mathrm{f}}=0.65(10 \% \mathrm{MeOH} / \mathrm{DCM}), 12: 1$. This was then deprotected as per general deprotection procedure and purified by flash chromatography system $(2-15 \% \mathrm{MeOH} / \mathrm{DCM})$ to afford the final product 15d* (0.039 g, $45 \%$ yield over 3 steps $)$ as a clear and colorless oil: $\mathrm{R}_{\mathrm{f}}=0.25(10 \% \mathrm{MeOH} / \mathrm{DCM}) ;{ }^{1} \mathbf{H}$ NMR $\left(600 \mathrm{MHz}, \mathrm{CD}_{3} \mathrm{OD}\right) \delta 8.41(\mathrm{~d}, \mathrm{~J}=6.1 \mathrm{~Hz}, 1 \mathrm{H}), 7.40(\mathrm{~d}, \mathrm{~J}=6.1 \mathrm{~Hz}, 1 \mathrm{H}), 7.27(\mathrm{~d}, \mathrm{~J}=1.9 \mathrm{~Hz}, 1 \mathrm{H}), 7.15(\mathrm{dd}$, $\mathrm{J}=8.5,2.2 \mathrm{~Hz}, 1 \mathrm{H}), 6.96(\mathrm{dd}, \mathrm{J}=8.5,1.0 \mathrm{~Hz}, 1 \mathrm{H}), 6.79(\mathrm{dt}, \mathrm{J}=14.5,7.1 \mathrm{~Hz}, 1 \mathrm{H}), 6.61(\mathrm{dd}, \mathrm{J}=16.0,8.5$ $\mathrm{Hz}, 1 \mathrm{H}), 6.43(\mathrm{~d}, \mathrm{~J}=16.0 \mathrm{~Hz}, 1 \mathrm{H}), 6.00(\mathrm{~d}, \mathrm{~J}=15.4 \mathrm{~Hz}, 1 \mathrm{H}), 5.20(\mathrm{dd}, \mathrm{J}=9.5,3.8 \mathrm{~Hz}, 1 \mathrm{H}), 4.65(\mathrm{dd}, \mathrm{J}=$ $8.4,6.6 \mathrm{~Hz}, 1 \mathrm{H}), 3.83(\mathrm{~s}, 3 \mathrm{H}), 3.66(\mathrm{dt}, \mathrm{J}=8.5,4.4 \mathrm{~Hz}, 1 \mathrm{H}), 3.40(\mathrm{~d}, \mathrm{~J}=13.6 \mathrm{~Hz}, 1 \mathrm{H}), 3.36(\mathrm{~d}, \mathrm{~J}=12.9 \mathrm{~Hz}$, $1 \mathrm{H}), 3.32-3.29(\mathrm{~m}, 2 \mathrm{H}), 3.12-2.99(\mathrm{~m}, 3 \mathrm{H}), 2.85(\mathrm{dd}, \mathrm{J}=14.0,8.5 \mathrm{~Hz}, 1 \mathrm{H}), 2.51-2.42(\mathrm{~m}, 1 \mathrm{H}), 2.41-$ $2.34(\mathrm{~m}, 1 \mathrm{H}), 2.35-2.27(\mathrm{~m}, 1 \mathrm{H}), 1.90(\mathrm{~s}, 3 \mathrm{H}), 1.81-1.68(\mathrm{~m}, 2 \mathrm{H}), 1.68-1.60(\mathrm{~m}, 1 \mathrm{H}), 1.16(\mathrm{~s}, 6 \mathrm{H}), 1.15$ $(\mathrm{d}, \mathrm{J}=5.1 \mathrm{~Hz}, 3 \mathrm{H}), 0.95(\mathrm{~d}, \mathrm{~J}=6.5 \mathrm{~Hz}, 3 \mathrm{H}), 0.92(\mathrm{~d}, \mathrm{~J}=6.5 \mathrm{~Hz}, 3 \mathrm{H}) .{ }^{13} \mathbf{C} \mathbf{N M R}\left(150 \mathrm{MHz}, \mathrm{CD}_{3} \mathrm{OD}\right) \delta$ $200.5,176.9,173.7,173.4,168.1,155.4,150.2,147.8,143.2,139.5,131.9,131.7,129.8,129.4,126.2$, 123.2, 122.4, 113.4, 78.8, 75.0, 56.6, 56.3, 47.8, 44.7, 44.3, 41.9, 39.9, 38.9, 37.9, 28.7, 25.9, 23.5, 23.3, 23.2, 22.6, 22.0, 17.2.; HRMS (ESI) calcd for $\mathrm{C}_{39} \mathrm{H}_{53} \mathrm{ClN}_{4} \mathrm{O}_{8} \mathrm{~S}[\mathrm{M}+\mathrm{H}]$ 773.3345, found 773.3353.

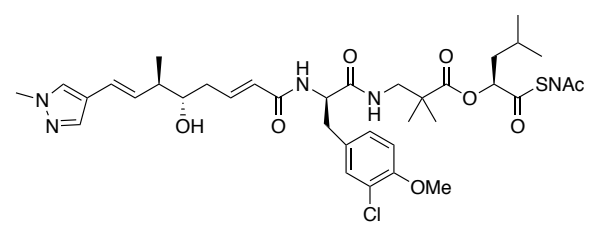

$(S)-1-((2-a c e t a m i d o e t h y l) t h i o)-4-m e t h y l-1-o x o p e n t a n-2-y l ~ 3-((R)-$ 3-(3-chloro-4-methoxyphenyl)-2-((2E,5S,6R,7E)-5-hydroxy-6methyl-8-(1-methyl-1H-pyrazol-4-yl)octa-2,7dienamido)propanamido)-2,2-dimethylpropanoate $(15 h *)$. Reaction was run as per general coupling procedure, and purified by flash chromatography system (1-10\% MeOH/DCM) $\mathrm{R}_{\mathrm{f}}=0.65(10 \% \mathrm{MeOH} / \mathrm{DCM}), 10: 1 \mathrm{dr}$. This was then deprotected as per general deprotection procedure and purified by flash chromatography system $(2-15 \%$ $\mathrm{MeOH} / \mathrm{DCM})$ to afford the final product $\mathbf{1 5 h} *(0.060,41 \%$ yield over 3 steps $)$ as a clear and colorless oil: $\mathrm{R}_{\mathrm{f}}=0.25(10 \% \mathrm{MeOH} / \mathrm{DCM}) ;{ }^{1} \mathbf{H}$ NMR $\left(600 \mathrm{MHz}, \mathrm{CD}_{3} \mathrm{OD}\right) \delta 7.55(\mathrm{~s}, 1 \mathrm{H}), 7.50(\mathrm{~s}, 1 \mathrm{H}), 7.28(\mathrm{~d}, \mathrm{~J}=2.1$ $\mathrm{Hz}, 1 \mathrm{H}), 7.15(\mathrm{dd}, \mathrm{J}=8.5,2.2 \mathrm{~Hz}, 1 \mathrm{H}), 6.96(\mathrm{~d}, \mathrm{~J}=8.5 \mathrm{~Hz}, 1 \mathrm{H}), 6.79(\mathrm{dt}, \mathrm{J}=14.9,7.3 \mathrm{~Hz}, 1 \mathrm{H}), 6.21(\mathrm{~d}, \mathrm{~J}$ $=16.1 \mathrm{~Hz}, 1 \mathrm{H}), 5.99(\mathrm{~d}, \mathrm{~J}=15.4 \mathrm{~Hz}, 1 \mathrm{H}), 5.93(\mathrm{dd}, \mathrm{J}=16.0,8.4 \mathrm{~Hz}, 1 \mathrm{H}), 5.20(\mathrm{dd}, \mathrm{J}=9.6,3.9 \mathrm{~Hz}, 1 \mathrm{H})$, $4.65(\mathrm{dd}, \mathrm{J}=8.6,6.5 \mathrm{~Hz}, 1 \mathrm{H}), 3.84(\mathrm{~s}, 3 \mathrm{H}), 3.83(\mathrm{~s}, 3 \mathrm{H}), 3.60(\mathrm{dt}, \mathrm{J}=8.8,4.6 \mathrm{~Hz}, 1 \mathrm{H}), 3.40(\mathrm{~d}, \mathrm{~J}=13.5 \mathrm{~Hz}$, $1 \mathrm{H}), 3.35(\mathrm{~d}, \mathrm{~J}=13.9 \mathrm{~Hz}, 1 \mathrm{H}), 3.33-3.29(\mathrm{~m}, 2 \mathrm{H}), 3.12-2.98(\mathrm{~m}, 3 \mathrm{H}), 2.85(\mathrm{dd}, \mathrm{J}=13.9,8.6 \mathrm{~Hz}, 1 \mathrm{H})$, $2.32(\mathrm{~m}, 3 \mathrm{H}), 1.91(\mathrm{~s}, 3 \mathrm{H}), 1.83-1.68(\mathrm{~m}, 2 \mathrm{H}), 1.64(\mathrm{ddd}, \mathrm{J}=12.8,8.4,3.8 \mathrm{~Hz}, 1 \mathrm{H}), 1.16(\mathrm{~s}, 6 \mathrm{H}), 1.10(\mathrm{~d}$, $\mathrm{J}=6.9 \mathrm{~Hz}, 3 \mathrm{H}), 0.96(\mathrm{~d}, \mathrm{~J}=6.5 \mathrm{~Hz}, 3 \mathrm{H}), 0.92(\mathrm{~d}, \mathrm{~J}=6.5 \mathrm{~Hz}, 3 \mathrm{H}) .{ }^{13} \mathbf{C} \mathbf{N M R}\left(150 \mathrm{MHz}, \mathrm{CD}_{3} \mathrm{OD}\right) \delta 200.5$, 
$176.8,173.7,173.4,168.2,155.4,143.6,137.7,131.9,131.7,130.9,129.8,129.4,126.0,123.2,122.5$, 121.6, 113.4, 78.8, 75.4, 56.6, 56.4, 47.8, 44.7, 44.1, 41.9, 39.9, 38.7, 38.67, 37.9, 28.7, 25.9, 23.5, 23.2, 23.2, 22.6, 21.9, 17.4. HRMS (ESI) calcd for $\mathrm{C}_{38} \mathrm{H}_{54} \mathrm{ClN}_{5} \mathrm{O}_{8} \mathrm{~S}[\mathrm{M}+\mathrm{H}]$ 776.3454, found 776.3457 .

\section{Biocatalytic Elaboration of Cryptophycin Analogs}

General procedure semi preparative scale reactions

To a $250 \mathrm{~mL}$ Erlenmeyer flask was added DMSO (7\%), and substrate (75 uM) suspended in DMSO. This was diluted with phosphate buffer $(\mathrm{pH}=7.2,100 \mathrm{mM})$ and warmed to $30^{\circ} \mathrm{C}$ for 20 min prior to treatment with CrpTE enzyme $(0.5 \mathrm{uM})$. The reaction was allowed to shake at $100 \mathrm{RPM}$ at $30^{\circ} \mathrm{C}$ for $12 \mathrm{~h}$, prior to $1: 1 \mathrm{v} / \mathrm{v}$ dilution with acetone, and chilling at $-20{ }^{\circ} \mathrm{C}$ in the freezer for $30 \mathrm{~min}$. The precipitated protein was filtered through celite, washed with acetone, organics removed under reduced pressure, and the remaining aqueous layer was extracted with DCM $3 \times 20 \mathrm{~mL}$. The organics were combined, dried over sodium sulfate, filtered, and concentrated. The reactions were purified using a HydroRP C18 (250 x 10.0 $\mathrm{mm}, 4$ micron): $20-80 \%$ water/acetonitrile gradient with a flow rate of $3 \mathrm{~mL} / \mathrm{min}$.

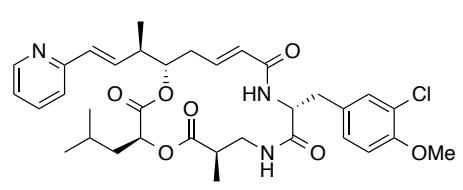

(3S,6R,10R,16S,E)-10-(3-chloro-4-methoxybenzyl)-3-isobutyl-6-methyl16-((R,E)-4-(pyridin-2-yl)but-3-en-2-yl)-1,4-dioxa-8,11diazacyclohexadec-13-ene-2,5,9,12-tetraone (16b). Reaction was run and purified as general procedure for semi-preparative scale reaction. Retention time: 36 minutes, ${ }^{\mathbf{1}} \mathbf{H}$ NMR $\left(600 \mathrm{MHz}, \mathrm{CD}_{3} \mathrm{OD}\right) \delta 8.46(\mathrm{dd}, \mathrm{J}=5.0,0.9 \mathrm{~Hz}, 1 \mathrm{H}, \mathrm{NCHCH}$ unit A Ar), 7.78 (td, J = 7.7, $1.8 \mathrm{~Hz}, 1 \mathrm{H}, \mathrm{CHCHCHC}$ unit A Ar), 7.48 (d, J = 7.9 Hz, 1H, CHCHC unit A Ar), 7.28 (d, J = $2.2 \mathrm{~Hz}, 1 \mathrm{H}, \mathrm{CCHCCl}$ unit B), 7.26 (ddd, J = 7.5, 5.0, 1.1 Hz, 1H, NCHCHCH unit A Ar), 7.17 (dd, J = 8.5, $2.2 \mathrm{~Hz}, 1 \mathrm{H}, \mathrm{CCHCH}$ unit B), 6.98 (d, J = 8.5 Hz, 1H, CCHCHC unit B), 6.71 (ddd, J = 15.1, 11.1, 3.9 Hz, $1 \mathrm{H}, \mathrm{CH}_{2} \mathrm{CH}=\mathrm{CH}$ unit A), $6.61-6.57(\mathrm{~m}, 2 \mathrm{H}, \mathrm{CCH}=\mathrm{CHCH}$ unit A), $5.93(\mathrm{dd}, \mathrm{J}=15.1,1.9 \mathrm{~Hz}, 1 \mathrm{H}$, $\mathrm{CH}=\mathrm{CHCO}$ unit A), 5.09 (ddd, $\mathrm{J}=11.3,6.9,1.9 \mathrm{~Hz}, 1 \mathrm{H}, \mathrm{CHCHCH}_{2}$ unit A), 4.93 (dd, $\mathrm{J}=9.9,3.6 \mathrm{~Hz}, 1 \mathrm{H}$, COCHO unit D), 4.52 (dd, $\mathrm{J}=11.3,3.9 \mathrm{~Hz}, 1 \mathrm{H}, \mathrm{COCHNH}$ Unit B), 3.84 (s, 3H, COCH $\boldsymbol{H}_{3}$ unit B), 3.58 (dd, $\mathrm{J}=13.8,3.3 \mathrm{~Hz}, 1 \mathrm{H}, 1 \times \mathrm{CHCH}_{2} \mathrm{NH}$ unit $\left.\mathrm{C}\right), 3.27\left(\mathrm{dd}, \mathrm{J}=13.8,3.0 \mathrm{~Hz}, 1 \mathrm{H}, 1 \mathrm{x} \mathrm{CHCH}_{2} \mathrm{NH}\right.$ unit C), 3.18 (dd, $\mathrm{J}=14.5,3.9 \mathrm{~Hz}, 1 \mathrm{H}, 1 \times \mathrm{CHCH}_{2} \mathrm{C}$ unit B), 2.80-2.65 (m, 4H, 1x $\mathrm{CHCH}_{2} \mathrm{C}$ unit B, $\mathrm{COCHCH}_{2}$ unit $\mathrm{C}$, $\mathrm{CHCHCHO}$ unit A \& $1 \times \mathrm{CHOCCH}_{2} \mathrm{CH}$ unit A), $2.39\left(\mathrm{dt}, \mathrm{J}=14.5,11.2 \mathrm{~Hz}, 1 \mathrm{H}, 1 \mathrm{x} \mathrm{CHOCCH}_{2} \mathrm{CH}\right.$ unit A), $1.69-1.52\left(\mathrm{~m}, 2 \mathrm{H},\left(\mathrm{CH}_{3}\right)_{2} \mathrm{CHCH}_{2}\right.$ unit D \& 1x $\mathrm{CHCH}_{2} \mathrm{CH}$ unit D), 1.34 (ddd, $\mathrm{J}=14.1,8.8,3.6 \mathrm{~Hz}, 1 \mathrm{H}$, $\mathrm{CHCH}_{2} \mathrm{CH}$ unit D), $1.18\left(\mathrm{~d}, \mathrm{~J}=7.1 \mathrm{~Hz}, 6 \mathrm{H}, \mathrm{CH}_{3} \mathrm{CH}\right.$ unit $\mathrm{A} \& \mathrm{CH}_{3} \mathrm{CH}$ unit $\left.\mathrm{C}\right), 0.74(\mathrm{~d}, \mathrm{~J}=6.5 \mathrm{~Hz}, 3 \mathrm{H}, 1 \mathrm{x}$ $\left(\mathrm{CH}_{3}\right)_{2} \mathrm{CH}$ unit D), $0.71\left(\mathrm{~d}, \mathrm{~J}=6.6 \mathrm{~Hz}, 3 \mathrm{H}, 1 \mathrm{x}\left(\mathrm{CH}_{3}\right)_{2} \mathrm{CH}\right.$ unit D). ${ }^{13} \mathbf{C} \mathbf{N M R}\left(150 \mathrm{MHz}, \mathrm{CD}_{3} \mathrm{OD}\right) \delta 177.5$ (OCOCH unit $\mathrm{C}$ ), 174.0 ( $\mathrm{NHCOCH}$ unit $\mathrm{B}), 172.2$ (OCOCH unit D), 168.3 (CHCONH unit A), 156.6 $(\mathrm{NCHCH}$ unit $\mathrm{A} \mathrm{Ar}), 155.4(\mathrm{CHCHCC}$ unit $\mathrm{B}), 150.0\left(\mathrm{NCCH}\right.$ unit A Ar), $143.4\left(\mathrm{CH}_{2} \mathrm{CH}=\mathrm{CH}\right.$ unit $\left.\mathrm{A}\right)$, 138.7 ( $\mathrm{NCCHCH}$ unit A Ar), 137.6 (CHCHCHC unit A Ar), 132.2 (NCHCHCH unit A Ar), 132.1 $\left(\mathrm{CH}_{2} \mathrm{CCH}_{2}\right.$ unit $\left.\mathrm{B}\right), 131.5$ (CCHC unit B), $129.3(\mathrm{CCHCH}$ unit B), 125.6 (CHCHCO unit A), 123.71 ( $\mathrm{CHCHC}$ unit A Ar), $123.3(\mathrm{CHCCl}$ Unit B), $122.8(\mathrm{CCH}=\mathrm{CHCH}), 113.5(\mathrm{CCHCHC}$ unit B), 78.5 $\left(\mathrm{CHCHCH}_{2}\right.$ unit A), 72.9 ( $\mathrm{COCHO}$ unit D), $57.4\left(\mathrm{COCHNH}\right.$ unit B), $56.6\left(\mathrm{CHOCH}_{3}\right.$ unit B), 43.5 $\left(\mathrm{CHCHCHCH}_{2}\right.$ unit A), $41.2\left(\mathrm{CHCH}_{2} \mathrm{NH}\right.$ unit $\left.\mathrm{C}\right), 40.9\left(\mathrm{CHCH}_{2} \mathrm{CH}\right.$ unit D), $39.0\left(\mathrm{COCHCH}_{2}\right.$ unit C), 38.0 $\left(\mathrm{CHCH}_{2} \mathrm{CH}\right.$ unit A), $36.4\left(\mathrm{CHCH}_{2} \mathrm{C}\right.$ unit B), $25.6\left(\left(\mathrm{CH}_{3}\right)_{2} \mathrm{CHCH}_{2}\right.$ unit D), $23.2\left(1 \mathrm{x}\left(\mathrm{CH}_{3}\right)_{2} \mathrm{CH}\right.$ unit D), 21.6 $\left(1 \mathrm{x}\left(\boldsymbol{C H}_{3}\right)_{2} \mathrm{CH}\right.$ unit D), $17.5\left(1 \mathrm{x} \mathrm{CH}_{3} \mathrm{CH}\right.$ unit A), 15.1 (1x $\mathrm{CHCH}_{3}$ unit C). HRMS (ESI) calcd for $\mathrm{C}_{34} \mathrm{H}_{42} \mathrm{ClN}_{3} \mathrm{O}_{7}[\mathrm{M}+\mathrm{H}] 640.2784$, found 640.2788 .

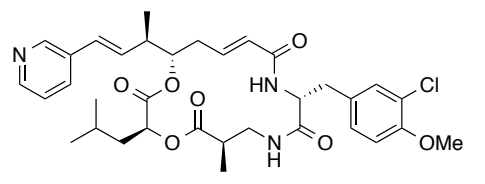

(3S,6R,10R,16S,E)-10-(3-chloro-4-methoxybenzyl)-3-isobutyl-6-methyl16-((R,E)-4-(pyridin-3-yl)but-3-en-2-yl)-1,4-dioxa-8,11diazacyclohexadec-13-ene-2,5,9,12-tetraone (16c). Reaction was run and purified as general procedure for semi-preparative scale reaction. Retention time: 39 minutes, ${ }^{1} \mathbf{H}$ NMR $\left(600 \mathrm{MHz}, \mathrm{CD}_{3} \mathrm{OD}\right) \delta 8.53(\mathrm{~d}, \mathrm{~J}=2.2 \mathrm{~Hz}, 1 \mathrm{H}, \mathrm{NCHC}$ unit A), $8.38(\mathrm{dd}, \mathrm{J}=4.8$, 
$1.6 \mathrm{~Hz}, 1 \mathrm{H}, \mathrm{NCHCH}$ unit A), $7.92(\mathrm{~d}, \mathrm{~J}=8.0 \mathrm{~Hz}, 1 \mathrm{H}, \mathrm{NCHCHCH}$ unit A), 7.40 (dd, J = 8.0, 4.9 Hz, 1H, CHCHC unit A), 7.28 (d, J = 2.2 Hz, 1H, CCHCCl unit B), 7.17 (dd, J = 8.4, 2.2 Hz, 1H, CCHCH unit B), $6.98\left(\mathrm{~d}, \mathrm{~J}=8.4 \mathrm{~Hz}, 1 \mathrm{H}, \mathrm{CCHCHC}\right.$ unit B), 6.71 (ddd, $\mathrm{J}=15.1,11.2,3.9 \mathrm{~Hz}, 1 \mathrm{H}, \mathrm{CH}_{2} \mathrm{CH}=\mathrm{CH}$ unit A), 6.53 $(\mathrm{d}, \mathrm{J}=15.9 \mathrm{~Hz}, 1 \mathrm{H}, \mathrm{CC} \boldsymbol{H}=\mathrm{CH}$ unit A), $6.30(\mathrm{dd}, \mathrm{J}=15.9,8.9 \mathrm{~Hz}, 1 \mathrm{H}, \mathrm{CCH}=\mathrm{CHCH}$ unit A), 5.93 (dd, $\mathrm{J}=$ 15.2, $1.9 \mathrm{~Hz}, 1 \mathrm{H}, \mathrm{CH}=\mathrm{CHCO}$ unit A), 5.08 (ddd, $\mathrm{J}=11.2,7.2,1.9 \mathrm{~Hz}, 1 \mathrm{H}, \mathrm{CHCHCH}_{2}$ unit A), 4.93 (dd, J $=9.9,3.7 \mathrm{~Hz}, 1 \mathrm{H}, \mathrm{COCHO}$ unit D), $4.52\left(\mathrm{dd}, \mathrm{J}=11.2,3.9 \mathrm{~Hz}, 1 \mathrm{H}, \mathrm{COCHNH}\right.$ Unit B), $3.84\left(\mathrm{~s}, 3 \mathrm{H}, \mathrm{COCH}_{3}\right.$ unit B), 3.58 (dd, $\mathrm{J}=13.8,3.3 \mathrm{~Hz}, 1 \mathrm{H}, 1 \times \mathrm{CHCH}_{2} \mathrm{NH}$ unit C), 3.27 (dd, $\mathrm{J}=13.8,3.0 \mathrm{~Hz}, 1 \mathrm{H}, 1 \times \mathrm{CHCH}_{2} \mathrm{NH}$ unit C), $3.18\left(\mathrm{dd}, \mathrm{J}=14.5,3.9 \mathrm{~Hz}, 1 \mathrm{H}, 1 \mathrm{x} \mathrm{CHCH}_{2} \mathrm{C}\right.$ unit B), $2.77-2.72\left(\mathrm{~m}, 1 \mathrm{H}, \mathrm{COCHCH}_{2}\right.$ unit $\left.\mathrm{C}\right) 2.73$ $\left(\mathrm{dd}, \mathrm{J}=14.5,11.5,1 \mathrm{H}, 1 \times \mathrm{CHCH}_{2} \mathrm{C}\right.$ unit B) $2.70-2.61\left(\mathrm{~m}, 2 \mathrm{H}, \mathrm{CHCHCHO}\right.$ unit A \& $1 \mathrm{x} \mathrm{CHOCCH} \boldsymbol{H}_{2} \mathrm{CH}$ unit A), 2.37 (dt, J = 14.4, $11.1 \mathrm{~Hz}, 1 \mathrm{H}, 1 \times \mathrm{CHOCCH}_{2} \mathrm{CH}$ unit A), $1.69-1.55\left(\mathrm{~m}, 2 \mathrm{H},\left(\mathrm{CH}_{3}\right)_{2} \mathrm{CHCH}_{2}\right.$ unit $\mathrm{D} \& 1 \mathrm{x} \mathrm{CHCH}_{2} \mathrm{CH}$ unit D), $1.31\left(\mathrm{ddd}, \mathrm{J}=14.1,8.9,3.7 \mathrm{~Hz}, 1 \mathrm{H}, \mathrm{CHCH}{ }_{2} \mathrm{CH}\right.$ unit D), $1.19(\mathrm{~d}, \mathrm{~J}=3.5 \mathrm{~Hz}$, $3 \mathrm{H}, \mathrm{CH}_{3} \mathrm{CH}$ unit A), $1.18\left(\mathrm{~d}, \mathrm{~J}=2.9 \mathrm{~Hz}, 3 \mathrm{H}, \mathrm{CH}_{3} \mathrm{CH}\right.$ unit $\left.\mathrm{C}\right), 0.74\left(\mathrm{~d}, \mathrm{~J}=6.5 \mathrm{~Hz}, 3 \mathrm{H}, 1 \mathrm{x}\left(\mathrm{CH}_{3}\right)_{2} \mathrm{CH}\right.$ unit D), $0.70\left(\mathrm{~d}, \mathrm{~J}=6.5 \mathrm{~Hz}, 3 \mathrm{H}, 1 \mathrm{x}\left(\mathrm{CH}_{3}\right)_{2} \mathrm{CH}\right.$ unit D). ${ }^{13} \mathbf{C} \mathbf{~ N M R}\left(150 \mathrm{MHz}, \mathrm{CD}_{3} \mathrm{OD}\right) \delta 177.5(\mathrm{OCOCH}$ unit C), 174.0 (NHCOCH unit $\mathrm{B}), 172.2$ (OCOCH unit D), 168.3 (CHCONH unit A), 155.4 (CHCHCC unit B), 148.7 ( $\mathrm{NCHC}$ unit A Ar), $148.4(\mathrm{NCHCH}$ unit $\mathrm{A} \mathrm{Ar}), 143.4\left(\mathrm{CH}_{2} \boldsymbol{C H}=\mathrm{CH}\right.$ unit A), $135.6(\mathrm{CCH}=\boldsymbol{C H C H}$ unit A), 135.0 ( $\mathrm{CHCHC}$ unit A Ar), 134.9 ( $\mathrm{CHCCH}$ unit A Ar), $132.2\left(\mathrm{CH}_{2} \mathrm{CCH}_{2}\right.$ unit B), $131.5 \mathrm{CC} \mathrm{HC}$ unit $\mathrm{B}), 129.3$ (CCHCH unit $\mathrm{B}), 128.8$ ( $\mathrm{NCHCHCH}$ unit $\mathrm{A} \mathrm{Ar}), 125.6(\mathrm{CCH}=\mathrm{CH}$ unit A), $125.4(\mathrm{CHCHCO}$ unit A), 113.5 (CCHCHC unit B), $78.5\left(\mathrm{CHCHCH}_{2}\right.$ unit A), 72.8 (COCHO unit D), $57.4(\mathrm{COCHNH}$ unit B), $56.6\left(\mathrm{CHOCH}_{3}\right.$ unit B), $43.7\left(\mathrm{CHCH}_{2} \mathrm{~N}\right.$ unit $\left.\mathrm{C}\right), 41.2\left(\mathrm{CCH}_{2}\right.$ unit $\left.\mathrm{C}\right), 40.9\left(\mathrm{CHCH}_{2} \mathrm{CH}\right.$ unit D), 39.0 $\left(\mathrm{COCHCH}_{2}\right.$ unit $\left.\mathrm{C}\right), 37.9\left(\mathrm{CHCH}_{2} \mathrm{CH}\right.$ unit A), $36.4\left(\mathrm{CHCH}_{2} \mathrm{C}\right.$ unit B), $25.6\left(\left(\mathrm{CH}_{3}\right)_{2} \mathrm{CHCH}_{2}\right.$ unit D), 23.2 (1x $\left(\boldsymbol{C H}_{3}\right)_{2} \mathrm{CH}$ unit D), $21.6\left(1 \mathrm{x}\left(\mathrm{CH}_{3}\right)_{2} \mathrm{CH}\right.$ unit D), 17.5 (1x $\boldsymbol{C H}_{3} \mathrm{CH}$ unit A), 15.1 (1x $\mathrm{CHCH}_{3}$ unit $\mathrm{C}$ ). HRMS (ESI) calcd for $\mathrm{C}_{34} \mathrm{H}_{42} \mathrm{ClN}_{3} \mathrm{O}_{7}[\mathrm{M}+\mathrm{H}] 640.2784$, found 640.2789.

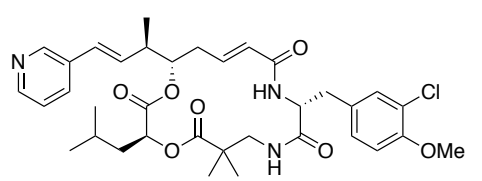

(3S,10R,16S,E)-10-(3-chloro-4-methoxybenzyl)-3-isobutyl-6,6dimethyl-16-((R,E)-4-(pyridin-3-yl)but-3-en-2-yl)-1,4-dioxa-8,11diazacyclohexadec-13-ene-2,5,9,12-tetraone (16c*). Reaction was run and purified as general procedure for semi-preparative scale reaction. Retention time: 43 minutes, ${ }^{1} \mathbf{H}$ NMR $\left(600 \mathrm{MHz}, \mathrm{CD}_{3} \mathrm{OD}\right) \delta 8.53(\mathrm{~d}, \mathrm{~J}=2.2 \mathrm{~Hz}, 1 \mathrm{H}, \mathrm{NCHC}$ unit A), 8.38 (dd, J $=4.8$, $1.6 \mathrm{~Hz}, 1 \mathrm{H}, \mathrm{NCHCH}$ unit A), 7.92 (dt, J = 8.0, 1.9 Hz, 1H, NCHCHCH unit A), 7.40 (dd, J = 8.0, 4.8 Hz, 1H, CHCHC unit A), 7.28 (d, J = 2.2 Hz, 1H, CCHCCl unit B), 7.17 (dd, J = 8.4, 2.2 Hz, 1H, CCHCH unit B), $6.98\left(\mathrm{~d}, \mathrm{~J}=8.4 \mathrm{~Hz}, 1 \mathrm{H}, \mathrm{CCHCHC}\right.$ unit B), $6.72\left(\mathrm{ddd}, \mathrm{J}=15.2,11.2,3.9 \mathrm{~Hz}, 1 \mathrm{H}, \mathrm{CH}_{2} \mathrm{CH}=\mathrm{CH}\right.$ unit A), $6.53(\mathrm{~d}, \mathrm{~J}=15.9 \mathrm{~Hz}, 1 \mathrm{H}, \mathrm{CCH}=\mathrm{CH}$ unit A), $6.30(\mathrm{dd}, \mathrm{J}=16.0,8.9 \mathrm{~Hz}, 1 \mathrm{H}, \mathrm{CCH}=\mathrm{CHCH}$ unit A), 5.92 (dd, $\mathrm{J}=15.1,1.9 \mathrm{~Hz}, 1 \mathrm{H}, \mathrm{CH}=\mathrm{CHCO}$ unit A), 5.07 (ddd, $\mathrm{J}=11.1,8.3 \mathrm{~Hz}, 1 \mathrm{H}, \mathrm{CHCHCH}_{2}$ unit A), 4.96 (dd, $\mathrm{J}$ $=9.9,3.4 \mathrm{~Hz}, 1 \mathrm{H}, \mathrm{COCHO}$ unit D), 4.51 (dd, J = 11.3, $3.8 \mathrm{~Hz}, 1 \mathrm{H}, \mathrm{COCHNH}$ Unit B), $3.84\left(\mathrm{~s}, 3 \mathrm{H}, \mathrm{COCH}_{3}\right.$ unit B), $3.46\left(\mathrm{~d}, \mathrm{~J}=13.6 \mathrm{~Hz}, 1 \mathrm{H}, 1 \times \mathrm{CCH}_{2} \mathrm{NH}\right.$ unit $\left.\mathrm{C}\right), 3.18\left(\mathrm{dd}, \mathrm{J}=14.5,3.8 \mathrm{~Hz}, 1 \mathrm{H}, 1 \times \mathrm{CHCH}_{2} \mathrm{C}\right.$ unit B), $3.08\left(\mathrm{~d}, \mathrm{~J}=13.7 \mathrm{~Hz}, 1 \mathrm{H}, 1 \times \mathrm{CCH}_{2} \mathrm{NH}\right.$ unit $\left.\mathrm{C}\right), 2.74\left(\mathrm{dd}, \mathrm{J}=14.5,11.4 \mathrm{~Hz}, 1 \mathrm{H}, 1 \mathrm{x} \mathrm{CHCH}_{2} \mathrm{C}\right.$ unit B), 2.71 - $2.62\left(\mathrm{~m}, 2 \mathrm{H}, \mathrm{CHCHCHO}\right.$ unit A \& 1x $\mathrm{CHOCCH}_{2} \mathrm{CH}$ unit A), $2.36(\mathrm{dt}, \mathrm{J}=14.4,11.2 \mathrm{~Hz}, 1 \mathrm{H}, 1 \mathrm{x}$ $\mathrm{CHOCCH}_{2} \mathrm{CH}$ unit A), $1.66-1.55\left(\mathrm{~m}, 2 \mathrm{H},\left(\mathrm{CH}_{3}\right)_{2} \mathrm{CHCH}_{2}\right.$ unit D \& $1 \mathrm{x} \mathrm{CHCH}_{2} \mathrm{CH}$ unit D), $1.37-1.26$ (m, $1 \mathrm{H}, 1 \times \mathrm{CHCH}_{2} \mathrm{CH}$ unit D), 1.20 (s, 3H, 1 x(CH $\mathrm{CH}_{2} \mathrm{C}$ unit C), 1.18 (d, J =6.9 Hz, 3H, $\mathrm{CH}_{3} \mathrm{CH}$ unit A), 1.16 $\left(\mathrm{s}, 3 \mathrm{H}, 1 \times\left(\mathrm{CH}_{3}\right)_{2} \mathrm{C}\right), 0.74\left(\mathrm{~d}, \mathrm{~J}=6.4 \mathrm{~Hz}, 3 \mathrm{H}, 1 \times\left(\mathrm{CH}_{3}\right)_{2} \mathrm{CH}\right.$ unit D), $0.70\left(\mathrm{~d}, \mathrm{~J}=6.4 \mathrm{~Hz}, 3 \mathrm{H}, 1 \times(\mathrm{CH})_{2} \mathrm{CH}\right.$ unit D). ${ }^{13} \mathbf{C}$ NMR $\left(150 \mathrm{MHz}, \mathrm{CD}_{3} \mathrm{OD}\right) \delta 178.9(\mathrm{OCOCH}$ unit C), 173.7 (NHCOCH unit B), $172.0(\mathrm{OCOCH}$ unit D), 168.2 (CHCONH unit A), 155.4 (CHCHCC unit B), 148.7 (NCHC unit A Ar), 148.4 (NCHCH unit A Ar), $143.6\left(\mathrm{CH}_{2} \mathrm{CH}=\mathrm{CH}\right.$ unit A), $135.5(\mathrm{CCH}=\boldsymbol{C H C H}$ unit A), $135.0(\mathrm{CHCHC}$ unit A Ar), 134.8 ( $\mathrm{CHCCH}$ unit A Ar), $132.2\left(\mathrm{CH}_{2} \mathrm{CCH}_{2}\right.$ unit B), 131.5 (CCHC unit B), 129.3 (CCHCH unit B), 128.8 ( $\mathrm{NCHCHCH}$ unit $\mathrm{A} \mathrm{Ar}), 125.4(\mathrm{CCH}=\mathrm{CH}$ unit $\mathrm{A}), 125.4$ (CHCHCO unit $\mathrm{A}), 123.3$ (CHCCl Unit B), 113.5 (CCHCHC unit B), $78.4\left(\mathrm{CHCHCH}_{2}\right.$ unit $\left.\mathrm{A}\right), 72.6$ (COCHO unit D), 57.5 (COCHNH unit B), 56.6 $\left(\mathrm{CHOCH}_{3}\right.$ unit $\left.\mathrm{B}\right), 47.4\left(\mathrm{CCH}_{2} \mathrm{~N}\right.$ unit $\left.\mathrm{C}\right), 44.0\left(\mathrm{COCCH}_{2}\right.$ unit $\left.\mathrm{C}\right), 43.7\left(\mathrm{CHCHCHCH}_{2}\right.$ unit $\left.\mathrm{A}\right), 41.2$ $\left(\mathrm{CHCH}_{2} \mathrm{NH}\right.$ unit C), $40.9\left(\mathrm{CHCH}_{2} \mathrm{CH}\right.$ unit D), $37.9\left(\mathrm{CHCH}_{2} \mathrm{CH}\right.$ unit $\left.\mathrm{A}\right), 36.5\left(\mathrm{CHCH}_{2} \mathrm{C}\right.$ unit B), 25.8 $\left(\left(\mathrm{CH}_{3}\right)_{2} \boldsymbol{C} \mathrm{HCH}_{2} \text { unit D), } 23.3 \text { (1x C( } \boldsymbol{C} \mathrm{H}_{3}\right)_{2}$ unit C), 23.3 (1x C( $\left.\boldsymbol{C H}_{3}\right)_{2}$ unit C), $23.2\left(1 \times\left(\boldsymbol{C} \mathrm{H}_{3}\right)_{2} \mathrm{CH}\right.$ unit D), 
$21.6\left(1 \mathrm{x}\left(\mathrm{CH}_{3}\right)_{2} \mathrm{CH}\right.$ unit D), 17.5 (1x $\mathrm{CH}_{3} \mathrm{CH}$ unit A). HRMS (ESI) calcd for $\mathrm{C}_{35} \mathrm{H}_{44} \mathrm{ClN}_{3} \mathrm{O}_{7}[\mathrm{M}+\mathrm{H}]$ 654.2941, found 654.2940.

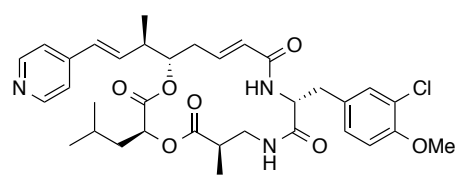

(3S,6R,10R,16S,E)-10-(3-chloro-4-methoxybenzyl)-3-isobutyl-6-methyl16-((R,E)-4-(pyridin-4-yl)but-3-en-2-yl)-1,4-dioxa-8,11diazacyclohexadec-13-ene-2,5,9,12-tetraone (16d). Reaction was run and purified as general procedure for semi-preparative scale reaction. Retention time: 33 minutes, ${ }^{1} \mathbf{H}$ NMR $\left(600 \mathrm{MHz}, \mathrm{CD}_{3} \mathrm{OD}\right) \delta 8.43(\mathrm{~d}, \mathrm{~J}=6.2 \mathrm{~Hz}, 2 \mathrm{H}, 2 \mathrm{x}$ NCHCH unit A Ar), $7.42(\mathrm{~d}$, $\mathrm{J}=6.3 \mathrm{~Hz}, 2 \mathrm{H}, 2 \mathrm{x}$ CHCHC unit A Ar), $7.27(\mathrm{~d}, \mathrm{~J}=2.1 \mathrm{~Hz}, 1 \mathrm{H}, \mathrm{CCHCCl}$ unit B), 7.15 (dd, J = 8.5, 2.1 Hz, $1 \mathrm{H}, \mathrm{CCHCH}$ unit B), $6.96(\mathrm{~d}, \mathrm{~J}=8.4 \mathrm{~Hz}, 1 \mathrm{H}, \mathrm{CCHCHC}$ unit B), 6.69 (ddd, J = 15.1, 11.1, 3.9 Hz, 1H, $\mathrm{CH}_{2} \mathrm{CH}=\mathrm{CH}$ unit A), $6.50-6.47(\mathrm{~m}, 2 \mathrm{H}, \mathrm{CC} \boldsymbol{H}=\mathrm{C} \boldsymbol{H C H}$ unit A), $5.91(\mathrm{dd}, \mathrm{J}=15.2,1.8 \mathrm{~Hz}, 1 \mathrm{H}, \mathrm{CH}=\mathrm{C} \boldsymbol{H C O}$ unit A), 5.08 (ddd, $\mathrm{J}=10.7,8.3 \mathrm{~Hz}, 1 \mathrm{H}, \mathrm{CHCHCH}_{2}$ unit A), $4.90(\mathrm{dd}, \mathrm{J}=9.9,3.6 \mathrm{~Hz}, 1 \mathrm{H}, \mathrm{COCHO}$ unit D), $4.51\left(\mathrm{dd}, \mathrm{J}=11.2,3.9 \mathrm{~Hz}, 1 \mathrm{H}, \mathrm{COCHNH}\right.$ Unit B), 3.82 (s, 3H, COCH $\boldsymbol{H}_{3}$ unit B), 3.56 (dd, $\mathrm{J}=13.8,3.3$ $\mathrm{Hz}, 1 \mathrm{H}, 1 \mathrm{x} \mathrm{CHCH}_{2} \mathrm{NH}$ unit C), 3.25 (dd, $\mathrm{J}=13.8,2.9 \mathrm{~Hz}, 1 \mathrm{H}, 1 \mathrm{x} \mathrm{CHCH}_{2} \mathrm{NH}$ unit C), 3.16 (dd, J = 14.5, $3.9 \mathrm{~Hz}, 1 \mathrm{H}, 1 \times \mathrm{CHCH}_{2} \mathrm{C}$ unit B), $2.77-2.62\left(\mathrm{~m}, 4 \mathrm{H}, \mathrm{COCHCH}_{2}\right.$ unit $\mathrm{C}, 1 \times \mathrm{CHCH}_{2} \mathrm{C}$ unit B, CHCHCHO unit A \& 1x CHOCCH$H_{2} \mathrm{CH}$ unit A), $2.34\left(\mathrm{dt}, \mathrm{J}=14.7,11.2 \mathrm{~Hz}, 1 \mathrm{H}, 1 \times \mathrm{CHOCCH}{ }_{2} \mathrm{CH}\right.$ unit A), $1.67-1.51$ $\left(\mathrm{m}, 2 \mathrm{H}, 1 \mathrm{x} \mathrm{CHCH}_{2} \mathrm{CH}\right.$ unit D \& $\left(\mathrm{CH}_{3}\right)_{2} \mathrm{CHCH}_{2}$ unit D), 1.28 (ddd, $\mathrm{J}=12.9,8.7,3.5 \mathrm{~Hz}, 1 \mathrm{H}, 1 \mathrm{x} \mathrm{CHCH}_{2} \mathrm{CH}$ unit D), $1.17\left(\mathrm{~d}, \mathrm{~J}=2.6 \mathrm{~Hz}, 3 \mathrm{H}, \mathrm{CH}_{3} \mathrm{CH}\right.$ unit $\left.\mathrm{C}\right), 1.16\left(\mathrm{~d}, \mathrm{~J}=2.0 \mathrm{~Hz}, 3 \mathrm{H}, \mathrm{CH} \mathrm{H}_{3} \mathrm{CH}\right.$ unit A), $0.72(\mathrm{~d}, \mathrm{~J}=6.4$ $\mathrm{Hz}, 3 \mathrm{H}, 1 \times\left(\mathrm{CH}_{3}\right)_{2} \mathrm{CH}$ unit D), $0.68\left(\mathrm{~d}, \mathrm{~J}=6.5 \mathrm{~Hz}, 3 \mathrm{H}, 1 \times\left(\mathrm{CH}_{3}\right)_{2} \mathrm{CH}\right.$ unit D). ${ }^{13} \mathbf{C ~ N M R}\left(150 \mathrm{MHz}, \mathrm{CD}_{3} \mathrm{OD}\right)$ $\delta 177.6(\mathrm{OCOCH}$ unit $\mathrm{C}), 174.0(\mathrm{NHCOCH}$ unit $\mathrm{B}), 172.2(\mathrm{OCOCH}$ unit $\mathrm{D}), 168.3(\mathrm{CHCONH}$ unit $\mathrm{A})$, 155.4 (CHCHCC unit B), $150.4\left(\mathrm{NCHCH}\right.$ unit A Ar), $147.1\left(\mathrm{CHCCH}\right.$ unit A Ar), $143.3\left(\mathrm{CH}_{2} \mathrm{CH}=\mathrm{CH}\right.$ unit A), $138.6\left(\mathrm{CH}=\boldsymbol{C H C H}\right.$ unit A), $132.2\left(\mathrm{CH}_{2} \boldsymbol{C C H}_{2}\right.$ unit B), $131.5(\mathrm{C} \boldsymbol{C H C}$ unit $\mathrm{B}), 130.2(\mathrm{CCH}=\mathrm{CH}$ unit $\mathrm{A})$, $129.3(\mathrm{CCHCH}$ unit $\mathrm{B}), 125.7$ ( $\mathrm{CHCHCO}$ unit $\mathrm{A}), 123.3(\mathrm{CHCCl}$ Unit $\mathrm{B}), 122.5$ ( $\mathrm{NCHCHC}$ unit A Ar), 113.5 (CCHCHC unit B), $78.4\left(\mathrm{CHCHCH}_{2}\right.$ unit A), 72.8 (COCHO unit D), 57.4 (COCHNH unit B), 56.6 $\left(\mathrm{CHOCH}_{3}\right.$ unit B), $43.7\left(\mathrm{CHCHCHCH}_{2}\right.$ unit $\left.\mathrm{A}\right), 41.2\left(\mathrm{CHCH}_{2} \mathrm{NH}\right.$ unit $\left.\mathrm{C}\right), 40.9\left(\mathrm{CHCH}_{2} \mathrm{CH}\right.$ unit D), 39.0 $\left(\mathrm{COCHCH}_{2}\right.$ unit $\left.\mathrm{C}\right), 37.9\left(\mathrm{CHCH}_{2} \mathrm{CH}\right.$ unit A), $36.4\left(\mathrm{CHCH}_{2} \mathrm{C}\right.$ unit B), $25.6\left(\left(\mathrm{CH}_{3}\right)_{2} \mathrm{CHCH}_{2}\right.$ unit D), 23.2 (1x $\left.\left(\boldsymbol{C H}_{3}\right)_{2} \mathrm{CH}\right), 21.7\left(1 \mathrm{x}\left(\boldsymbol{C} \mathrm{H}_{3}\right)_{2} \mathrm{CH}\right), 17.4\left(1 \mathrm{x} \boldsymbol{C} \mathrm{H}_{3} \mathrm{CH}\right.$ unit A), 15.1 (1x $\mathrm{CH}_{\boldsymbol{C}} \mathrm{H}_{3}$ unit $\left.\mathrm{C}\right)$. HRMS (ESI) calcd for $\mathrm{C}_{34} \mathrm{H}_{42} \mathrm{ClN}_{3} \mathrm{O}_{7}[\mathrm{M}+\mathrm{H}] 640.2784$, found 640.2787 .

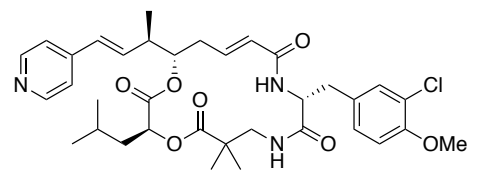

$(3 S, 10 R, 16 S, E)-10-(3-c h l o r o-4-m e t h o x y b e n z y l)-3-i s o b u t y l-6,6-$ dimethyl-16-((R,E)-4-(pyridin-4-yl)but-3-en-2-yl)-1,4-dioxa-8,11diazacyclohexadec-13-ene-2,5,9,12-tetraone (16d*). Reaction was run and purified as general procedure for semi-preparative scale reaction. Retention time: 36.5 minutes, ${ }^{1} \mathbf{H}$ NMR $\left(600 \mathrm{MHz}, \mathrm{CD}_{3} \mathrm{OD}\right) \delta 8.45$ (d, J = 5.7 Hz, 2H, 2x NCHCH unit A), 7.44 (d, J $=6.3 \mathrm{~Hz}, 2 \mathrm{H}, 2 \mathrm{x} \mathrm{CHCHC}$ unit A Ar), $7.28(\mathrm{~d}, \mathrm{~J}=2.2 \mathrm{~Hz}, 1 \mathrm{H}, \mathrm{CCHCCl}$ unit B), 7.17 (dd, J = 8.4, 2.2 Hz, $1 \mathrm{H}, \mathrm{CCHCH}$ unit B), $6.98(\mathrm{~d}, \mathrm{~J}=8.5 \mathrm{~Hz}, 1 \mathrm{H}, \mathrm{CCHCHC}$ unit B), $6.72(\mathrm{ddd}, \mathrm{J}=15.1,11.2,3.9 \mathrm{~Hz}, 1 \mathrm{H}$, $\mathrm{CH}_{2} \mathrm{CH}=\mathrm{CH}$ unit A), $6.50-6.47(\mathrm{~m}, 2 \mathrm{H}, \mathrm{CC} \boldsymbol{H}=\mathrm{C} \boldsymbol{H C H}$ unit A), $5.92(\mathrm{dd}, \mathrm{J}=15.1,1.9 \mathrm{~Hz}, 1 \mathrm{H}, \mathrm{CH}=\mathrm{C} \boldsymbol{H C O}$ unit A), 5.09 (ddd, $\mathrm{J}=11.3,7.2,1.9 \mathrm{~Hz}, 1 \mathrm{H}, \mathrm{CHCHCH}_{2}$ unit A), 4.95 (dd, J = 9.9, $3.3 \mathrm{~Hz}, 1 \mathrm{H}, \mathrm{COCHO}$ unit D), 4.51 (dd, J = 11.3, 3.7 Hz, 1H, COCHNH Unit B), 3.84 (s, 3H, COCH $\boldsymbol{H}_{3}$ unit B), 3.46 (d, J = 13.6 $\mathrm{Hz}, 1 \mathrm{H}, 1 \times \mathrm{CCH}_{2} \mathrm{NH}$ unit C), $3.18\left(\mathrm{dd}, \mathrm{J}=14.6,3.8 \mathrm{~Hz}, 1 \mathrm{H}, 1 \times \mathrm{CHCH}_{2} \mathrm{C}\right.$ unit B), $3.08(\mathrm{~d}, \mathrm{~J}=13.6 \mathrm{~Hz}, 1 \mathrm{H}$, $1 \times \mathrm{CCH}_{2} \mathrm{NH}$ unit C), 2.74 (dd, $\mathrm{J}=14.5,11.3 \mathrm{~Hz}, 1 \mathrm{H}, 1 \times \mathrm{CHCH}_{2} \mathrm{C}$ unit B), $2.74-2.66$ (m, 2H, CHCHCHO unit A \& 1x $\mathrm{CHOCCH}_{2} \mathrm{CH}$ unit A), 2.35 (dt, J = 14.5, $11.2 \mathrm{~Hz}, 1 \mathrm{H}, 1 \times$ CHOCCH $2 \mathrm{CH}$ unit A), $1.67-1.54$ $\left(\mathrm{m}, 2 \mathrm{H},\left(\mathrm{CH}_{3}\right)_{2} \mathrm{CHCH}_{2}\right.$ unit D \& 1x $\mathrm{CHCH}_{2} \mathrm{CH}$ unit D), $1.37-1.24\left(\mathrm{~m}, 1 \mathrm{H}, 1 \mathrm{x} \mathrm{CHCH} \boldsymbol{H}_{2} \mathrm{CH}\right.$ unit D), 1.19 $\left(\mathrm{s}, 3 \mathrm{H}, 1 \mathrm{x}\left(\mathrm{CH}_{3}\right)_{2} \mathrm{C}\right.$ unit $\left.\mathrm{C}\right), 1.18\left(\mathrm{~d}, \mathrm{~J}=6.9 \mathrm{~Hz}, 3 \mathrm{H}, \mathrm{CH}_{3} \mathrm{CH}\right.$ unit $\left.\mathrm{A}\right), 1.16\left(\mathrm{~s}, 3 \mathrm{H}, 1 \mathrm{x}\left(\mathrm{CH}_{3}\right)_{2} \mathrm{C}\right.$ unit $\left.\mathrm{C}\right), 0.74$ $\left(\mathrm{d}, \mathrm{J}=6.3 \mathrm{~Hz}, 3 \mathrm{H}, 1 \times\left(\mathrm{CH}_{3}\right)_{2} \mathrm{CH}\right.$ unit D), $0.71\left(\mathrm{~d}, \mathrm{~J}=6.4 \mathrm{~Hz}, 3 \mathrm{H}, 1 \mathrm{x}\left(\mathrm{CH}_{3}\right)_{2} \mathrm{CH}\right.$ unit D). ${ }^{13} \mathbf{C}$ NMR $(150$ $\left.\mathrm{MHz}, \mathrm{CD}_{3} \mathrm{OD}\right) \delta 177.6(\mathrm{OCOCH}$ unit $\mathrm{C}$ ), 174.1 (NHCOCH unit B), 172.3 (OCOCH unit D), 168.4 (CHCONH unit A), 155.3 (CHCHCC unit B), 150.4 (NCHCH unit A Ar), 147.1 (CHCCH unit A Ar), 143.6 
$\left(\mathrm{CH}_{2} \mathrm{CH}=\mathrm{CH}\right.$ unit $\left.\mathrm{A}\right), 138.6(\mathrm{CH}=\boldsymbol{C H C H}$ unit $\mathrm{A}), 132.2\left(\mathrm{CH}_{2} \boldsymbol{C C H}_{2}\right.$ unit B), $131.5(\mathrm{CCHC}$ unit B), 130.3 $(\mathrm{C} C \mathrm{H}=\mathrm{CH}$ unit $\mathrm{A}), 129.3(\mathrm{CCHCH}$ unit $\mathrm{B}), 125.5(\mathrm{CHCHCO}$ unit $\mathrm{A}), 123.2(\mathrm{CHCCl}$ Unit $\mathrm{B}), 122.5$ (CHCHC unit $\mathrm{A} \mathrm{Ar}), 113.5$ (CCHCHC unit $\mathrm{B}), 78.3\left(\mathrm{CHCHCH}_{2}\right.$ unit $\left.\mathrm{A}\right), 72.6(\mathrm{COCHO}$ unit D), 57.5 $(\mathrm{COCHNH}$ unit $\mathrm{B}), 56.6\left(\mathrm{CHOCH}_{3}\right.$ unit $\left.\mathrm{B}\right), 47.4\left(\mathrm{CCH}_{2}\right.$ unit $\left.\mathrm{C}\right), 44.0\left(\mathrm{COCCH}_{2}\right.$ unit $\left.\mathrm{C}\right), 43.7$ $\left(\mathrm{CHCHCHCH}_{2}\right.$ unit A), $40.9\left(\mathrm{CHCH}_{2} \mathrm{CH}\right.$ unit D), $37.8\left(\mathrm{CHCH}_{2} \mathrm{CH}\right.$ unit A), $36.4\left(\mathrm{CHCH}_{2} \mathrm{C}\right.$ unit B), 25.6 $\left(\left(\mathrm{CH}_{3}\right)_{2} \boldsymbol{C H C H}_{2}\right.$ unit D), $23.3\left(1 \mathrm{x} \mathrm{C}(\boldsymbol{C H})_{2} \text { unit C), } 23.3 \text { (1x C( } \boldsymbol{C H}_{3}\right)_{2}$ unit $\left.\mathrm{C}\right), 23.2\left(1 \mathrm{x}\left(\boldsymbol{C} \mathrm{H}_{3}\right)_{2} \mathrm{CH}\right.$ unit D), $21.6\left(1 \mathrm{x}\left(\mathrm{CH}_{3}\right)_{2} \mathrm{CH}\right.$ unit D), $17.5\left(1 \mathrm{x} \mathrm{CH}_{3} \mathrm{CH}\right.$ unit A). HRMS (ESI) calcd for $\mathrm{C}_{35} \mathrm{H}_{44} \mathrm{ClN}_{3} \mathrm{O}_{7}[\mathrm{M}+\mathrm{H}]$ 654.2941 , found 654.2947.

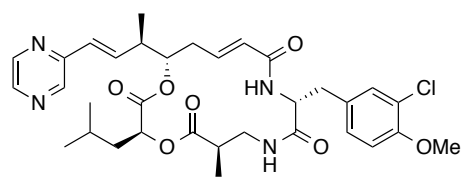

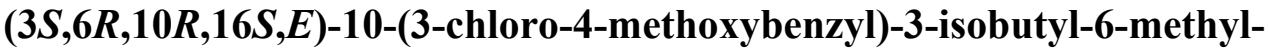
16-((R,E)-4-(pyrazin-2-yl)but-3-en-2-yl)-1,4-dioxa-8,11diazacyclohexadec-13-ene-2,5,9,12-tetraone (16e). Reaction was run and purified as general procedure for semi-preparative scale reaction. Retention time: 36 minutes, ${ }^{1} \mathbf{H}$ NMR $\left(600 \mathrm{MHz}, \mathrm{CD}_{3} \mathrm{OD}\right) \delta 8.61(\mathrm{~d}, \mathrm{~J}=1.5 \mathrm{~Hz}, 1 \mathrm{H}, \mathrm{CNCHCH}$ unit A Ar), 8.54 (dd, $\mathrm{J}=2.6,1.5 \mathrm{~Hz}, 1 \mathrm{H}, \mathrm{CNCHCHN}$ unit A Ar), $8.42(\mathrm{~d}, \mathrm{~J}=2.6 \mathrm{~Hz}, 1 \mathrm{H}, \mathrm{NCHC}$ unit A Ar), $7.28(\mathrm{~d}, \mathrm{~J}=2.2 \mathrm{~Hz}$, 1H, CCHCCl unit B), 7.17 (dd, J = 8.4, 2.2 Hz, 1H, CCHCH unit B), 6.98 (d, J = 8.5 Hz, 1H, CCHCHC unit B), $6.84(\mathrm{dd}, \mathrm{J}=15.7,8.9 \mathrm{~Hz}, 1 \mathrm{H}, \mathrm{CCH}=\mathrm{CHCH}$ unit A), 6.71 (ddd, $\mathrm{J}=15.2,11.2,3.9 \mathrm{~Hz}, 1 \mathrm{H}$, $\mathrm{CH}_{2} \mathrm{CH}=\mathrm{CH}$ unit A), $6.64(\mathrm{~d}, \mathrm{~J}=15.7 \mathrm{~Hz}, 1 \mathrm{H}, \mathrm{CC} \boldsymbol{H}=\mathrm{CH}$ unit $\mathrm{A}), 5.93(\mathrm{dd}, \mathrm{J}=15.1,1.9 \mathrm{~Hz}, 1 \mathrm{H}, \mathrm{CH}=\mathrm{CHCO}$ unit A), 5.11 (ddd, $\mathrm{J}=11.3,6.8,2.0 \mathrm{~Hz}, 1 \mathrm{H}, \mathrm{CHCHCH}_{2}$ unit A), 4.94 (dd, J = 9.7, 3.7 Hz, 1H, COCHO unit D), 4.53 (dd, $\mathrm{J}=11.2,3.9 \mathrm{~Hz}, 1 \mathrm{H}, \mathrm{COC} \boldsymbol{H} \mathrm{NH}$ Unit B), 3.84 (s, 3H, COCH $\boldsymbol{H}_{3}$ unit B), 3.58 (dd, J = 13.8, $3.4 \mathrm{~Hz}, 1 \mathrm{H}, 1 \times \mathrm{CHCH}_{2} \mathrm{NH}$ unit C), 3.27 (dd, J = 13.7, $3.0 \mathrm{~Hz}, 1 \mathrm{H}, 1 \times \mathrm{CHCH}_{2} \mathrm{NH}$ unit C), 3.18 (dd, J = $14.5,3.9 \mathrm{~Hz}, 1 \mathrm{H}, 1 \mathrm{x} \mathrm{CHCH}_{2} \mathrm{C}$ unit B), $2.81-2.72\left(\mathrm{~m}, 3 \mathrm{H}, \mathrm{COCHCH}_{2}\right.$ unit $\mathrm{C}, 1 \mathrm{x} \mathrm{CHCH}_{2} \mathrm{C}$ unit $\mathrm{B}$, CHCHCHO unit A), 2.69 (ddt, $\mathrm{J}=14.2,3.7,1.9 \mathrm{~Hz}, 1 \mathrm{H}, 1 \mathrm{x} \mathrm{CHOCCH}_{2} \mathrm{CH}$ unit A), 2.38 (dt, $\mathrm{J}=14.6,11.2$ $\mathrm{Hz}, 1 \mathrm{H}, 1 \times \mathrm{CHOCCH}_{2} \mathrm{CH}$ unit A), $1.70-1.57\left(\mathrm{~m}, 2 \mathrm{H},\left(\mathrm{CH}_{3}\right)_{2} \mathrm{CHCH}_{2}\right.$ unit D \& 1x $\mathrm{CHCH}_{2} \mathrm{CH}$ unit D), 1.36 (ddd, $\mathrm{J}=14.1,8.7,3.7 \mathrm{~Hz}, 1 \mathrm{H}, 1 \times \mathrm{CHCH}_{2} \mathrm{CH}$ unit D), $1.19\left(\mathrm{~d}, \mathrm{~J}=6.9 \mathrm{~Hz}, 3 \mathrm{H}, \mathrm{CH}_{3} \mathrm{CH}\right.$ unit A), 1.19 $\left(\mathrm{d}, \mathrm{J}=7.5 \mathrm{~Hz}, 3 \mathrm{H}, \mathrm{CH}_{3} \mathrm{CH}\right.$ unit C), $0.76\left(\mathrm{~d}, \mathrm{~J}=6.5 \mathrm{~Hz}, 3 \mathrm{H}, 1 \mathrm{x}\left(\mathrm{CH}_{3}\right)_{2} \mathrm{CH}\right.$ unit D), $0.73(\mathrm{~d}, \mathrm{~J}=6.5 \mathrm{~Hz}, 3 \mathrm{H}$, $1 \mathrm{x}\left(\mathrm{CH}_{3}\right)_{2} \mathrm{CH}$ unit D). ${ }^{13} \mathrm{C}$ NMR $\left(150 \mathrm{MHz}, \mathrm{CD}_{3} \mathrm{OD}\right) \delta 177.5(\mathrm{OCOCH}$ unit $\mathrm{C}), 174.0$ (NHCOCH unit B), 172.2 (OCOCH unit D), 168.3 (CHCONH unit A), 155.4 (CHCHCC unit B), 152.5 (NCCH unit A Ar), 145.7 (CNCHCHN unit A Ar), 144.2 (CNCHCH unit A Ar), $143.9\left(\mathrm{NCHC}\right.$ unit A Ar), $143.3\left(\mathrm{CH}_{2} \boldsymbol{C H}=\mathrm{CH}\right.$ unit A), $140.2\left(\mathrm{CH}=\boldsymbol{C H C H}\right.$ unit A), $132.2\left(\mathrm{CH}_{2} \mathbf{C C H}_{2}\right.$ unit B), $131.5(\mathrm{CCHC}$ unit B), $129.3(\mathrm{CCHCH}$ unit B), $128.8(\mathrm{CCH}=\mathrm{CH}$ unit $\mathrm{A}), 125.6$ ( $\mathrm{CHCHCO}$ unit $\mathrm{A}), 123.3$ (CHCCl Unit B), 113.5 (CCHCHC unit B), $78.5\left(\mathrm{CHCHCH}_{2}\right.$ unit A), 72.8 (COCHO unit D), 57.4 (COCHNH unit B), $56.6\left(\mathrm{CHOCH}_{3}\right.$ unit B), 43.4 $\left(\mathrm{CHCHCHCH}_{2}\right.$ unit A), $41.2\left(\mathrm{CHCH}_{2} \mathrm{NH}\right.$ unit C), $40.9\left(\mathrm{CHCH}_{2} \mathrm{CH}\right.$ unit D), $39.0\left(\mathrm{COCHCH}_{2}\right.$ unit C), 38.0 $\left(\mathrm{CHCH}_{2} \mathrm{CH}\right.$ unit A), $36.3\left(\mathrm{CHCH}_{2} \mathrm{C}\right.$ unit B), $25.6\left(\left(\mathrm{CH}_{3}\right)_{2} \mathrm{CHCH}_{2}\right.$ unit D), $23.2\left(1 \mathrm{x}\left(\mathrm{CH}_{3}\right)_{2} \mathrm{CH}\right.$ unit D), 21.6 (1x $\left(\boldsymbol{C H}_{3}\right)_{2} \mathrm{CH}$ unit D), 17.4 (1x $\boldsymbol{C H}_{3} \mathrm{CH}$ unit A), 15.1 (1x $\mathrm{CH}_{\boldsymbol{C}} \mathrm{H}_{3}$ unit C). HRMS (ESI) calcd for [M+H] 641.2737, found 641.2742 .

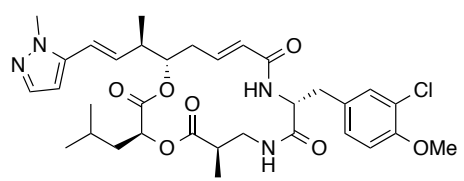

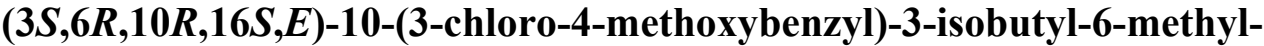
16-((R,E)-4-(1-methyl-1H-pyrazol-5-yl)but-3-en-2-yl)-1,4-dioxa-8,11diazacyclohexadec-13-ene-2,5,9,12-tetraone (16f). Reaction was run and purified as general procedure for semi-preparative scale reaction. Retention time: 34 minutes, ${ }^{1} \mathbf{H}$ NMR $\left(600 \mathrm{MHz}, \mathrm{CD}_{3} \mathrm{OD}\right) \delta 7.36(\mathrm{~d}, \mathrm{~J}=2.1 \mathrm{~Hz}, 1 \mathrm{H}, \mathrm{NCHCHC}$ unit A Ar), 7.28 (d, J $=2.2 \mathrm{~Hz}, 1 \mathrm{H}, \mathrm{CCHCCl}$ unit B), $7.17(\mathrm{dd}, \mathrm{J}=8.5,2.2 \mathrm{~Hz}, 1 \mathrm{H}, \mathrm{CCHCH}$ unit B), $6.98(\mathrm{~d}, \mathrm{~J}=8.4 \mathrm{~Hz}, 1 \mathrm{H}$, CCHCHC unit B), 6.71 (ddd, $\mathrm{J}=15.1,11.2,3.9 \mathrm{~Hz}, 1 \mathrm{H}, \mathrm{CH}_{2} \mathrm{CH}=\mathrm{CH}$ unit A), $6.52(\mathrm{~d}, \mathrm{~J}=15.8 \mathrm{~Hz}, 1 \mathrm{H}$, $\mathrm{NCHCHC}$ unit A Ar), $6.42(\mathrm{~d}, \mathrm{~J}=2.1 \mathrm{~Hz}, 1 \mathrm{H}, \mathrm{CCH}=\mathrm{CH}$ unit A), $6.14(\mathrm{dd}, \mathrm{J}=15.8,9.0 \mathrm{~Hz}, 1 \mathrm{H}$, $\mathrm{CCH}=\mathrm{C} \boldsymbol{H C H}$ unit A), $5.93(\mathrm{dd}, \mathrm{J}=15.1,1.9 \mathrm{~Hz}, 1 \mathrm{H}, \mathrm{CH}=\mathrm{C} \boldsymbol{H C O}$ unit A), 5.07 (ddd, $\mathrm{J}=11.3,6.9,1.9 \mathrm{~Hz}$, $1 \mathrm{H}, \mathrm{CHCHCH}_{2}$ unit A), 4.93 (dd, J = 9.8, 3.6 Hz, 1H, COCHO unit D), 4.52 (dd, J = 11.3, 3.9 Hz, 1H, $\mathrm{COCHNH}$ Unit B), 3.84 (s, 6H, $\mathrm{COCH}_{3}$ unit B), 3.59 (dd, J = 13.8, $3.3 \mathrm{~Hz}, 1 \mathrm{H}, 1 \times \mathrm{CHCH}_{2} \mathrm{NH}$ unit C), $3.27\left(\mathrm{dd}, \mathrm{J}=13.8,3.0 \mathrm{~Hz}, 1 \mathrm{H}, 1 \times \mathrm{CHCH}_{2} \mathrm{NH}\right.$ unit C), $3.18\left(\mathrm{dd}, \mathrm{J}=14.5,3.9 \mathrm{~Hz}, 1 \mathrm{H}, 1 \mathrm{x} \mathrm{CHCH}_{2} \mathrm{C}\right.$ unit B), 
$2.79-2.62\left(\mathrm{~m}, 4 \mathrm{H}, \mathrm{COCHCH}_{2}\right.$ unit $\mathrm{C}, 1 \times \mathrm{CHCH}_{2} \mathrm{C}$ unit $\mathrm{B}, \mathrm{CHCHCHO}$ unit $\mathrm{A}, \&$ 1x $\mathrm{CHOCCH}_{2} \mathrm{CH}$ unit A), $2.35\left(\mathrm{dt}, \mathrm{J}=14.6,11.2 \mathrm{~Hz}, 1 \mathrm{H}, 1 \mathrm{x} \mathrm{CHOCCH}_{2} \mathrm{CH}\right.$ unit A), $1.71-1.57\left(\mathrm{~m}, 2 \mathrm{H},\left(\mathrm{CH}_{3}\right)_{2} \mathrm{CHCH}_{2}\right.$ unit D \& $1 \mathrm{x} \mathrm{CHCH}_{2} \mathrm{CH}$ unit D), 1.35 (ddd, $\mathrm{J}=14.0,8.6,3.5 \mathrm{~Hz}, 1 \mathrm{H}, 1 \mathrm{x} \mathrm{CHCH}_{2} \mathrm{CH}$ unit D), 1.19 (d, J = 7.4 Hz, 3H, $\mathrm{CH}_{3} \mathrm{CH}$ unit A), $1.17\left(\mathrm{~d}, \mathrm{~J}=6.9 \mathrm{~Hz}, 3 \mathrm{H}, \mathrm{CH}_{3} \mathrm{CH}\right.$ unit $\left.\mathrm{C}\right), 0.77\left(\mathrm{~d}, \mathrm{~J}=6.4 \mathrm{~Hz}, 6 \mathrm{H},(\mathrm{CH})_{2} \mathrm{CH}\right.$ unit D). ${ }^{13} \mathrm{C}$ NMR: $\left(150 \mathrm{MHz}, \mathrm{CD}_{3} \mathrm{OD}\right) \delta 177.6(\mathrm{OCOCH}$ unit $\mathrm{C}), 174.0(\mathrm{NHCOCH}$ unit $\mathrm{B}), 172.2(\mathrm{OCOCH}$ unit D), 168.3 (CHCONH unit A), $155.4\left(\mathrm{CHCHCC}\right.$ unit B), $143.4\left(\mathrm{CH}_{2} \mathrm{CH}=\mathrm{CH}\right.$ unit A), $142.29(\mathrm{NCCH}$ unit $\mathrm{A}$ $\mathrm{Ar}), 139.3(\mathrm{NCHCH}$ unit $\mathrm{A} \mathrm{Ar}), 136.8(\mathrm{CCH}=\boldsymbol{C H C H}$ unit $\mathrm{A}), 132.2\left(\mathrm{CH}_{2} \boldsymbol{C C H}_{2}\right.$ unit B), $131.5(\mathrm{CCHC}$ unit B), 129.3 (CCHCH unit B), 125.6 (CHCHCO unit A), 123.2 ( $\mathrm{CHCCl}$ Unit B), $119.2(\mathrm{CCH}=\mathrm{CH}$ unit A), 113.5 (CCHCHC unit B), 103.7 ( $\mathrm{CHCHC}$ unit $\mathrm{A} \mathrm{Ar}), 78.4\left(\mathrm{CHCHCH}_{2}\right.$ unit $\left.\mathrm{A}\right), 72.8(\mathrm{COCHO}$ unit D), $57.4\left(\mathrm{COCHNH}\right.$ unit B), $56.6\left(\mathrm{CHOCH}_{3}\right.$ unit B), $43.7\left(\mathrm{CHCHCHCH}_{2}\right.$ unit $\left.\mathrm{A}\right), 41.2\left(\mathrm{CHCH}_{2} \mathrm{NH}\right.$ unit $\left.\mathrm{C}\right)$, $41.0\left(\mathrm{CHCH}_{2} \mathrm{CH}\right.$ unit D), $39.0\left(\mathrm{COCHCH}_{2}\right.$ unit $\left.\mathrm{C}\right), 38.0\left(\mathrm{CHCH}_{2} \mathrm{CH}\right.$ unit $\left.\mathrm{A}\right), 36.5\left(\mathrm{CHCH}_{2} \mathrm{C}\right.$ unit B), 36.4 $\left(\boldsymbol{C} \mathrm{H}_{3} \mathrm{~N}\right.$ unit A Ar), $25.7\left(\left(\mathrm{CH}_{3}\right)_{2} \boldsymbol{C H C H}_{2}\right.$ unit D), $23.2\left(1 \mathrm{x}\left(\boldsymbol{C} \mathrm{H}_{3}\right)_{2} \mathrm{CH}\right.$ unit D), $21.6\left(1 \mathrm{x}\left(\boldsymbol{C} \mathrm{H}_{3}\right)_{2} \mathrm{CH}\right.$ unit D), 17.5 (1x $\boldsymbol{C H}_{3} \mathrm{CH}$ unit A), 15.1 ( $1 \mathrm{x} \mathrm{CH}_{C} \mathrm{H}_{3}$ unit C). HRMS (ESI) calcd for $\mathrm{C}_{33} \mathrm{H}_{43} \mathrm{ClN}_{4} \mathrm{O}_{7}[\mathrm{M}+\mathrm{H}]$ 643.2893, found 643.2890 .

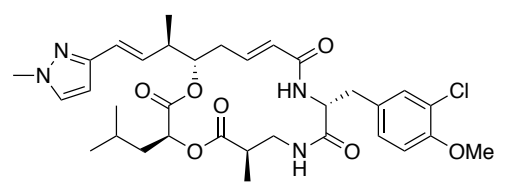

$(3 S, 6 R, 10 R, 16 S, E)-10-(3-c h l o r o-4-m e t h o x y b e n z y l)-3-i s o b u t y l-6-$ methyl-16-((R,E)-4-(1-methyl-1H-pyrazol-3-yl)but-3-en-2-yl)-1,4dioxa-8,11-diazacyclohexadec-13-ene-2,5,9,12-tetraone (16g). Reaction was run and purified as general procedure for semi-preparative scale reaction. Retention time: 36 minutes, ${ }^{1} \mathbf{H}$ NMR $\left(600 \mathrm{MHz}, \mathrm{CD}_{3} \mathrm{OD}\right) \delta 7.47$ $(\mathrm{d}, \mathrm{J}=2.3 \mathrm{~Hz}, 1 \mathrm{H}, \mathrm{NCHCHC}$ unit A Ar), 7.26 (d, J = 2.2 Hz, 1H, CCHCCl unit B), 7.15 (dd, J = 8.4, 2.2 $\mathrm{Hz}, 1 \mathrm{H}, \mathrm{CCHCH}$ unit B), 6.96 (d, J = 8.5 Hz, 1H, CCHCHC unit B), 6.68 (ddd, J = 15.1, 11.1, 3.9 Hz, 1H, $\mathrm{CH}_{2} \mathrm{CH}=\mathrm{CH}$ unit A), $6.36(\mathrm{~d}, \mathrm{~J}=16.0 \mathrm{~Hz}, 1 \mathrm{H}, \mathrm{NCHCHC}$ unit A Ar), $6.33(\mathrm{~d}, \mathrm{~J}=2.3 \mathrm{~Hz}, 1 \mathrm{H}, \mathrm{CCH}=\mathrm{CH}$ unit A), $6.05(\mathrm{dd}, \mathrm{J}=16.0,9.0 \mathrm{~Hz}, 1 \mathrm{H}, \mathrm{CH}=\mathrm{CHCH}$ unit A), $5.90(\mathrm{dd}, \mathrm{J}=15.2,1.9 \mathrm{~Hz}, 1 \mathrm{H}, \mathrm{CH}=\mathrm{CHCO}$ unit A), $5.00\left(\mathrm{ddd}, \mathrm{J}=11.3,7.1,2.0 \mathrm{~Hz}, 1 \mathrm{H}, \mathrm{CHCHCH}_{2}\right.$ unit A), 4.90 (dd, J = 10.0, 3.6 Hz, 1H, COCHO unit D), $4.50\left(\mathrm{dd}, \mathrm{J}=11.2,3.9 \mathrm{~Hz}, 1 \mathrm{H}, \mathrm{COC} \boldsymbol{H} \mathrm{NH}\right.$ Unit B), $3.82\left(\mathrm{~s}, 3 \mathrm{H}, \mathrm{COCH} \boldsymbol{H}_{3}\right.$ unit B), $3.82\left(\mathrm{~s}, 3 \mathrm{H}, \mathrm{CH}_{3} \mathrm{~N}\right.$ unit A) $3.56\left(\mathrm{dd}, \mathrm{J}=13.8,3.3 \mathrm{~Hz}, 1 \mathrm{H}, 1 \times \mathrm{CHCH}_{2} \mathrm{NH}\right.$ unit C), $3.25\left(\mathrm{dd}, \mathrm{J}=13.8,3.0 \mathrm{~Hz}, 1 \mathrm{H}, 1 \times \mathrm{CHCH}_{2} \mathrm{NH}\right.$ unit C), $3.16\left(\mathrm{dd}, \mathrm{J}=14.5,3.9 \mathrm{~Hz}, 1 \mathrm{H}, 1 \mathrm{x} \mathrm{CHCH}_{2} \mathrm{C}\right.$ unit B), $2.77-2.71$ (m, 1H, $\mathrm{COCHCH}_{2}$ unit C), 2.74 (dd, $\mathrm{J}=14.7,11.2,1 \mathrm{H}, 1 \times \mathrm{CHCH}_{2} \mathrm{C}$ unit B) $2.65\left(\mathrm{ddt}, \mathrm{J}=14.5,4.1,2.1 \mathrm{~Hz}, 1 \mathrm{H}, 1 \mathrm{x} \mathrm{CHOCCH}_{2} \mathrm{CH}\right.$ unit A), $2.61-2.51\left(\mathrm{~m}, 1 \mathrm{H}, \mathrm{CHCHCHO}\right.$ unit A), $2.34\left(\mathrm{dt}, \mathrm{J}=14.6,11.2 \mathrm{~Hz}, 1 \mathrm{H}, 1 \mathrm{x}\right.$ CHOCCH $\boldsymbol{H}_{2} \mathrm{CH}$ unit A), $1.68-1.61\left(\mathrm{~m}, 1 \mathrm{H},\left(\mathrm{CH}_{3}\right)_{2} \mathrm{CHCH}_{2}\right.$ unit D), 1.57 (ddd, $\mathrm{J}=14.8,10.0,5.0 \mathrm{~Hz}, 1 \mathrm{H}, 1 \mathrm{x} \mathrm{CHCH}_{2} \mathrm{CH}$ unit D), $1.35\left(\mathrm{ddd}, \mathrm{J}=14.2,9.0,3.6 \mathrm{~Hz}, 1 \mathrm{H}, 1 \times \mathrm{CHCH}_{2} \mathrm{CH}\right.$ unit D), 1.17 (d, J = 7.4 Hz, 3H, $\mathrm{CH}_{3} \mathrm{CH}$ unit C), 1.12 $\left(\mathrm{d}, \mathrm{J}=6.8 \mathrm{~Hz}, 3 \mathrm{H}, \mathrm{CH}_{3} \mathrm{CH}\right.$ unit A), $0.76\left(\mathrm{~d}, \mathrm{~J}=6.5 \mathrm{~Hz}, 3 \mathrm{H}, 1 \mathrm{x}\left(\mathrm{CH}_{3}\right)_{2} \mathrm{CH}\right.$ unit D), $0.74(\mathrm{~d}, \mathrm{~J}=6.6 \mathrm{~Hz}, 3 \mathrm{H}$, $1 \mathrm{x}\left(\mathrm{CH}_{3}\right)_{2} \mathrm{CH}$ unit D). ${ }^{13} \mathbf{C}$ NMR $\left(150 \mathrm{MHz}, \mathrm{CD}_{3} \mathrm{OD}\right) \delta 177.6(\mathrm{OCOCH}$ unit $\mathrm{C}), 174.1$ ( $\mathrm{NHCOCH}$ unit B), 172.3 (OCOCH unit D), 168.3 (CHCONH unit A), 155.3 (CHCHCC unit B), 151.5 ( $\mathrm{NCCH}$ unit A Ar), $143.5\left(\mathrm{CH}_{2} \boldsymbol{C H}=\mathrm{CH}\right.$ unit A), $133.6\left(\mathrm{CCH}=\boldsymbol{C H C H}\right.$ unit A), $133.2(\mathrm{NCHCHC}$ unit A Ar $), 132.2\left(\mathrm{CH}_{2} \boldsymbol{C C H}_{2}\right.$ unit B), 131.5 (CCHC unit $\mathrm{B}), 129.3(\mathrm{CCHCH}$ unit $\mathrm{B}), 125.6(\mathrm{CHCHCO}$ unit $\mathrm{A}), 124.5(\mathrm{CCH}=\mathrm{CH}$ unit $\mathrm{A})$, 123.3 (CHCCl Unit B), 113.5 (CCHCHC unit B), 103.6 (CHCHC unit A Ar), $78.5\left(\mathrm{CHCHCH}_{2}\right.$ unit A), 72.9 (COCHO unit D), $57.4\left(\mathrm{COCHNH}\right.$ unit B), $56.6\left(\mathrm{CHOCH}_{3}\right.$ unit B), $43.6\left(\mathrm{CHCHCHCH}_{2}\right.$ unit A), 41.2 $\left(\mathrm{CHCH}_{2} \mathrm{NH}\right.$ unit $\left.\mathrm{C}\right), 40.8\left(\mathrm{CHCH}_{2} \mathrm{CH}\right.$ unit D), $39.0\left(\mathrm{COCHCH}_{2}\right.$ unit C), $38.6\left(\mathrm{CH}_{3} \mathrm{~N}\right.$ unit A Ar), 37.9 $\left(\mathrm{CHCH}_{2} \mathrm{CH}\right.$ unit A), $36.4\left(\mathrm{CHCH}_{2} \mathrm{C}\right.$ unit B), $25.7\left(\left(\mathrm{CH}_{3}\right)_{2} \mathrm{CHCH}_{2}\right.$ unit D), $23.2\left(1 \mathrm{x}\left(\mathrm{CH}_{3}\right)_{2} \mathrm{CH}\right.$ unit D), 21.6 $\left(1 \mathrm{x}\left(\mathrm{CH}_{3}\right)_{2} \mathrm{CH}\right.$ unit D), $17.6\left(1 \mathrm{x} \mathrm{CH}_{3} \mathrm{CH}\right.$ unit A), 15.1 (1x $\mathrm{CHCH}_{3}$ unit C). HRMS (ESI) calcd for $\mathrm{C}_{35} \mathrm{H}_{47} \mathrm{ClN}_{4} \mathrm{O}_{7}[\mathrm{M}+\mathrm{H}]$ 671.3206, found 671.3025.

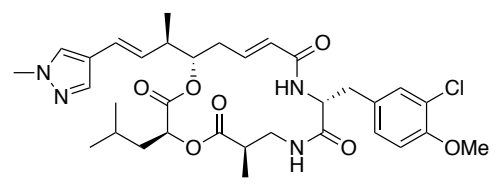

$(3 S, 6 R, 10 R, 16 S, E)-10-(3-c h l o r o-4-m e t h o x y b e n z y l)-3-i s o b u t y l-6-$ methyl-16-((R,E)-4-(1-methyl-1H-pyrazol-4-yl)but-3-en-2-yl)-1,4dioxa-8,11-diazacyclohexadec-13-ene-2,5,9,12-tetraone (16h). Reaction was run and purified as general procedure for semi-preparative scale reaction. Retention time: 36 minutest, ${ }^{1} \mathbf{H}$ NMR $\left(600 \mathrm{MHz}, \mathrm{CD}_{3} \mathrm{OD}\right) \delta 7.61$ (s, 1H, NCHC unit A Ar), 7.51 
(s, 1H, N(CH3)CHC unit A Ar), $7.28(\mathrm{~d}, \mathrm{~J}=2.2 \mathrm{~Hz}, 1 \mathrm{H}, \mathrm{CCHCCl}$ unit B), 7.17 (dd, J = 8.4, 2.2 Hz, 1H, $\mathrm{CCHCH}$ unit B), 6.98 (d, J = 8.4 Hz, 1H, CCHCHC unit B), 6.70 (ddd, $\mathrm{J}=15.2,11.2,3.9 \mathrm{~Hz}, 1 \mathrm{H}$, $\mathrm{CH}_{2} \mathrm{CH}=\mathrm{CH}$ unit A), $6.27(\mathrm{~d}, \mathrm{~J}=15.9 \mathrm{~Hz}, 1 \mathrm{H}, \mathrm{CC} \boldsymbol{H}=\mathrm{CH}$ unit $\mathrm{A}), 5.92(\mathrm{dd}, \mathrm{J}=15.2,1.9 \mathrm{~Hz}, 1 \mathrm{H}, \mathrm{CH}=\mathrm{C} \boldsymbol{H C O}$ unit A), $5.80(\mathrm{dd}, \mathrm{J}=15.9,8.9 \mathrm{~Hz}, 1 \mathrm{H}, \mathrm{CCH}=\mathrm{CHCH}$ unit A), 4.99 (ddd, $\mathrm{J}=11.3,7.1,1.9 \mathrm{~Hz}, 1 \mathrm{H}$, $\mathrm{CHCHCH}_{2}$ unit A), $4.92(\mathrm{dd}, \mathrm{J}=10.0,3.6 \mathrm{~Hz}, 1 \mathrm{H}, \mathrm{COCHO}$ unit D), $4.52(\mathrm{dd}, \mathrm{J}=11.3,3.8 \mathrm{~Hz}, 1 \mathrm{H}$, COCHNH Unit B), $3.84\left(\mathrm{~d}, \mathrm{~J}=1.8 \mathrm{~Hz}, 6 \mathrm{H}, \mathrm{COCH}_{3}\right.$ unit B), $3.58\left(\mathrm{dd}, \mathrm{J}=13.8,3.3 \mathrm{~Hz}, 1 \mathrm{H}, 1 \times \mathrm{CHCH}_{2} \mathrm{NH}\right.$ unit C), $3.27\left(\mathrm{dd}, \mathrm{J}=13.8,2.9 \mathrm{~Hz}, 1 \mathrm{H}, 1 \times \mathrm{CHCH}_{2} \mathrm{NH}\right.$ unit C), 3.18 (dd, J = 14.5, $3.8 \mathrm{~Hz}, 1 \mathrm{H}, 1 \times \mathrm{CHCH}_{2} \mathrm{C}$ unit B), $2.80-2.72\left(\mathrm{~m}, 1 \mathrm{H}, \mathrm{COCHCH}_{2}\right.$ unit $\left.\mathrm{C}\right), 2.74\left(\mathrm{dd}, \mathrm{J}=14.7,11.5,1 \mathrm{H}, 1 \mathrm{x} \mathrm{CHCH}_{2} \mathrm{C}\right.$ unit B), 2.67 (ddt, $\mathrm{J}=14.4,4.1,2.0,1 \mathrm{H}, 1 \times \mathrm{CHOCCH}_{2} \mathrm{CH}$ unit A), $2.50(\mathrm{p}, \mathrm{J}=7.1 \mathrm{~Hz}, 1 \mathrm{H}, \mathrm{CHCHCHO}$ unit A), 2.33 $\left(\mathrm{dt}, \mathrm{J}=14.5,11.2 \mathrm{~Hz}, 1 \mathrm{H}, 1 \mathrm{x} \mathrm{CHOCCH}_{2} \mathrm{CH}\right.$ unit A), $1.71-1.56\left(\mathrm{~m}, 2 \mathrm{H},\left(\mathrm{CH}_{3}\right)_{2} \mathrm{CHCH}_{2}\right.$ unit D \& $1 \mathrm{x}$ $\mathrm{CHCH}_{2} \mathrm{CH}$ unit D), 1.38 (ddd, $\mathrm{J}=13.2,9.0,3.6 \mathrm{~Hz}, 1 \mathrm{H}, 1 \times \mathrm{CHCH}_{2} \mathrm{CH}$ unit D), 1.18 (d, J = 7.5 Hz, 3H, $\mathrm{CH}_{3} \mathrm{CH}$ unit C), $1.11\left(\mathrm{~d}, \mathrm{~J}=6.8 \mathrm{~Hz}, 3 \mathrm{H}, \mathrm{CH}_{3} \mathrm{CH}\right.$ unit A), $0.78\left(\mathrm{~d}, \mathrm{~J}=6.5 \mathrm{~Hz}, 3 \mathrm{H}, 1 \mathrm{x}\left(\mathrm{CH}_{3}\right)_{2} \mathrm{CH}\right.$ unit D), $0.76\left(\mathrm{~d}, \mathrm{~J}=6.6 \mathrm{~Hz}, 3 \mathrm{H}, 1 \mathrm{x}\left(\mathrm{CH}_{3}\right)_{2} \mathrm{CH}\right.$ unit D). ${ }^{13} \mathbf{C} \mathbf{~ N M R}\left(150 \mathrm{MHz}, \mathrm{CD}_{3} \mathrm{OD}\right) \delta 177.6(\mathrm{OCOCH}$ unit $\mathrm{C})$, 174.1 ( $\mathrm{NHCOCH}$ unit $\mathrm{B}), 172.3(\mathrm{OCOCH}$ unit $\mathrm{D}), 168.4(\mathrm{CHCONH}$ unit $\mathrm{A}), 155.3$ (CHCHCC unit $\mathrm{B})$, 143.6 $\left(\mathrm{CH}_{2} \boldsymbol{C H}=\mathrm{CH}\right.$ unit A), $137.9\left(\mathrm{~N}\left(\mathrm{CH}_{3}\right) \boldsymbol{C H C}\right.$ unit $\left.\mathrm{A} \mathrm{Ar}\right), 132.2\left(\mathrm{CH}_{2} \boldsymbol{C C H}_{2}\right.$ unit B $), 131.5(\mathrm{C} \boldsymbol{C H C}$ unit B), $130.3(\mathrm{CH}=\boldsymbol{C H C H}$ unit $\mathrm{A}), 129.6(\mathrm{CCHN}$ unit $\mathrm{A} \mathrm{Ar}), 129.3(\mathrm{CCHCH}$ unit B), $125.5(\mathrm{CHCHCO}$ unit A), 123.2 ( $\mathrm{CHCCl}$ Unit $\mathrm{B}), 122.5(\mathrm{CCH}=\mathrm{CH}$ unit $\mathrm{A}), 122.0(\mathrm{CHCCH}$ unit $\mathrm{A}), 113.5(\mathrm{CCHCHC}$ unit $\mathrm{B})$, $78.7\left(\mathrm{CHCHCH}_{2}\right.$ unit A), 72.9 (COCHO unit D), $57.4\left(\mathrm{COCHNH}\right.$ unit B), $56.6\left(\mathrm{CHOCH}_{3}\right.$ unit B), 43.7 $\left(\mathrm{CHCHCHCH}_{2}\right.$ unit A), $41.2\left(\mathrm{CHCH}_{2} \mathrm{NH}\right.$ unit $\left.\mathrm{C}\right), 40.9\left(\mathrm{CHCH}_{2} \mathrm{CH}\right.$ unit D), $38.9\left(\mathrm{COCHCH}_{2}\right.$ unit C), 38.7 $\left(\boldsymbol{C} \mathrm{H}_{3} \mathrm{~N}\right.$ unit A Ar), $37.8\left(\mathrm{CHCH}_{2} \mathrm{CH}\right.$ unit A), $36.4\left(\mathrm{CHCH}_{2} \mathrm{C}\right.$ unit B), $25.6\left(\left(\mathrm{CH}_{3}\right)_{2} \boldsymbol{C H C H}_{2}\right.$ unit D), $23.2(1 \mathrm{x}$ $\left(\boldsymbol{C H}_{3}\right)_{2} \mathrm{CH}$ unit D), $21.6\left(1 \mathrm{x}\left(\boldsymbol{C H}_{3}\right)_{2} \mathrm{CH}\right.$ unit D), 17.7 (1x $\boldsymbol{C H} \mathrm{H}_{3} \mathrm{CH}$ unit A), 15.1 (1x $\mathrm{CHCH}_{3}$ unit C). HRMS (ESI) calcd for $\mathrm{C}_{33} \mathrm{H}_{43} \mathrm{ClN}_{4} \mathrm{O}_{7}[\mathrm{M}+\mathrm{H}]$ 643.2893, found 643.2890.

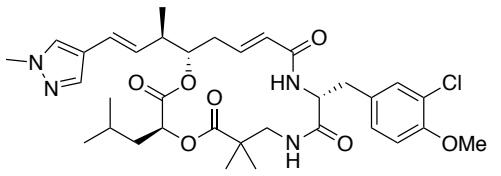

(3S,10R,16S,E)-10-(3-chloro-4-methoxybenzyl)-3-isobutyl-6,6dimethyl-16-((R,E)-4-(1-methyl-1H-pyrazol-4-yl)but-3-en-2-yl)-1,4dioxa-8,11-diazacyclohexadec-13-ene-2,5,9,12-tetraone $\quad\left(16 h^{*}\right)$

Reaction was run and purified as general procedure for semi-preparative scale reaction. Retention time: 40 minutes, ${ }^{1} \mathbf{H}$ NMR $\left(600 \mathrm{MHz}, \mathrm{CD}_{3} \mathrm{OD}\right)$ $7.60\left(\mathrm{~s}, 1 \mathrm{H}, \mathrm{CCHN}\right.$ unit A Ar), $7.51\left(\mathrm{~s}, 1 \mathrm{H}, \mathrm{N}\left(\mathrm{CH}_{3}\right) \mathrm{CHC}\right.$ unit A Ar), $7.27(\mathrm{~d}, \mathrm{~J}=2.1 \mathrm{~Hz}, 1 \mathrm{H}, \mathrm{CCHCCl}$ unit B), 7.16 (dd, J = 8.5, 2.2 Hz, 1H, CCHCH unit B), 6.97 (d, J = 8.5 Hz, 1H, CCHCHC unit B), 6.70 (ddd, J $=15.2,11.2,3.9 \mathrm{~Hz}, 1 \mathrm{H}, \mathrm{CH}_{2} \mathrm{CH}=\mathrm{CH}$ unit A), $6.27(\mathrm{~d}, \mathrm{~J}=15.9 \mathrm{~Hz}, 1 \mathrm{H}, \mathrm{CC} \boldsymbol{H}=\mathrm{CH}$ unit A $), 5.90(\mathrm{dd}, \mathrm{J}=$ 15.1, $1.9 \mathrm{~Hz}, 1 \mathrm{H}, \mathrm{CH}=\mathrm{CHCO}$ unit A), $5.79(\mathrm{dd}, \mathrm{J}=15.9,8.9 \mathrm{~Hz}, 1 \mathrm{H}, \mathrm{CH}=\mathrm{CHCH}$ unit A), 4.97 (ddd, $\mathrm{J}=$ 11.3, 7.1, $1.6 \mathrm{~Hz}, 1 \mathrm{H}, \mathrm{CHCHCH}_{2}$ unit A), 4.94 (dd, J = 9.9, $3.4 \mathrm{~Hz}, 1 \mathrm{H}, \mathrm{COCHO}$ unit D), 4.50 (dd, J = 11.4, $3.7 \mathrm{~Hz}, 1 \mathrm{H}, \mathrm{COCHNH}$ Unit B), $3.83\left(\mathrm{~s}, 3 \mathrm{H}, \mathrm{COCH}_{3}\right.$ unit B), 3.83 (s, 3H, CH $\boldsymbol{H}_{3} \mathrm{~N}$ unit A), 3.45 (d, J = $13.6 \mathrm{~Hz}, 1 \mathrm{H}, 1 \times \mathrm{CCH}_{2} \mathrm{NH}$ unit C), 3.17 (dd, J = 14.5, 3.7 Hz, 1H, 1x CHCH $\mathrm{H}_{2} \mathrm{C}$ unit B), 3.07 (d, J = 13.6 $\mathrm{Hz}, 1 \mathrm{H}, 1 \times \mathrm{CCH}_{2} \mathrm{NH}$ unit C), 2.73 (dd, $\mathrm{J}=14.5,11.4 \mathrm{~Hz}, 1 \mathrm{H}, 1 \mathrm{x} \mathrm{CHCH}_{2} \mathrm{C}$ unit B), 2.65 (dt, J = 14.7, 2.4 $\mathrm{Hz}, 1 \mathrm{H}, 1 \times \mathrm{CHOCCH}_{2} \mathrm{CH}$ unit A), 2.50 (h, J = 7.2 Hz, 1H, CHCHCHO unit A), 2.32 (dt, J = 14.4, 11.2 Hz, $1 \mathrm{H}, 1 \times \mathrm{CHOCCH}_{2} \mathrm{CH}$ unit A), $1.68-1.57\left(\mathrm{~m}, 2 \mathrm{H},\left(\mathrm{CH}_{3}\right)_{2} \mathrm{CHCH}_{2}\right.$ unit D \& 1x $\mathrm{CHCH}_{2} \mathrm{CH}$ unit D), $1.43-$ $1.36\left(\mathrm{~m}, 1 \mathrm{H}, 1 \mathrm{x} \mathrm{CHCH}_{2} \mathrm{CH}\right.$ unit D), 1.19 (s, 3H, 1 x $\left(\mathrm{CH}_{3}\right)_{2} \mathrm{C}$ unit $\left.\mathrm{C}\right), 1.15$ (s, 3H, 1 x $\left(\mathrm{CH}_{3}\right)_{2} \mathrm{C}$ unit $\left.\mathrm{C}\right), 1.11$ $\left(\mathrm{d}, \mathrm{J}=6.9 \mathrm{~Hz}, 3 \mathrm{H}, \mathrm{CH}_{3} \mathrm{CH}\right.$ unit $\left.\mathrm{A}\right), 0.77\left(\mathrm{t}, \mathrm{J}=6.7 \mathrm{~Hz}, 6 \mathrm{H},\left(\mathrm{CH}_{3}\right)_{2} \mathrm{CH}\right.$ unit D). ${ }^{13} \mathbf{C ~ N M R}(150 \mathrm{MHz}$, $\left.\mathrm{CD}_{3} \mathrm{OD}\right) \delta 179.0(\mathrm{OCOCH}$ unit $\mathrm{C}), 173.7$ ( $\mathrm{NHCOCH}$ unit $\left.\mathrm{B}\right), 172.1$ (OCOCH unit D), $168.3(\mathrm{CHCONH}$ unit A), $155.4(\mathrm{CHCHCC}$ unit $\mathrm{B}), 143.8\left(\mathrm{CH}_{2} \boldsymbol{C H}=\mathrm{CH}\right.$ unit $\left.\mathrm{A}\right), 137.9\left(\mathrm{~N}\left(\mathrm{CH}_{3}\right) \boldsymbol{C H C}\right.$ unit A Ar), 132.2 $\left(\mathrm{CH}_{2} \mathrm{CCH}_{2}\right.$ unit B), $131.5(\mathrm{CCHC}$ unit B), $130.3(\mathrm{CH}=\boldsymbol{C H C H}$ unit $\mathrm{A}), 129.6(\mathrm{CCHN}$ unit A Ar), 129.3 ( $\mathrm{CCHCH}$ unit $\mathrm{B}), 125.3$ ( $\mathrm{CHCHCO}$ unit $\mathrm{A}), 123.3$ ( $\mathrm{CHCCl}$ Unit $\mathrm{B}), 122.5(\mathrm{CCH}=\mathrm{CH}$ unit $\mathrm{A}), 122.0$ $(\mathrm{CHCCH}$ unit $\mathrm{A}), 113.5(\mathrm{CCHCHC}$ unit $\mathrm{B}), 78.7\left(\mathrm{CHCHCH}_{2}\right.$ unit $\left.\mathrm{A}\right), 72.7$ ( $\mathrm{COCHO}$ unit $\left.\mathrm{D}\right), 57.5$ (COCHNH unit B), $56.6\left(\mathrm{CHOCH}_{3}\right.$ unit B), $47.4\left(\mathrm{CHCH}_{2} \mathrm{NH}\right.$ unit $\left.\mathrm{C}\right), 44.0\left(\mathrm{COCHCH}_{2}\right.$ unit $\left.\mathrm{C}\right), 43.7$ $\left(\mathrm{CHCHCHCH}_{2}\right.$ unit A), $40.8\left(\mathrm{CHCH}_{2} \mathrm{CH}\right.$ unit D), $38.7\left(\mathrm{CH}_{3} \mathrm{~N}\right.$ unit $\left.\mathrm{A} \mathrm{Ar}\right), 37.9\left(\mathrm{CHCH}_{2} \mathrm{CH}\right.$ unit $\left.\mathrm{A}\right), 36.5$ $\left(\mathrm{CHCH}_{2} \mathrm{C}\right.$ unit B), $25.9\left(\left(\mathrm{CH}_{3}\right)_{2} \boldsymbol{C H C H}_{2}\right.$ unit D), $23.3\left(1 \mathrm{x}\left(\boldsymbol{C} \mathrm{H}_{3}\right)_{2} \mathrm{CH}\right), 23.3\left(1 \mathrm{x} \mathrm{C}\left(\boldsymbol{C H}_{3}\right)_{2}\right.$ unit $\left.\mathrm{C}\right), 23.2(1 \mathrm{x}$ 
$(\boldsymbol{C H})_{2} \mathrm{CH}$ unit D), $21.6\left(1 \mathrm{x}\left(\mathrm{CH}_{3}\right)_{2} \mathrm{CH}\right.$ unit D), 17.7 (1x $\boldsymbol{C H}_{3} \mathrm{CH}$ unit A). HRMS (ESI) calcd for $\mathrm{C}_{34} \mathrm{H}_{45} \mathrm{ClN}_{4} \mathrm{O}_{7}[\mathrm{M}+\mathrm{H}]$ 656.2977, found 656.2973.

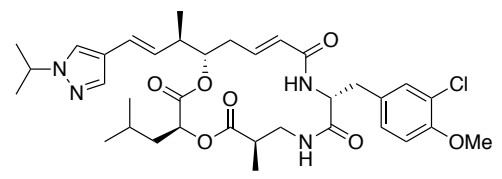

(3S,6R,10R,16S,E)-10-(3-chloro-4-methoxybenzyl)-3-isobutyl-16((R,E)-4-(1-isopropyl-1H-pyrazol-4-yl)but-3-en-2-yl)-6-methyl-1,4dioxa-8,11-diazacyclohexadec-13-ene-2,5,9,12-tetraone (16i). Reaction was run and purified as general procedure for semi-preparative scale reaction. Retention time: 42 minutes, ${ }^{1} \mathbf{H}$ NMR $\left(600 \mathrm{MHz}, \mathrm{CD}_{3} \mathrm{OD}\right) \delta 7.70$ (s, 1H, NCHC unit A Ar), 7.53 $\left(\mathrm{s}, 1 \mathrm{H}, \mathrm{N}\left(\mathrm{CH}_{3}\right) \mathrm{CHC}\right.$ unit A Ar), $7.28(\mathrm{~d}, \mathrm{~J}=2.2 \mathrm{~Hz}, 1 \mathrm{H}, \mathrm{CCHCCl}$ unit B), 7.17 (dd, J = 8.4, 2.2 Hz, 1H, $\mathrm{CCHCH}$ unit B), $6.98(\mathrm{~d}, \mathrm{~J}=8.5 \mathrm{~Hz}, 1 \mathrm{H}, \mathrm{CCHCHC}$ unit $\mathrm{B}), 6.70(\mathrm{ddd}, \mathrm{J}=15.1,11.2,3.9 \mathrm{~Hz}, 1 \mathrm{H}$, $\mathrm{CH}_{2} \mathrm{CH}=\mathrm{CH}$ unit A), $6.28(\mathrm{~d}, \mathrm{~J}=15.8 \mathrm{~Hz}, 1 \mathrm{H}, \mathrm{CC} \boldsymbol{H}=\mathrm{CH}$ unit $\mathrm{A}), 5.92(\mathrm{dd}, \mathrm{J}=15.2,1.9 \mathrm{~Hz}, 1 \mathrm{H}, \mathrm{CH}=\mathrm{C} \boldsymbol{H C O}$ unit A), $5.79\left(\mathrm{dd}, \mathrm{J}=15.9,8.9 \mathrm{~Hz}, 1 \mathrm{H}, \mathrm{CH}=\mathrm{C} \boldsymbol{H C H}\right.$ unit A), $4.98\left(\mathrm{ddd}, \mathrm{J}=11.2,7.5,2.0 \mathrm{~Hz}, 1 \mathrm{H}, \mathrm{CHCHCH}_{2}\right.$ unit A), 4.91 (dd, $\mathrm{J}=10.0,3.5 \mathrm{~Hz}, 1 \mathrm{H}, \mathrm{COCHO}$ unit D), $4.52(\mathrm{dd}, \mathrm{J}=11.2,3.8 \mathrm{~Hz}, 1 \mathrm{H}, \mathrm{COCHNH}$ Unit B), $4.47\left(\mathrm{p}, \mathrm{J}=6.7 \mathrm{~Hz}, 1 \mathrm{H},\left(\mathrm{CH}_{3}\right)_{2} \mathrm{CHN}\right), 3.84\left(\mathrm{~s}, 3 \mathrm{H}, \mathrm{COCH}_{3}\right.$ unit B), $3.58(\mathrm{dd}, \mathrm{J}=13.8,3.3 \mathrm{~Hz}, 1 \mathrm{H}, 1 \mathrm{x}$ $\mathrm{CHCH}_{2} \mathrm{NH}$ unit C), 3.27 (dd, J = 13.7, $3.0 \mathrm{~Hz}, 1 \mathrm{H}, 1 \times \mathrm{CHCH}_{2} \mathrm{NH}$ unit C), 3.18 (dd, J = 14.5, 3.8 Hz, 1H, $1 \mathrm{x} \mathrm{CHCH}_{2} \mathrm{C}$ unit B), $2.79-2.71\left(\mathrm{~m}, 1 \mathrm{H}, \mathrm{COCHCH}_{2}\right.$ unit C), $2.74\left(\mathrm{dd}, \mathrm{J}=14.2,11.0,1 \mathrm{H}, 1 \mathrm{x} \mathrm{CHCH}_{2} \mathrm{C}\right.$ unit B), 2.67 (ddt, $\mathrm{J}=14.4,4.1,2.0,1 \mathrm{H}, 1 \mathrm{x} \mathrm{CHOCCH}_{2} \mathrm{CH}$ unit A), 2.50 (p, J = 7.2 Hz, 1H, CHCHCHO unit A), 2.33 (dt, J = 14.5, $11.2 \mathrm{~Hz}, 1 \mathrm{H}, 1 \mathrm{x} \mathrm{CHOCCH} \boldsymbol{H}_{2} \mathrm{CH}$ unit A), $1.72-1.54\left(\mathrm{~m}, 2 \mathrm{H},\left(\mathrm{CH}_{3}\right)_{2} \mathrm{CHCH}_{2}\right.$ unit $\mathrm{D} \& 1 \times \mathrm{CHCH}_{2} \mathrm{CH}$ unit D), $1.46\left(\mathrm{~d}, \mathrm{~J}=6.7 \mathrm{~Hz}, 6 \mathrm{H}, 2 \mathrm{x}\left(\mathrm{CH}_{3}\right)_{2} \mathrm{CH}\right.$ unit A), 1.37 (ddd, $\mathrm{J}=14.2,9.0,3.6 \mathrm{~Hz}$, $1 \mathrm{H}, 1 \times \mathrm{CHCH}_{2} \mathrm{CH}$ unit D), $1.18\left(\mathrm{~d}, \mathrm{~J}=7.4 \mathrm{~Hz}, 3 \mathrm{H}, \mathrm{CH}_{3} \mathrm{CH}\right.$ unit $\left.\mathrm{C}\right), 1.12\left(\mathrm{~d}, \mathrm{~J}=6.9 \mathrm{~Hz}, 3 \mathrm{H}, \mathrm{CH}_{3} \mathrm{CH}\right.$ unit A), $0.77\left(\mathrm{~d}, \mathrm{~J}=6.5 \mathrm{~Hz}, 3 \mathrm{H}, 1 \times\left(\mathrm{CH}_{3}\right)_{2} \mathrm{CH}\right.$ unit D), $0.74\left(\mathrm{~d}, \mathrm{~J}=6.6 \mathrm{~Hz}, 3 \mathrm{H}, 1 \mathrm{x}\left(\mathrm{CH}_{3}\right)_{2} \mathrm{CH}\right.$ unit D). ${ }^{13} \mathbf{C ~ N M R}$ (150 MHz, $\left.\mathrm{CD}_{3} \mathrm{OD}\right) \delta 177.6(\mathrm{OCOCH}$ unit $\mathrm{C}), 174.1$ (NHCOCH unit B), 172.3 (OCOCH unit D), 168.4

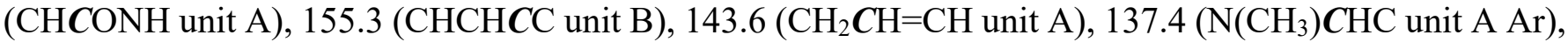
$132.2\left(\mathrm{CH}_{2} \mathrm{CCH}_{2}\right.$ unit B), $131.5(\mathrm{CCHC}$ unit B), $130.1(\mathrm{CH}=\boldsymbol{C H C H}$ unit A), $129.3(\mathrm{CCHCH}$ unit B), 126.3 ( $\mathrm{CCHCH}$ unit $\mathrm{B}), 125.5$ ( $\mathrm{CHCHCO}$ unit $\mathrm{A}), 123.3$ ( $\mathrm{CHCCl}$ Unit $\mathrm{B}), 122.7(\mathrm{CCH}=\mathrm{CH}$ unit A), 121.4 ( $\mathrm{CHCCH}$ unit A), 113.5 (CCHCHC unit $\mathrm{B}), 78.7\left(\mathrm{CHCHCH}_{2}\right.$ unit A), 72.9 ( $\mathrm{COCHO}$ unit $\left.\mathrm{D}\right), 57.4$ (COCHNH unit B), $56.6\left(\mathrm{CHOCH}_{3}\right.$ unit B), $55.1\left(\left(\mathrm{CH}_{3}\right)_{2} \mathrm{CHN}\right.$ unit $\left.\mathrm{A}\right), 43.8\left(\mathrm{CHCHCHCH}_{2}\right.$ unit A), 41.2 $\left(\mathrm{CHCH}_{2} \mathrm{NH}\right.$ unit $\left.\mathrm{C}\right), 40.9\left(\mathrm{CHCH}_{2} \mathrm{CH}\right.$ unit D), $39.0\left(\mathrm{COCHCH}_{2}\right.$ unit $\left.\mathrm{C}\right), 37.9\left(\mathrm{CHCH}_{2} \mathrm{CH}\right.$ unit A), 36.4 $\left(\mathrm{CH}_{\boldsymbol{C}} \mathrm{H}_{2} \mathrm{C}\right.$ unit B), $25.6\left(\left(\mathrm{CH}_{3}\right)_{2} \boldsymbol{C H C H}_{2}\right.$ unit D), 23.2 (1x $\left(\boldsymbol{C H}_{3}\right)_{2} \mathrm{CH}$ unit D), $21.6\left(1 \mathrm{x}\left(\boldsymbol{C H}_{3}\right)_{2} \mathrm{CH}\right.$ unit D), 17.8 (1x $\mathrm{CH}_{3} \mathrm{CH}$ unit A), 15.1 (1x $\mathrm{CHCH}_{3}$ unit C). HRMS (ESI) calcd for $\mathrm{C}_{35} \mathrm{H}_{47} \mathrm{ClN}_{4} \mathrm{O}_{7}[\mathrm{M}+\mathrm{H}]$ 671.3206, found 671.3025 .

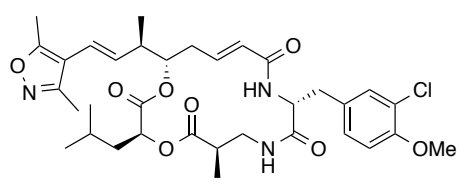

$(3 S, 6 R, 10 R, 16 S, E)-10-(3-c h l o r o-4-m e t h o x y b e n z y l)-16-((R, E)-4-(3,5-$ dimethylisoxazol-4-yl)but-3-en-2-yl)-3-isobutyl-6-methyl-1,4-dioxa-8,11diazacyclohexadec-13-ene-2,5,9,12-tetraone (16j). Reaction was run and purified as general procedure for semi-preparative scale reaction. Retention time: 42 minutes, ${ }^{1} \mathbf{H}$ NMR $\left(600 \mathrm{MHz}, \mathrm{CD}_{3} \mathrm{OD}\right) \delta 7.28(\mathrm{~d}, \mathrm{~J}=2.2 \mathrm{~Hz}, 1 \mathrm{H}, \mathrm{CCHCCl}$ unit B), 7.17 (dd, J = 8.4, $2.2 \mathrm{~Hz}, 1 \mathrm{H}, \mathrm{CCHCH}$ unit B), 6.98 (d, J = 8.4 Hz, 1H, CCHCHC unit B), 6.69 (ddd, J = 15.1, 11.1, 3.9 $\mathrm{Hz}, 1 \mathrm{H}, \mathrm{CH}_{2} \mathrm{CH}=\mathrm{CH}$ unit A), $6.23(\mathrm{~d}, \mathrm{~J}=16.2 \mathrm{~Hz}, 1 \mathrm{H}, \mathrm{CC} \boldsymbol{H}=\mathrm{CH}$ unit A), 5.93 (dd, J = 15.1, 1.9 Hz, $1 \mathrm{H}$, $\mathrm{CH}=\mathrm{CHCO}$ unit A), 5.80 (dd, $\mathrm{J}=16.2,8.9 \mathrm{~Hz}, 1 \mathrm{H}, \mathrm{CH}=\mathrm{CHCH}$ unit A), 5.06 (ddd, $\mathrm{J}=11.3,6.7,1.9 \mathrm{~Hz}$, $1 \mathrm{H}, \mathrm{CHCHCH}_{2}$ unit A), 4.93 (dd, $\mathrm{J}=9.7,3.8 \mathrm{~Hz}, 1 \mathrm{H}, \mathrm{COCHO}$ unit D), 4.51 (dd, $\mathrm{J}=11.2,3.9 \mathrm{~Hz}, 1 \mathrm{H}$, COCHNH Unit B), 3.84 (s, 3H, $\mathrm{COCH}_{3}$ unit B), 3.57 (dd, J = 13.7, 3.3 Hz, 1H, 1x CHCH $\mathrm{H}_{2} \mathrm{NH}$ unit C), $3.27\left(\mathrm{dd}, \mathrm{J}=13.8,3.0 \mathrm{~Hz}, 1 \mathrm{H}, 1 \times \mathrm{CHCH}_{2} \mathrm{NH}\right.$ unit $\left.\mathrm{C}\right), 3.17\left(\mathrm{dd}, \mathrm{J}=14.5,3.9 \mathrm{~Hz}, 1 \mathrm{H}, 1 \times \mathrm{CHCH}_{2} \mathrm{C}\right.$ unit B), $2.80-2.71\left(\mathrm{~m}, 1 \mathrm{H}, \mathrm{COCHCH}_{2}\right.$ unit C) $2.74\left(\mathrm{dd}, \mathrm{J}=14.4,11.2,1 \mathrm{H}, 1 \mathrm{x} \mathrm{CHCH} \mathrm{H}_{2} \mathrm{C}\right.$ unit B), 2.67 (ddt, $\mathrm{J}=$ 14.6, 4.0, $2.0 \mathrm{~Hz}, 1 \mathrm{H}, 1 \times \mathrm{CHOCCH}_{2} \mathrm{CH}$ unit A), 2.58 (dt, J = 8.8, 6.6 Hz, 1H, CHCHCHO unit A), 2.40 (s, $3 \mathrm{H}, \mathrm{CH}_{3} \mathrm{CC}$ unit A), 2.35 (dt, $\mathrm{J}=14.5,11.4 \mathrm{~Hz}, 1 \mathrm{H}, 1 \times \mathrm{CHOCCH}_{2} \mathrm{CH}$ unit A), 2.28 (s, 3H, $\mathrm{CH}_{3} \mathrm{CC}_{\text {unit }}$ A), $1.70-1.53\left(\mathrm{~m}, 2 \mathrm{H},\left(\mathrm{CH}_{3}\right)_{2} \mathrm{CHCH}_{2}\right.$ unit D \& 1x $\mathrm{CHCH}_{2} \mathrm{CH}$ unit D), 1.33 (ddd, $\mathrm{J}=14.1,8.7,3.8 \mathrm{~Hz}$, $1 \mathrm{H}, 1 \times \mathrm{CHCH}_{2} \mathrm{CH}$ unit D), $1.18\left(\mathrm{~d}, \mathrm{~J}=7.4 \mathrm{~Hz}, 3 \mathrm{H}, \mathrm{CH}_{3} \mathrm{CH}\right.$ unit $\left.\mathrm{C}\right), 1.15\left(\mathrm{~d}, \mathrm{~J}=6.9 \mathrm{~Hz}, 3 \mathrm{H}, \mathrm{CH} \mathrm{CH}_{3}\right.$ unit A), $0.79\left(\mathrm{~d}, \mathrm{~J}=3.7 \mathrm{~Hz}, 3 \mathrm{H}, 1 \times\left(\mathrm{CH}_{3}\right)_{2} \mathrm{CH}\right.$ unit D), $0.78\left(\mathrm{~d}, \mathrm{~J}=3.8 \mathrm{~Hz}, 3 \mathrm{H}, 1 \times\left(\mathrm{CH}_{3}\right)_{2} \mathrm{CH}\right.$ unit D). ${ }^{13} \mathbf{C} \mathbf{N M R}$ 
(150 MHz, $\left.\mathrm{CD}_{3} \mathrm{OD}\right) \delta 177.5$ (OCOCH unit $\mathrm{C}$ ), 174.0 (NHCOCH unit B), 172.2 (OCOCH unit D), 168.3 (CHCONH unit A), 166.9 (OCC unit A Ar), 159.5 (NCC unit A Ar), 155.3 (CHCHCC unit B), 143.4 $\left(\mathrm{CH}_{2} \boldsymbol{C H}=\mathrm{CH}\right.$ unit $\left.\mathrm{A}\right), 134.4(\mathrm{CH}=\boldsymbol{C H C H}$ unit $\mathrm{A}), 132.2\left(\mathrm{CH}_{2} \mathbf{C C H}_{2}\right.$ unit B), $131.5(\mathrm{CCHC}$ unit B), 129.3 ( $\mathrm{C} C H C H$ unit $\mathrm{B}), 125.6$ (CHCHCO unit A), 123.2 ( $\mathrm{CHCCl}$ Unit B), $120.2(\mathrm{CCH}=\mathrm{CH}$ unit A), 113.8 (CCHCHC unit B), 113.5 (OCCC unit A Ar), $78.5\left(\mathrm{CHCHCH}_{2}\right.$ unit A), 72.8 (COCHO unit D), 57.4 $\left(\mathrm{COCHNH}\right.$ unit B), $56.6\left(\mathrm{CHOCH}_{3}\right.$ unit B), $44.2\left(\mathrm{CHCHCHCH}_{2}\right.$ unit $\left.\mathrm{A}\right), 41.2\left(\mathrm{CHCH}_{2} \mathrm{NH}_{\text {unit }} \mathrm{C}\right), 41.0$ $\left(\mathrm{CHCH}_{2} \mathrm{CH}\right.$ unit D), $38.9\left(\mathrm{COCHCH}_{2}\right.$ unit C), $37.9\left(\mathrm{CHCH}_{2} \mathrm{CH}\right.$ unit $\left.\mathrm{A}\right), 36.3\left(\mathrm{CHCH}_{2} \mathrm{C}\right.$ unit B), 25.6 $\left(\left(\mathrm{CH}_{3}\right)_{2} \boldsymbol{C H C H}_{2}\right.$ unit D), $23.2\left(1 \mathrm{x}\left(\boldsymbol{C H}_{3}\right)_{2} \mathrm{CH}\right.$ unit D), $21.6\left(1 \mathrm{x}\left(\boldsymbol{C H}_{3}\right)_{2} \mathrm{CH}\right.$ unit D), $17.8\left(1 \mathrm{x} \boldsymbol{C} \mathrm{H}_{3} \mathrm{CH}\right.$ unit A), 15.1 (1x $\mathrm{CHCH}_{3}$ unit $\mathrm{C}$ ), 11.5, 11.5. HRMS (ESI) calcd for $\mathrm{C}_{34} \mathrm{H}_{44} \mathrm{ClN}_{3} \mathrm{O}_{8}[\mathrm{M}+\mathrm{H}] 658.2890$, found 658.2893 .

\section{General Cloning, Protein Expression, Purification, and Analysis CrpTE and CrpE}

\section{Protein Expression and Purification CrpTE}

Proteins were expressed and purified using a modified protocol. ${ }^{13}$ The $\mathrm{pET}-28 \mathrm{~b}(+)-\mathrm{CrpTE}$ construct was transformed into BL21 (DE3) and grown on LB agar plates supplemented with $50 \mu \mathrm{g} / \mathrm{mL}$ kanamycin at 37 ${ }^{\circ} \mathrm{C}$ overnight. Colonies were picked and grown in $25 \mathrm{~mL} \mathrm{LB}$ broth overnight at $37{ }^{\circ} \mathrm{C}$ supplemented with $50 \mu \mathrm{g} / \mathrm{mL}$ kanamycin. Six liters of TB expression media containing $50 \mu \mathrm{g} / \mathrm{mL}$ of kanamycin were each inoculated with $4 \mathrm{~mL}$ of overnight culture and incubated $\left(37^{\circ} \mathrm{C}, 200 \mathrm{rpm}\right)$ for $\sim 5 \mathrm{~h}$ until the $\mathrm{OD}_{600}$ reached 1.2-1.5. The flasks were removed from the shaker and cooled in an ice bath for 15 minutes (temperature $\sim 20^{\circ} \mathrm{C}$ ), the flasks were returned to shaking, protein expression was initiated with the addition of $100 \mathrm{uM}$ IPTG and proceeded at $20^{\circ} \mathrm{C}$ for 18 hours. Cells were harvested via centrifugation at $6,000 \mathrm{x}$ for $30 \mathrm{~min}$.

The pellet was then re-suspended in cold lysis buffer $(10 \mathrm{~mL} / \mathrm{g}$ cell pellet, $100 \mathrm{mM}$ Sodium Phosphate, 20 $\mathrm{mM}$ imidazole, $300 \mathrm{mM} \mathrm{NaCl}, \mathrm{pH} 8$ ) and treated with $1 \mathrm{mg}$ DNase (Sigma). The solution was placed on ice and subjected to sonication ( $12 \times 10 \mathrm{~s}$ with $50 \mathrm{~s}$ pauses). The suspension was then centrifuged at 50,000 $\mathrm{x} g$ for $30 \mathrm{~min}$ at $4{ }^{\circ} \mathrm{C}$. The supernatant was collected and passed through a $0.45 \mathrm{um}$ filter prior to being loaded onto a $10 \mathrm{~mL}$ NiNTA resin via gravity. The column was washed with 10 column volumes of wash

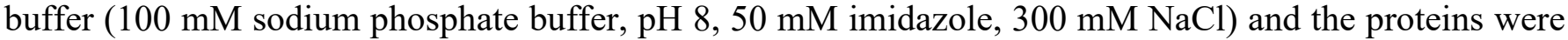
eluted using $15 \mathrm{~mL}$ elution buffer (100 mM sodium phosphate, $\mathrm{pH} 8.0,300 \mathrm{mM}$ imidazole, $300 \mathrm{mM} \mathrm{NaCl})$ via gravity. These were then concentrated to $2.5 \mathrm{~mL}$, subjected to PD-10 buffer exchange/desalting columns (pre equilibrated with storage buffer, $100 \mathrm{mM}$ sodium phosphate, $\mathrm{pH} 7.2,150 \mathrm{mM} \mathrm{NaCl}$ ), flash frozen with liquid nitrogen, and placed in $-80^{\circ} \mathrm{C}$ for storage. Samples were run on a NuPAGE $4-12 \%$ Bis-Tris protein gel to check for purity.

Cloning of CrpE

The C-terminal histidine (His6) tagged crpE gene optimized for the expression in E. coli was synthesized from GenScript (NJ, USA). The CrpE expression construct was designed in pET17b with NdeI and EcoRI restriction sites (pET22b_CrpE).

\section{Protein Expression and Purification of CrpE}

The E. coli strain C43(DE3) was used as a heterologous expression host and the cells were transformed with the expression plasmid pET17b-CrpE. A single ampicillin-resistant colony was inoculated into $50 \mathrm{ml}$ Luria-Bertani (LB) broth containing ampicillin $(100 \mu \mathrm{g}$ per $\mathrm{ml})$ and was grown overnight at $37^{\circ} \mathrm{C}$. The main culture was inoculated with 1:100 dilution of the overnight culture in a terrific broth (TB) medium with the same concentration of ampicillin and incubated at $37^{\circ} \mathrm{C}$ in a baffled flask at $160 \mathrm{rpm}$. When the optical density $\left(\mathrm{A}_{600}\right)$ of the culture reached 0.6 to 0.8 , protein expression was induced with $0.8 \mathrm{mM}$ 
isopropyl- $\beta$-D-thiogalactopyranoside (IPTG). At the same time $0.5 \mathrm{mM}$ of $\delta$-aminolevulinic acid $(\delta$ ALA) and ampicillin ( $50 \mu \mathrm{g}$ per $\mathrm{ml})$ were added. The cells were further grown at lower temperature $\left(28^{\circ} \mathrm{C}\right)$ for 36 hours. The cells were harvested by centrifugation at $5000 \mathrm{rpm}$ for $15 \mathrm{~min}$ at $4{ }^{\circ} \mathrm{C}$ and the cell pellets were stored at $-20^{\circ} \mathrm{C}$.

Purification of CrpE was performed by resuspendeding the pellet in a cold lysis buffer ( $50 \mathrm{mM}$ Tris- $\mathrm{HCl}$, $\mathrm{pH} 7.4$, containing 5\% glycerol, $0.5 \mathrm{mM}$ EDTA, $10 \mathrm{mM} \mathrm{NaCl}$ and $1 \mathrm{mM}$ PMSF). The cells were lysed by ultrasonication (Sonicator, Fisherbrand ${ }^{\mathrm{TM}}$, Fischer Scientific) for $15 \mathrm{~min}$ in an ice bath, followed by the ultracentrifugation step $\left(45,000 \mathrm{rpm}\right.$ at $4^{\circ} \mathrm{C}$ for $\left.35 \mathrm{~min}\right)$. The obtained cell supernatant was loaded on the Ni-NTA column (Qiagen GmbH, Germany) and washed with a buffer (50 mM Tris HCl, pH 7.4, containing $0.1 \mathrm{mM}$ EDTA, $0.1 \mathrm{mM}$ dithioerythritol (DTE) and $5 \mathrm{mM}$ histidine). The His6-tagged CrpE was eluted with the same buffer containing $150 \mathrm{mM}$ histidine. The eluted red fractions were combined and dialyzed against the three exchanges of $1 \mathrm{~L}$ dialysis buffer $(50 \mathrm{mM}$ Tris- $\mathrm{HCl}, \mathrm{pH} 7.4$, containing 0.1 $\mathrm{mM}$ EDTA and $0.1 \mathrm{mM} \beta$-mercaptoethanol) at $4{ }^{\circ} \mathrm{C}$, using a $12-14 \mathrm{~K}$ molecular weight cutoff membrane tube (Spectra Por2 ${ }^{\mathrm{TM}}$, USA). The protein solution was concentrated using $10 \mathrm{~K}$ centricons (Millipore) and aliquoted, flash frozen and stored at $-80^{\circ} \mathrm{C}$ for further use.

The redox partner proteins spinach ferredoxin $(\mathrm{Fdx})$ and ferredoxin reductase $(\mathrm{FdR})$ were expressed and purified as described previously. ${ }^{14,15}$

Spectrophotometric characterization of CrpE

UV-visible spectra of the purified CrpE were recorded at room temperature on a spectrophotometer (Spectra MaxM5, Molecular Devices, USA). The concentration of CrpE was estimated by CO-difference spectra, assuming $\varepsilon(459-490)=91 \mathrm{mM}^{-1} \mathrm{~cm}^{-1}$ as described. ${ }^{16}$ The UV-vis spectra of the purified CrpE showed spectral properties typical for P450 enzymes having the major Soret $(\gamma)$ band at $418 \mathrm{~nm}$, and the smaller $\alpha$ and $\beta$ bands at 567 and 535nm, respectively. The peak maximum at $418 \mathrm{~nm}$ indicates the existence of a protein heme complex in the low spin (LS) state. The spectra of dithionite reduced CrpE showed diminished absorption maxima of the Soret band at $410 \mathrm{~nm}$ and gave a single peak at $550 \mathrm{~nm}$ instead of the $\alpha$ and $\beta$ bands (Supplementary Figure 1). The spectra of the carbon monoxide bound forms of CrpE exhibited atypical peak with a maximum at $448 \mathrm{~nm}$, suggesting native thiolated (cysteinate coordinated) FeII-CO complexes of P450s (supplementary Figure 1).

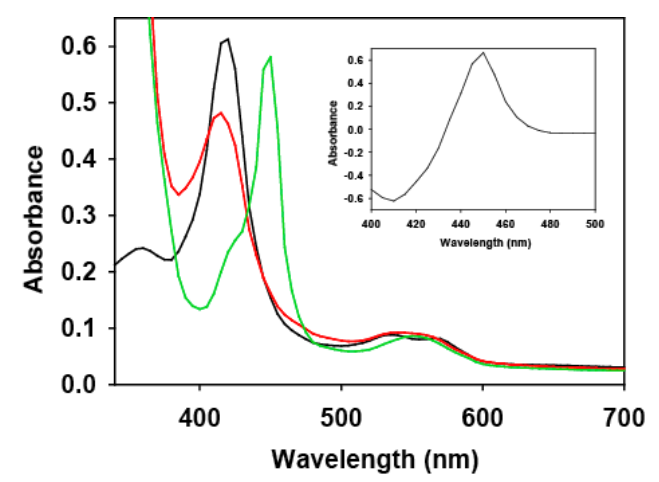

Supplemental Figure 1: Spectroscopic characterization of CrpE. The oxidized (black line), dithionite reduced (red line) and the reduced CO-complexed (green line), forms of CrpE are shown. The baseline was recorded between 200 to $700 \mathrm{~nm}$. The UV-vis spectra of $\mathrm{CrpE}(6.5 \mu \mathrm{M})$ was recorded in $50 \mathrm{mM}$ Tris- $\mathrm{HCl}$ buffer, $\mathrm{pH} 7.4$, containing $2 \%$ glycerol. The inset shows the COcomplexed form of CrpE with a peak maximum at $448 \mathrm{~nm}$.

\section{Spin-state shift and substrate dissociation constant determination}

The spin-state shifts upon substrate binding were assayed at room temperature under aerobic condition using a SpectraMax M5 UV-visible spectrophotometer (Molecular Devices) as described. ${ }^{17}$ Briefly, the test 
compound stocks $(5 \mathrm{mM})$ were prepared in DMSO. Serial dilutions of the stocks were prepared to achieve target concentrations of the stock solutions used for binding assays. The ligands were titrated in small $(<1$ $\mu \mathrm{L})$ aliquots into the solution of the $\mathrm{P} 450(1.5 \mu \mathrm{M})$ in $50 \mathrm{mM}$ potassium phosphate buffer, $\mathrm{pH} 7.4$, containing $2 \%$ glycerol $0.2 \mathrm{mM}$ EDTA and $0.4 \mathrm{mM}$ DTT, with the starting spectra subtracted from the subsequent traces. The same amounts of DMSO alone were added to the protein in the reference cuvette. The spectral changes between $360 \mathrm{~nm}$ and $500 \mathrm{~nm}$ were recorded until the P450 CrpE became saturated. The dissociation constant $\left(\mathrm{K}_{\mathrm{D}}\right)$ for the CrpE with the native substrate and the analogous was calculated by fitting the peakto-trough difference against the substrate concentration to either a rectangular hyperbolic function $(\mathrm{Kd}>5$ $\mathrm{x}[\mathrm{P} 450])$ or a quadratic function for high-affinity ligands $(\mathrm{Kd} \leq 5 \mathrm{x}$ [P450]). A nonlinear tight binding quadratic equation, ${ }^{18}$ which accounts the quantity of the substrate consumed in complex with the P450 in determining the $\mathrm{K}_{\mathrm{D}}$ value for the substrates. The relevant equation is : $\Delta \mathrm{A}=(\mathrm{Amax} / 2[\mathrm{E}])\{(\mathrm{KD}+[\mathrm{E}]+$ $[\mathrm{S}])-\{(\mathrm{KD}+[\mathrm{E}]+[\mathrm{S}]) 2-4[\mathrm{E}][\mathrm{S}]\} 1 / 2\}$, where $\Delta \mathrm{A}$ represents the observed peak peak-to-trough absorbance difference at each substrate addition, Amax is the maximum absorbance difference at substrate saturation, $[\mathrm{E}]$ is the total enzyme concentration and $[\mathrm{S}]$ is the substrate concentration. The data fitting was performed using Origin 8.1 software. All titrations were done for three times and the $\mathrm{K}_{\mathrm{D}}$ values reported are the mean for the three sets of the experimental data.
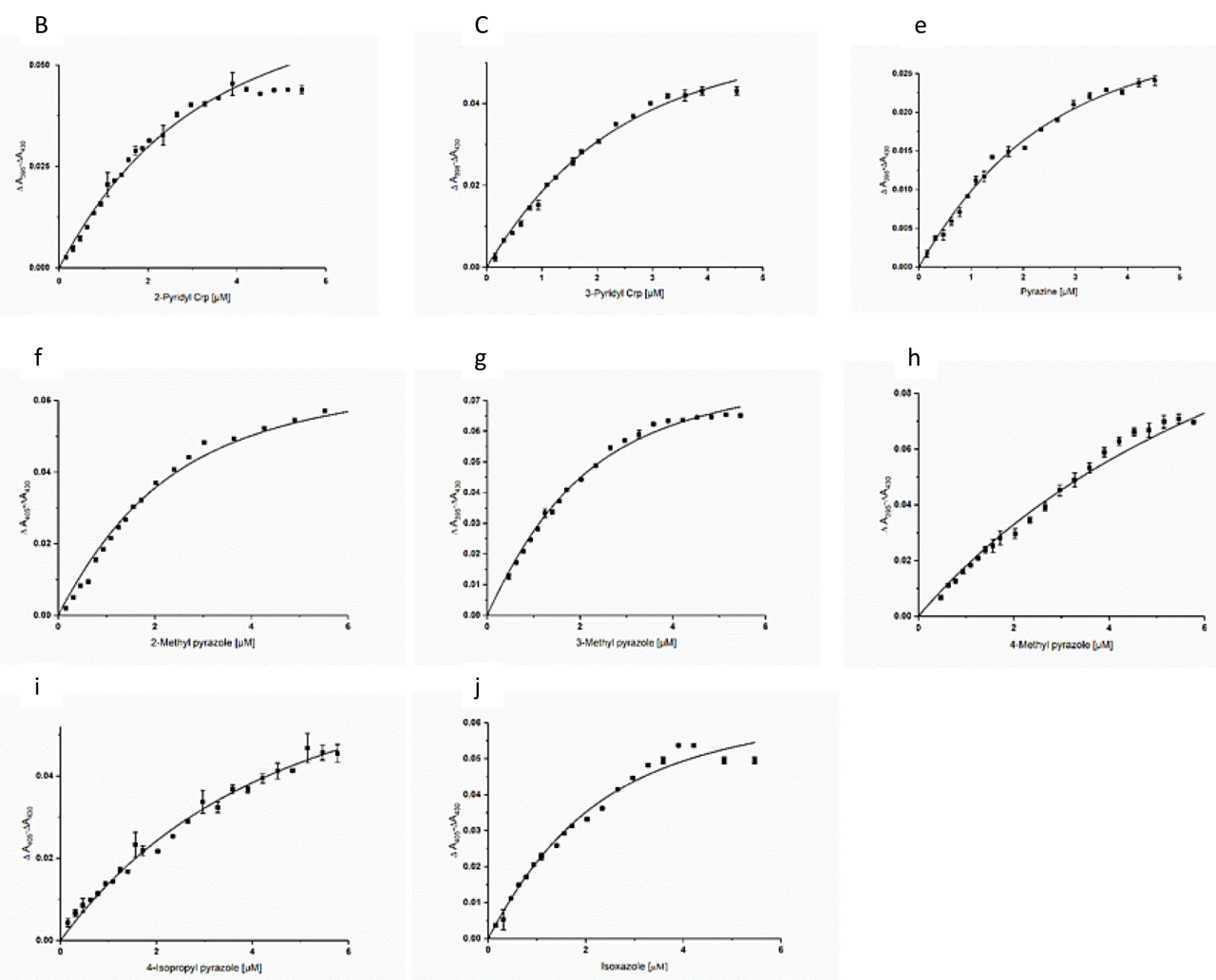

Supplementary Figure 2: Type I shift induced by binding of A) 2-pyridyl Crp B) 3-pyridyl Crp C) Pyrazine D) 2.methyl pyrazole (2MP) E) 3-methyl pyrazole (3MP) F) 4-methyl pyrazole (4MP) G) 4-isopropyl pyrazole and H) isoxazole to the P450 CrpE. 


\section{Analytical Evaluation of Cryptophycin Analogs with CrpE and CrpTE}

Analytical Characterization of CrpTE

Analytical scale reactions were carried out under the following conditions: To a $1.5 \mathrm{~mL}$ Eppendorf tube was added phosphate buffer ( $\mathrm{pH}=7.2,100 \mathrm{mM}, 300 \mathrm{uL}$ ), DMSO (to 7\% total concentration) and seco substrate $(75 \mu \mathrm{M})$ suspended in DMSO. This was then treated with CrpTE enzyme (in phosphate buffer $\mathrm{pH}$ $=7.2100 \mathrm{mM}, 0.5 \mu \mathrm{M}$ ) and shaken at $30^{\circ} \mathrm{C}$ for 12 hours. Upon completion of the reaction, the aqueous layer was extracted with $0.300 \mathrm{~mL}$ of Ethyl Acetate, $150 \mathrm{uL}$ was removed, dried directly into HPLC vials, and resuspended in $70 \mathrm{uL}$ of Methanol for LCMS analysis. LCMS analysis was carried out on an Agilent TOF HPLC-MS (Life Sciences Institute, University of Michigan) equipped with a high-resolution electrospray mass spectrometery (ESI-MS) (wavelength set to $235 \mathrm{~nm}$ ) using a Phenomenex Synergi HydroRP C18 column (100 x $2.00 \mathrm{~mm}, 4$ micron). HPLC conditions were as follows: mobile phase $(\mathrm{A}=$ deionized water $+0.1 \%$ formic acid, $\mathrm{B}=95 \%$ acetonitrile/deionized water $+0.1 \%$ formic acid); $30 \% \mathrm{~B}$ for 2 minutes, $30 \%$ to $70 \%$ B over 17 minutes, $100 \%$ B for 3 minutes; $0.3 \mathrm{~mL} / \mathrm{min}$; injection volume $2 \mu \mathrm{L}$, retention times and observed $\mathrm{m} / \mathrm{z}$ values are seen in table 1 . Analysis of data for $\%$ conversion, TTN (calculated for product), and cyclization:hydrolysis were evaluated using the areas of the HPLC peak corresponding to starting material, hydrolysis, and products. The extinction coefficient of each of the given peaks was essentially identical given that the predominant absorbance is caused by the two aryl aryl rings.

$$
\mathrm{TTN}=\left(\frac{\text { AREA }_{\text {Product }}}{\text { AREA }_{\text {Product }}+\mathrm{AREA}_{\text {Hydrolysis }}+\mathrm{AREA}_{\text {Starting Material }}}\right)\left(\frac{\left[\text { Subsrate }_{\text {Total }}\right.}{[\mathrm{TE}]_{\text {Total }}}\right)
$$

Analytical Conversion Analysis of CrpE

The in vitro conversions of cryptophycin 3 and its analogues were performed using $\operatorname{CrpE}(0.5 \mu \mathrm{M})$, spinach Fdx $(10 \mu \mathrm{M}), \mathrm{FdR}(1.5 \mu \mathrm{M}), \mathrm{MgCl}_{2}(1 \mathrm{mM})$ [P450: Fdx: FdR of 1: 20: 3 ratio] and a cofactor regenerating system containing glucose-6-phosphate $(5 \mathrm{mM})$ and glucose-6-phosphate dehydrogenase (1 $\mathrm{U})$ in $20 \mathrm{mM}$ potassium phosphate buffer, $\mathrm{pH} 7.4$ containing $2 \%$ glycerol. The total reaction volume was $250 \mu \mathrm{l}$. The substrate was dissolved in DMSO and added to a final concentration of $50 \mu \mathrm{M}$. The reactions were initiated by adding NADPH $(500 \mu \mathrm{M})$, incubated for $30 \mathrm{~min}$ at $30^{\circ} \mathrm{C}$, and terminated by the addition of $500 \mu \mathrm{l}$ of chloroform. The samples were vigorously mixed, centrifuged and the organic layer was extracted twice with chloroform. The pooled samples were dried in the nitrogen stream and analyzed via Agilent TOF as described below. All experiments were performed by using the same batch of the purified proteins. A negative control with no P450 added was used to verify the P450-dependent reactions.

\section{LCMS Analysis of Analytical CrpE Conversion}

LCMS analysis was carried out on an Agilent TOF HPLC-MS (Life Sciences Institute, University of Michigan) equipped with a high-resolution electrospray mass spectrometer (ESI-MS) (wavelength set to $235 \mathrm{~nm}$ ) using a Phenomenex Synergi HydroRP C18 column (100 x 2.00 mm, 4 micron). HPLC conditions were as follows: mobile phase $(\mathrm{A}=$ deionized water $+0.1 \%$ formic acid, $\mathrm{B}=95 \%$ acetonitrile/deionized water $+0.1 \%$ formic acid); $30 \% \mathrm{~B}$ for 2 minutes, $30 \%$ to $70 \% \mathrm{~B}$ over 17 minutes, $100 \%$ B for 3 minutes; $0.3 \mathrm{~mL} / \mathrm{min}$; injection volume $2 \mu \mathrm{L}$, retention times and observed $\mathrm{m} / \mathrm{z}$ values are seen below. 

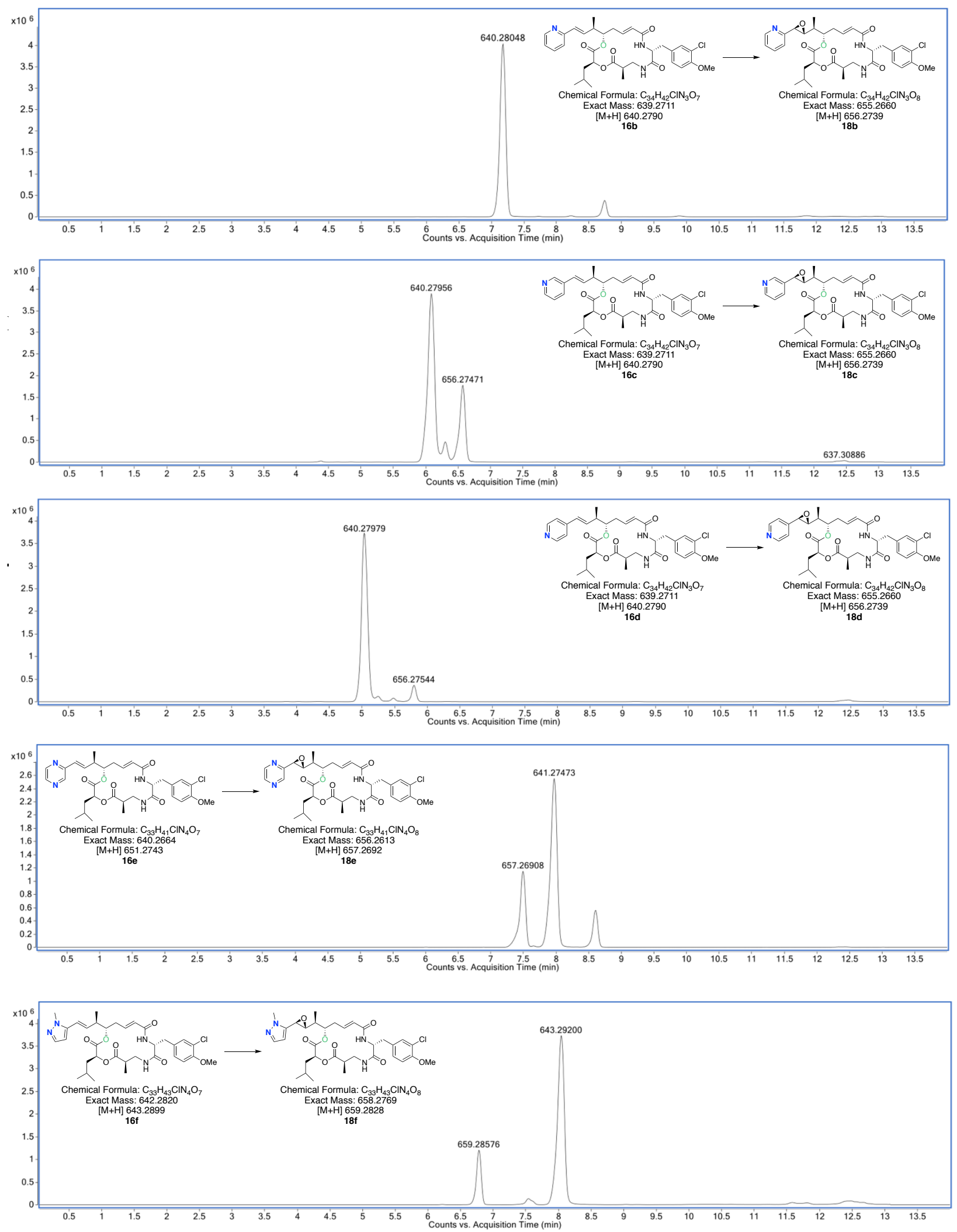

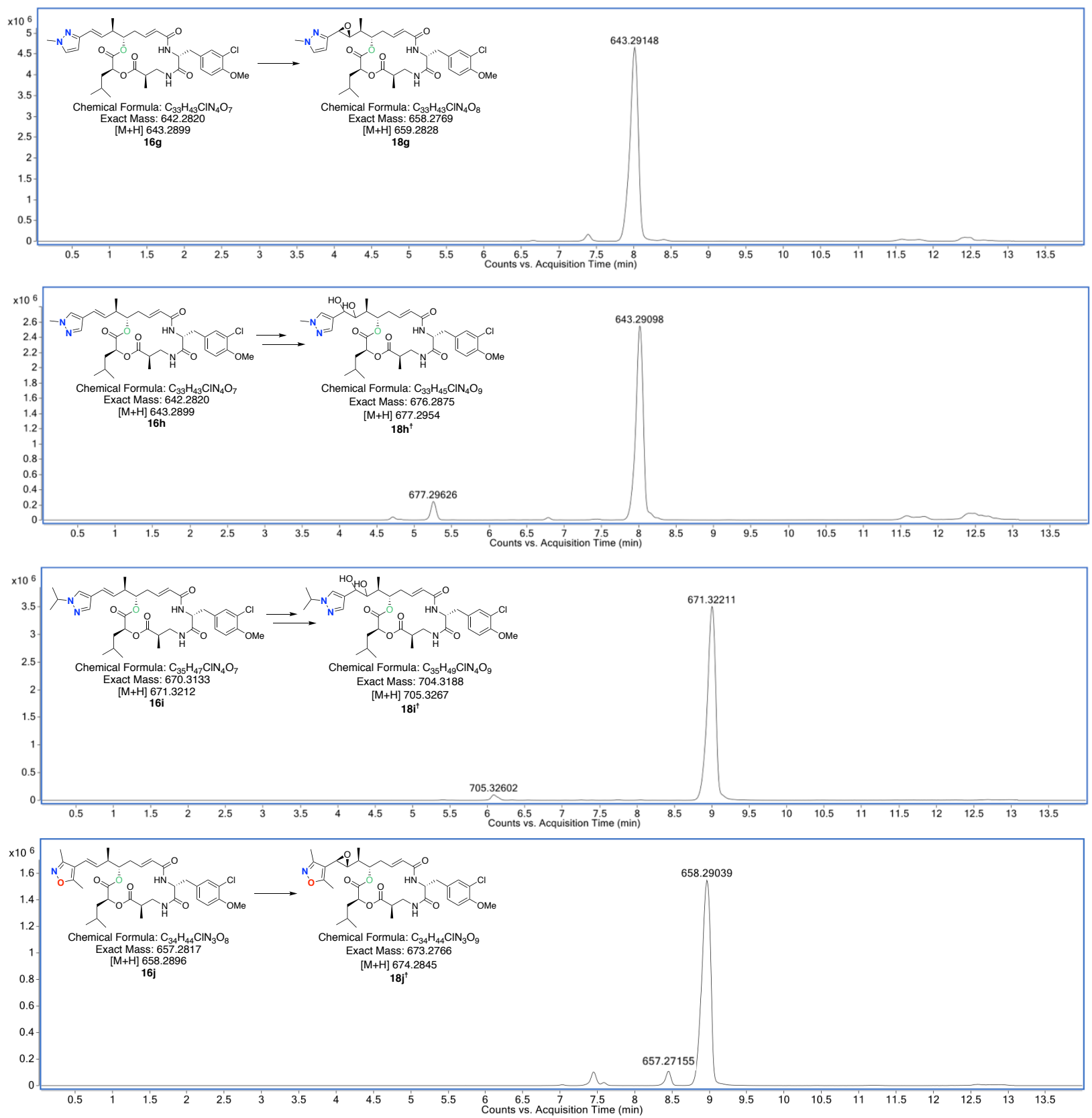

Supplemental Figure 3: TOF-LCMS chromatogram analysis, for CrpE reactions (UV chromatograms at $235 \mathrm{~nm}$ ) 
Supplemental Table 1: In Vitro disk diffusion assay results

\begin{tabular}{|c|c|c|c|c|c|c|c|c|c|c|c|c|}
\hline & & L1210 & Col38 & CFU-GM & H116 & H125 & OVC-5 & U251N & MCF-7 & LNCaP & PANC-1 & CEM \\
\hline DMSO & & 0 & 0 & 0 & 0 & 0 & 0 & 0 & 0 & 0 & 0 & 0 \\
\hline 2 pyridyl & $1.9 \mathrm{mg} / \mathrm{ml}$ & 500 & 700 & 500 & 500 & 600 & 650 & 700 & 800 & 850 & 600 & 500 \\
\hline " & $1 / 4$ & 450 & 500 & 500 & 500 & 500 & 600 & 650 & 800 & 800 & 650 & 500 \\
\hline$"$ & $1 / 16$ & 400 & 250 & 450 & 400 & 500 & 500 & 500 & 600 & 650 & 550 & 450 \\
\hline 3 pyridyl & $2.4 \mathrm{mg} / \mathrm{ml}$ & & & & & & 800 & & & & & \\
\hline " & $1 / 4$ & & & & & & 750 & & & & & \\
\hline$"$ & $1 / 16$ & 800 & 700 & 700 & 800 & 850 & 700 & 800 & 500 & 800 & 800 & 600 \\
\hline$"$ & $1 / 64$ & 650 & 650 & 600 & 650 & 800 & 650 & 600 & 450 & 800 & 750 & 600 \\
\hline$"$ & $1 / 256$ & 550 & 500 & 500 & 500 & 600 & & 600 & 400 & 700 & 600 & 350 \\
\hline 4 pyridyl & $2 \mathrm{mg} / \mathrm{ml}$ & & & & & & 800 & & & & & \\
\hline " & $1 / 4$ & & & & & & 750 & & & & & \\
\hline$"$ & $1 / 16$ & 700 & 800 & 700 & 700 & 1000 & 750 & 750 & 550 & 850 & 800 & 750 \\
\hline$"$ & $1 / 64$ & 600 & 700 & 550 & 600 & 800 & 750 & 600 & 450 & 800 & 750 & 650 \\
\hline$"$ & $1 / 256$ & 550 & 500 & 450 & 500 & 650 & 600 & 600 & 400 & 700 & 600 & 600 \\
\hline pyrazine & $2.2 \mathrm{mg} / \mathrm{ml}$ & & & & & & 750 & & & & & \\
\hline " & $1 / 4$ & & & & & & 700 & & & & & \\
\hline$"$ & $1 / 16$ & 700 & 700 & 550 & 600 & 750 & 650 & 750 & 500 & 800 & 750 & 750 \\
\hline$"$ & $1 / 64$ & 550 & 450 & 500 & 400 & 600 & & 600 & 400 & 750 & 700 & 600 \\
\hline$"$ & $1 / 256$ & 300 & 200 & 350 & 300 & 450 & & 550 & 300 & 500 & 600 & 450 \\
\hline 2 methyl pyrazole & $2.6 \mathrm{mg} / \mathrm{ml}$ & & & & & & & & $>1000$ & & & \\
\hline " & $1 / 4$ & & & & & & & & $>1000$ & & & \\
\hline$"$ & $1 / 16$ & & & & & & & & $>1000$ & & & 850 \\
\hline$"$ & $1 / 64$ & 550 & 750 & 600 & 550 & 500 & $>1000$ & 900 & 800 & 900 & 700 & 600 \\
\hline$"$ & $1 / 256$ & 400 & 500 & 500 & 350 & 450 & 850 & 800 & 600 & 700 & 600 & 500 \\
\hline$"$ & $1 / 1024$ & 300 & 300 & 400 & 300 & 250 & 650 & 550 & 400 & 500 & 500 & 350 \\
\hline \multicolumn{13}{|l|}{3 methyl pyrazole } \\
\hline " & $1 / 4$ & & & & & & & & & & $>1000$ & \\
\hline$"$ & $1 / 16$ & & & 500 & 600 & 650 & 750 & 850 & 800 & 900 & 900 & 800 \\
\hline$"$ & $1 / 64$ & & & 300 & 400 & 500 & 550 & 750 & 650 & 700 & 700 & 550 \\
\hline$"$ & $1 / 256$ & & & 100 & 250 & 250 & 300 & 400 & 400 & 500 & 500 & 400 \\
\hline$"$ & $1 / 1,024$ & & & 0 & 100 & 150 & 100 & 200 & 200 & 200 & 200 & 200 \\
\hline 4 methyl pyrazole & $2.8 \mathrm{mg} / \mathrm{ml}$ & & & & & & 800 & & & & & \\
\hline " & $1 / 4$ & & & & & & 800 & & & & & \\
\hline$"$ & $1 / 16$ & 350 & 800 & 400 & 450 & 850 & 900 & 800 & 550 & 1000 & 850 & 700 \\
\hline$"$ & $1 / 64$ & 250 & 650 & 200 & 350 & 750 & 600 & 750 & 500 & 800 & 750 & 700 \\
\hline$"$ & $1 / 256$ & 100 & 500 & 150 & 200 & 750 & 600 & 700 & 450 & 750 & 700 & 550 \\
\hline " & $1 / 1024$ & & & & & & & & & 700 & & 300 \\
\hline Isopropyl pyrazole & $2.6 \mathrm{mg} / \mathrm{ml}$ & 600 & 850 & 550 & 600 & 550 & 800 & 750 & 800 & 900 & 700 & 450 \\
\hline " & $1 / 4$ & 550 & 800 & 600 & 550 & 550 & 600 & 550 & 600 & 800 & 600 & 450 \\
\hline " & $1 / 16$ & 500 & 600 & 500 & 500 & 450 & 600 & 550 & 550 & 700 & 550 & 450 \\
\hline Isoxazole & $2.5 \mathrm{mg} / \mathrm{ml}$ & 200 & 400 & 250 & 300 & 350 & 400 & 400 & 500 & 500 & 400 & 350 \\
\hline " & $1 / 4$ & 200 & 250 & 250 & 250 & 300 & 500 & 500 & & & 500 & 300 \\
\hline " & $1 / 16$ & & 200 & & & 150 & 250 & 200 & 300 & 400 & 600 & 350 \\
\hline \multicolumn{13}{|l|}{3 pyr dimethyl } \\
\hline " & $1 / 4$ & & & 800 & 750 & 800 & 800 & 900 & 750 & 800 & 800 & 700 \\
\hline$"$ & $1 / 16$ & & & 650 & 650 & 800 & 700 & 800 & 800 & 700 & 600 & 700 \\
\hline$"$ & $1 / 64$ & & & 500 & 550 & 600 & 600 & 700 & 600 & 600 & 600 & 600 \\
\hline$"$ & $1 / 256$ & & & 350 & 500 & 600 & 500 & 600 & 500 & 550 & 450 & 500 \\
\hline$"$ & $1 / 1,024$ & & & 200 & 300 & 400 & 400 & 450 & 400 & 400 & 400 & 400 \\
\hline 4 pyr dimethyl & & & & & & & & & & & 900 & \\
\hline " & $1 / 4$ & & & & & & & & & & 800 & 750 \\
\hline$"$ & $1 / 16$ & & & 650 & 750 & 800 & 650 & 850 & 850 & 800 & 750 & 600 \\
\hline$"$ & $1 / 64$ & & & 500 & 550 & 650 & 600 & 700 & 800 & 600 & 600 & 600 \\
\hline$"$ & $1 / 256$ & & & 400 & 500 & 600 & 500 & 600 & 600 & 600 & 600 & 500 \\
\hline$"$ & $1 / 1,024$ & & & 300 & 350 & 400 & 400 & 500 & 450 & 500 & 500 & 400 \\
\hline \multicolumn{13}{|l|}{ 4MP-dimethyl } \\
\hline$"$ & $1 / 4$ & & & 500 & 800 & 850 & 800 & 900 & 800 & 800 & 800 & 800 \\
\hline$"$ & $1 / 16$ & & & 200 & 800 & 800 & 450 & 600 & 700 & 600 & 400 & 650 \\
\hline$"$ & $1 / 64$ & & & 100 & 750 & 750 & 300 & 450 & 250 & 500 & 50 & 600 \\
\hline$"$ & $1 / 256$ & & & 0 & 600 & 600 & 100 & 300 & 450 & 400 & 200 & 550 \\
\hline$"$ & $1 / 1,024$ & & & & & & & 0 & 300 & 150 & 200 & 500 \\
\hline
\end{tabular}




\section{${ }^{1} \mathrm{H}$ and ${ }^{13} \mathrm{C}$ Spectra}<smiles></smiles>

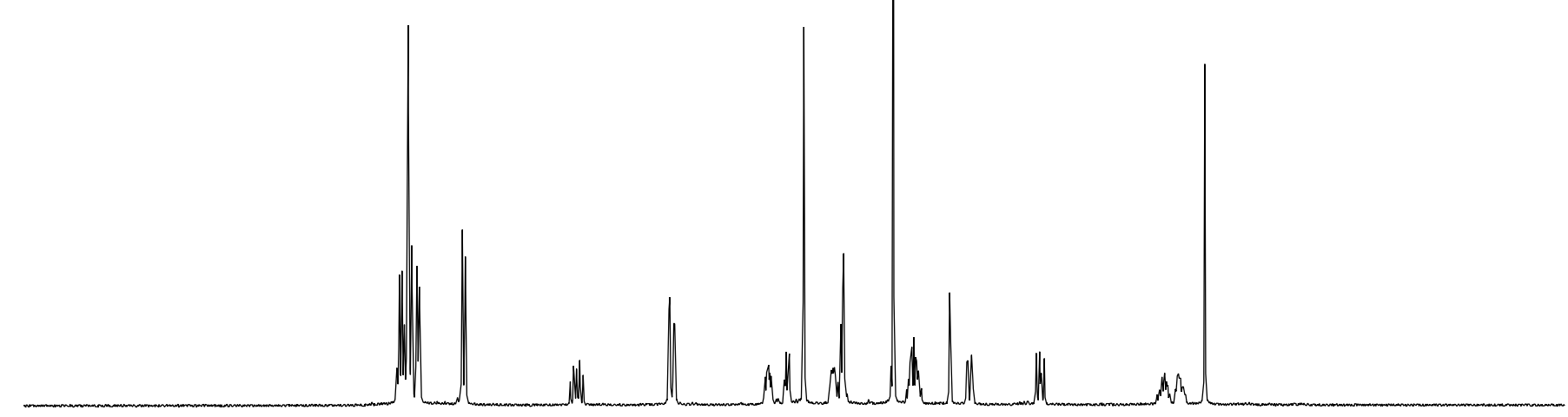

$\begin{array}{lllllllllllllllllllll}1.0 & 9.5 & 9.0 & 8.5 & 8.0 & 7.5 & 7.0 & 6.5 & 6.0 & 5.5 & \begin{array}{c}5.0 \\ \text { Chemical Shift(ppm) }\end{array} & \begin{array}{l}4.5 \\ 4.0\end{array} & 3.0 & 2.5 & 2.0 & 1.5 & 1.0 & 0.5 & 0.0 & -0.5 & -1\end{array}$
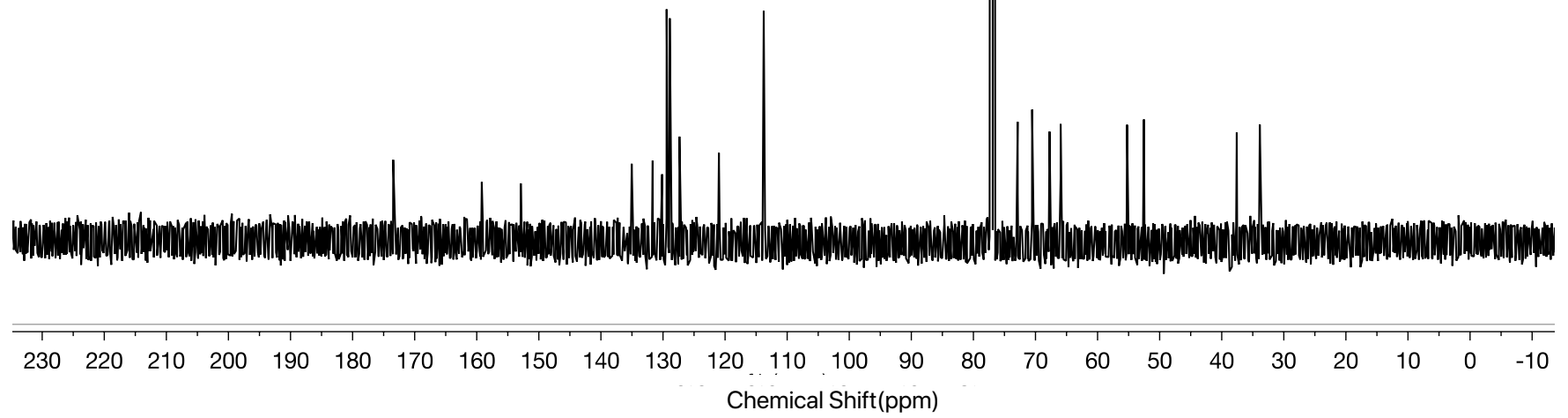


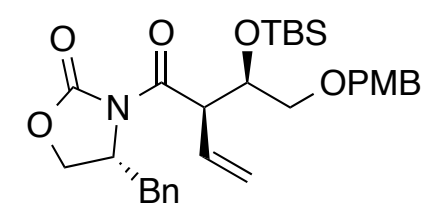
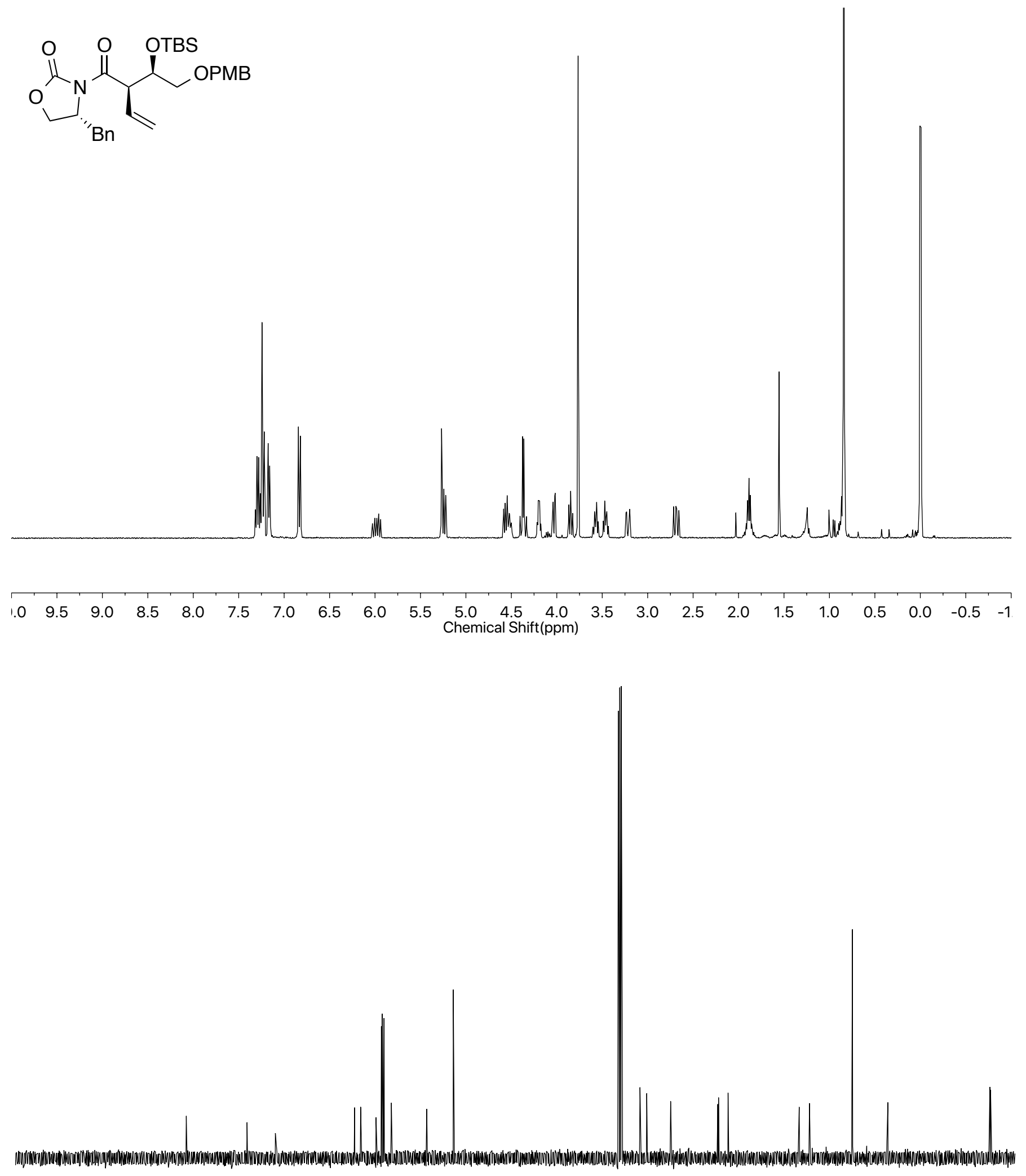

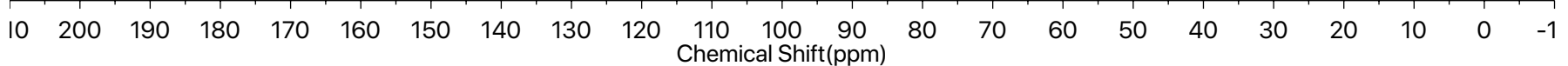


<smiles>[R5][R15]([H])([H])OCC[C@@H]([OH2+])[C@H](C=C)CO</smiles>

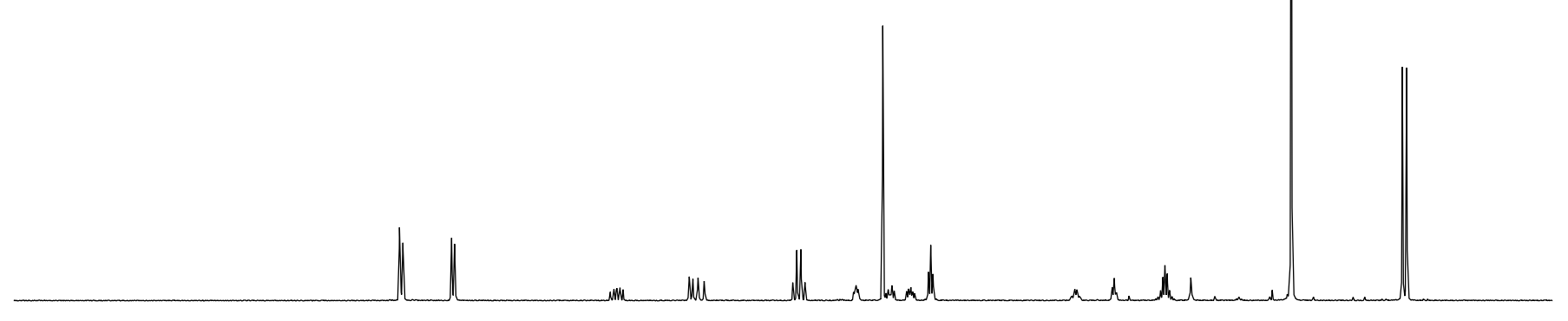

$\begin{array}{lllllllllllllllllllll}1.0 & 9.5 & 9.0 & 8.5 & 8.0 & 7.5 & 7.0 & 6.5 & 6.0 & 5.5 & \begin{array}{c}5.0 \\ \text { Chemical Shift(ppm) }\end{array} & 4.5 & 3.0 & 2.5 & 2.0 & 1.5 & 1.0 & 0.5 & 0.0 & -0.5 & -1\end{array}$

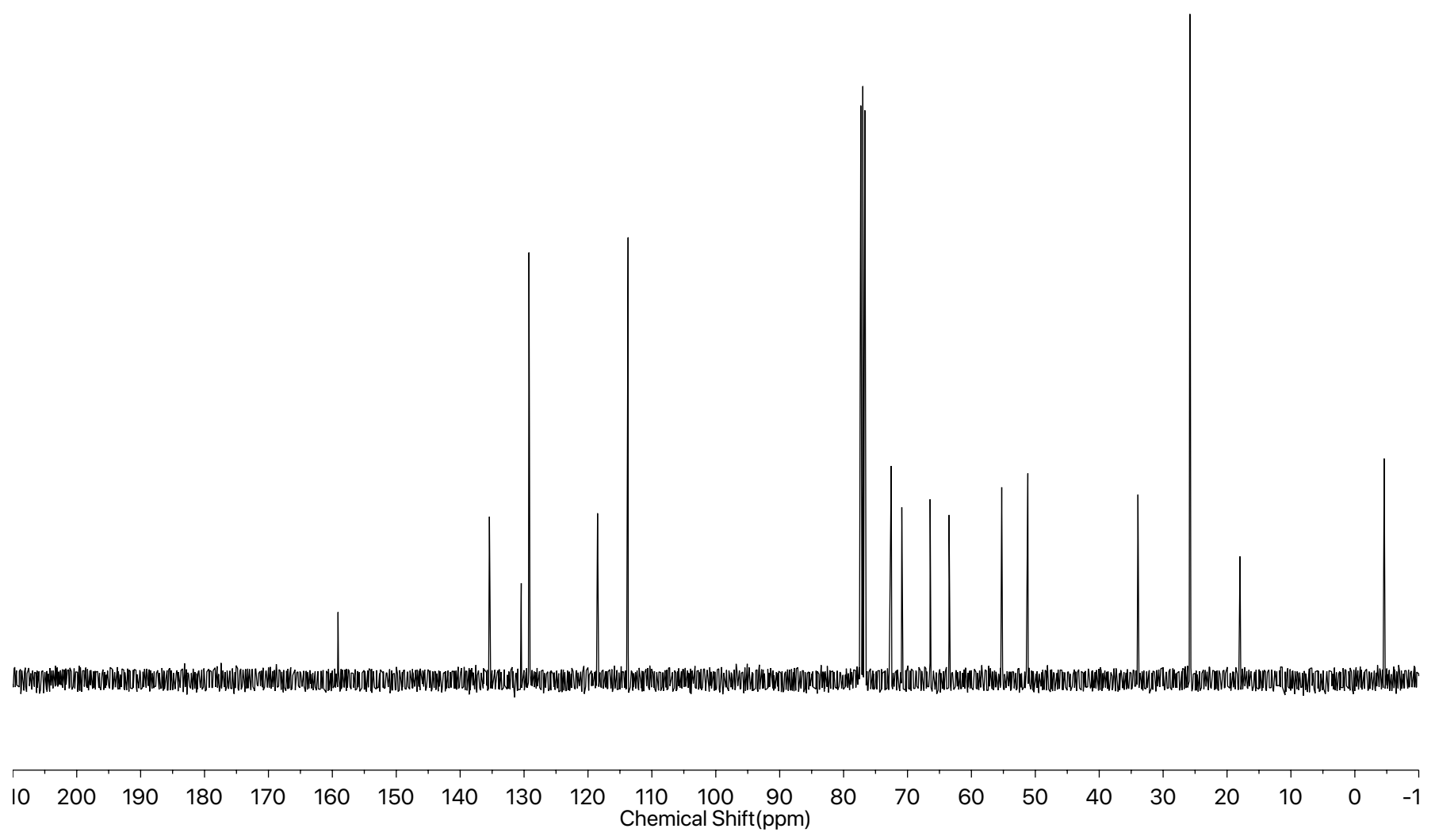




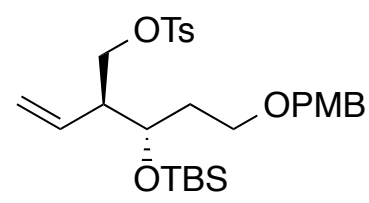

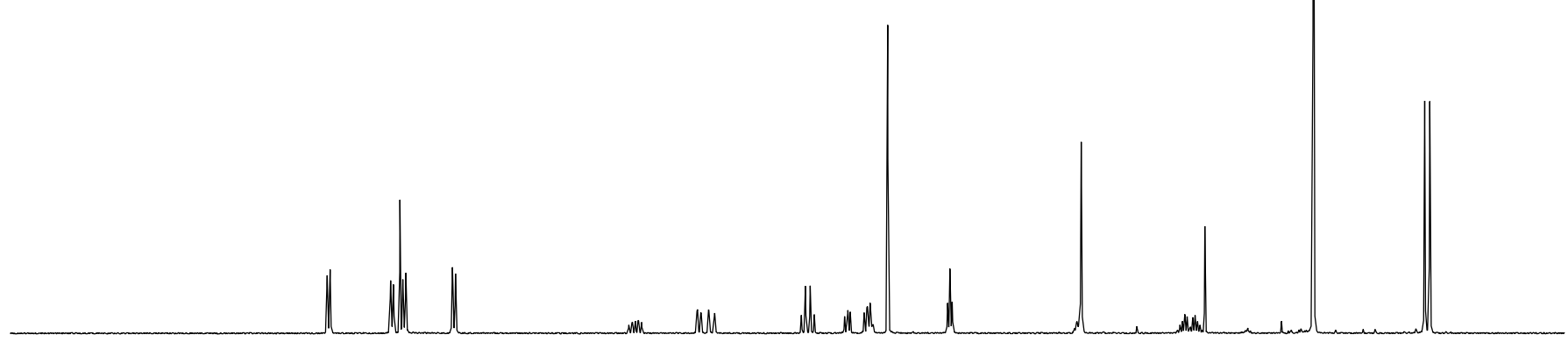

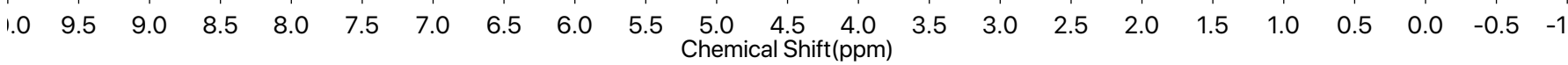
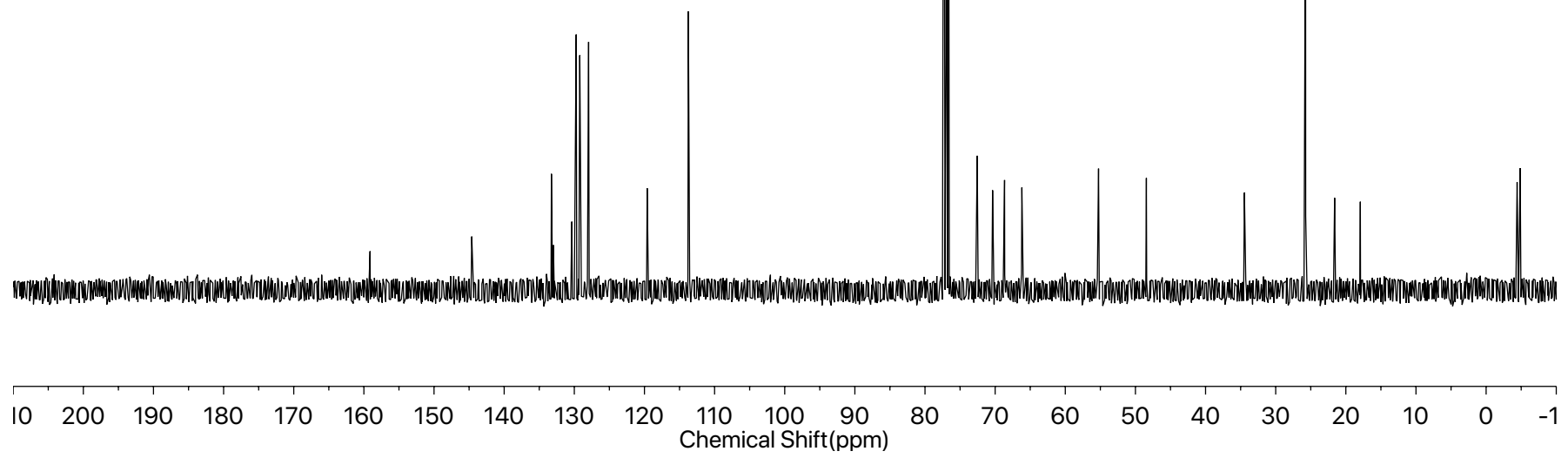
<smiles>C=CC(C)C(C[SeH3])CCO[18OH]</smiles>
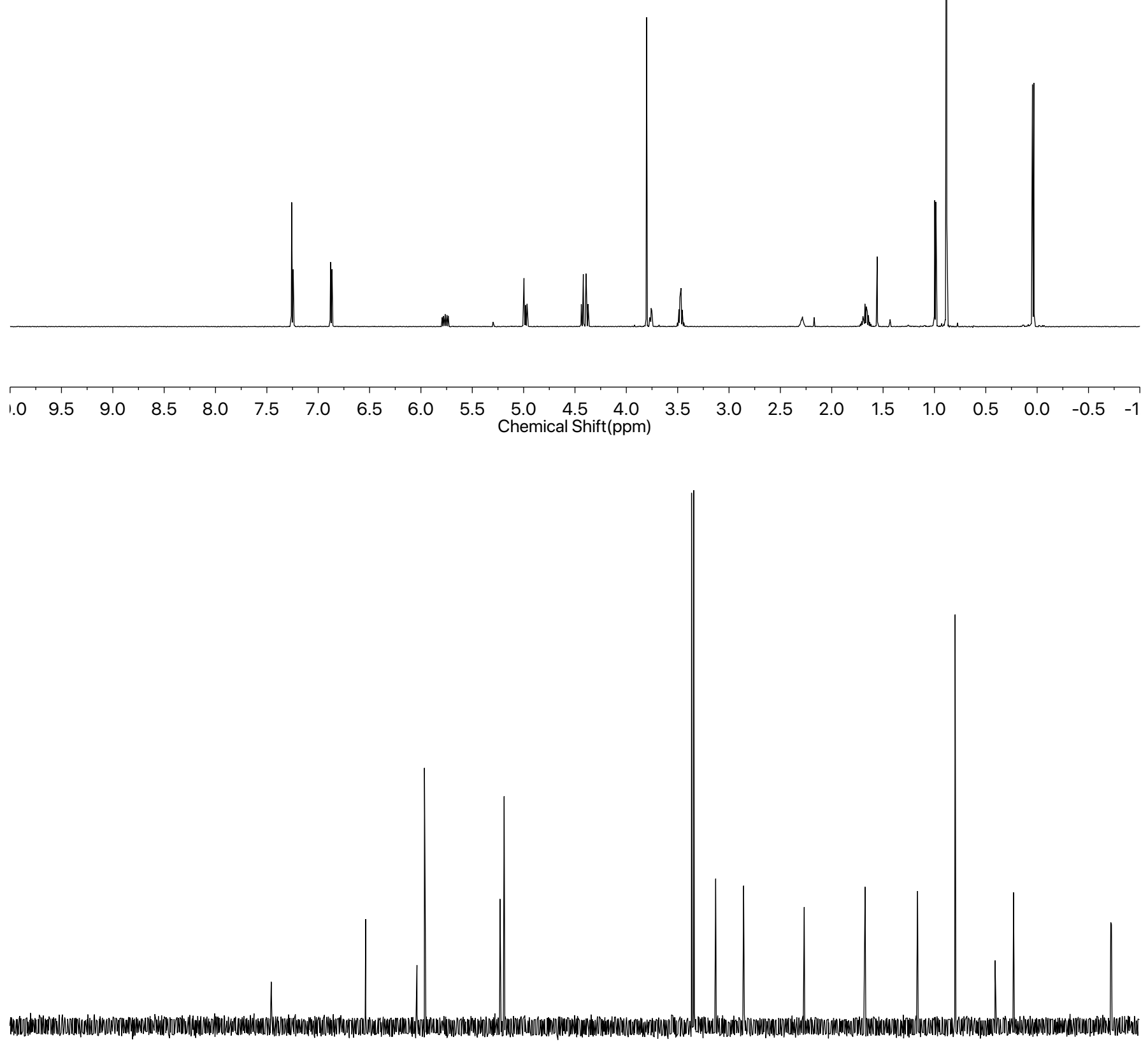

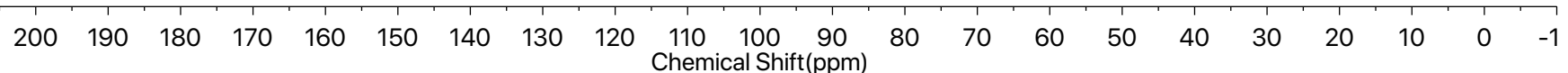




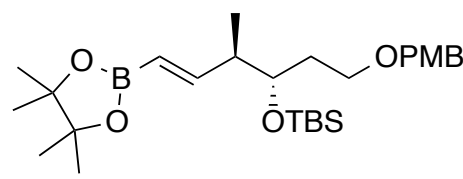

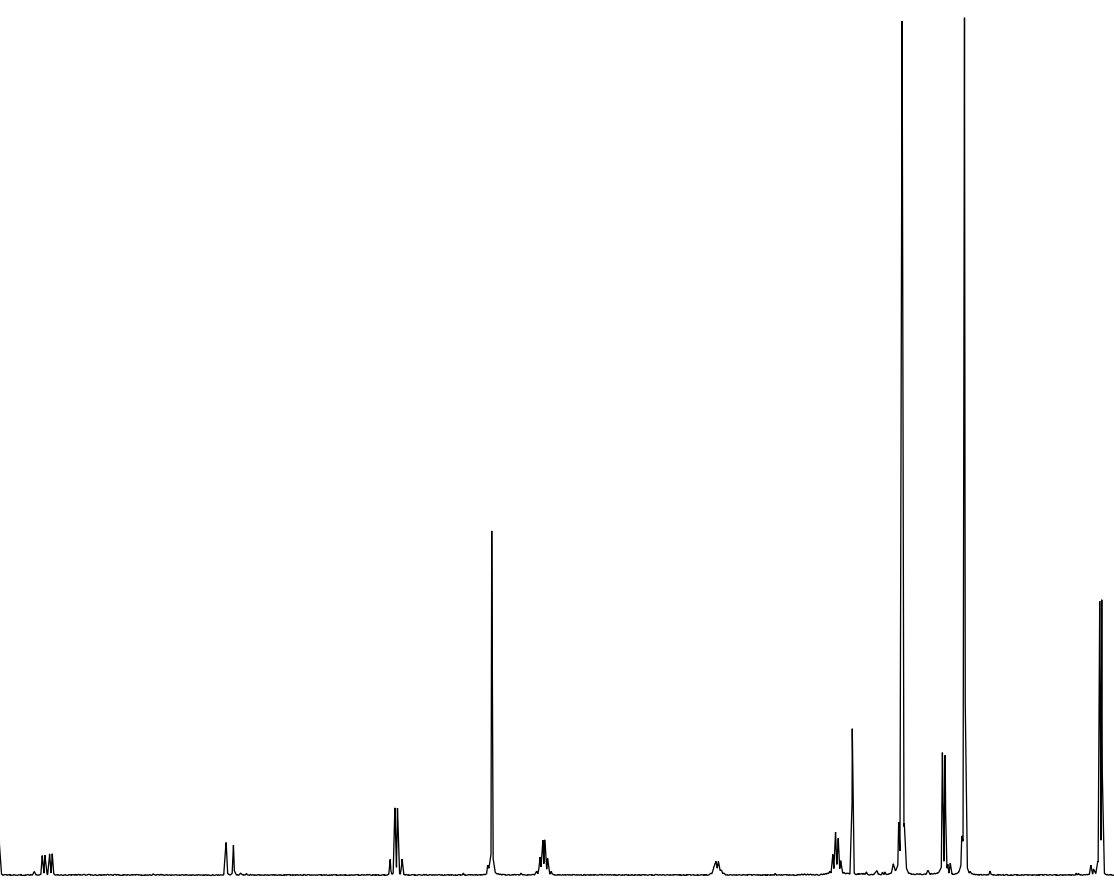

$\begin{array}{llllllllllllllllllllll}1.0 & 9.5 & 9.0 & 8.5 & 8.0 & 7.5 & 7.0 & 6.5 & 6.0 & 5.5 & \begin{array}{c}5.0 \\ \text { Chemical Shift(ppm) }\end{array} & \begin{array}{l}4.5 \\ \text { (p.5 }\end{array} & 3.0 & 2.5 & 2.0 & 1.5 & 1.0 & 0.5 & 0.0 & -0.5 & -1\end{array}$

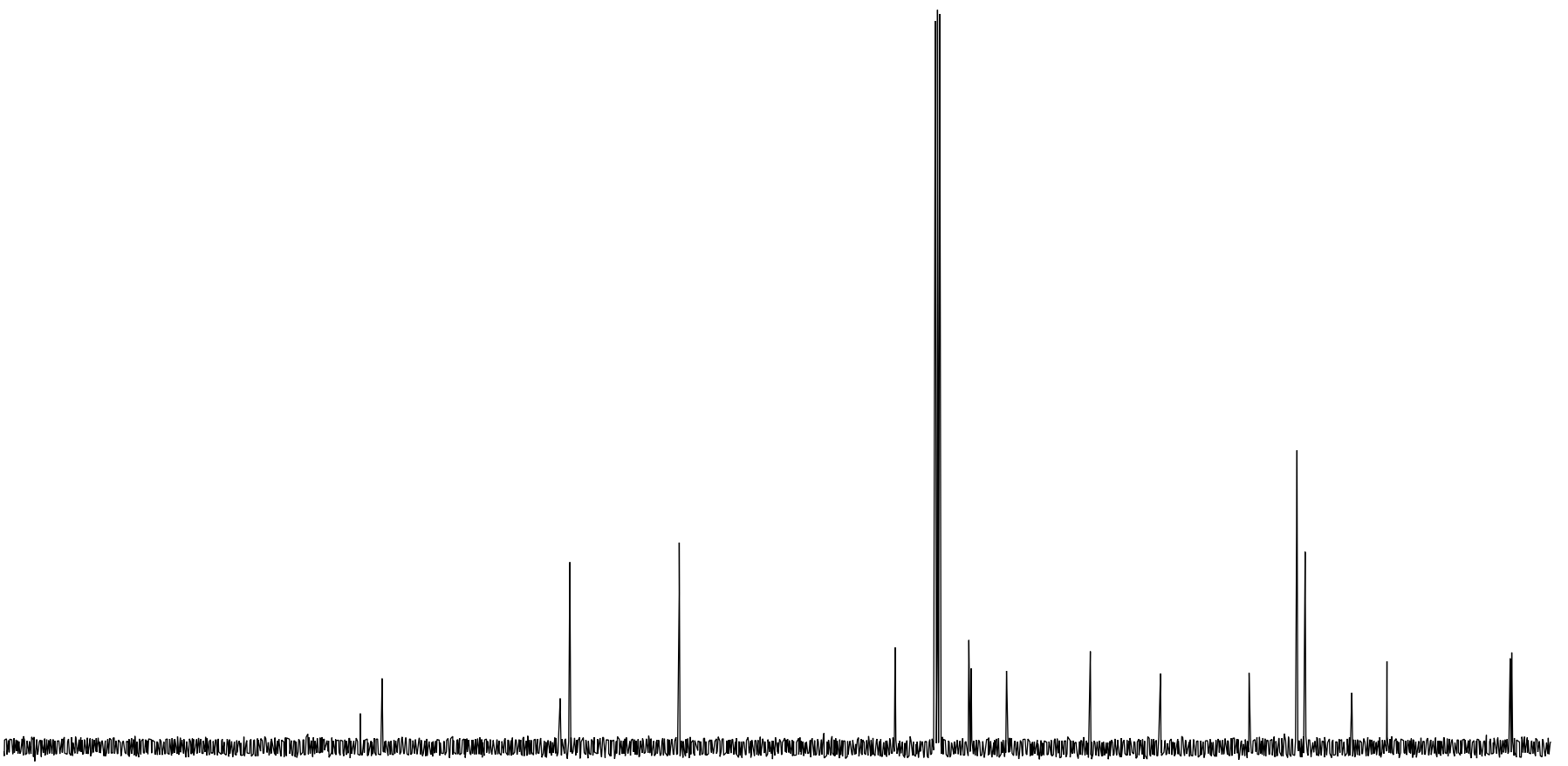

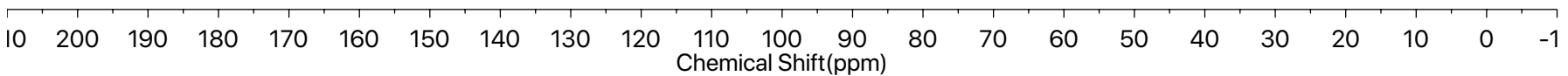


<smiles>CC(C=CB1OC(C)(C)C(C)(C)O1)C([OH+])CCO</smiles>

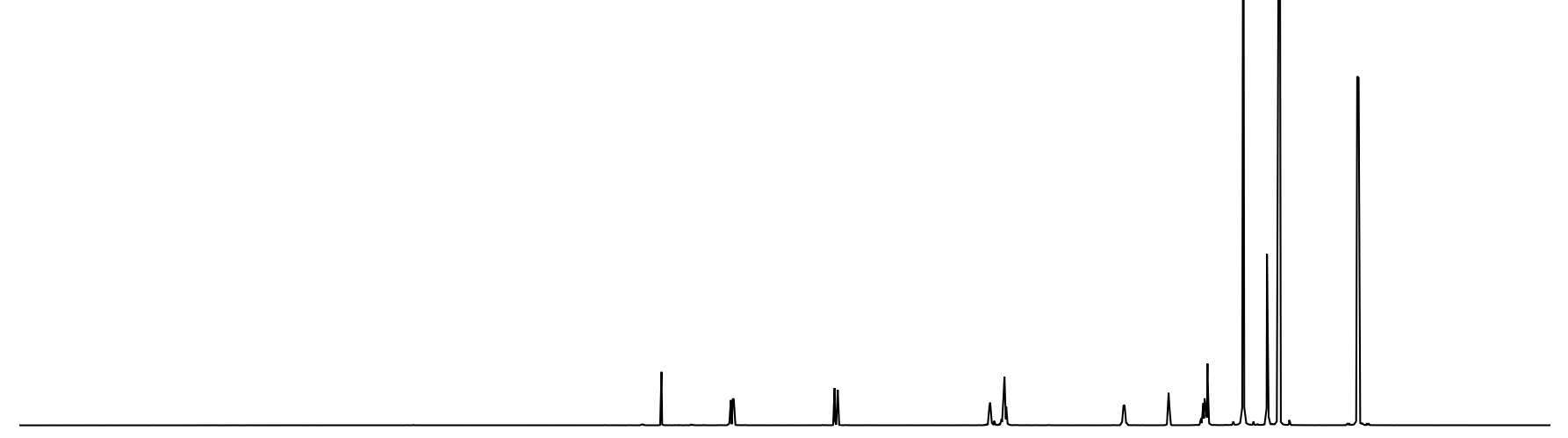

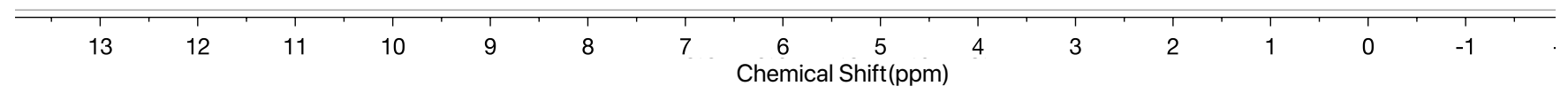
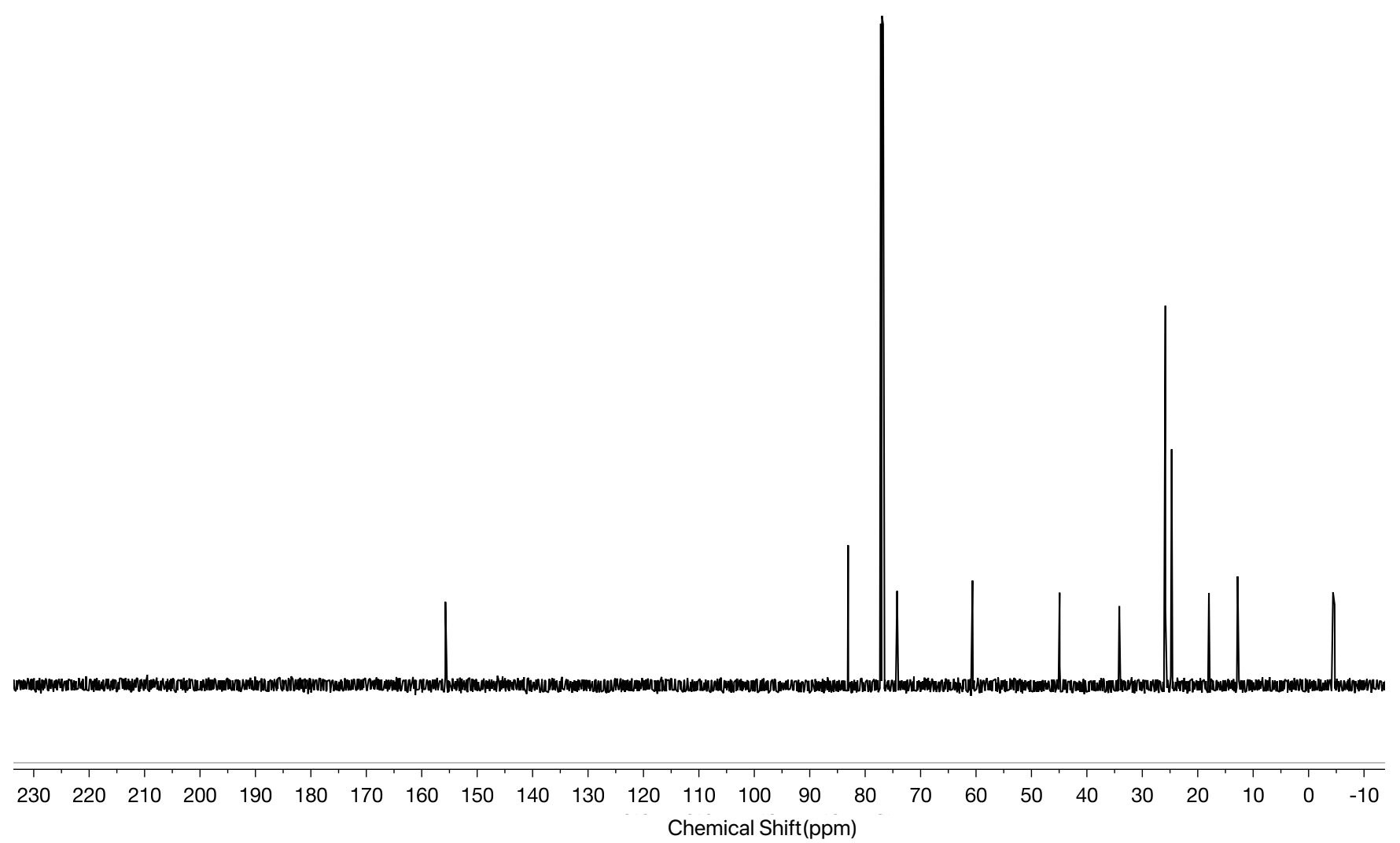
<smiles>CC(C=CB1OC(C)(C)C(C)(C)O1)C([OH2+])CC=O</smiles>
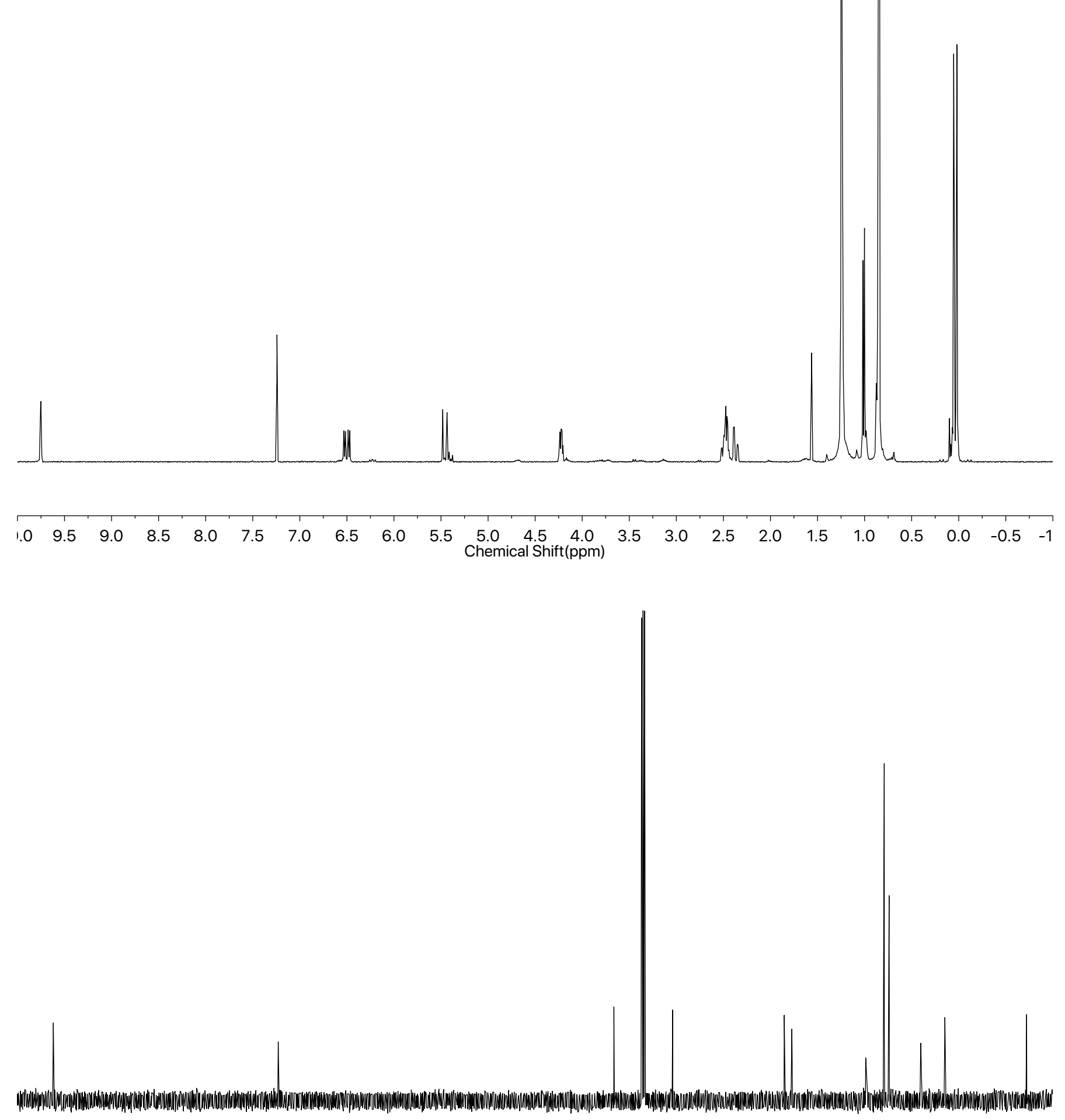

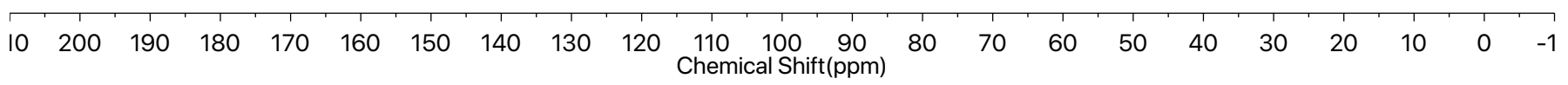


<smiles>CCOP(=O)(CC(=O)N[C@@H](Cc1ccc(OC)c(Cl)c1)C(=O)OC)OCC</smiles>
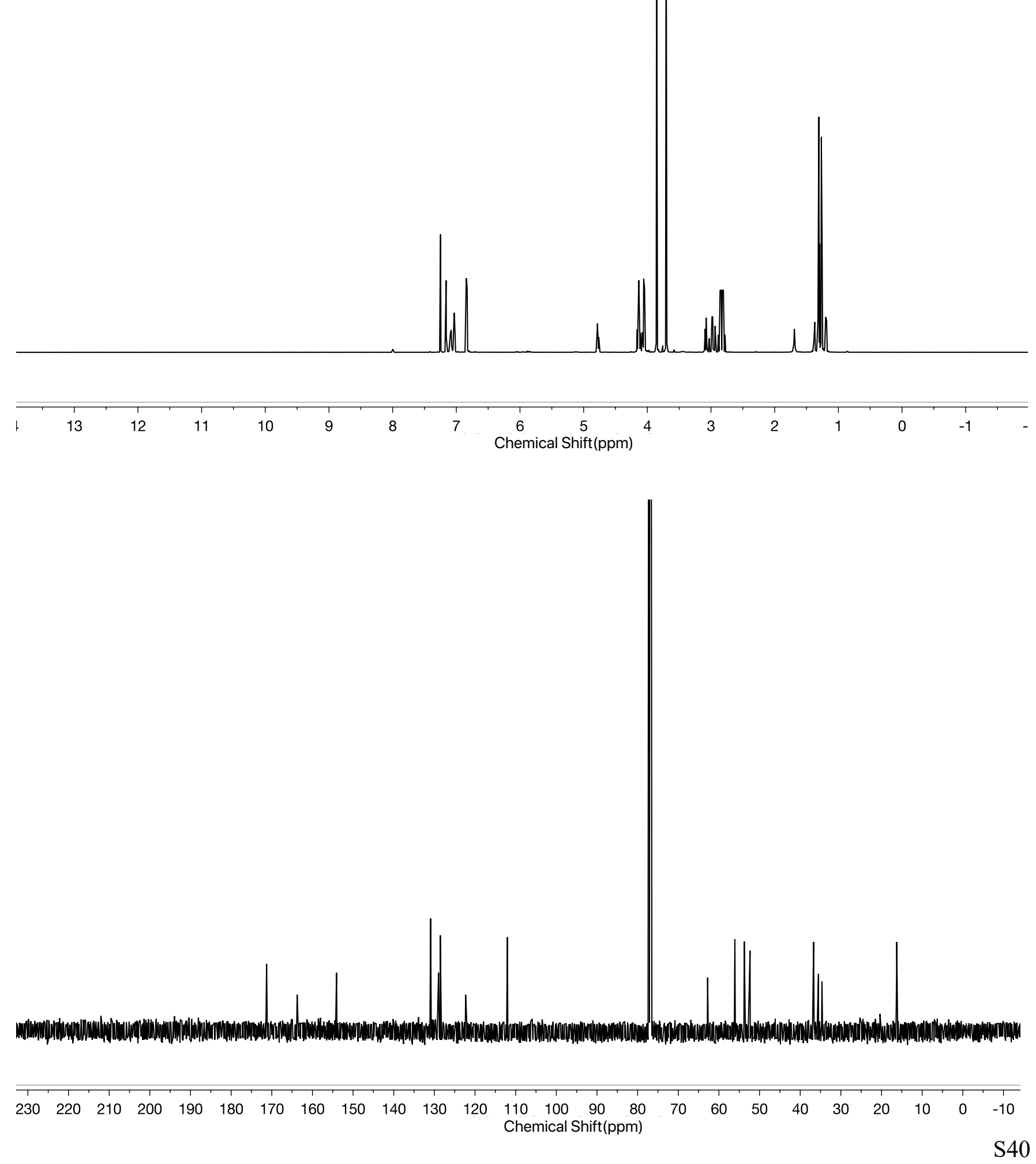

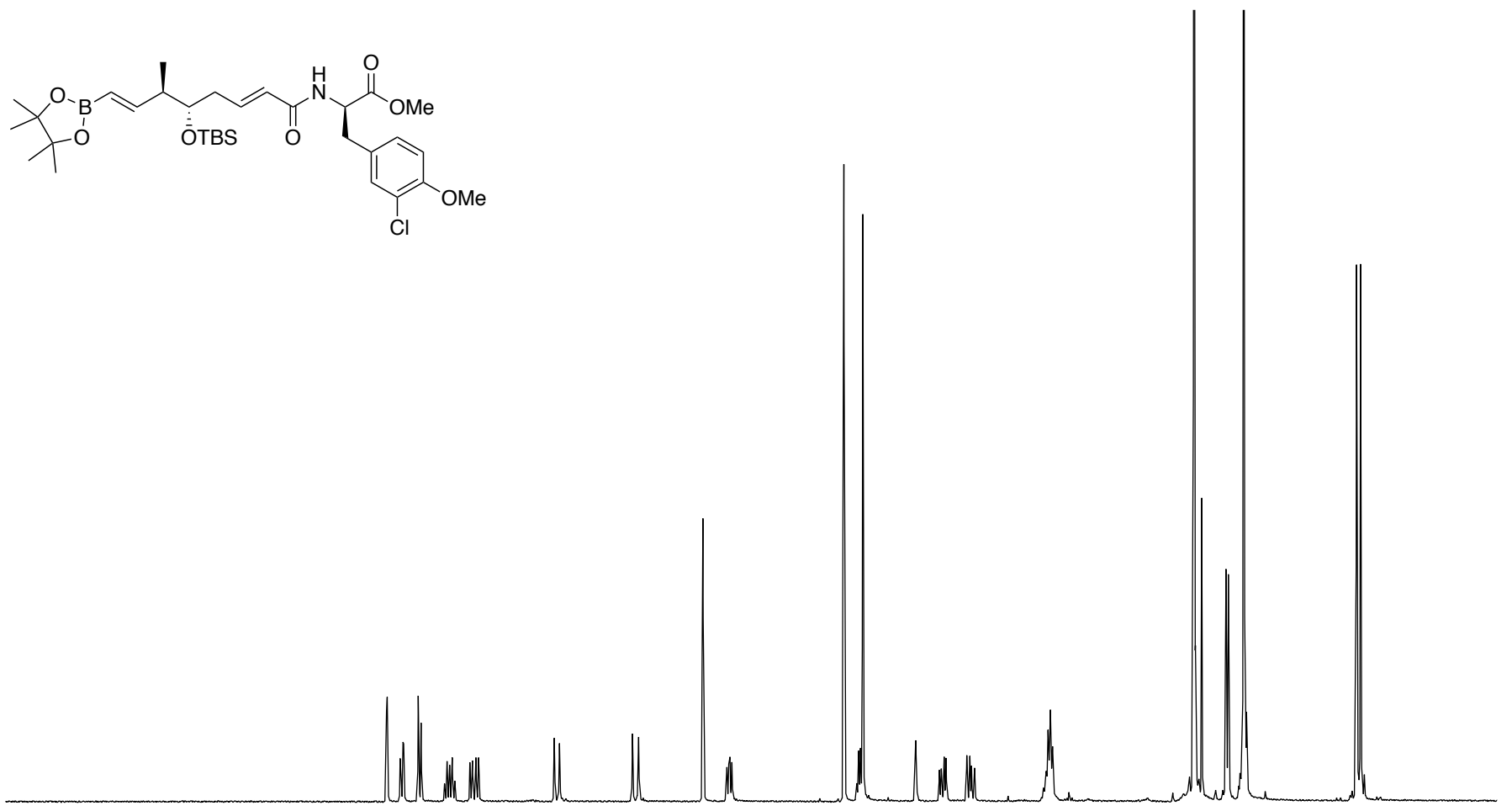

\begin{tabular}{lllllllllllllllllllll}
\hline 1.0 & 9.5 & 9.0 & 8.5 & 8.0 & 7.5 & 7.0 & 6.5 & 6.0 & 5.5 & $\begin{array}{c}5.0 \\
\text { Chemical Shift(ppm) }\end{array}$ & $\begin{array}{c}4.0 \\
\text { Chem }\end{array}$ & 3.0 & 2.5 & 2.0 & 1.5 & 1.0 & 0.5 & 0.0 & -0.5 & -1
\end{tabular}

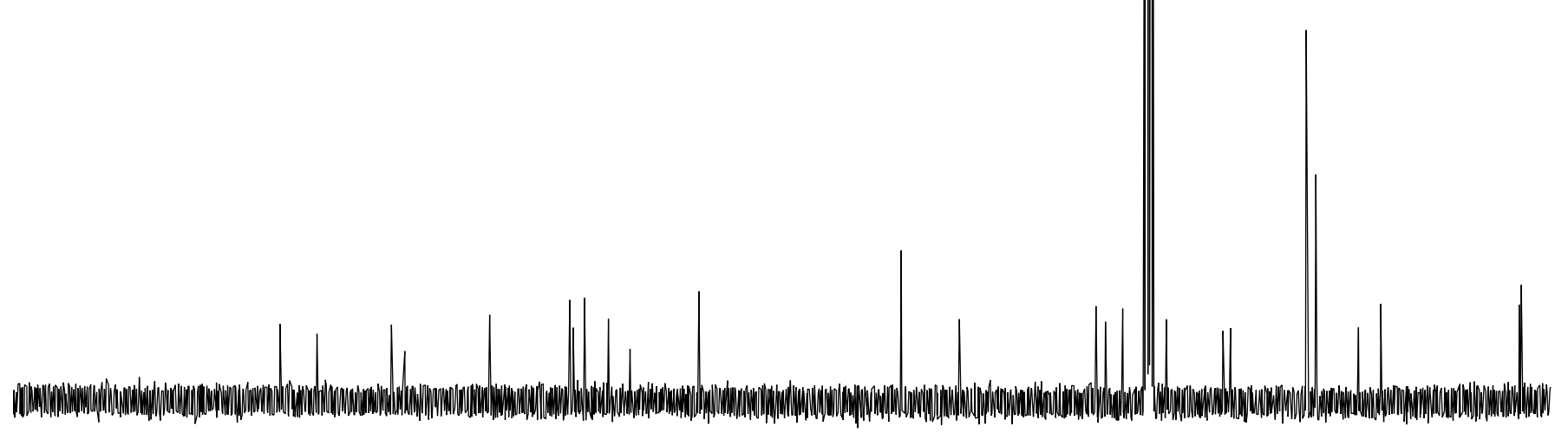

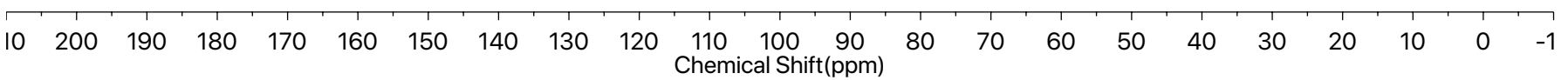



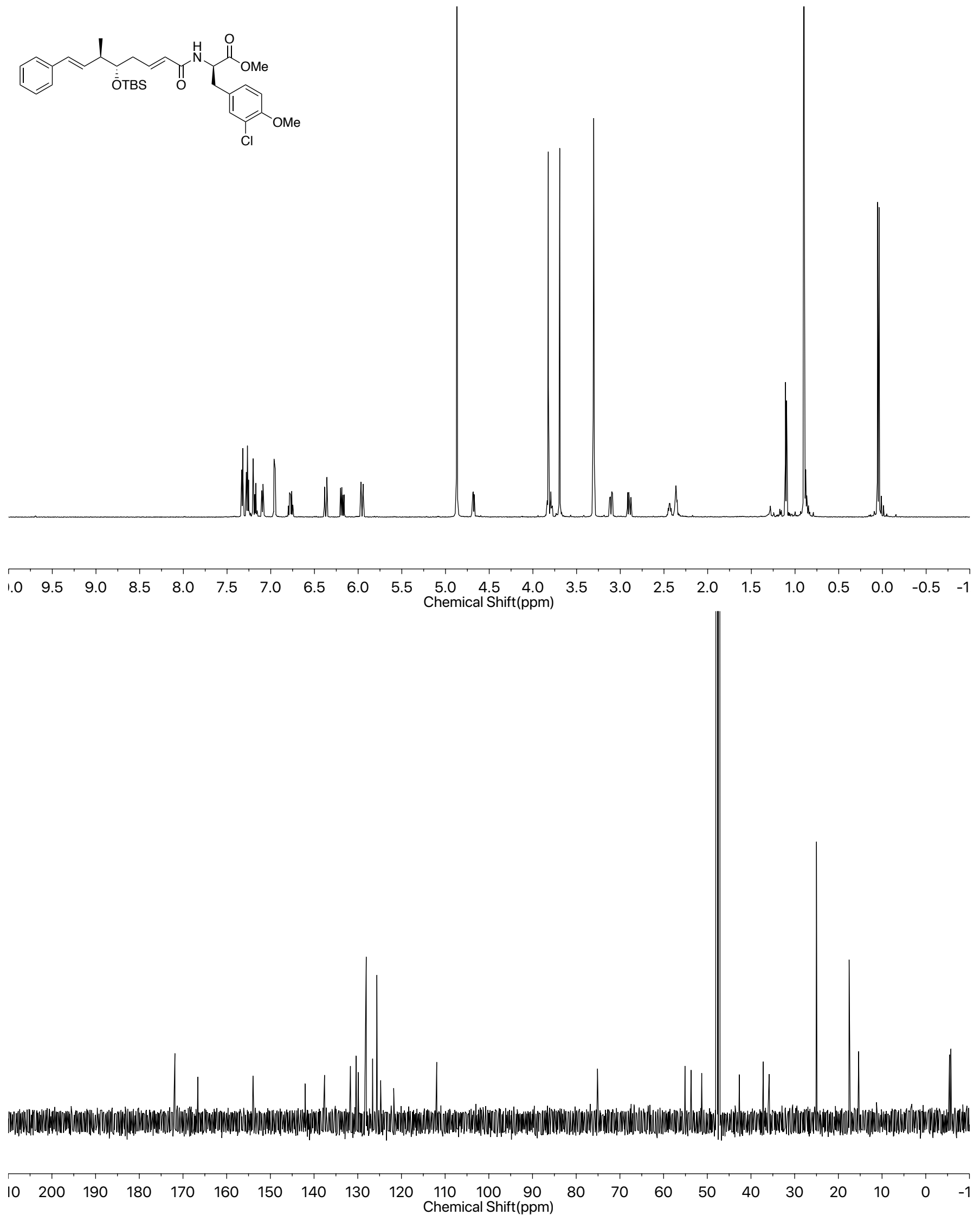

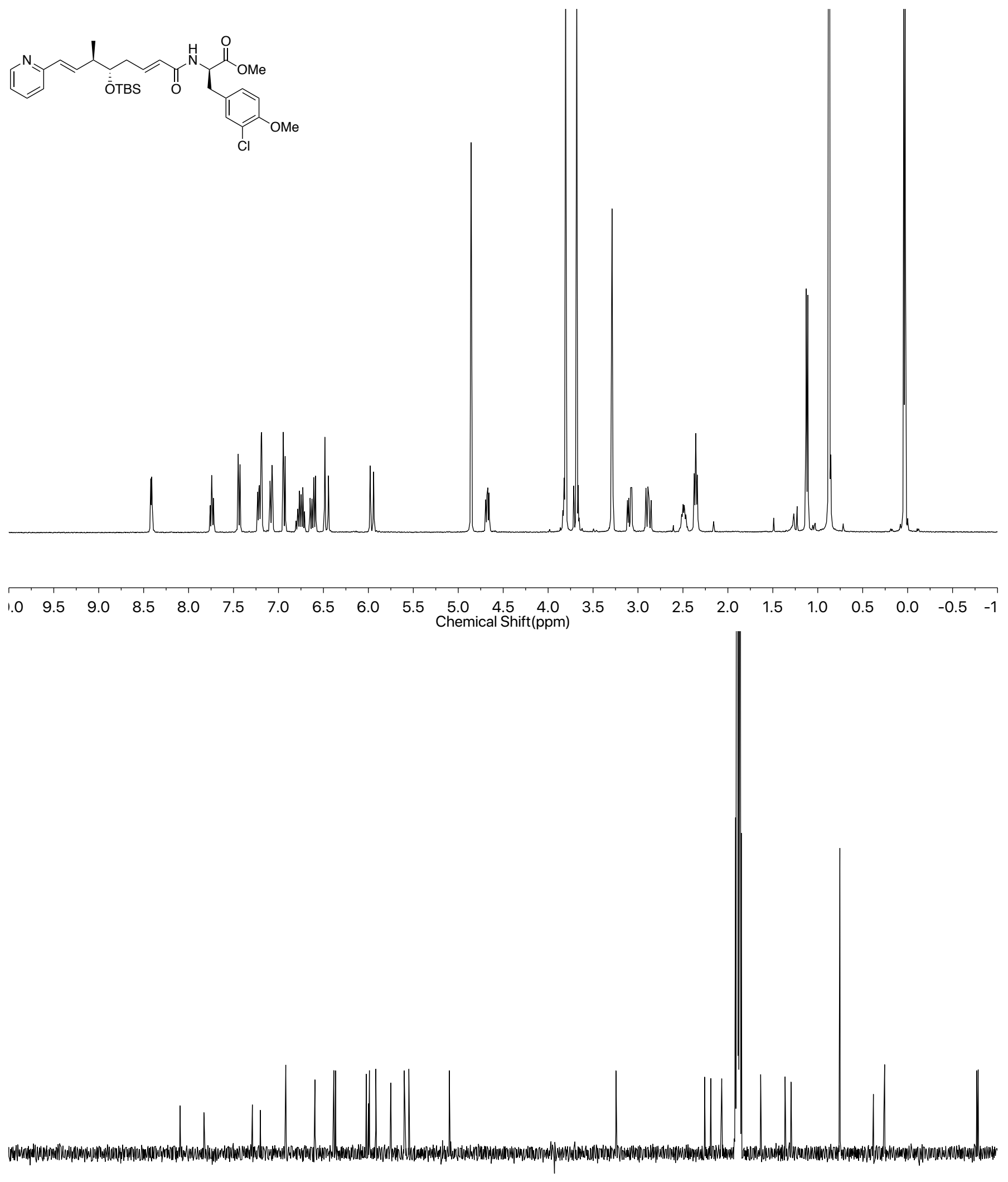

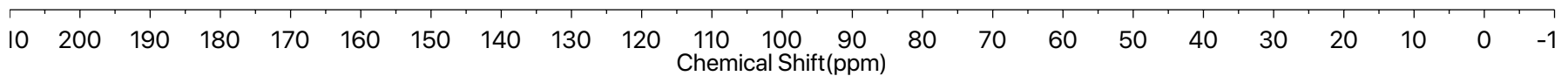



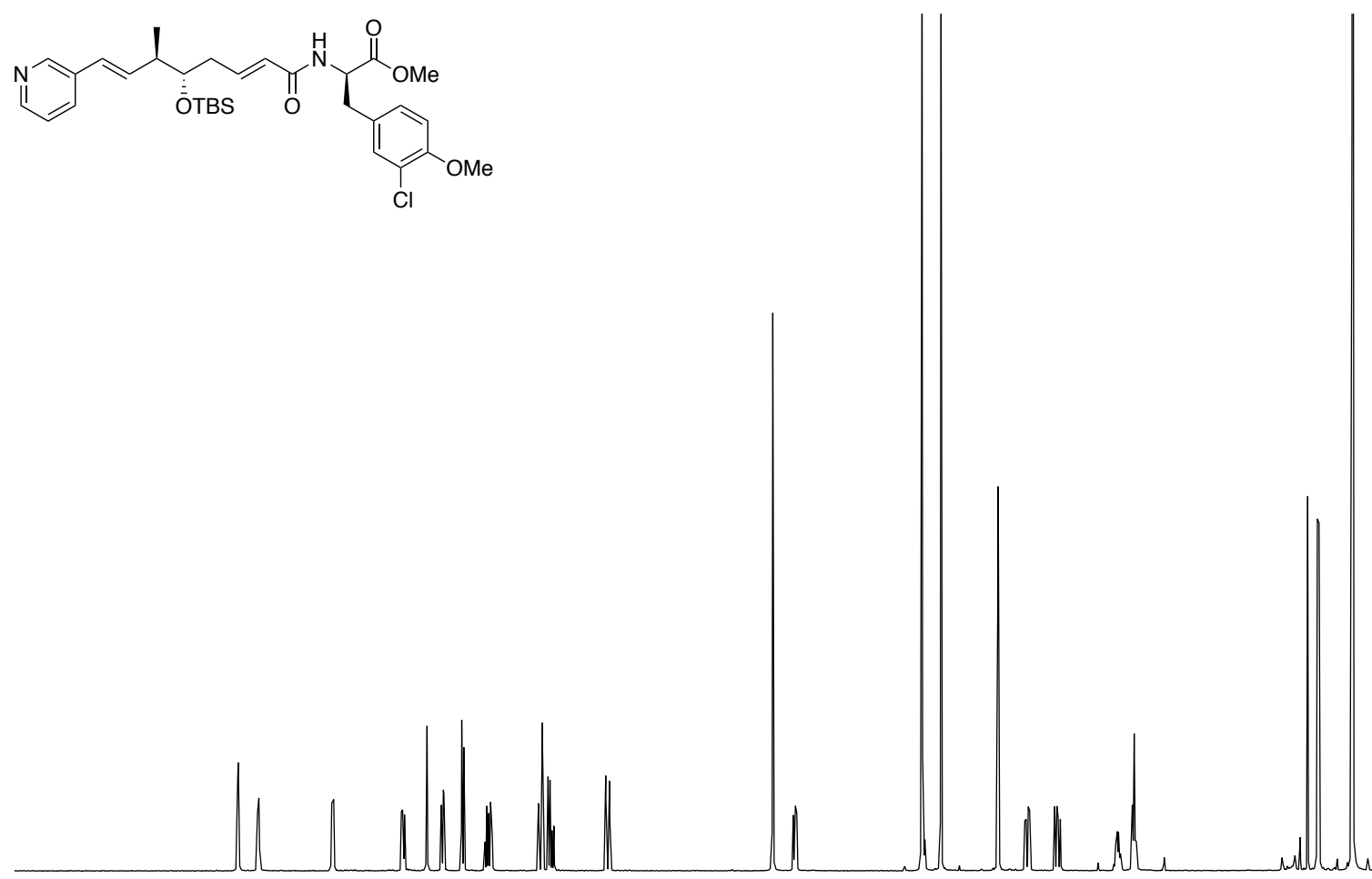

\begin{tabular}{lllllllllllllllllllll}
\hline .0 & 9.5 & 9.0 & 8.5 & 8.0 & 7.5 & 7.0 & 6.5 & 6.0 & 5.5 & $\begin{array}{c}5.0 \\
\text { Chemical Shift(ppm) }\end{array}$ & $\begin{array}{c}4.5 \\
\text { Che }\end{array}$ & 3.0 & 2.5 & 2.0 & 1.5 & 1.0 & 0.5 & 0.0 & -0.5 & -1
\end{tabular}

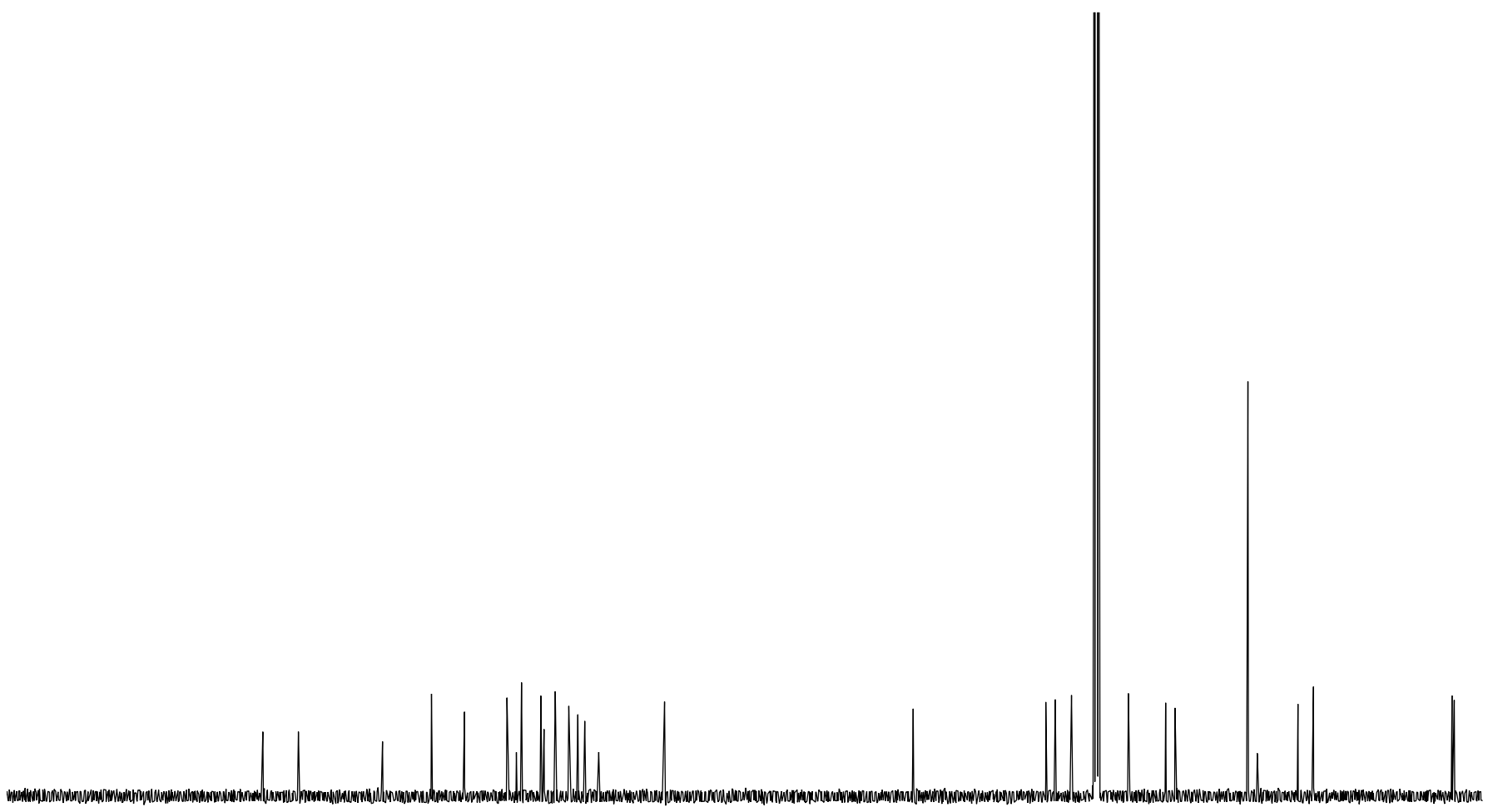

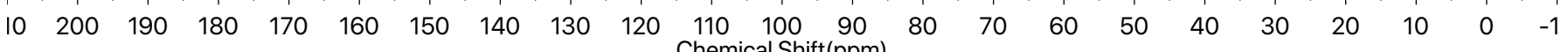



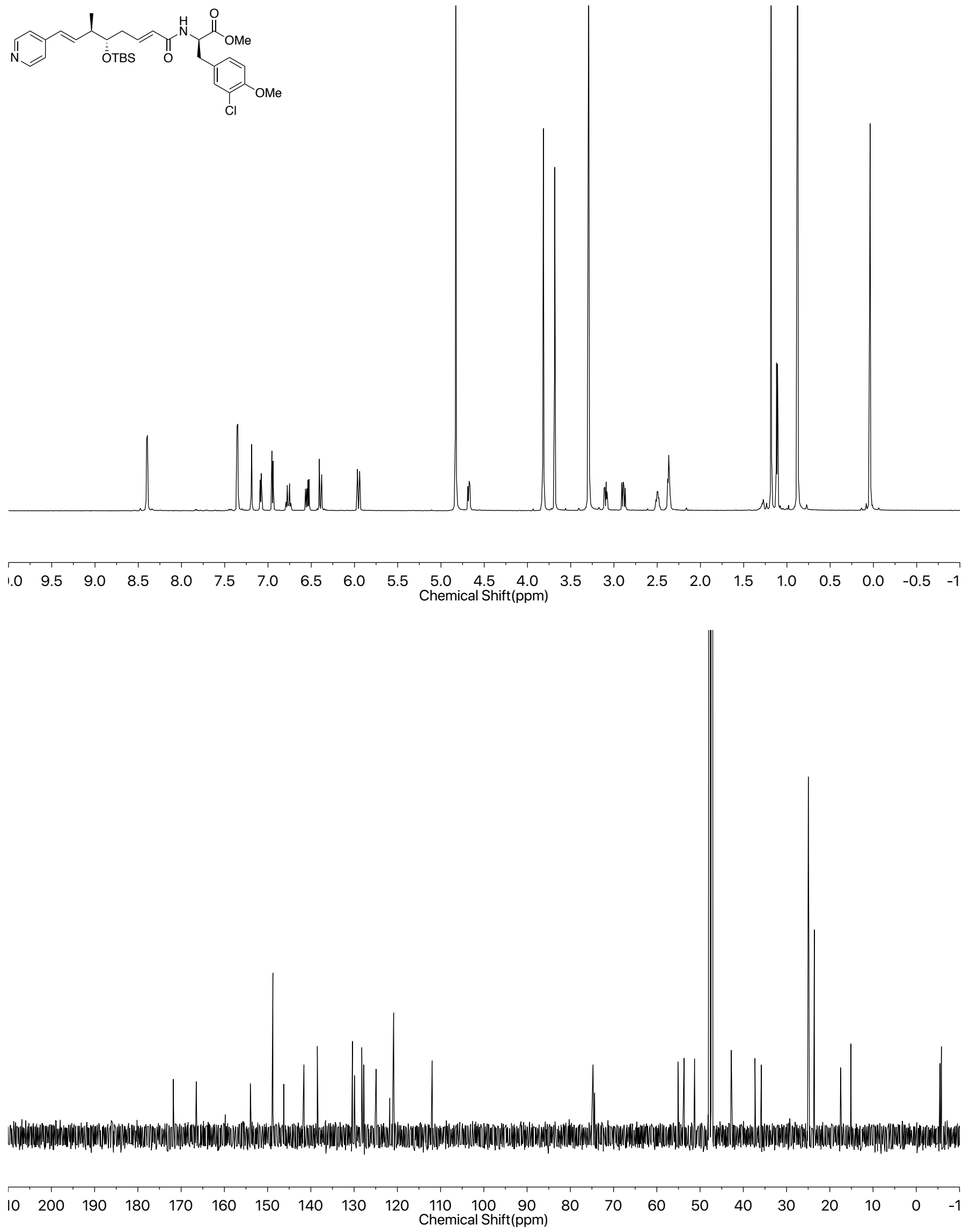

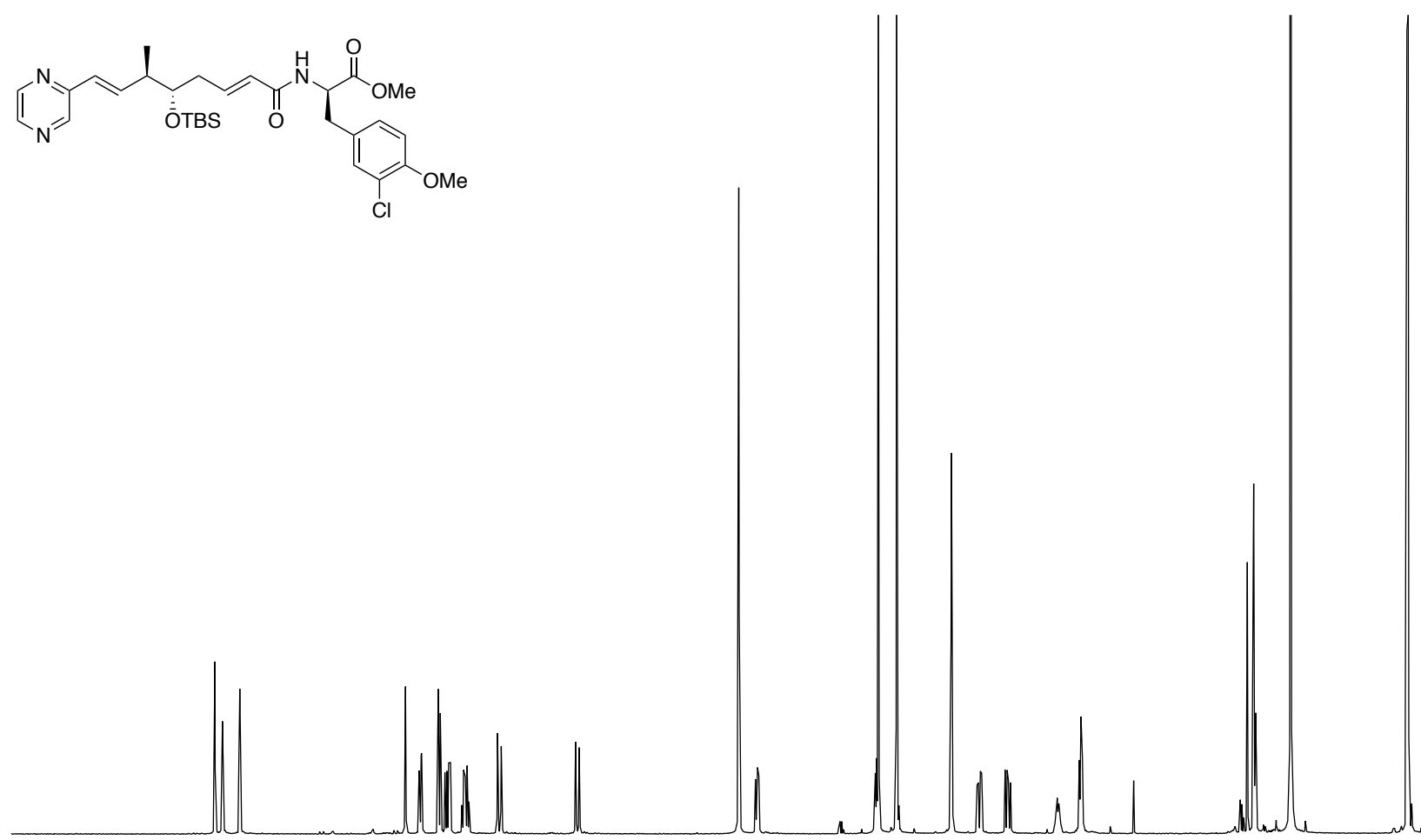

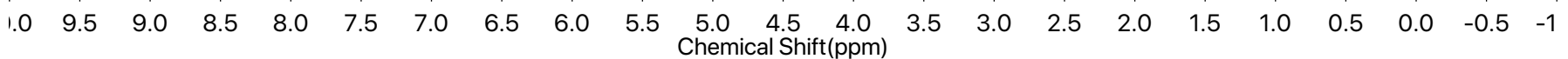
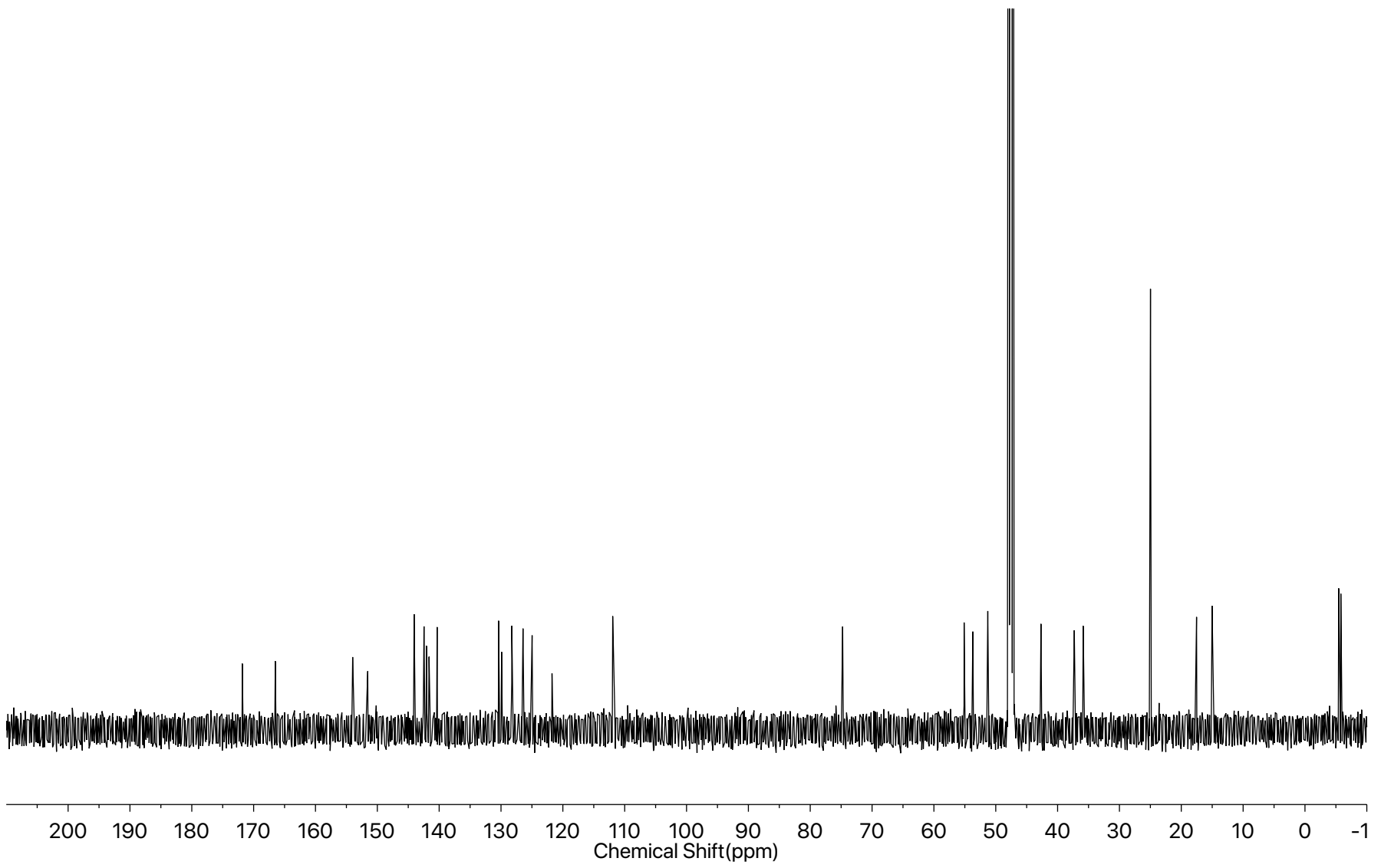

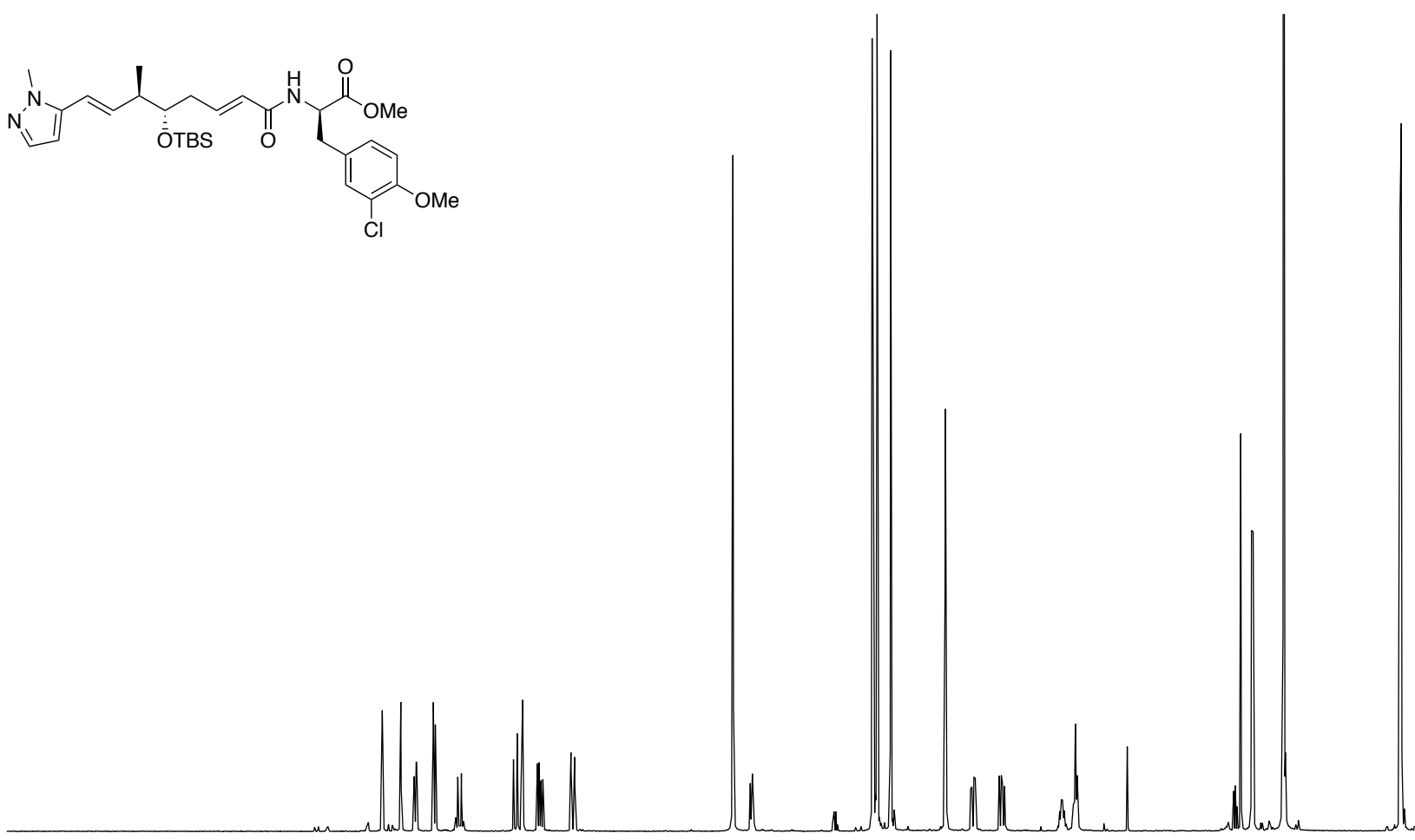

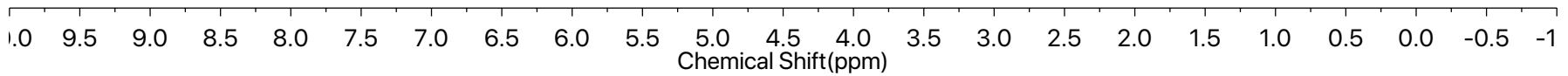

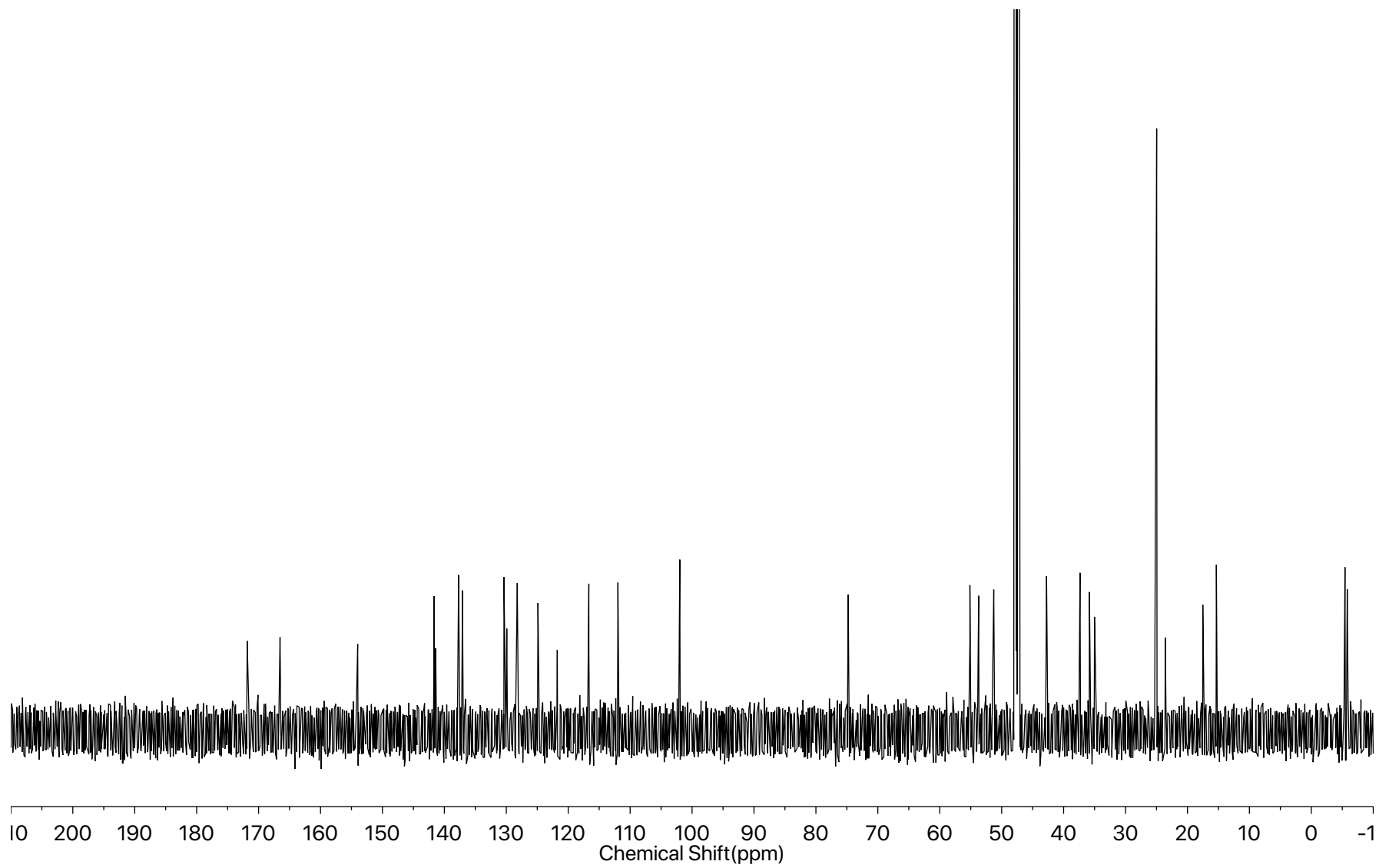




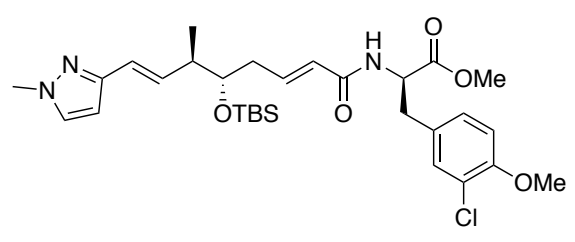

\begin{tabular}{|c|c|c|c|c|c|c|c|c|c|c|c|c|c|c|c|c|c|c|c|c|c|c|c|c|}
\hline 1.0 & 9.5 & 9.0 & 8.5 & 8. & 7. & & 7.0 & 6.5 & 6.0 & 5.5 & $\begin{array}{c}5.0 \\
\text { Chemi }\end{array}$ & $\begin{array}{r}4.5 \\
\text { cal Sh }\end{array}$ & $\begin{array}{c}4.0 \\
t(p p m)\end{array}$ & 3.5 & 3.0 & & & 2.0 & 1.5 & & .0 & 0.5 & -0.5 & -1 \\
\hline
\end{tabular}

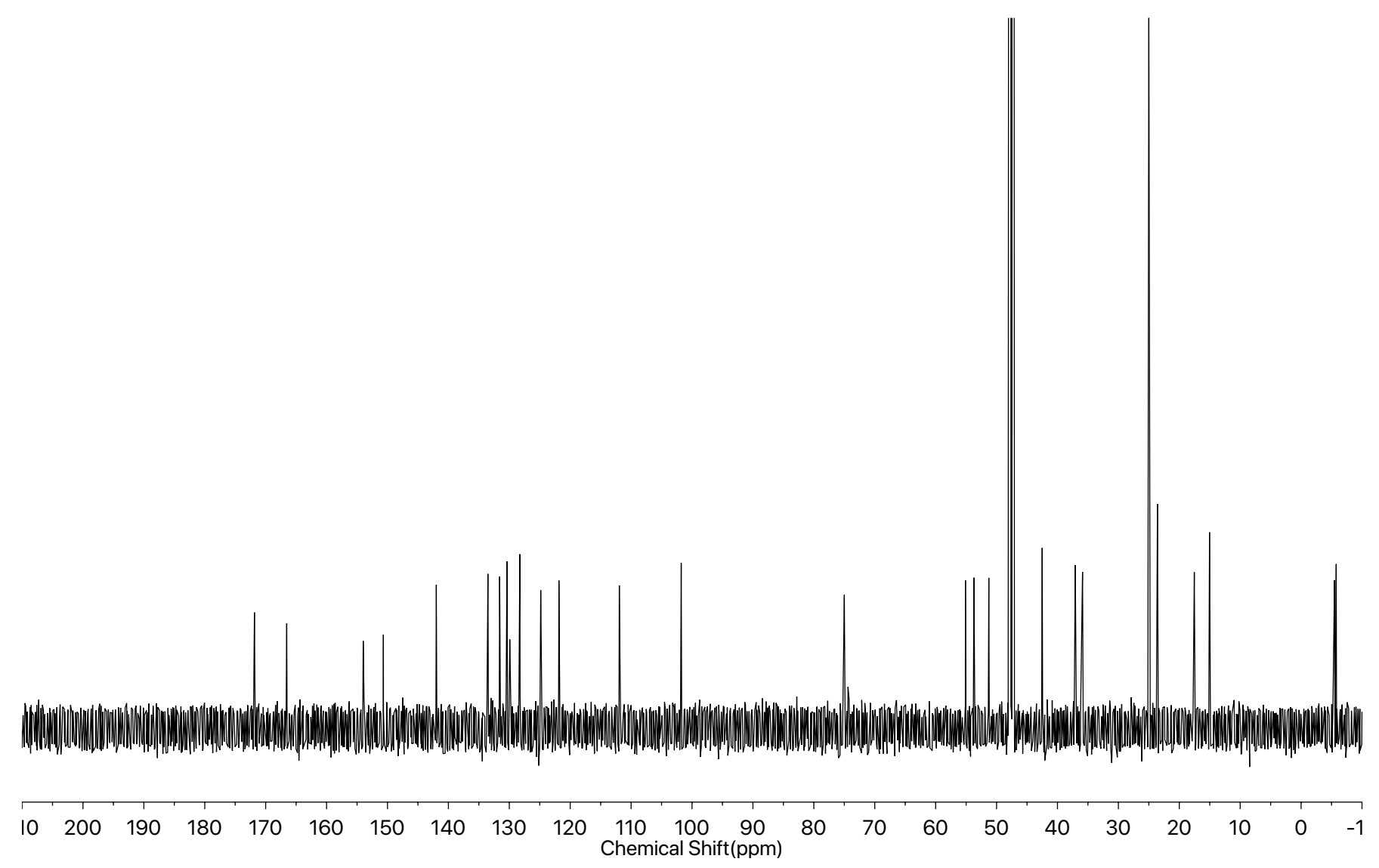



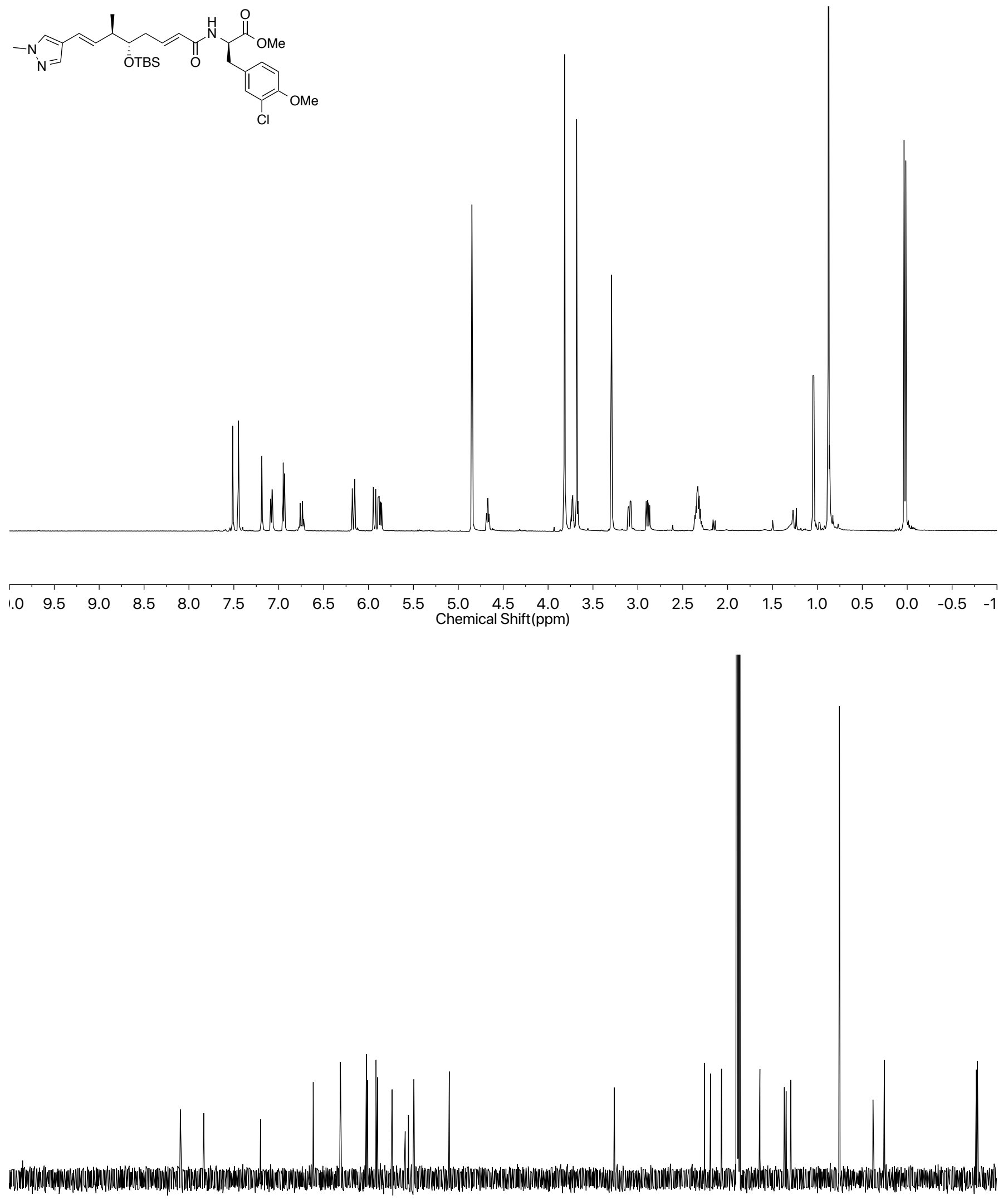

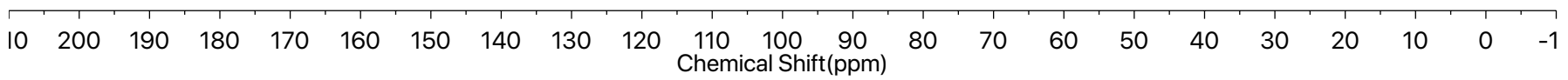



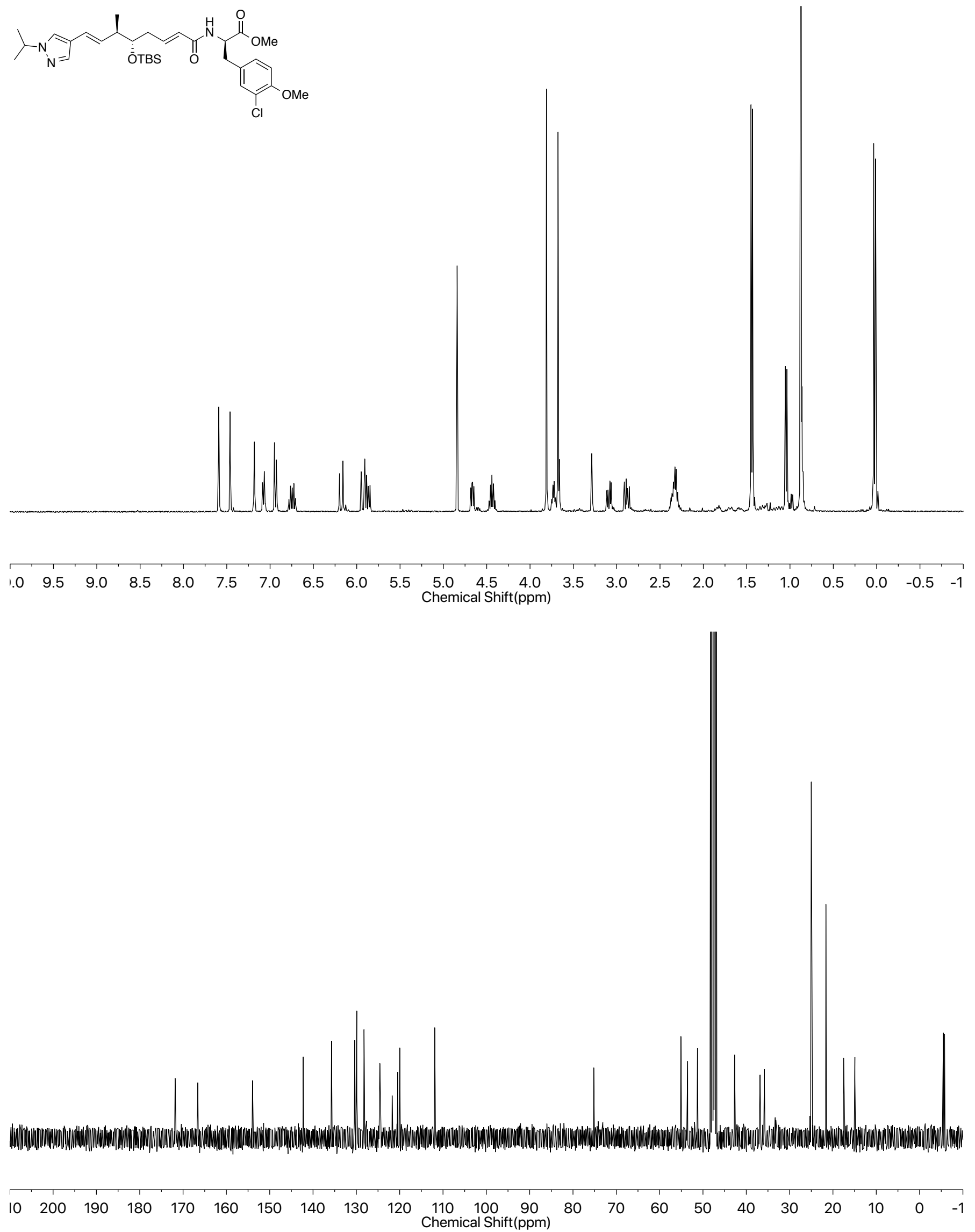

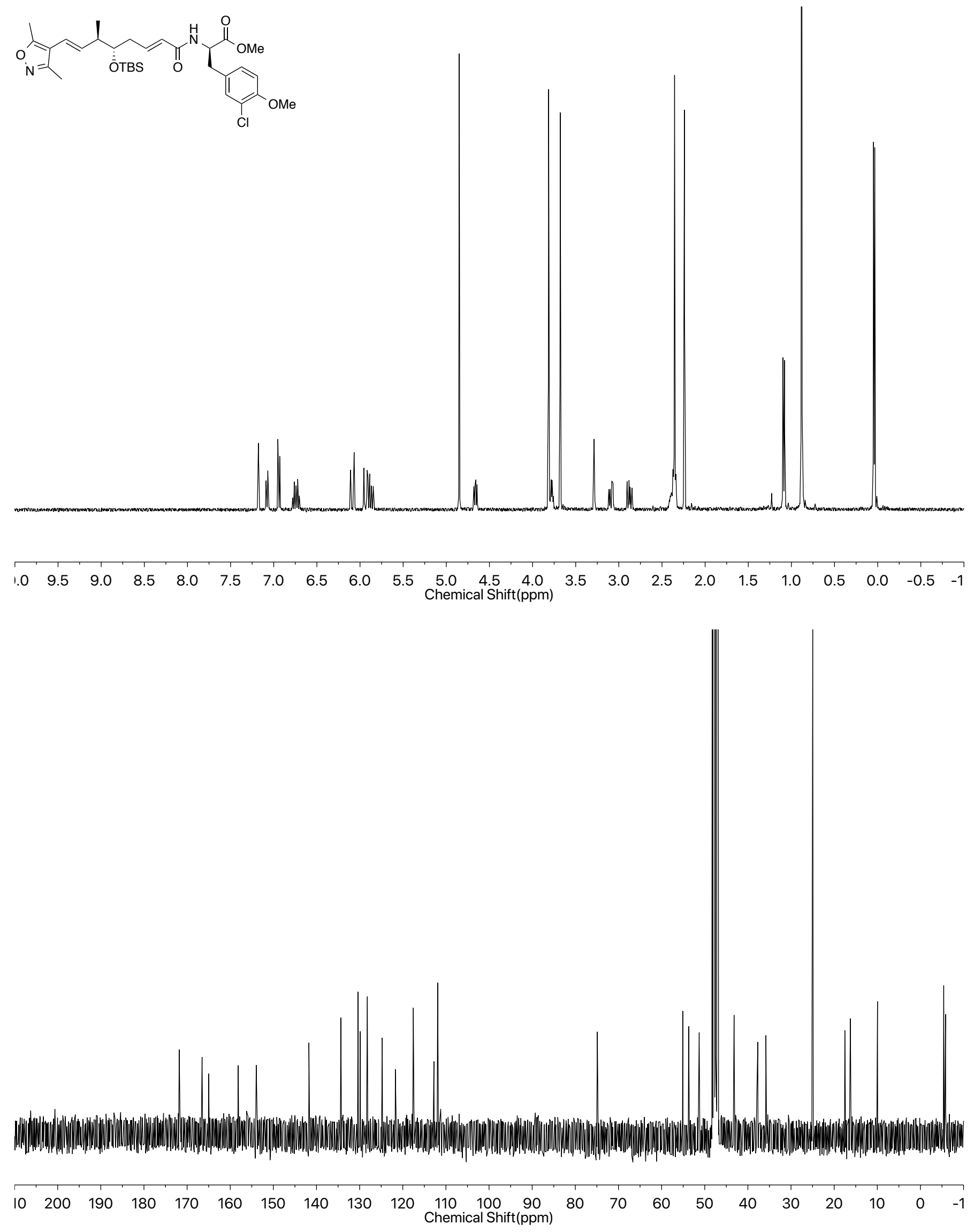


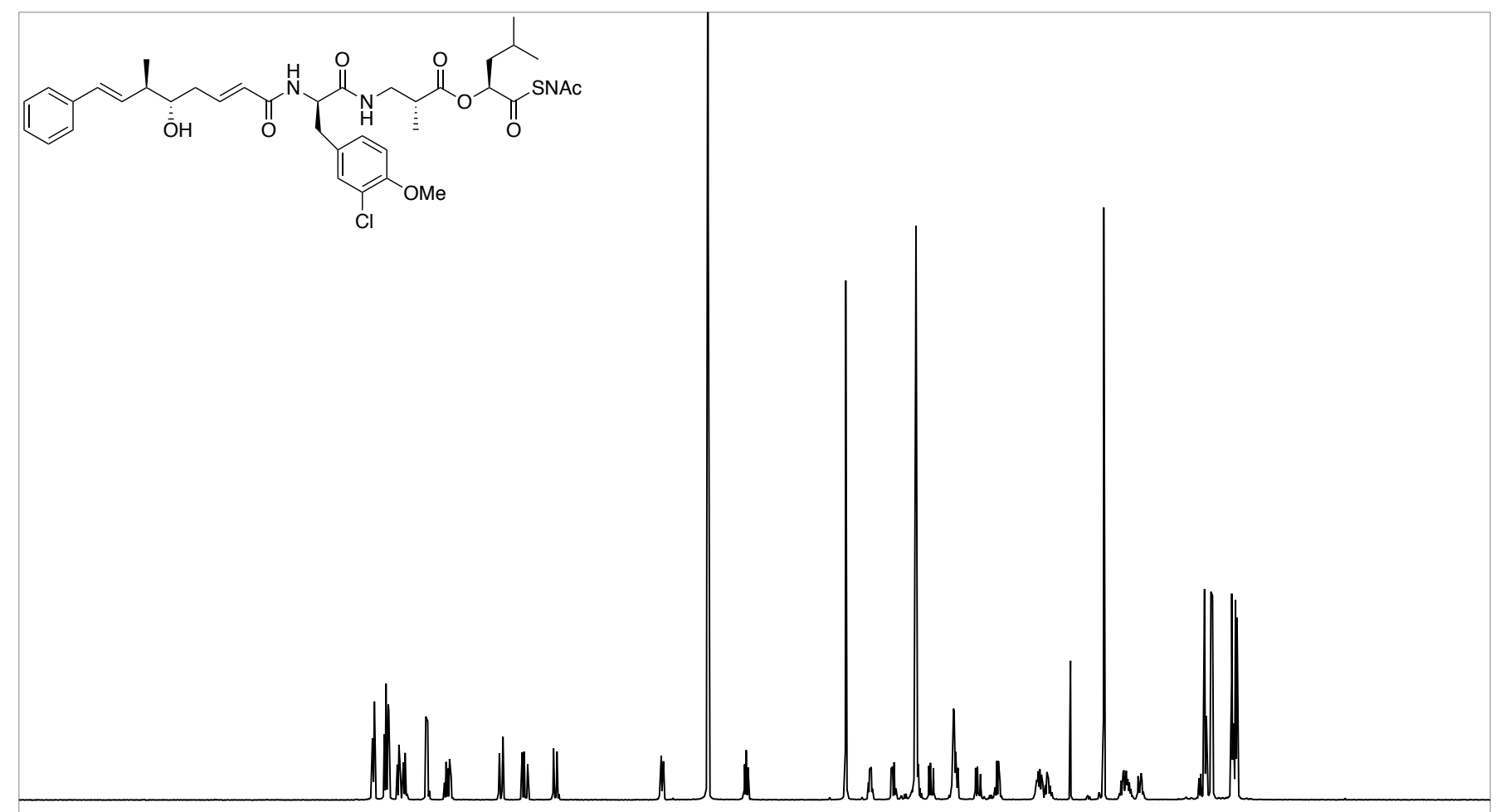

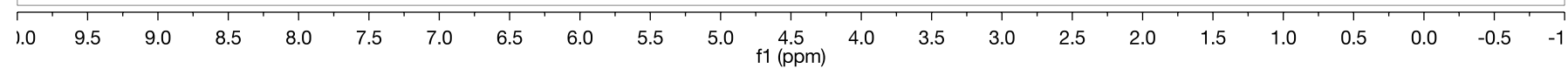

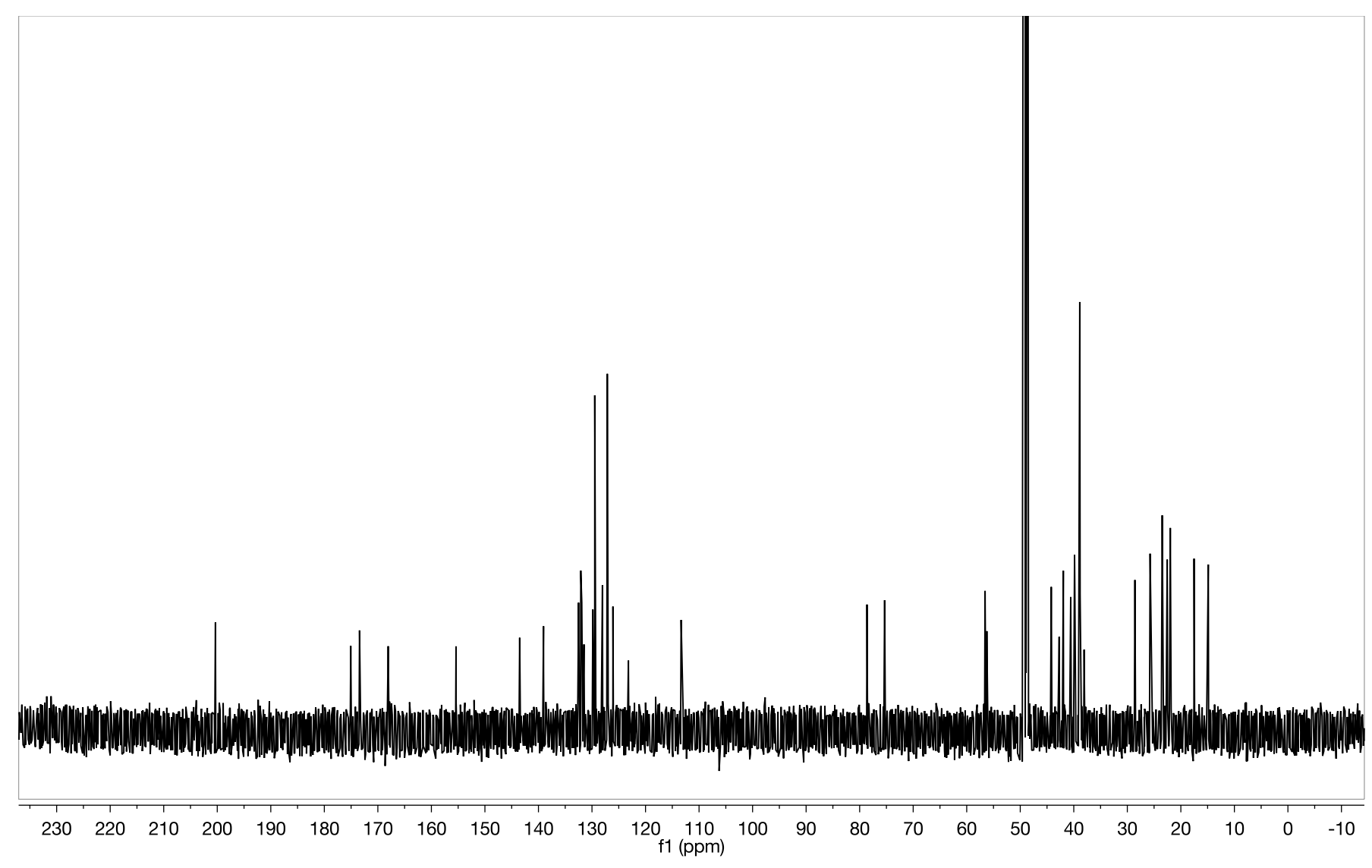




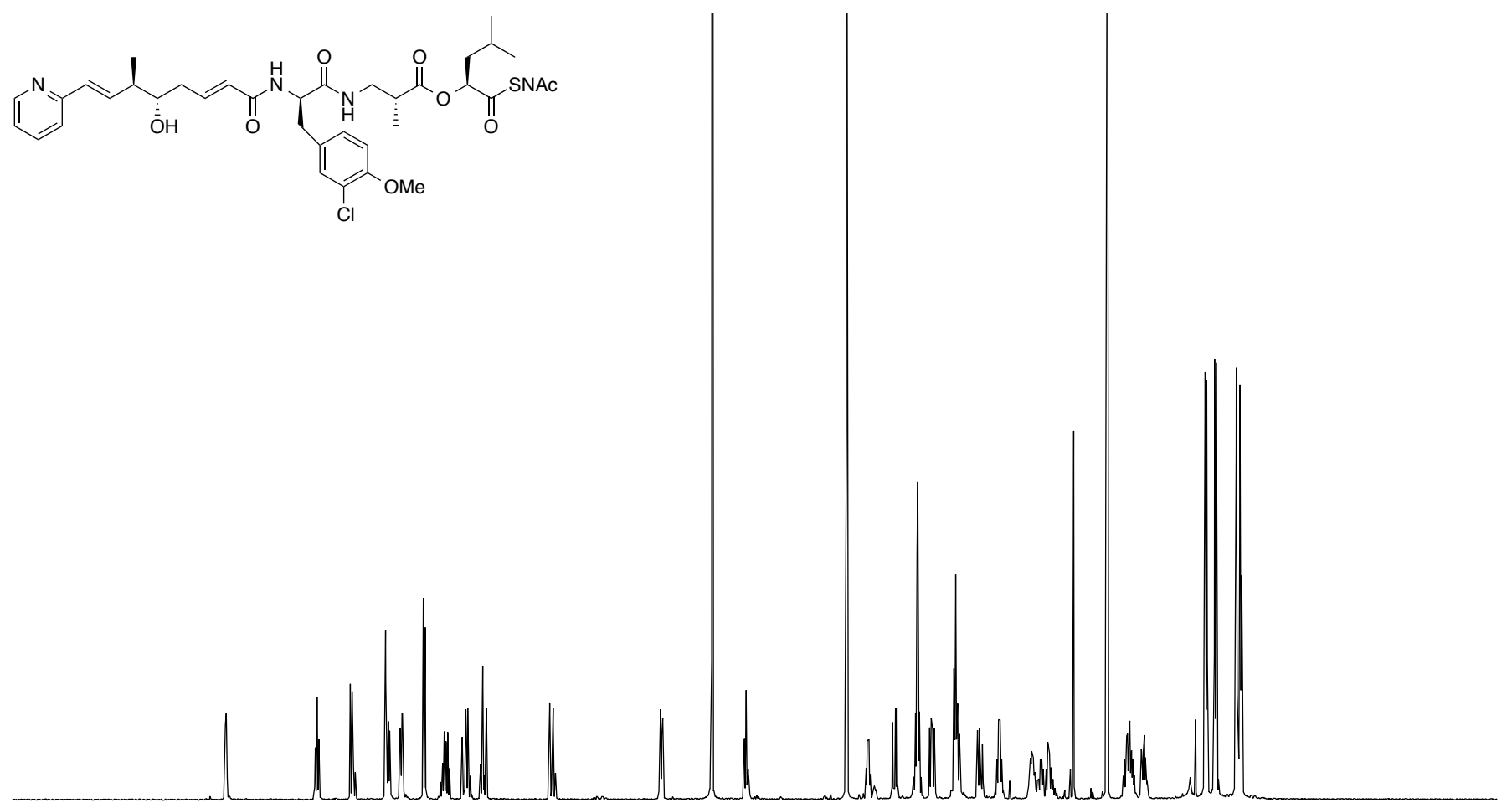

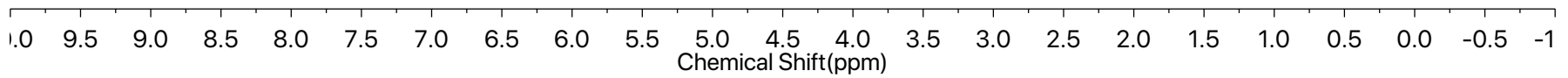

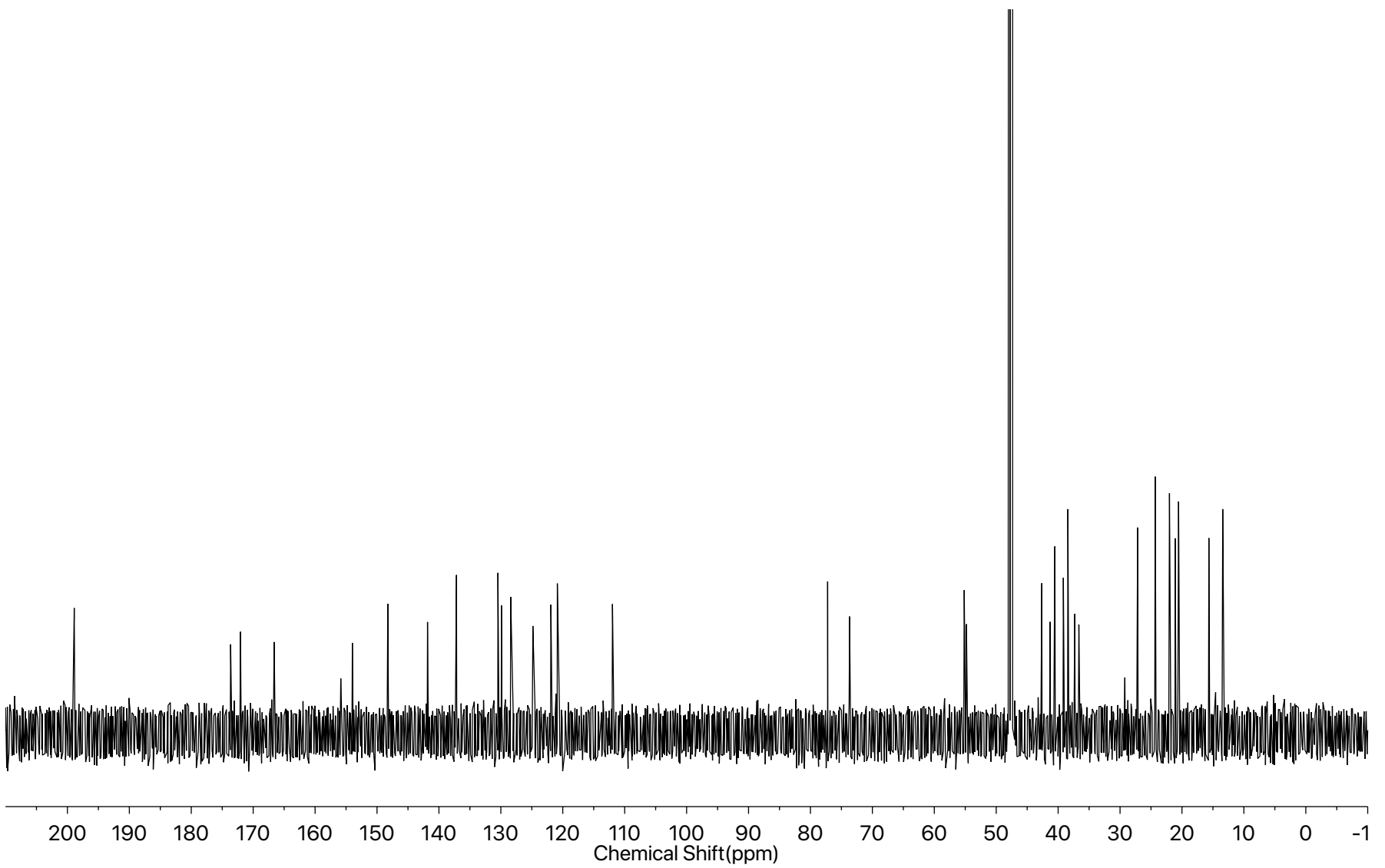




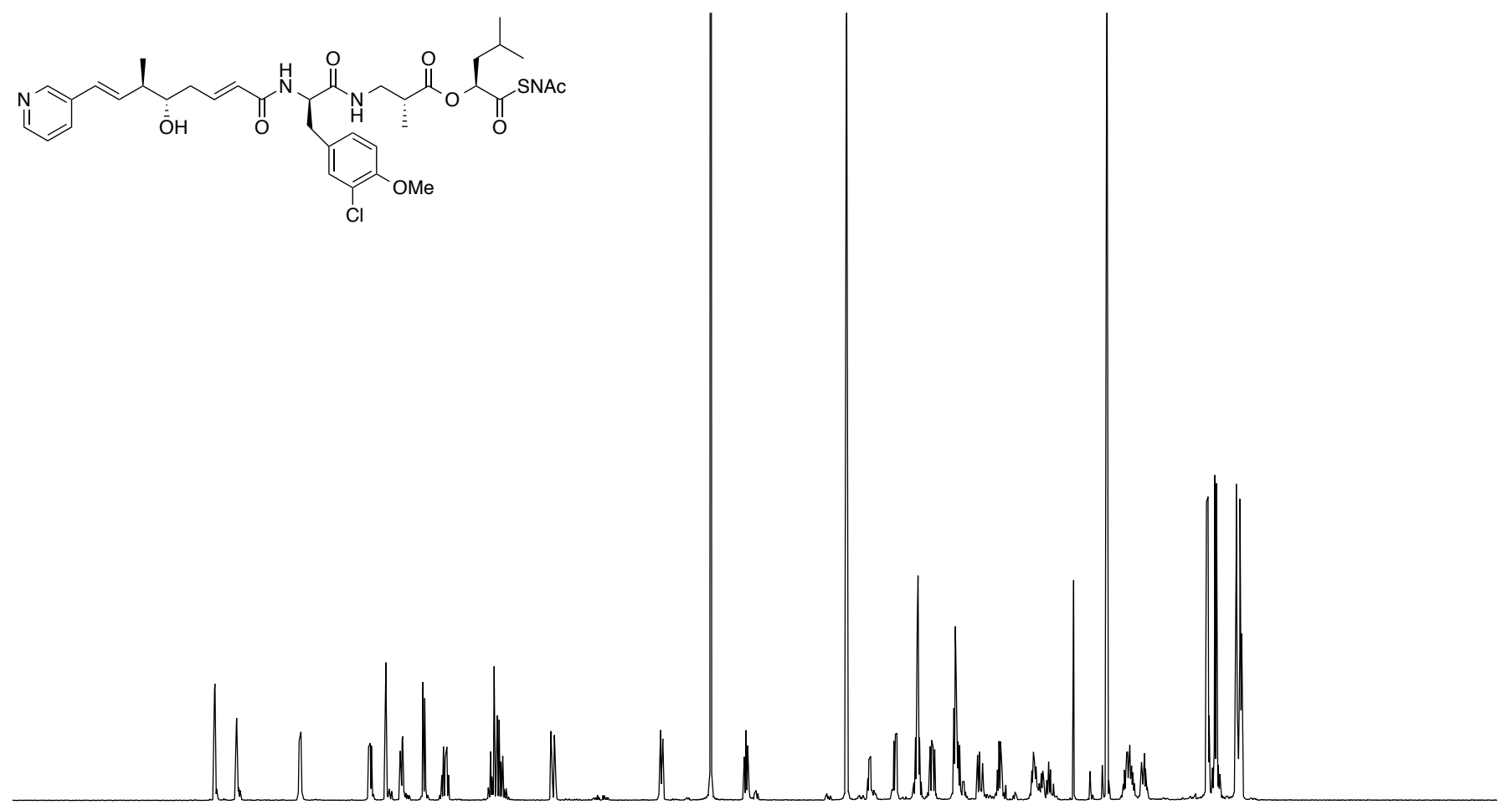

\begin{tabular}{lllllllllllllllllllll}
\hline .0 & 9.5 & 9.0 & 8.5 & 8.0 & 7.5 & 7.0 & 6.5 & 6.0 & 5.5 & $\begin{array}{c}5.0 \\
\text { Chemical Shift(ppm) }\end{array}$ & 4.5 & 3.0 & 2.5 & 2.0 & 1.5 & 1.0 & 0.5 & 0.0 & -0.5 & -1
\end{tabular}

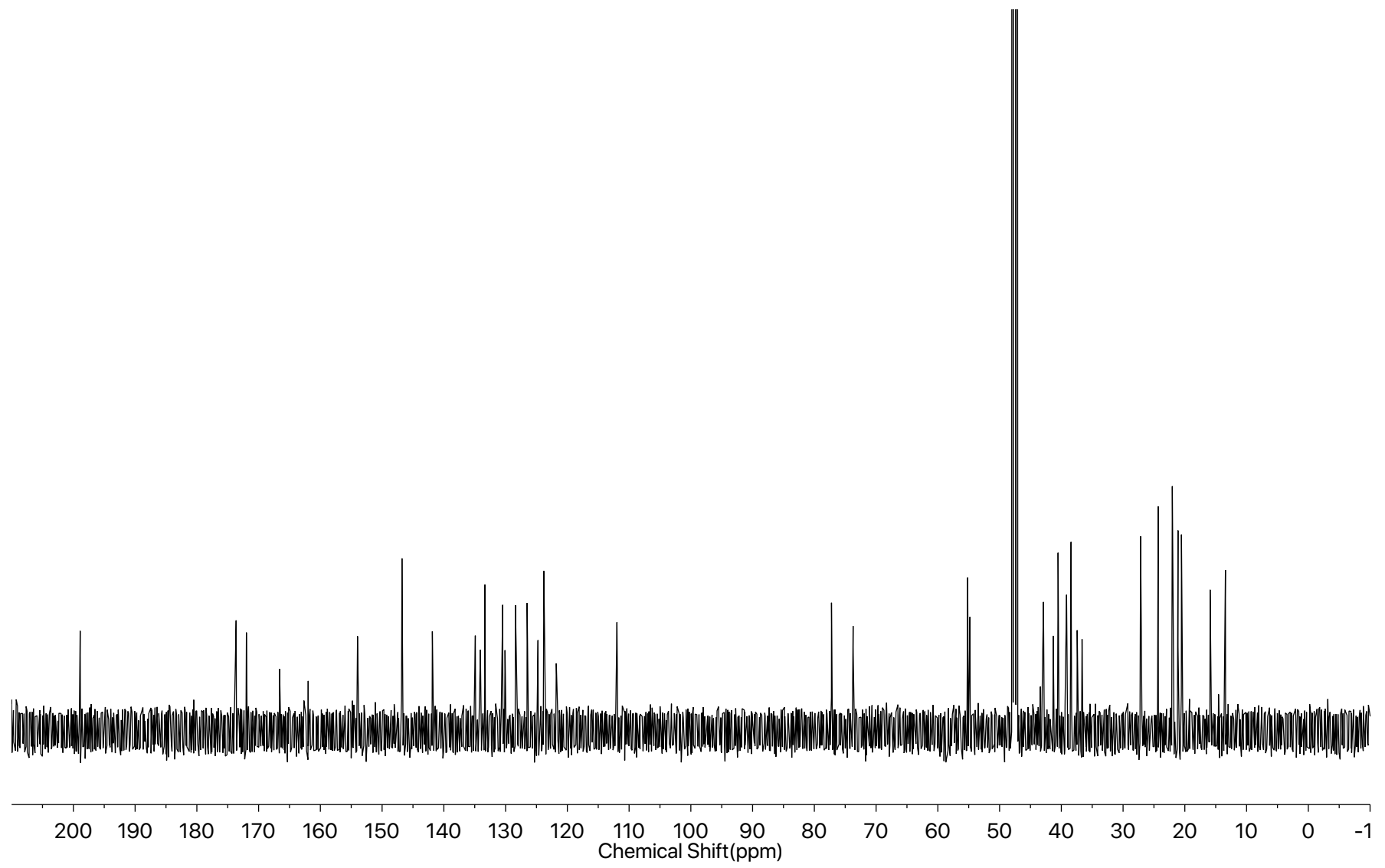



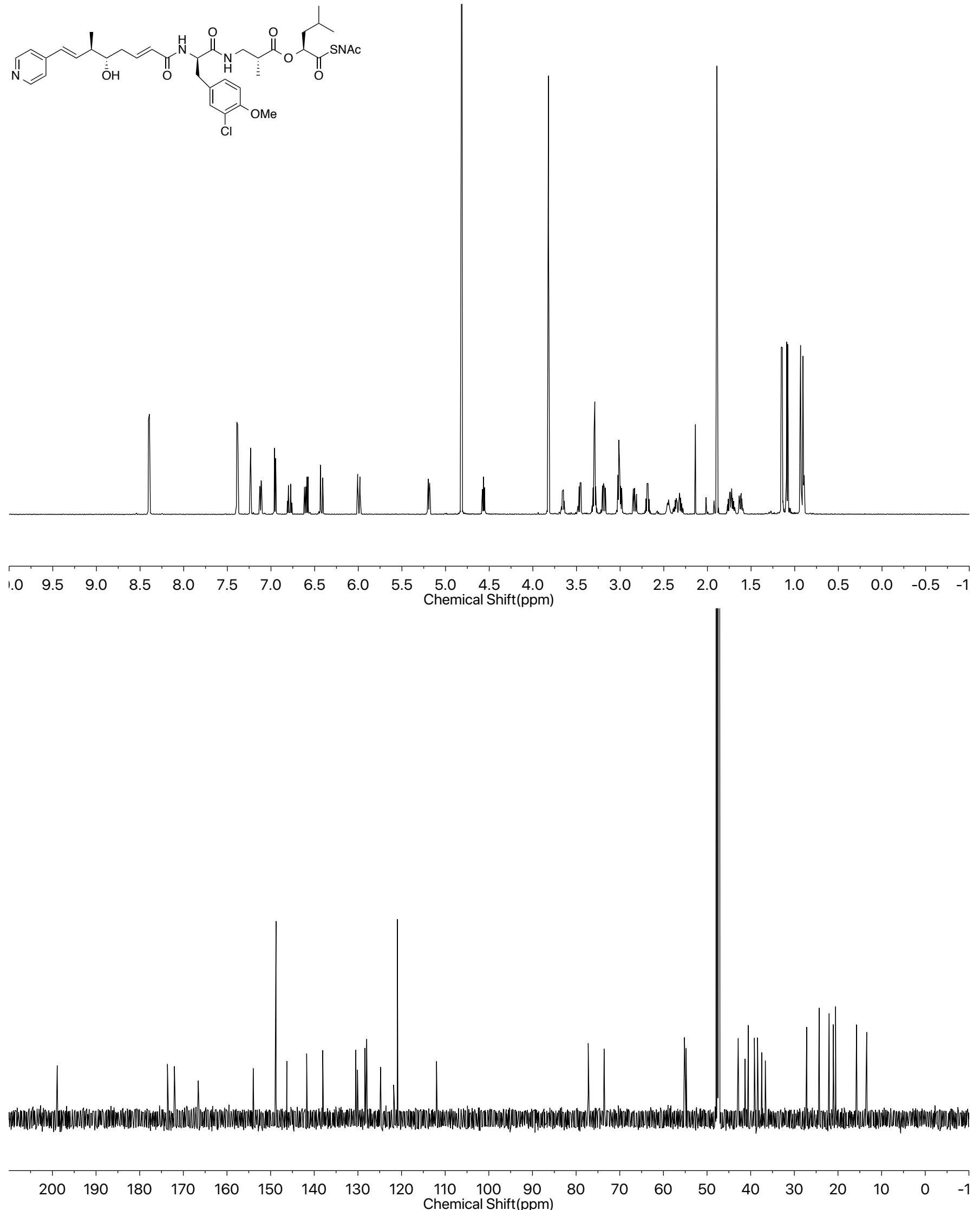

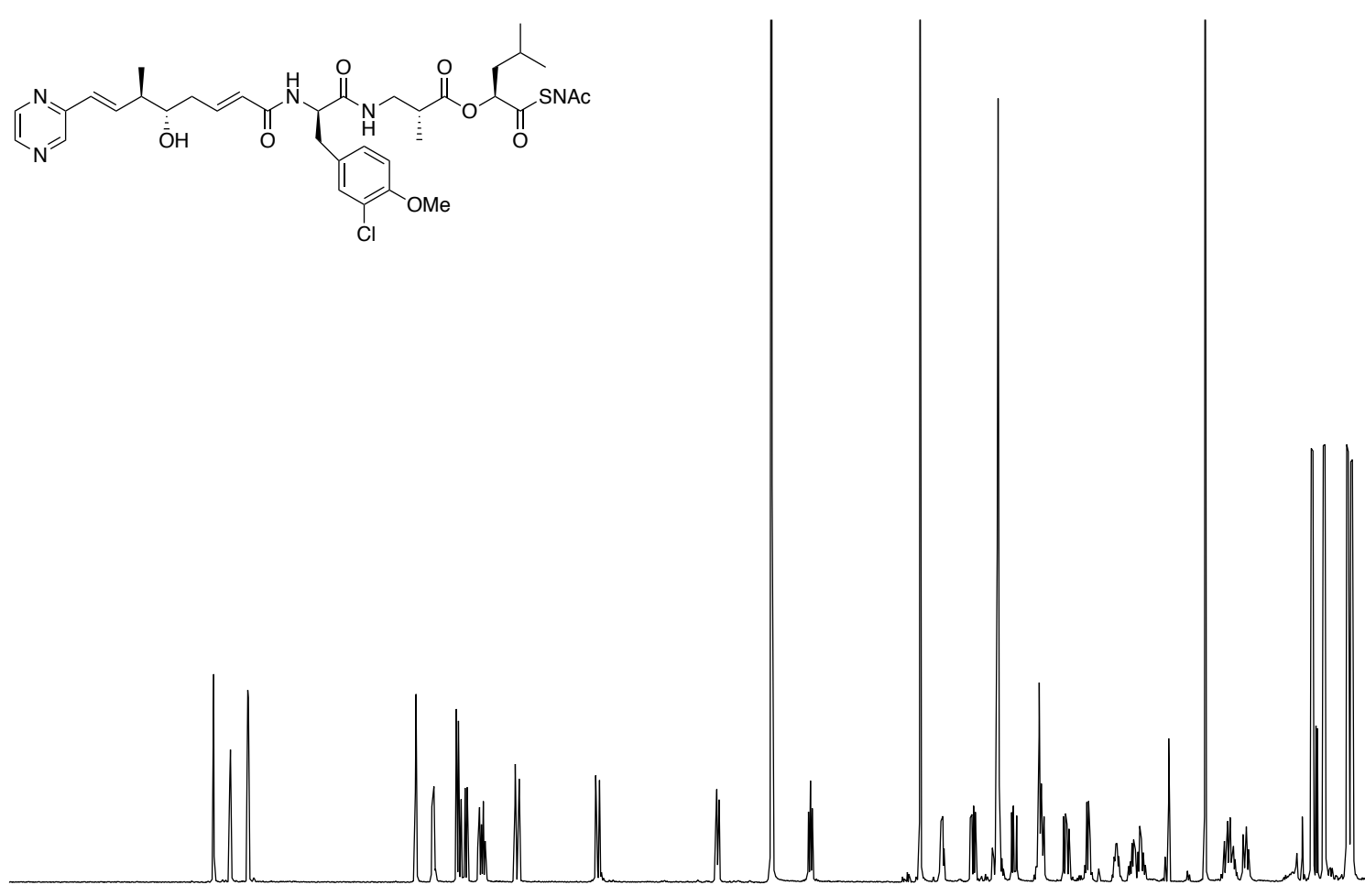

\begin{tabular}{lllllllllllllllllllll}
\hline .0 & 9.5 & 9.0 & 8.5 & 8.0 & 7.5 & 7.0 & 6.5 & 6.0 & 5.5 & $\begin{array}{c}5.0 \\
\text { Chemical Shift(ppm) }\end{array}$ & $\begin{array}{llll}4.5 \\
\text { (ppm }\end{array}$ & 3.0 & 2.5 & 2.0 & 1.5 & 1.0 & 0.5 & 0.0 & -0.5 & -1
\end{tabular}

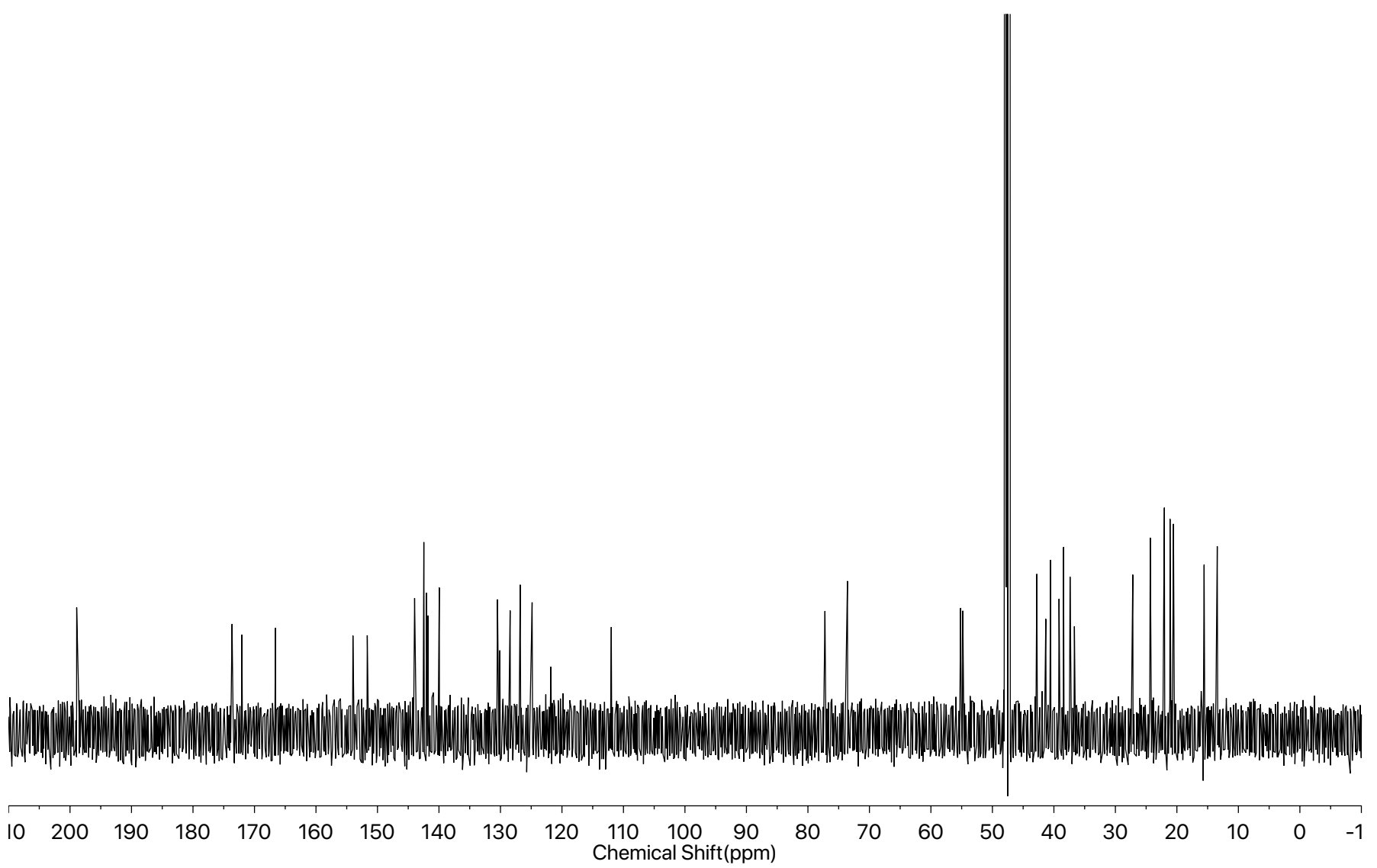



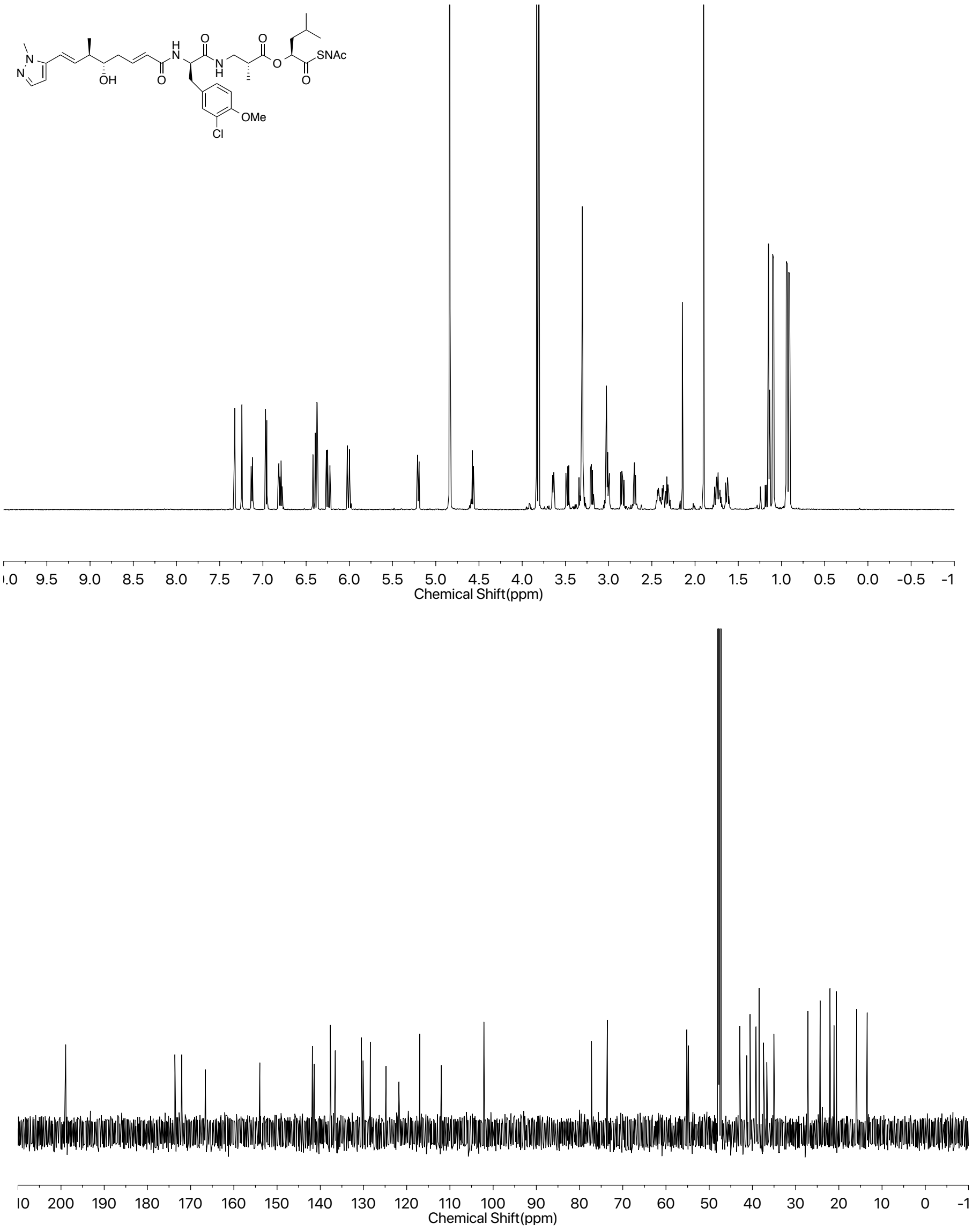


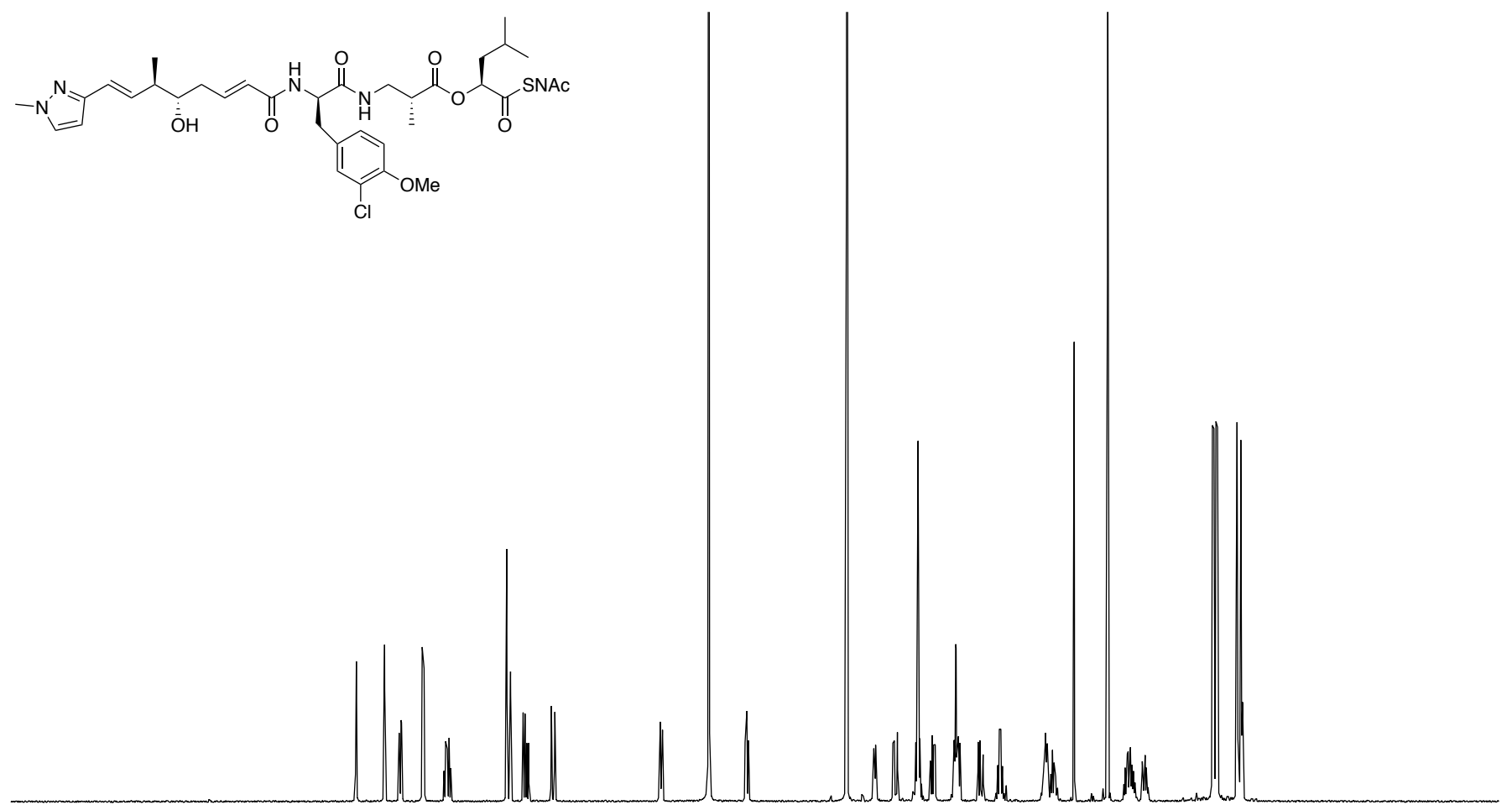

\begin{tabular}{lllllllllllllllllllll}
\hline .0 & 9.5 & 9.0 & 8.5 & 8.0 & 7.5 & 7.0 & 6.5 & 6.0 & 5.5 & $\begin{array}{c}5.0 \\
\text { Chemical Shift(ppm) }\end{array}$ & 4.5 & 3.0 & 2.5 & 2.0 & 1.5 & 1.0 & 0.5 & 0.0 & -0.5 & -1
\end{tabular}

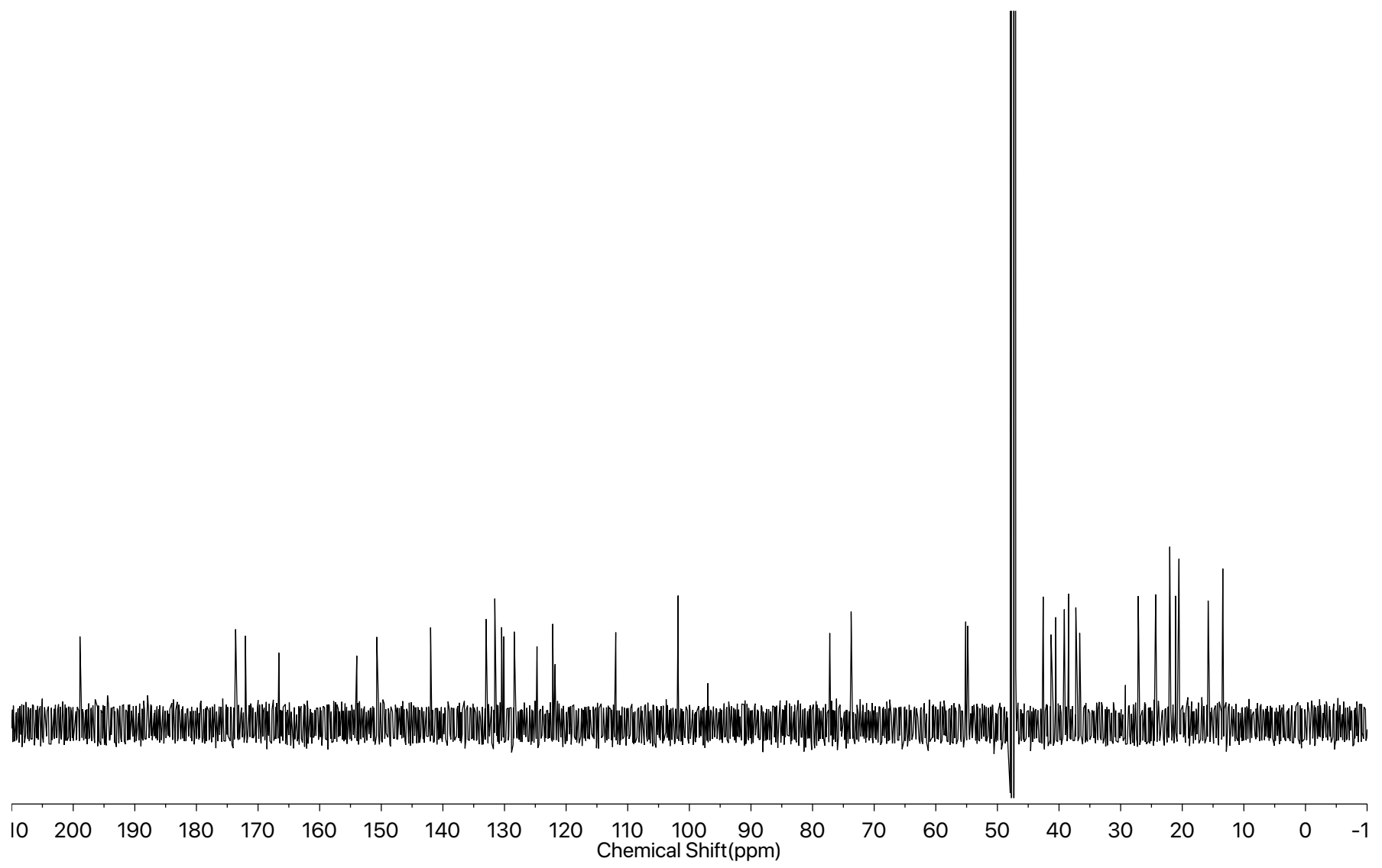




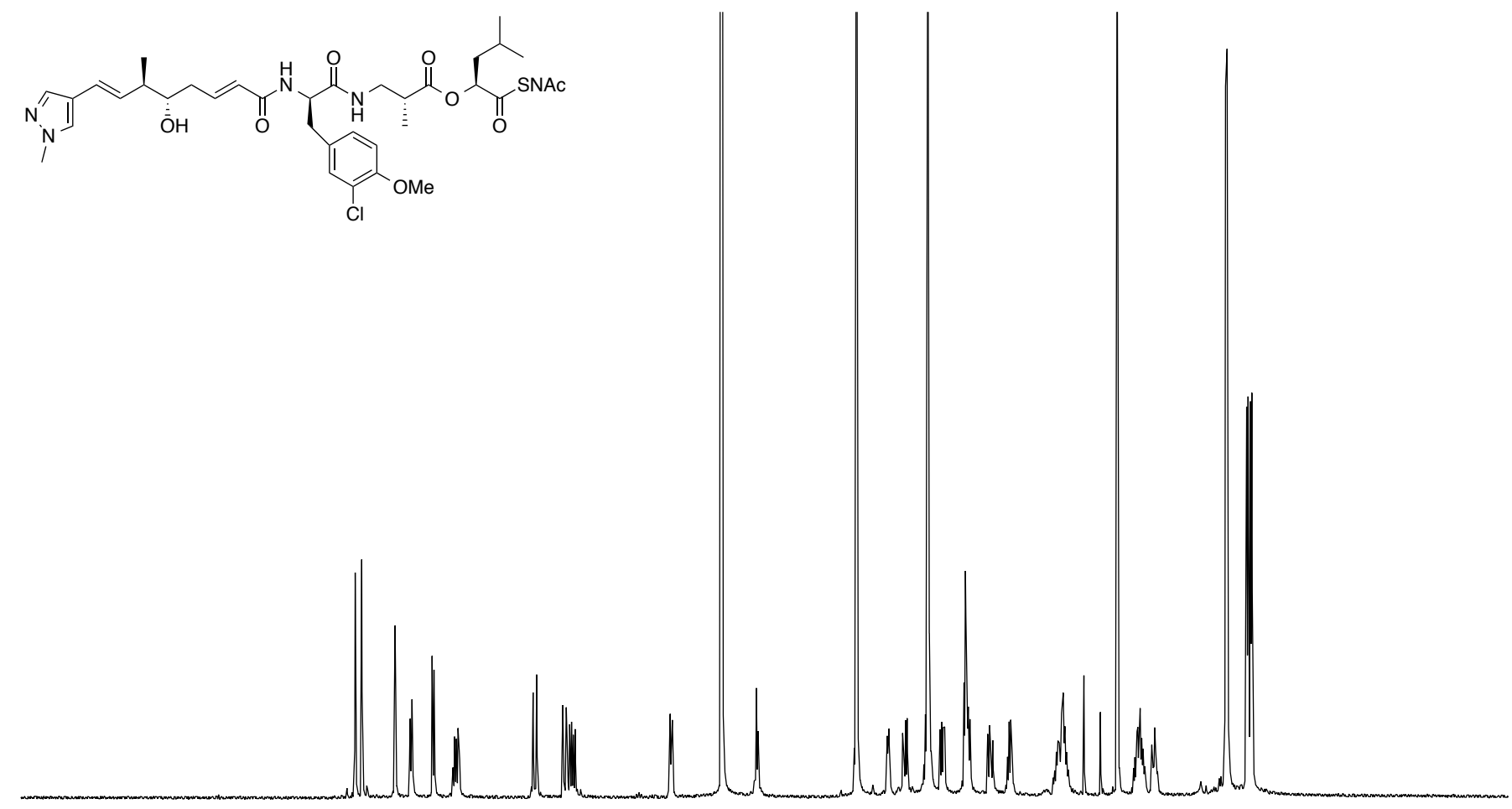

\begin{tabular}{lllllllllllllllllllll}
\hline .0 & 9.5 & 9.0 & 8.5 & 8.0 & 7.5 & 7.0 & 6.5 & 6.0 & 5.5 & $\begin{array}{c}5.0 \\
\text { Chemical Shift(ppm) }\end{array}$ & $\begin{array}{c}4.5 \\
4.0\end{array}$ & 3.0 & 2.5 & 2.0 & 1.5 & 1.0 & 0.5 & 0.0 & -0.5 & -1
\end{tabular}

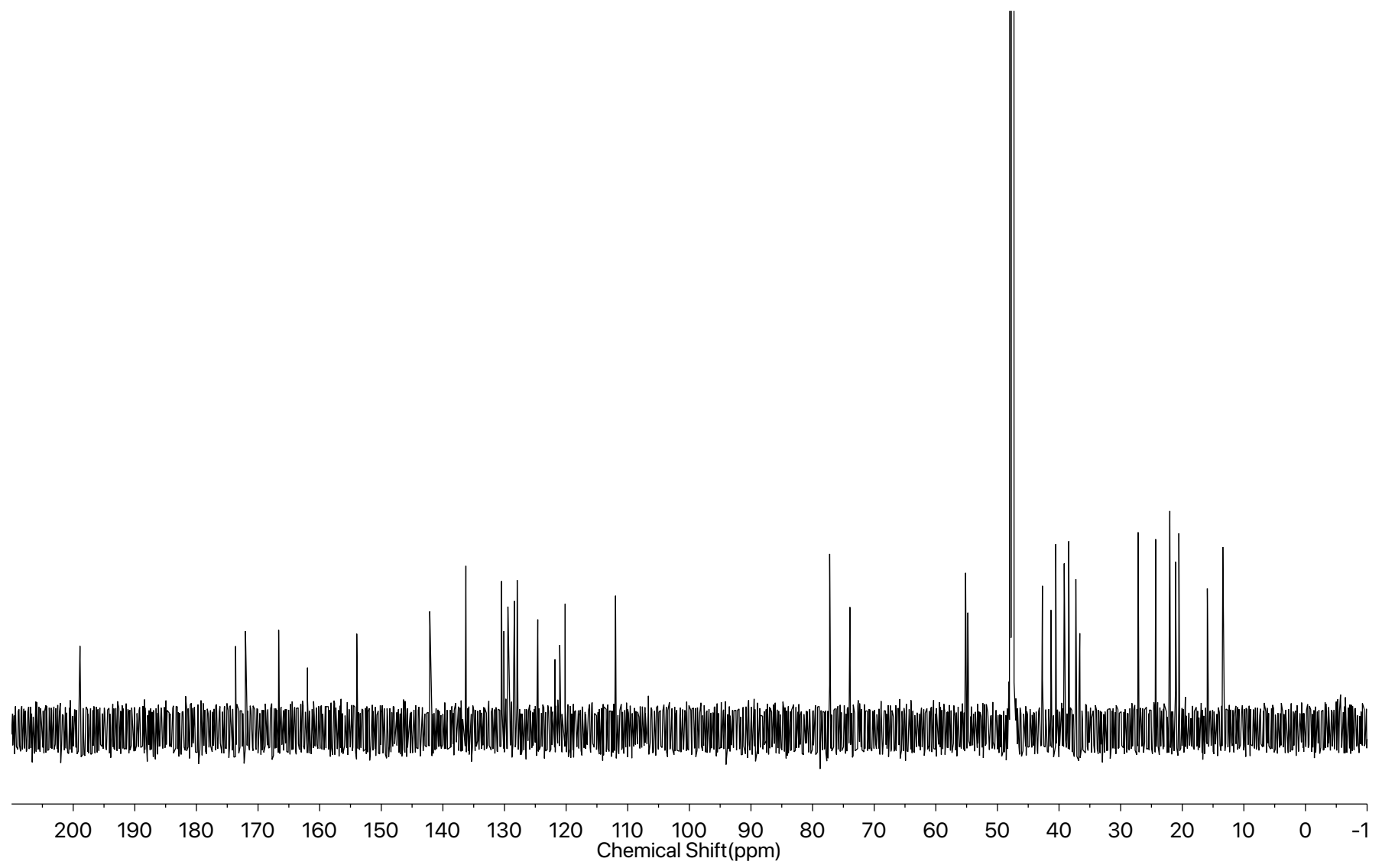



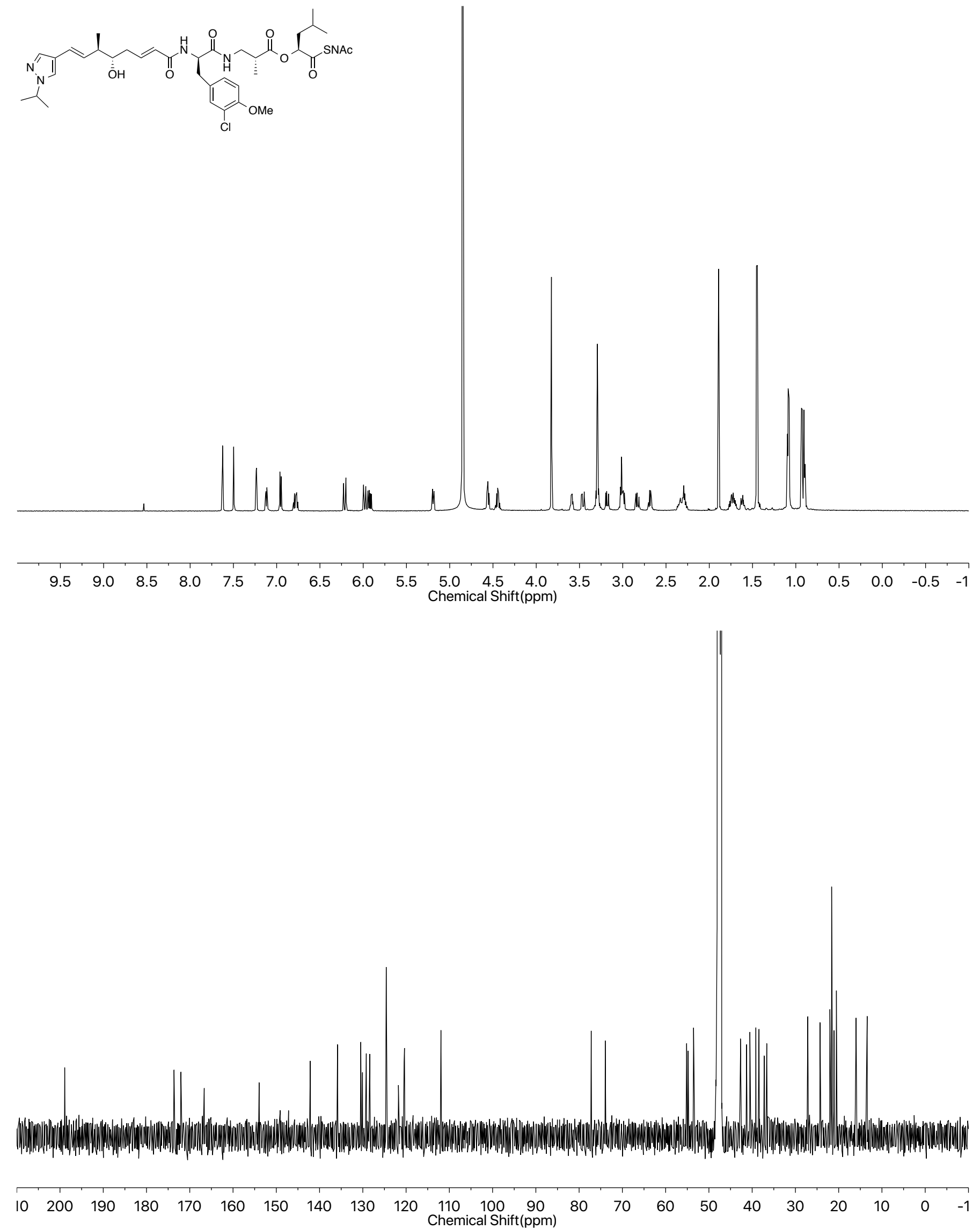

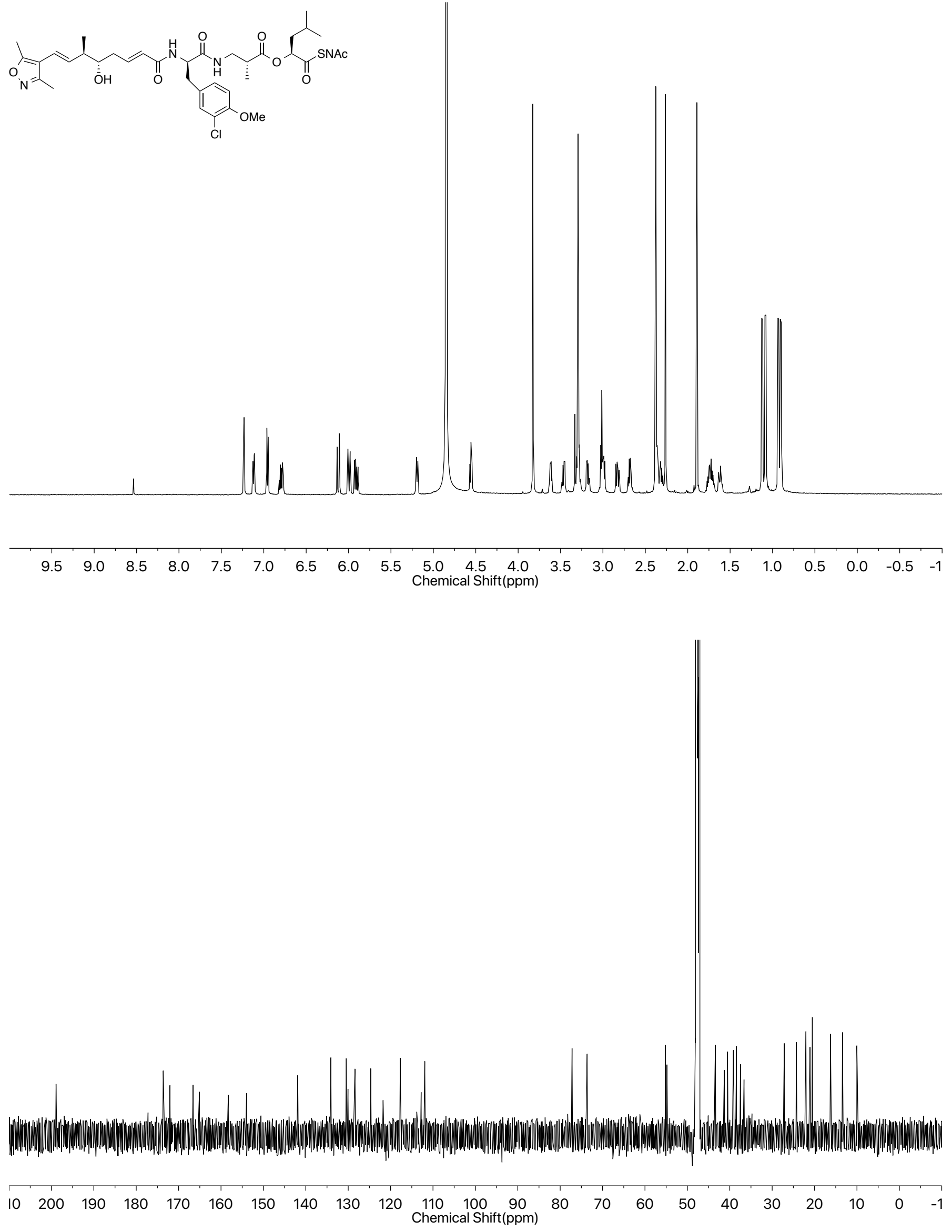


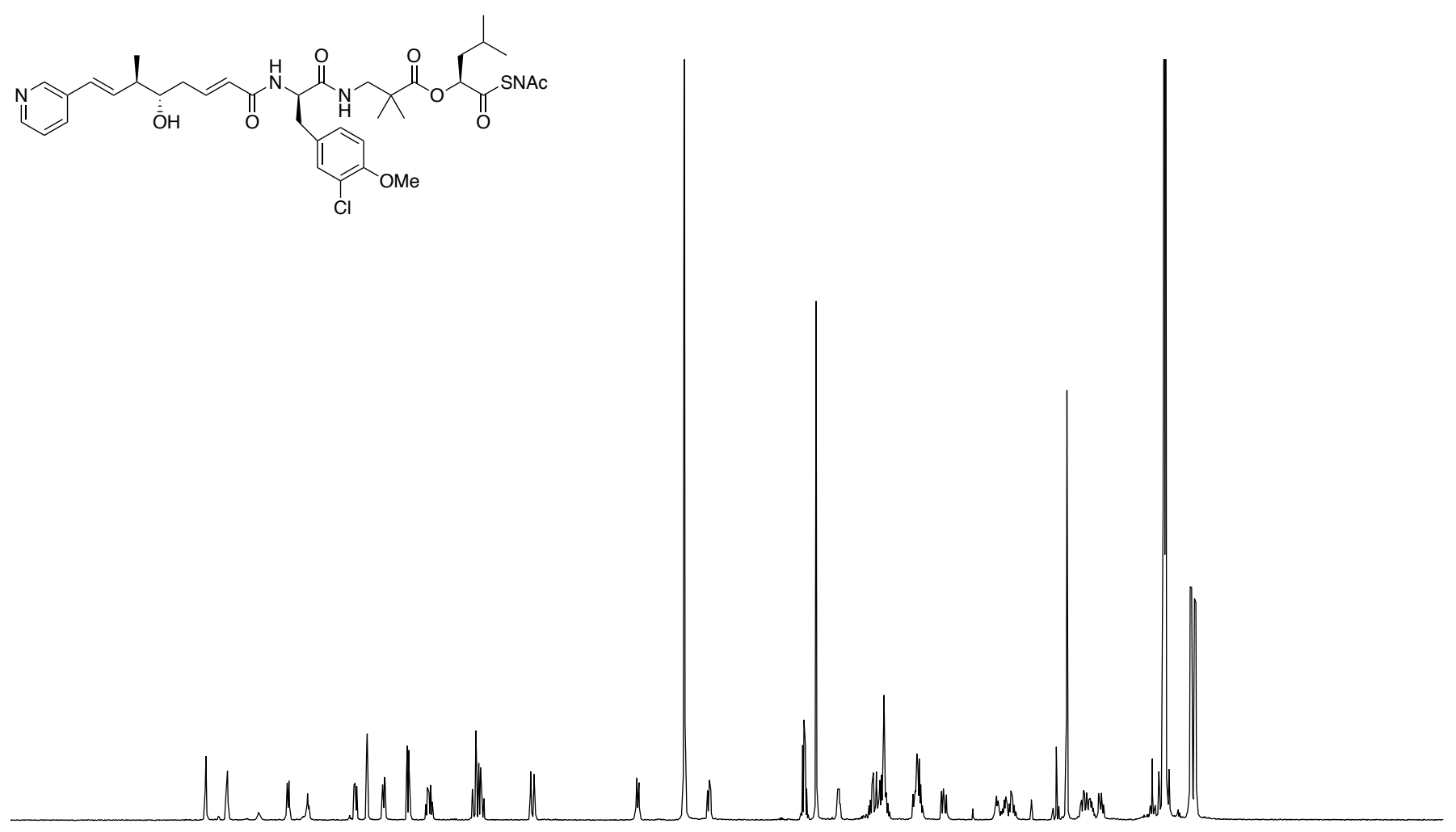

\begin{tabular}{llllllllllllllllllllll}
\hline .0 & 9.5 & 9.0 & 8.5 & 8.0 & 7.5 & 7.0 & 6.5 & 6.0 & 5.5 & $\begin{array}{c}5.0 \\
\text { Chemical Shift(ppm) }\end{array}$ & 4.5 & 3.0 & 2.5 & 2.0 & 1.5 & 1.0 & 0.5 & 0.0 & -0.5 & -1
\end{tabular}

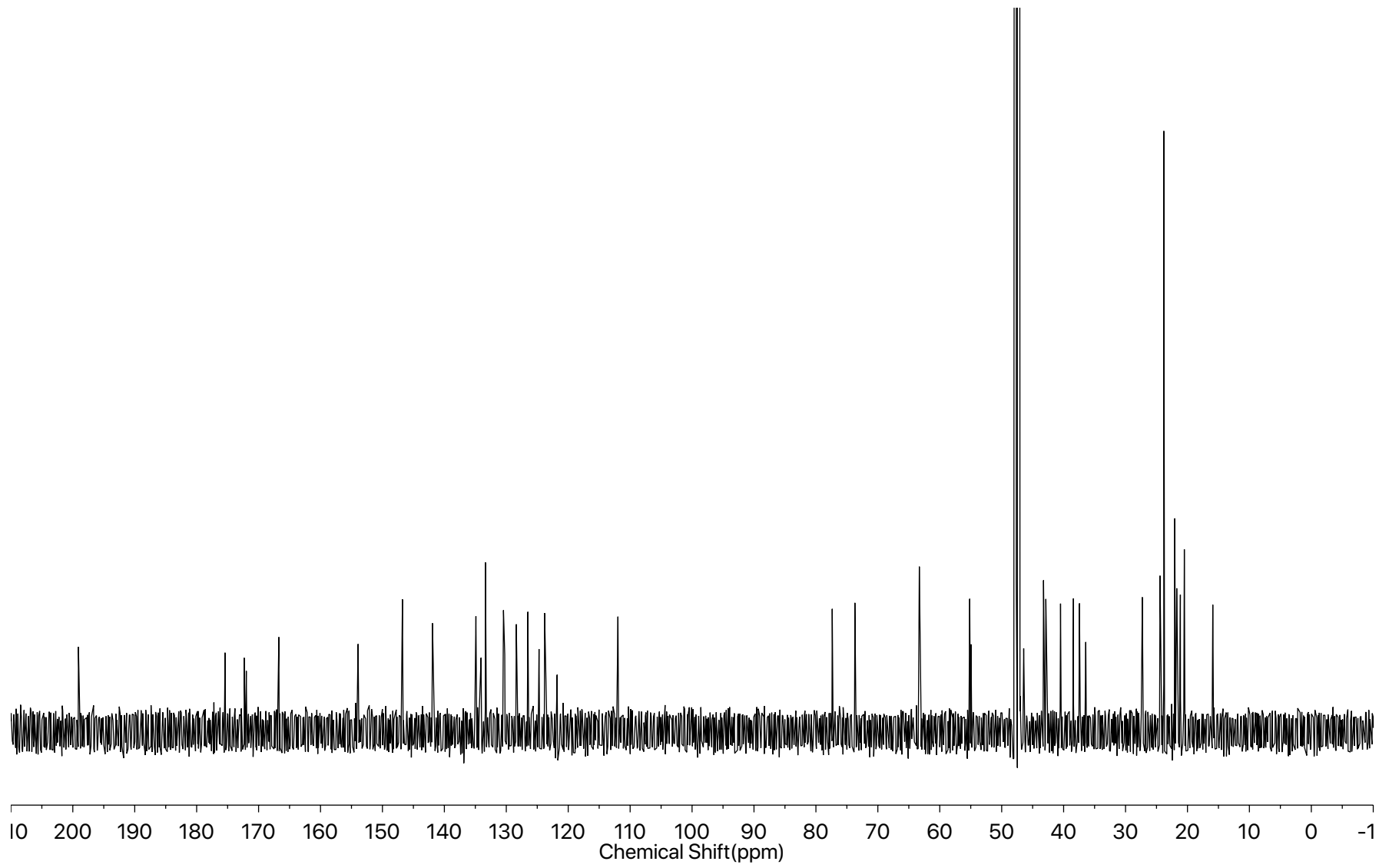



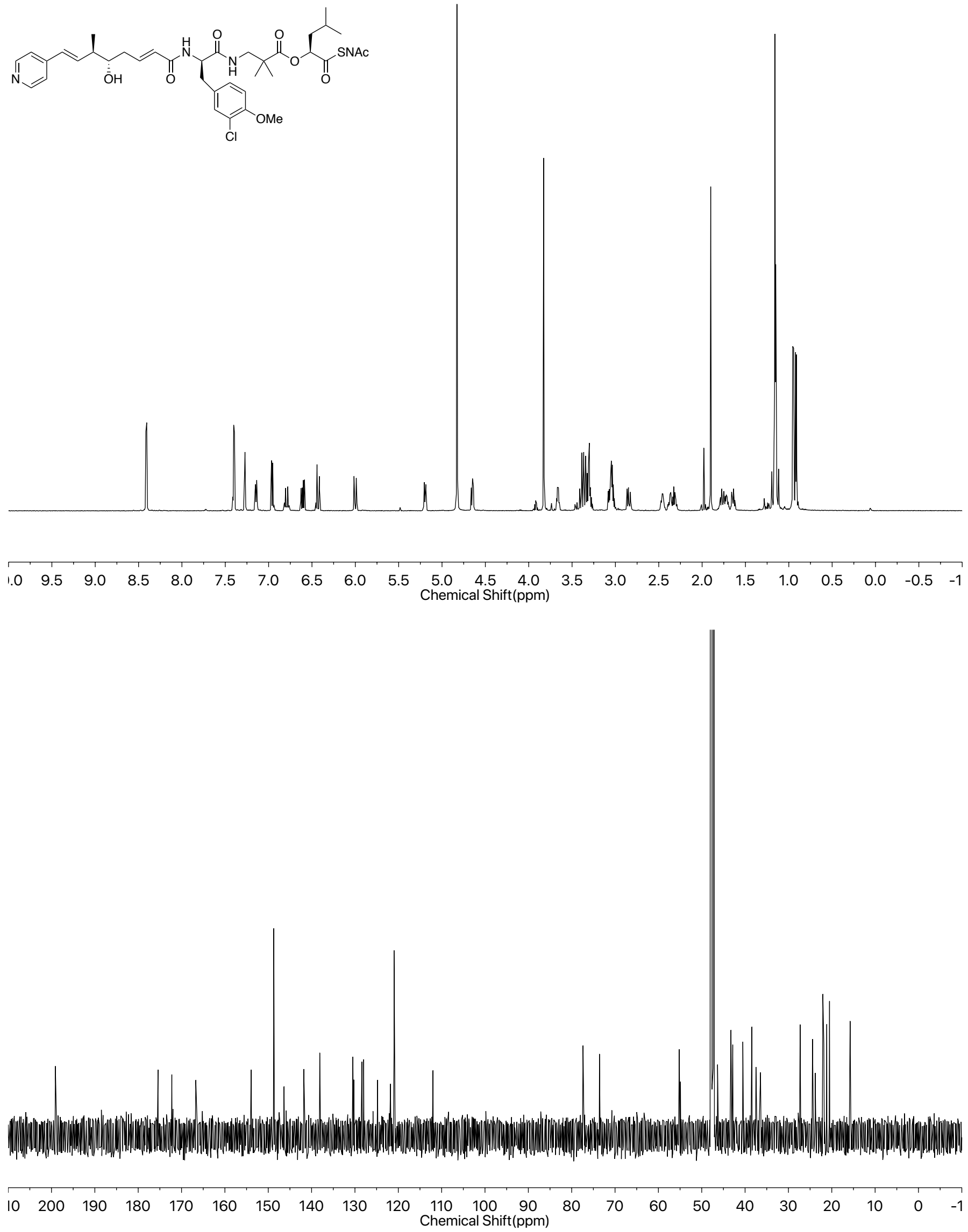

S63 


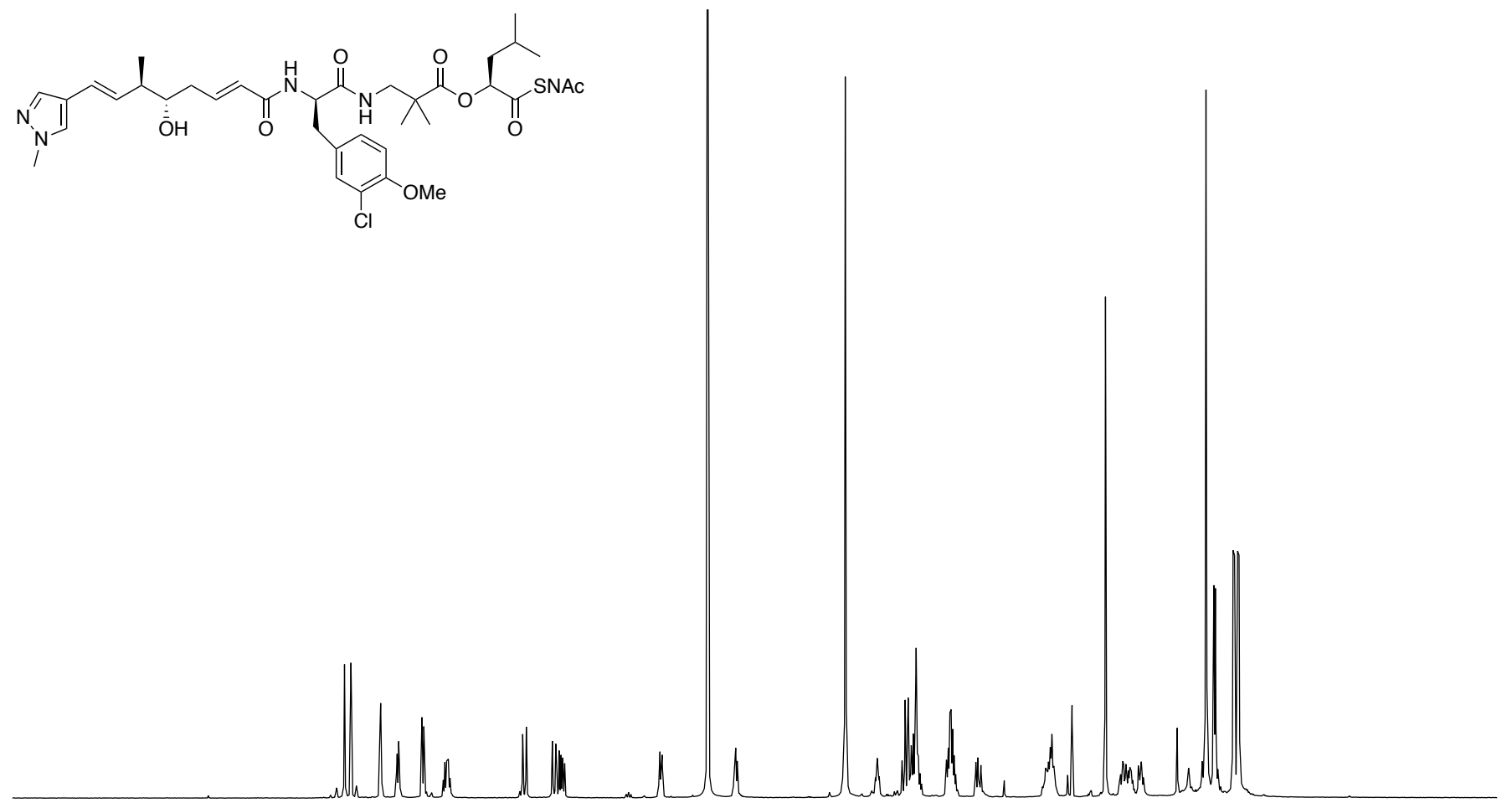

\begin{tabular}{lllllllllllllllllllll}
\hline .0 & 9.5 & 9.0 & 8.5 & 8.0 & 7.5 & 7.0 & 6.5 & 6.0 & 5.5 & $\begin{array}{c}5.0 \\
\text { Chemical Shift(ppm) }\end{array}$ & 4.5 & 3.0 & 2.5 & 2.0 & 1.5 & 1.0 & 0.5 & 0.0 & -0.5 & -1
\end{tabular}
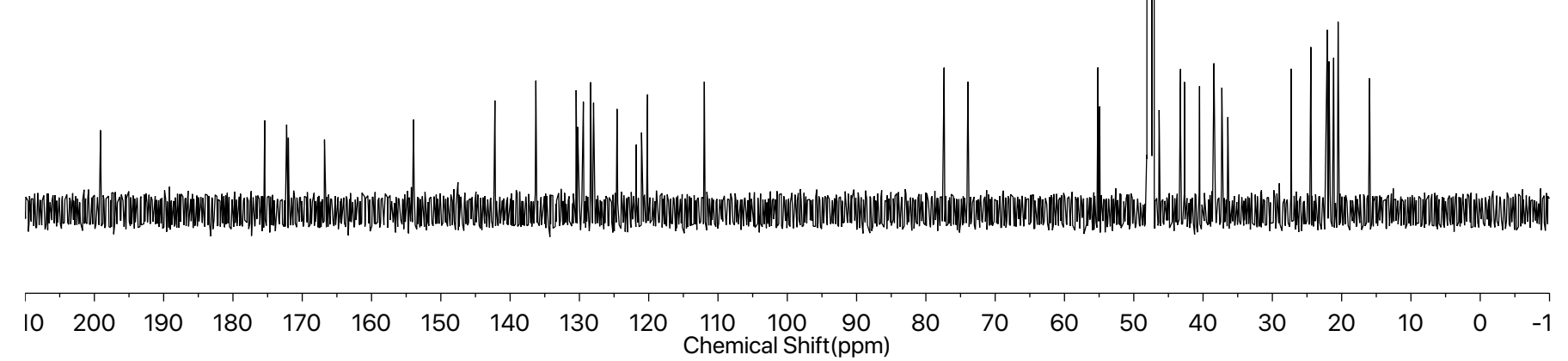

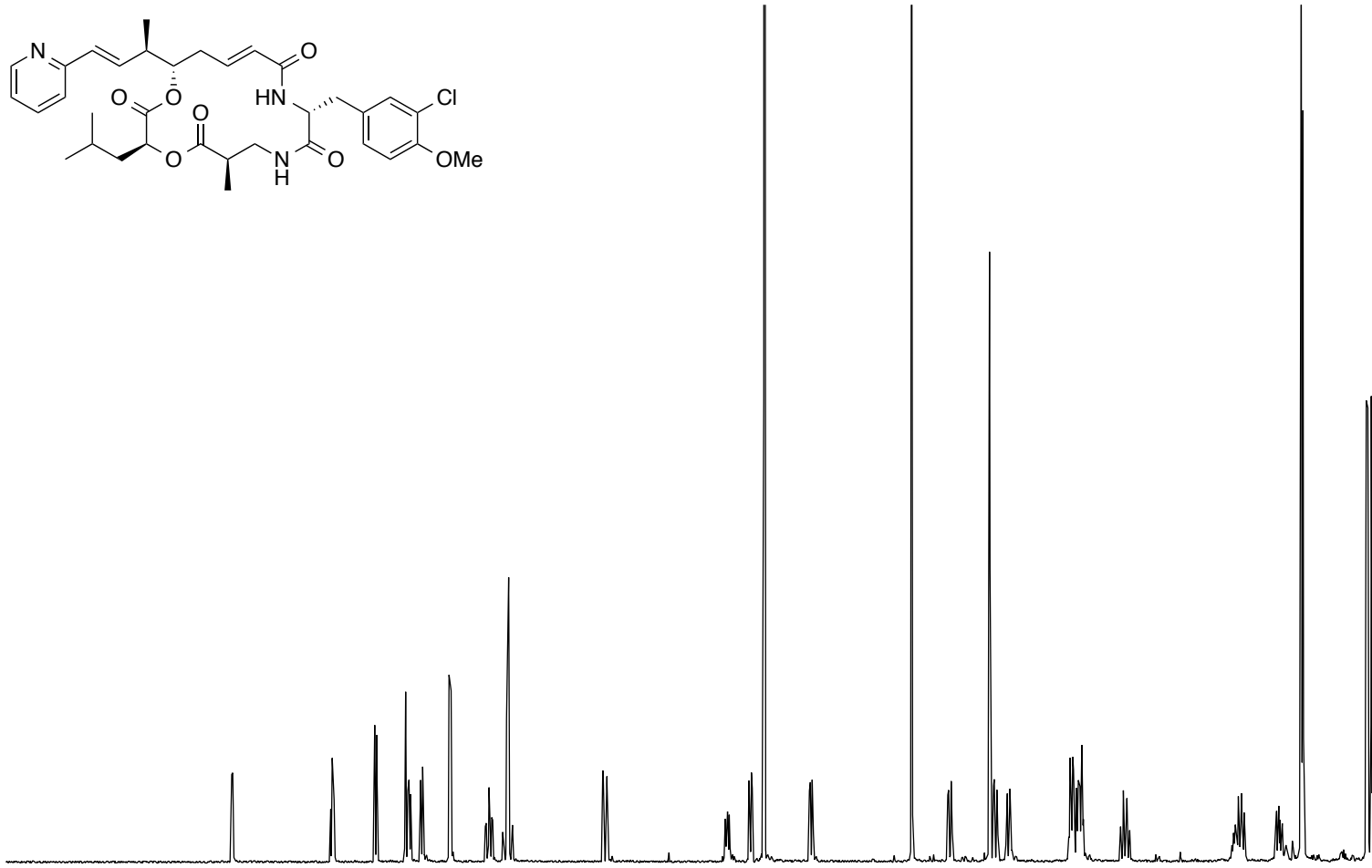

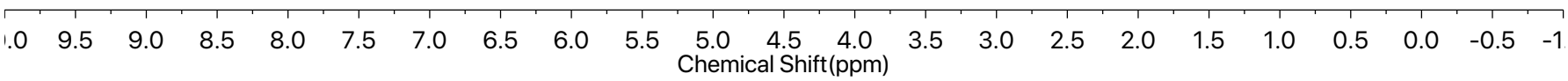

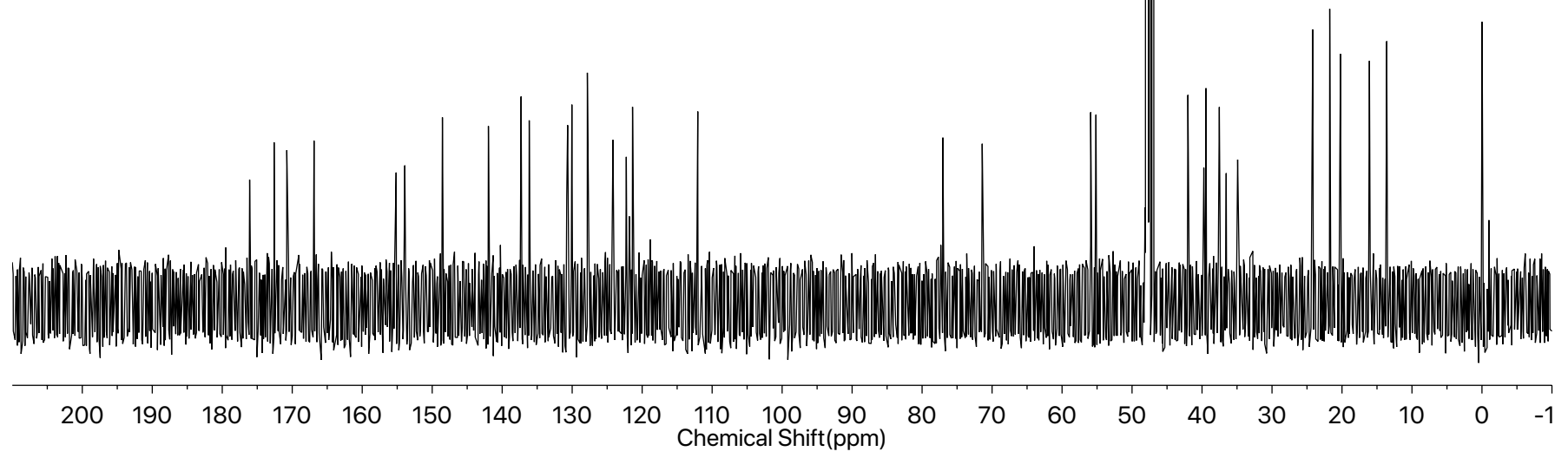


<smiles>COc1ccc(C[C@H](NC(=O)/C=C/CC(C)C(C)/C=C/c2ccncn2)C(=O)OC(=O)C(CC(C)C)OC(=O)C(C)CC(C)C)cc1Cl</smiles>

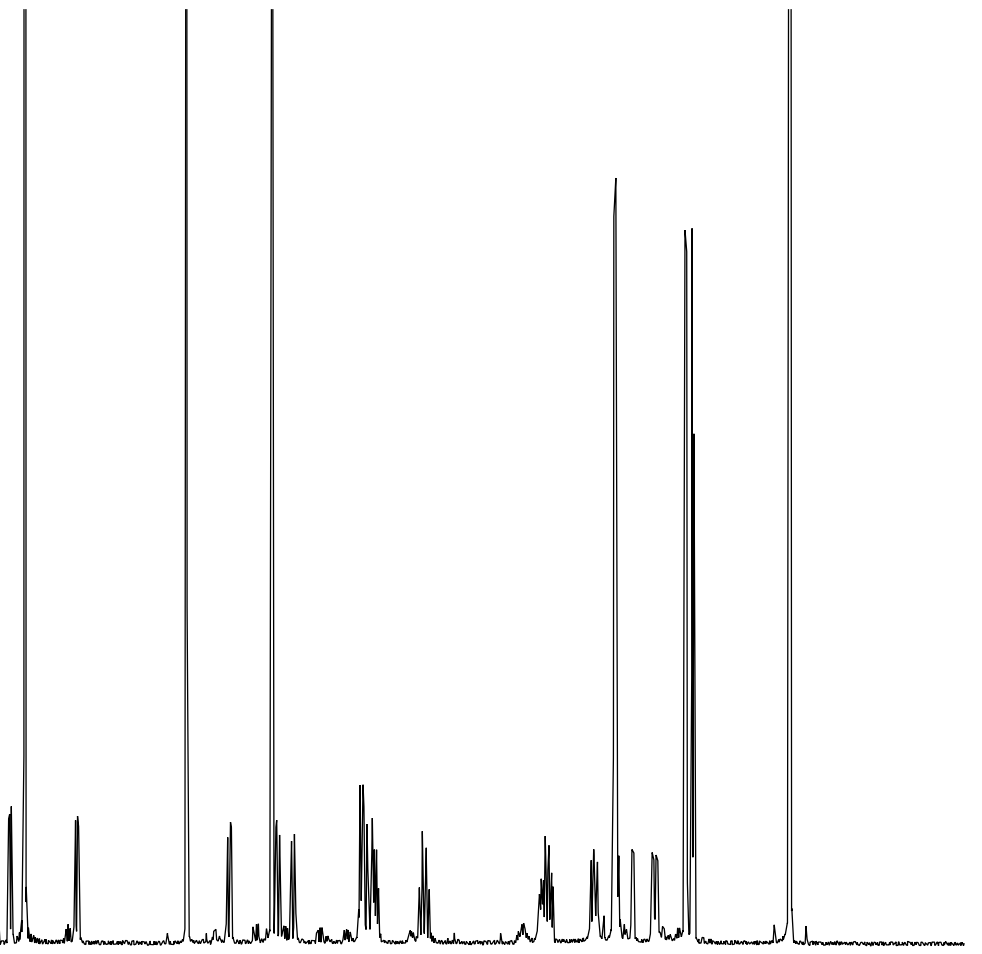

$\begin{array}{lllllllllllllllllllllll}1.0 & 9.5 & 9.0 & 8.5 & 8.0 & 7.5 & 7.0 & 6.5 & 6.0 & 5.5 & \begin{array}{c}5.0 \\ \text { Chemical Shift(ppm) }\end{array} & \begin{array}{l}4.5 \\ 4.0\end{array} & 3.0 & 2.5 & 2.0 & 1.5 & 1.0 & 0.5 & 0.0 & -0.5 & -1\end{array}$ *minor peaks represent inseperable diastereomers

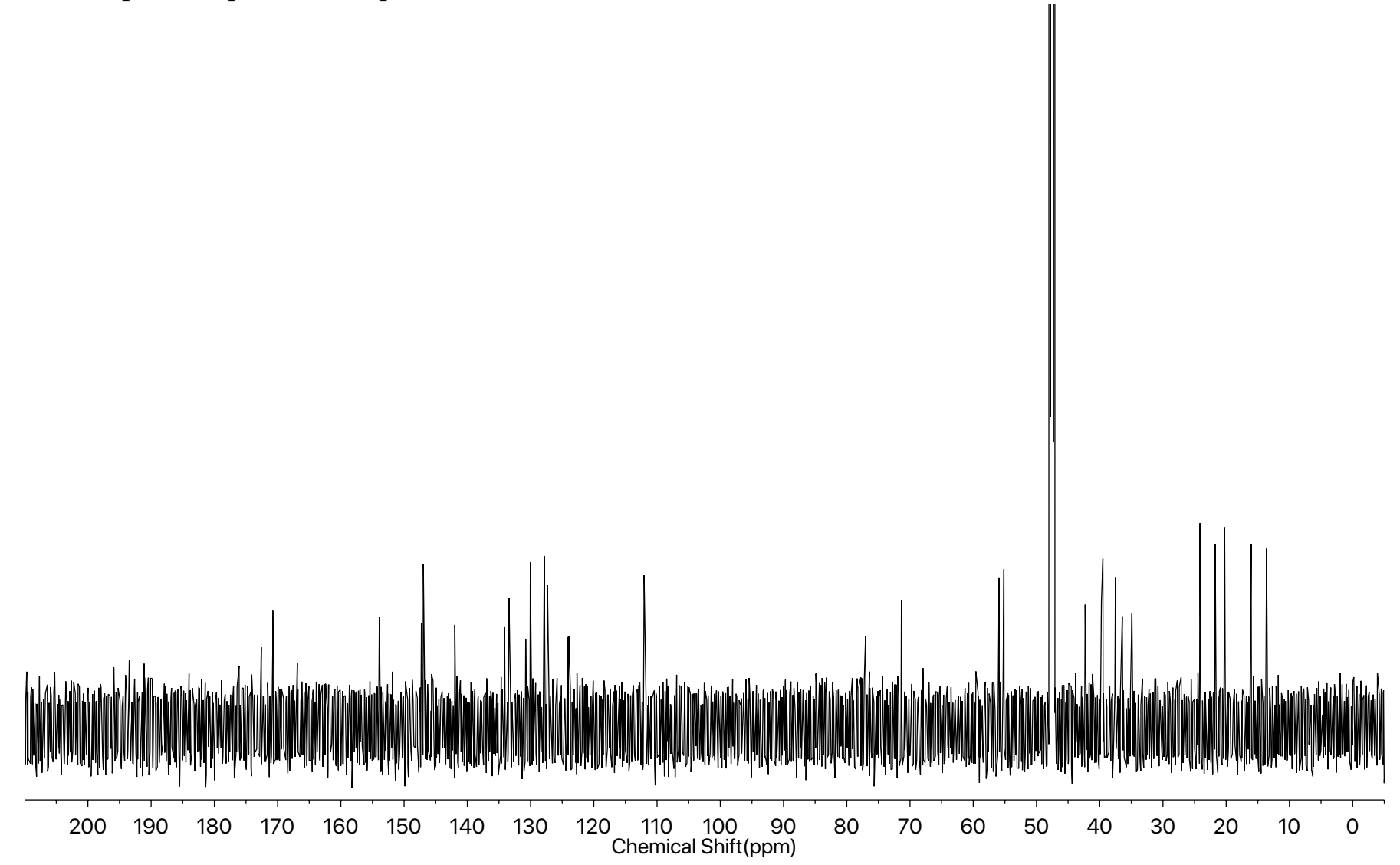



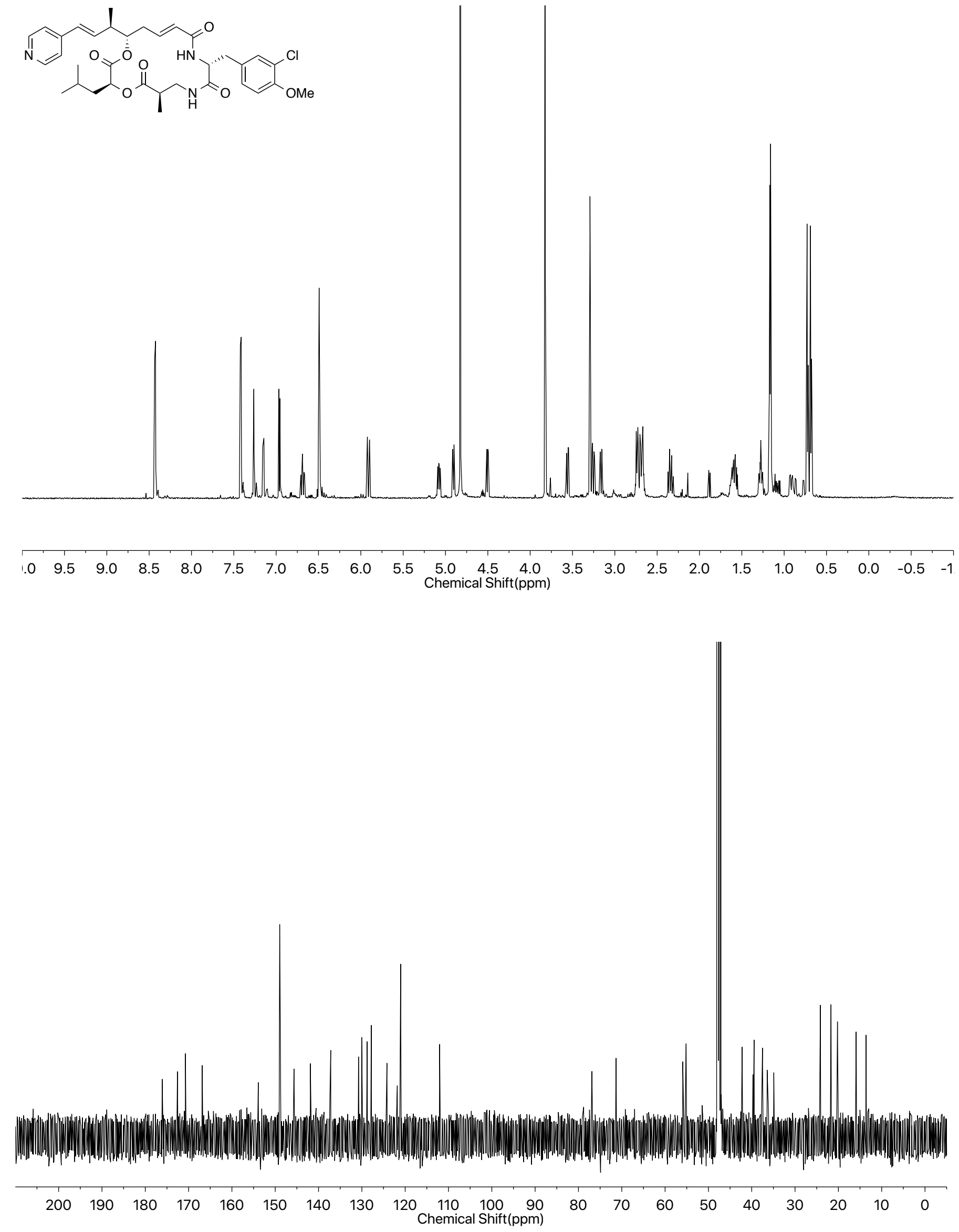
<smiles>COc1ccc(C[C@H](NC(=O)/C=C/C[C@H](OC(=O)C(CC(C)C)OC(=O)C(C)CNC(=O)[C@H](C)/C=C/c2cnccn2)C(C)C)C(=O)O)cc1Cl</smiles>
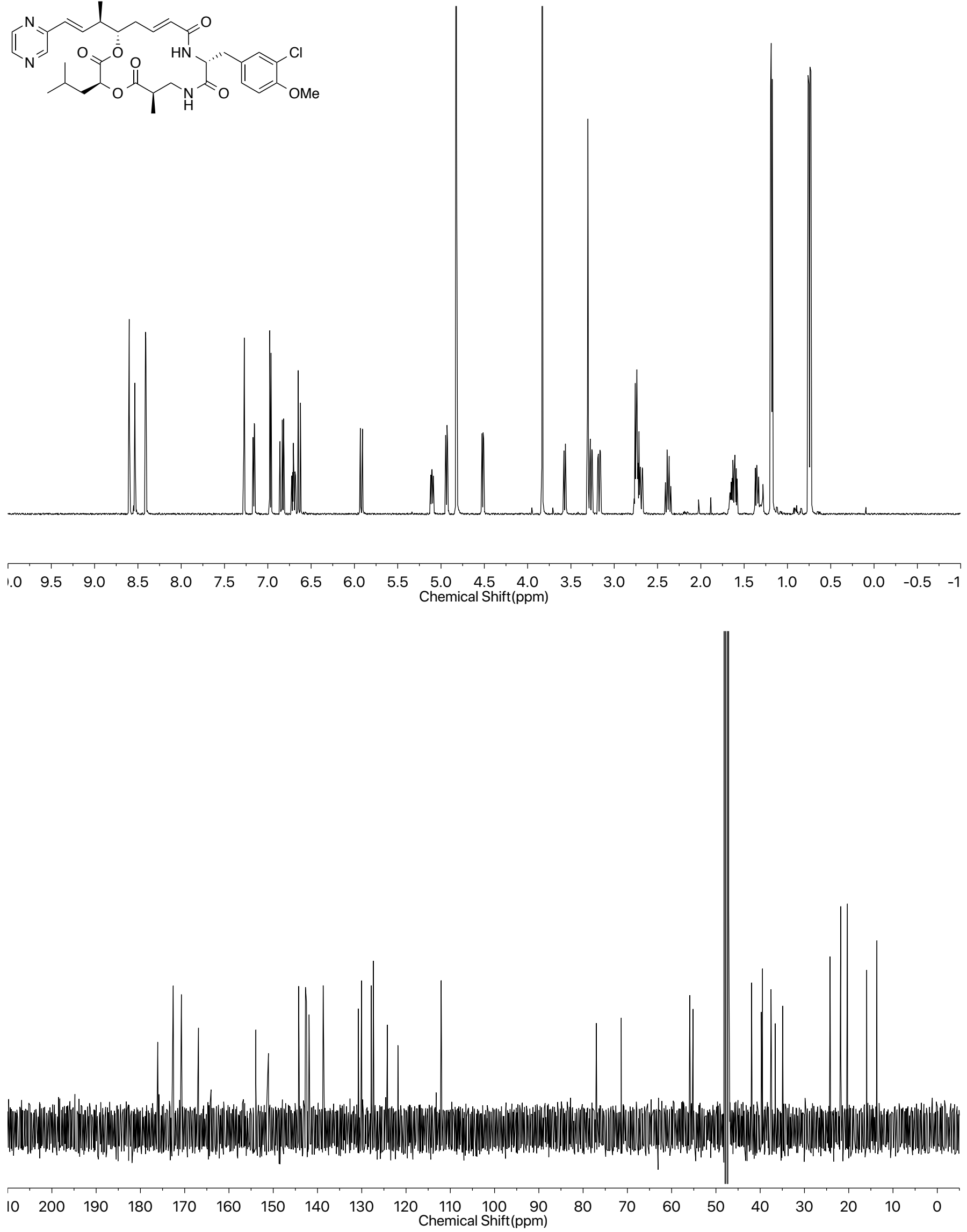

S68 

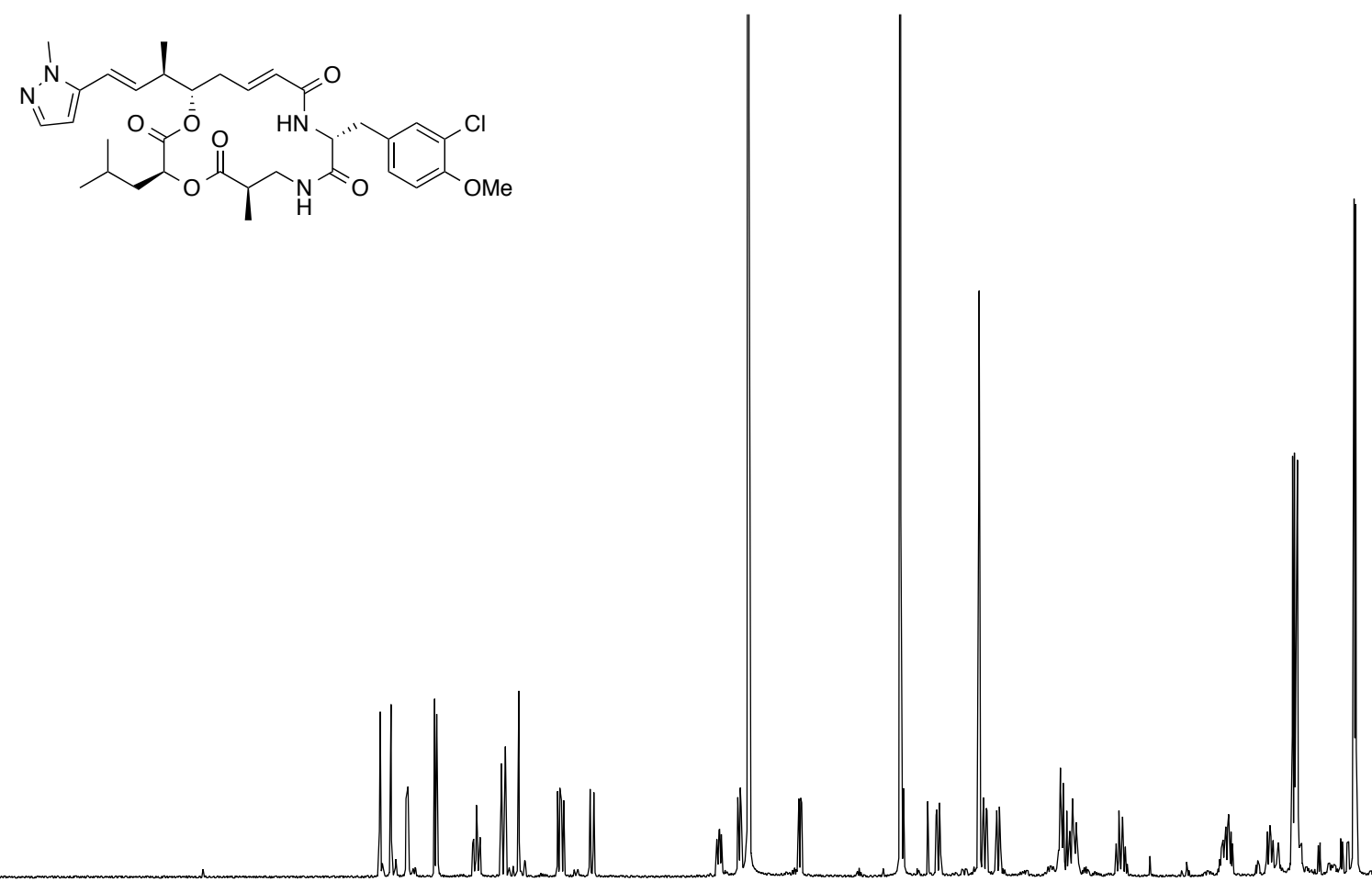

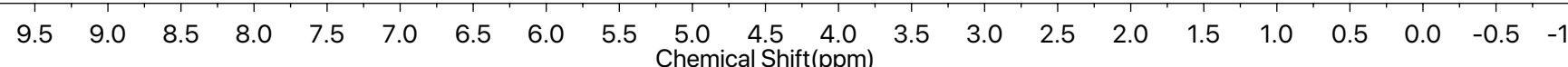

*minor peaks represent inseperable diastereomers

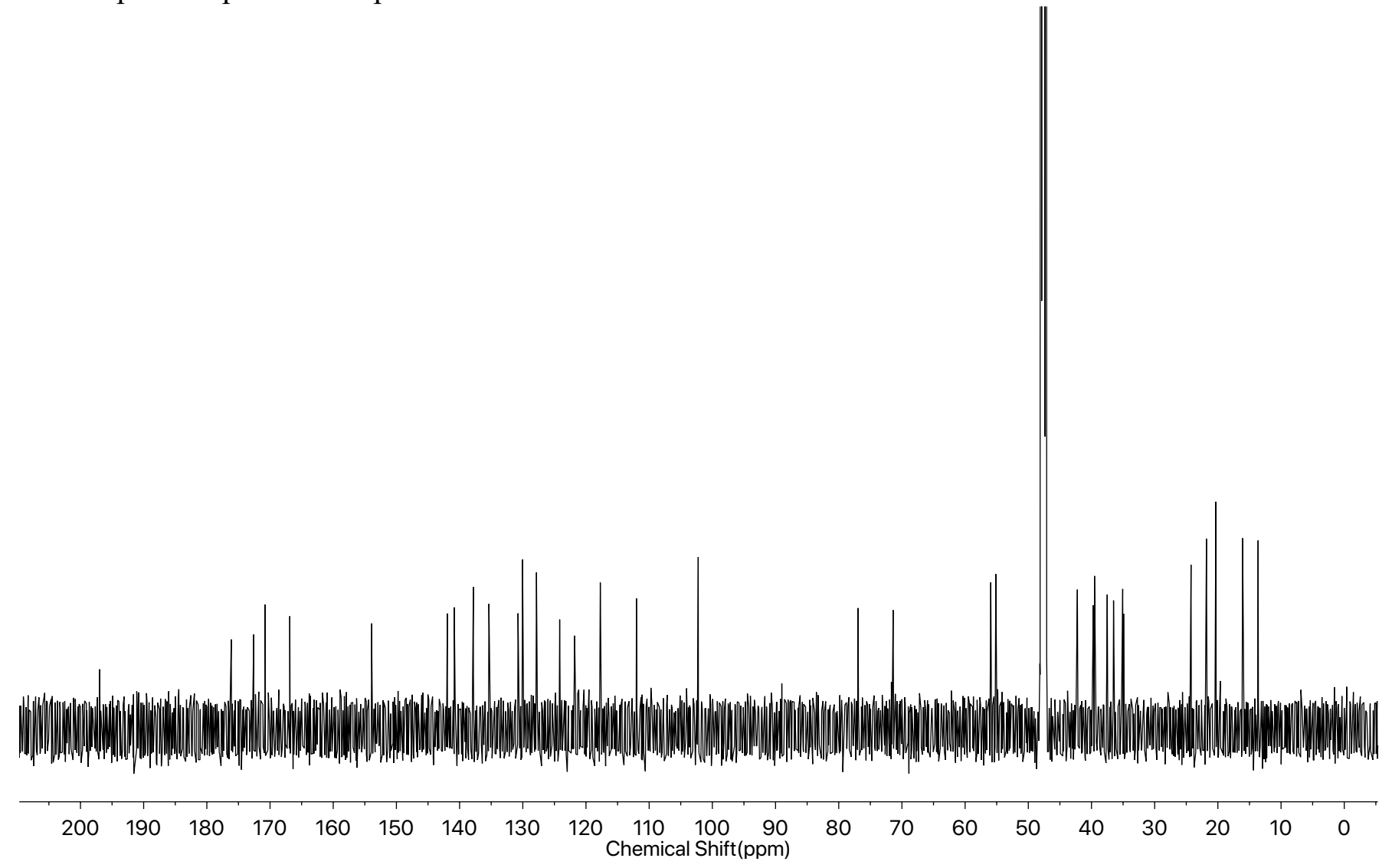




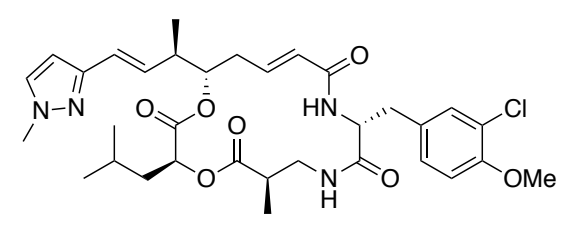

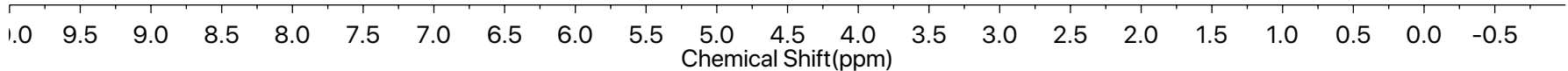

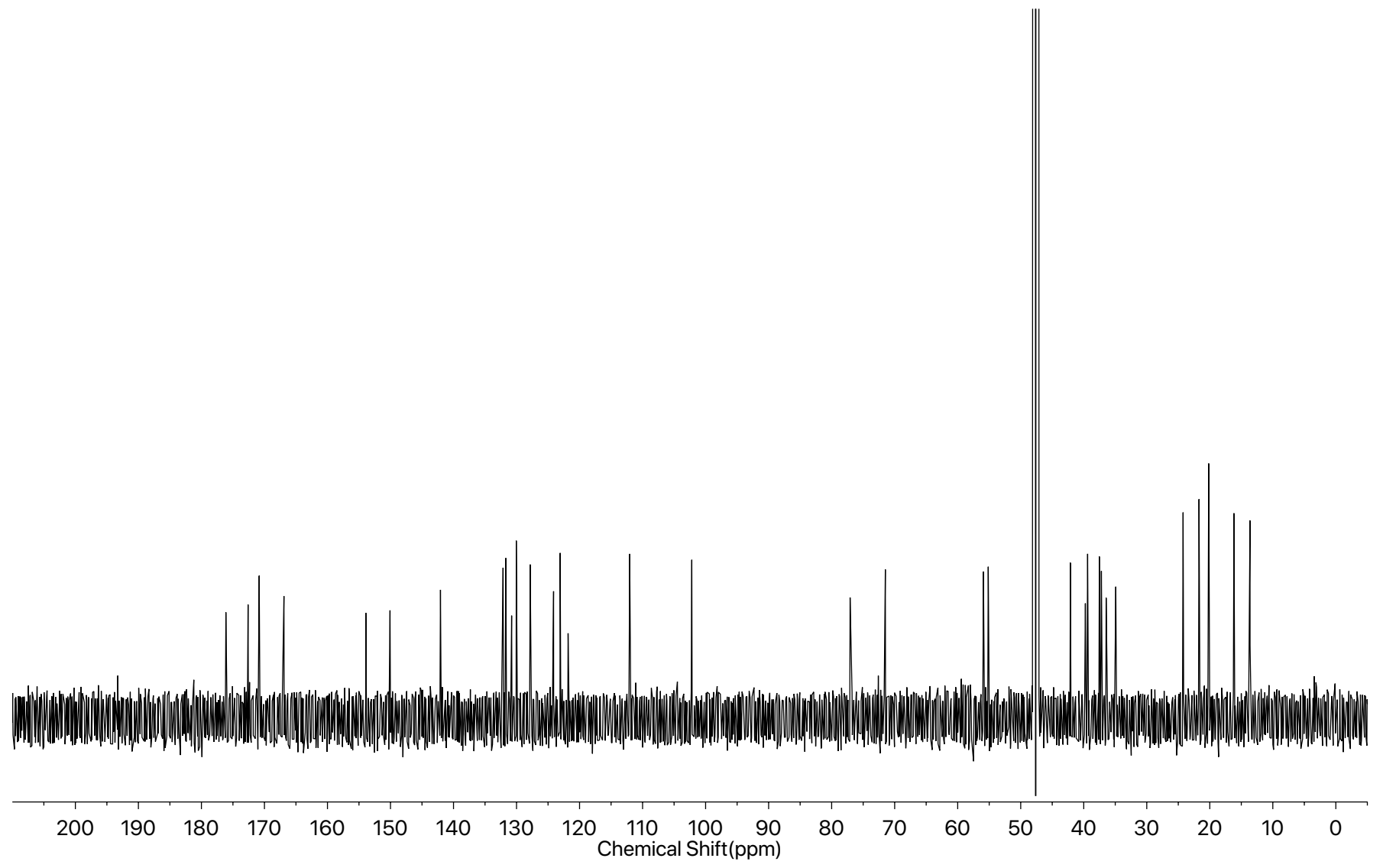



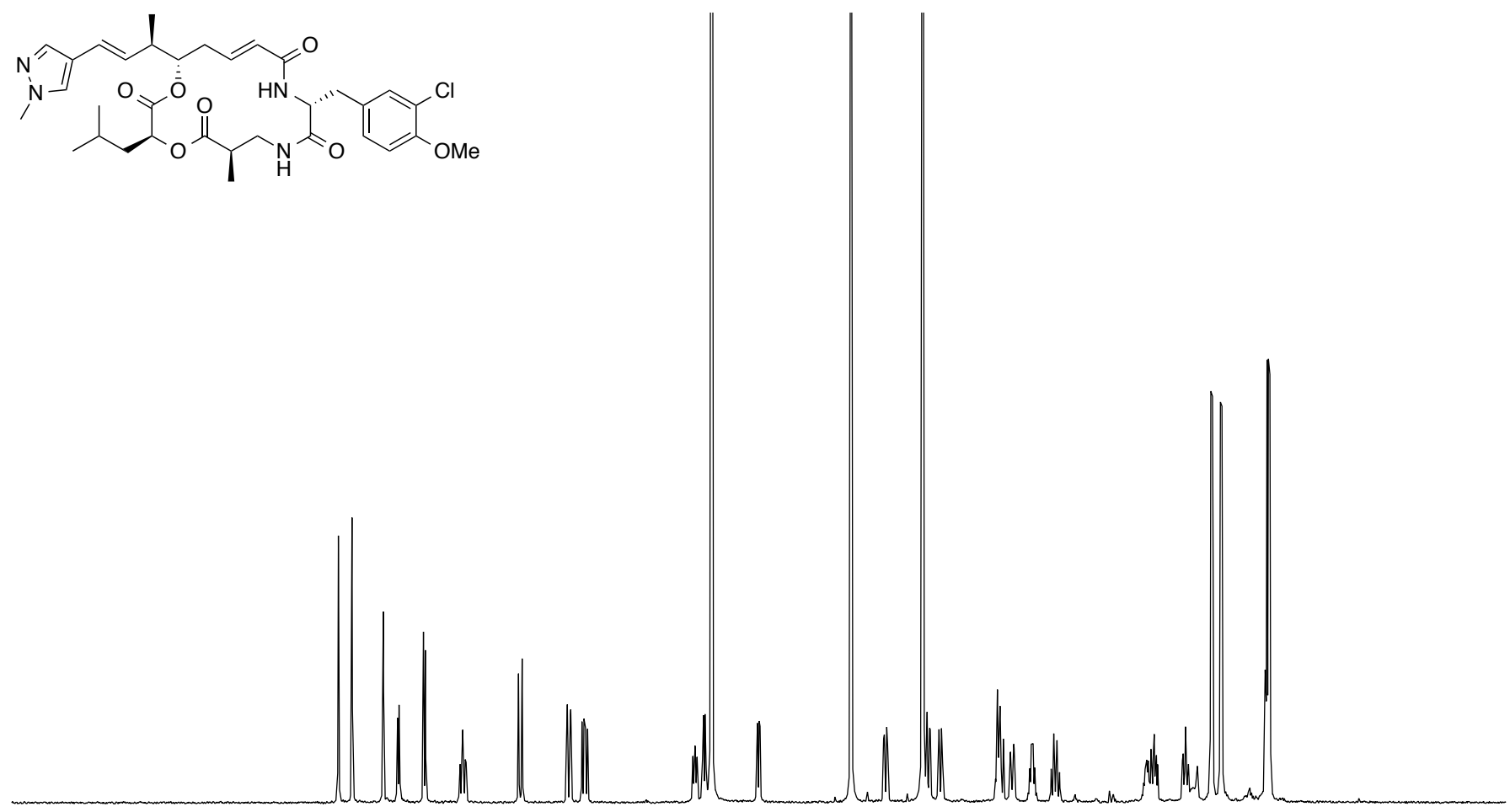

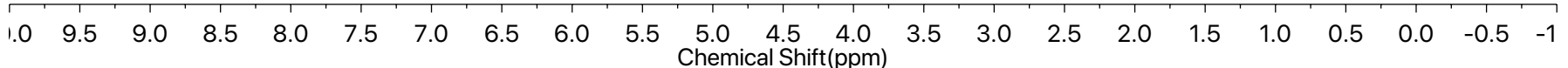

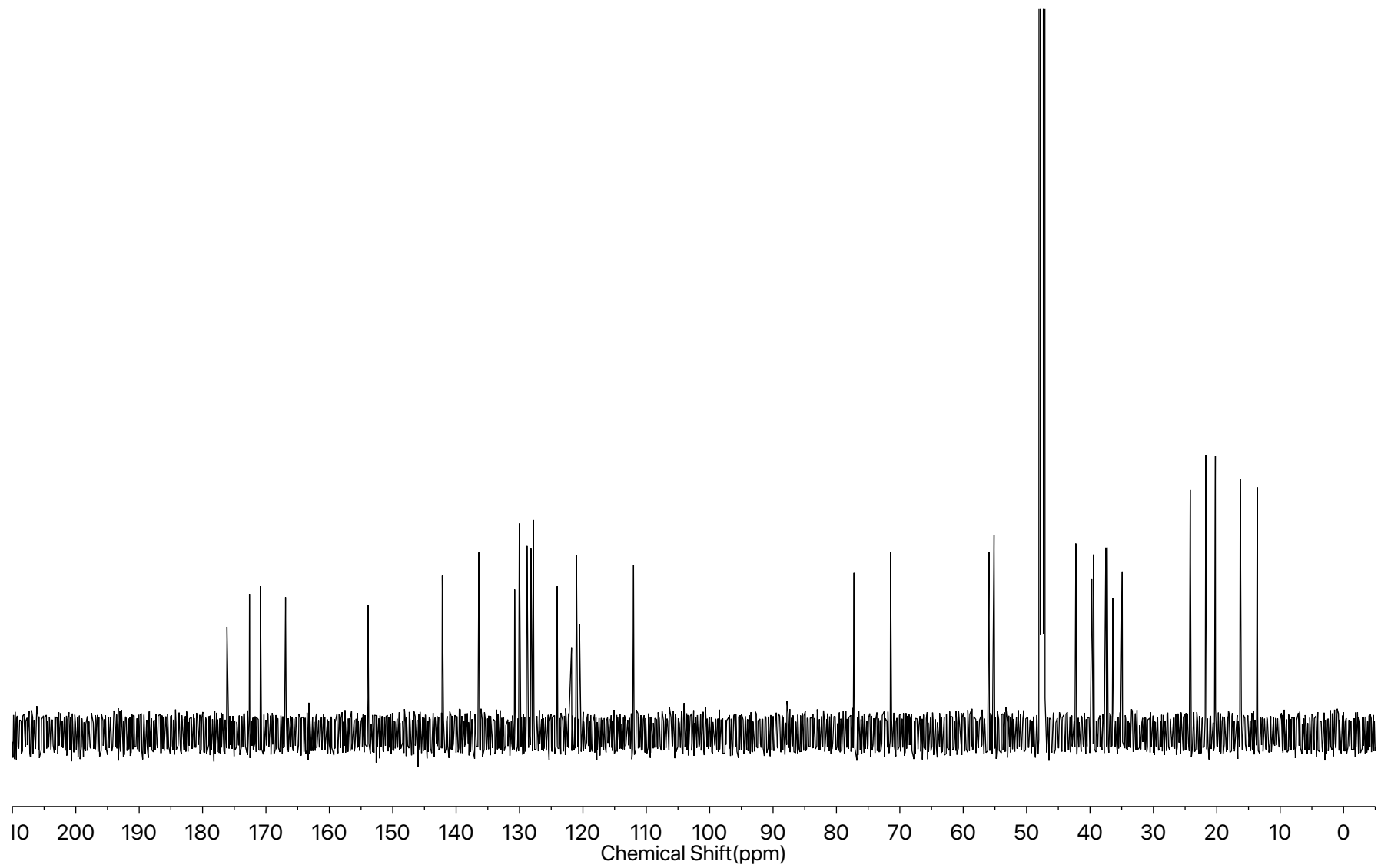



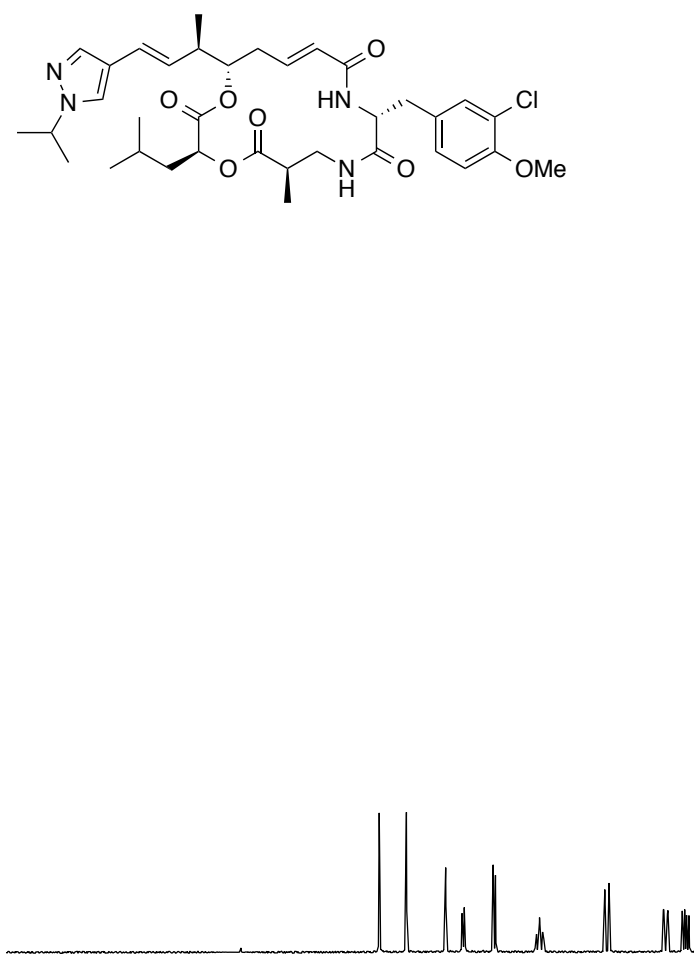

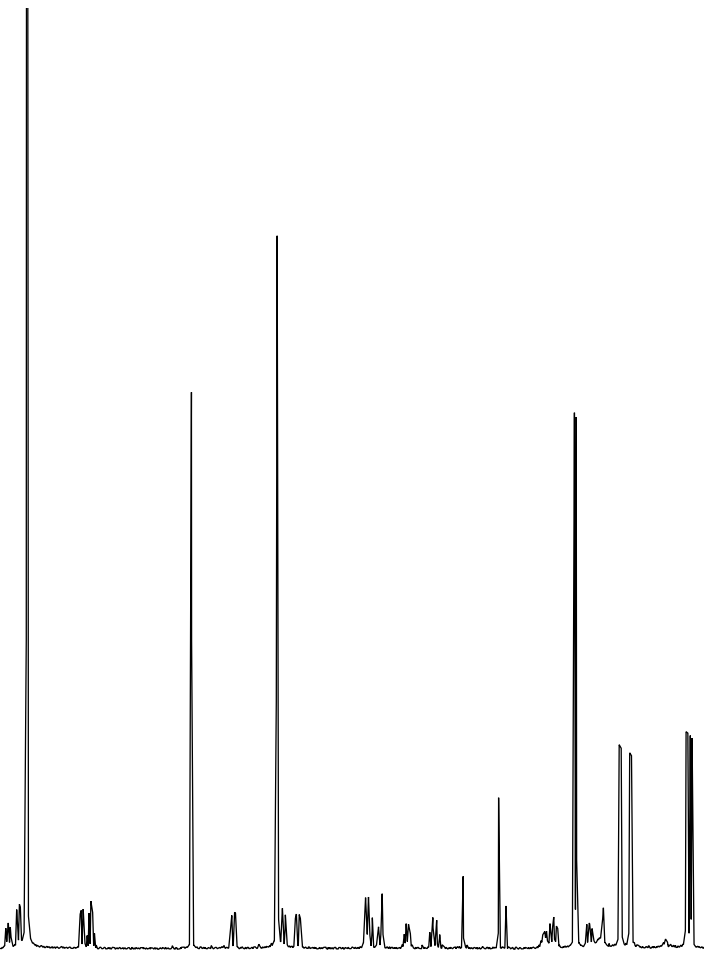

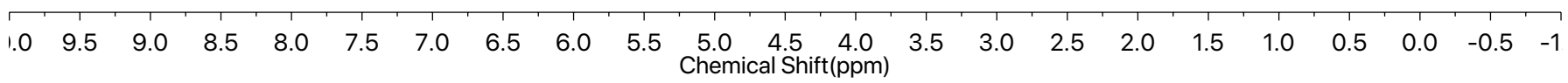

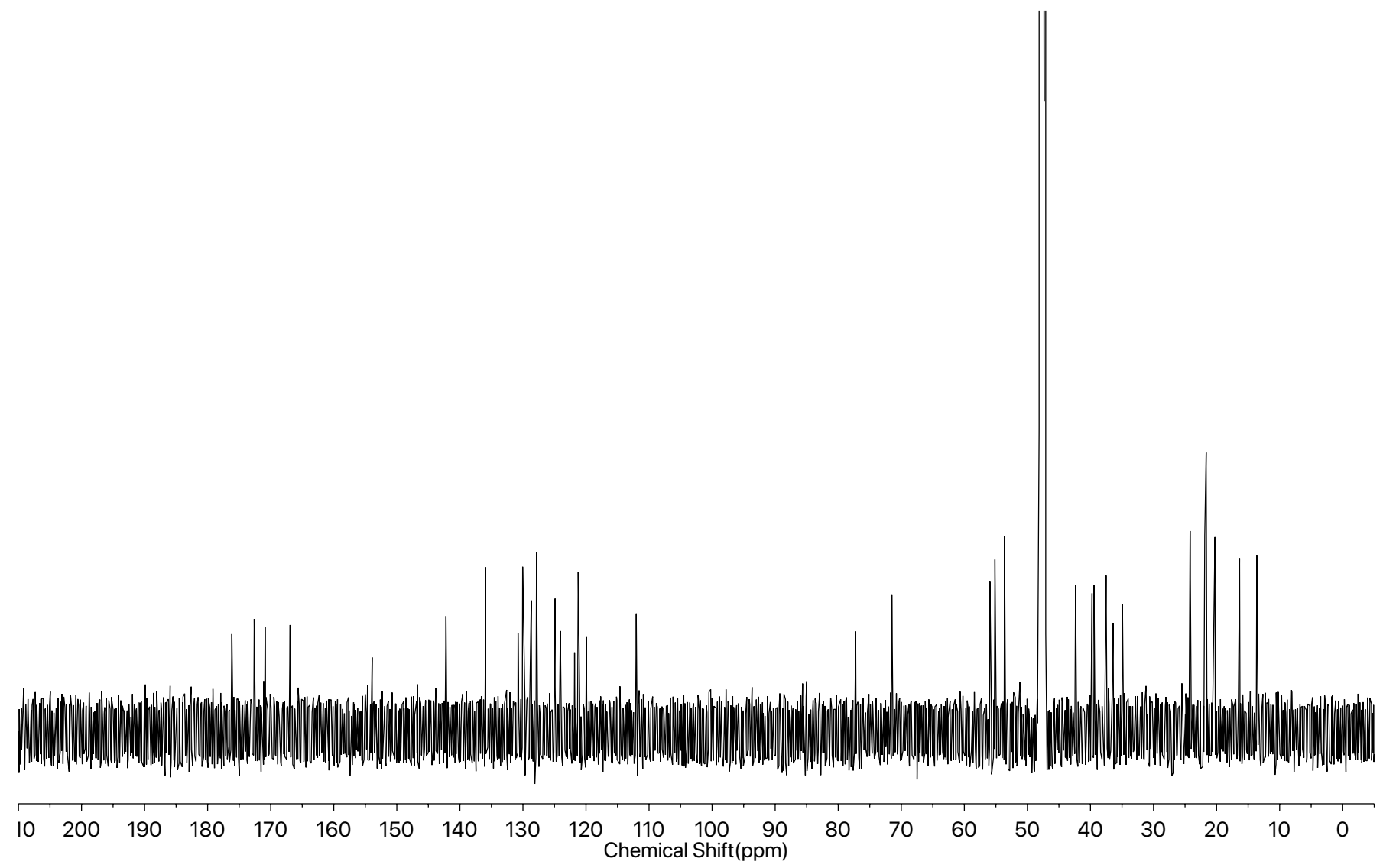

S72 


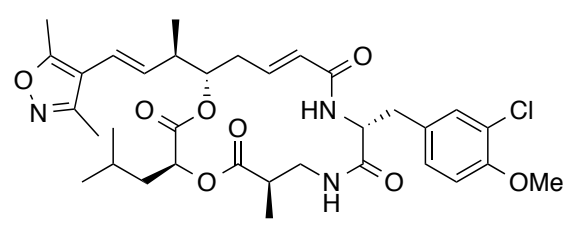
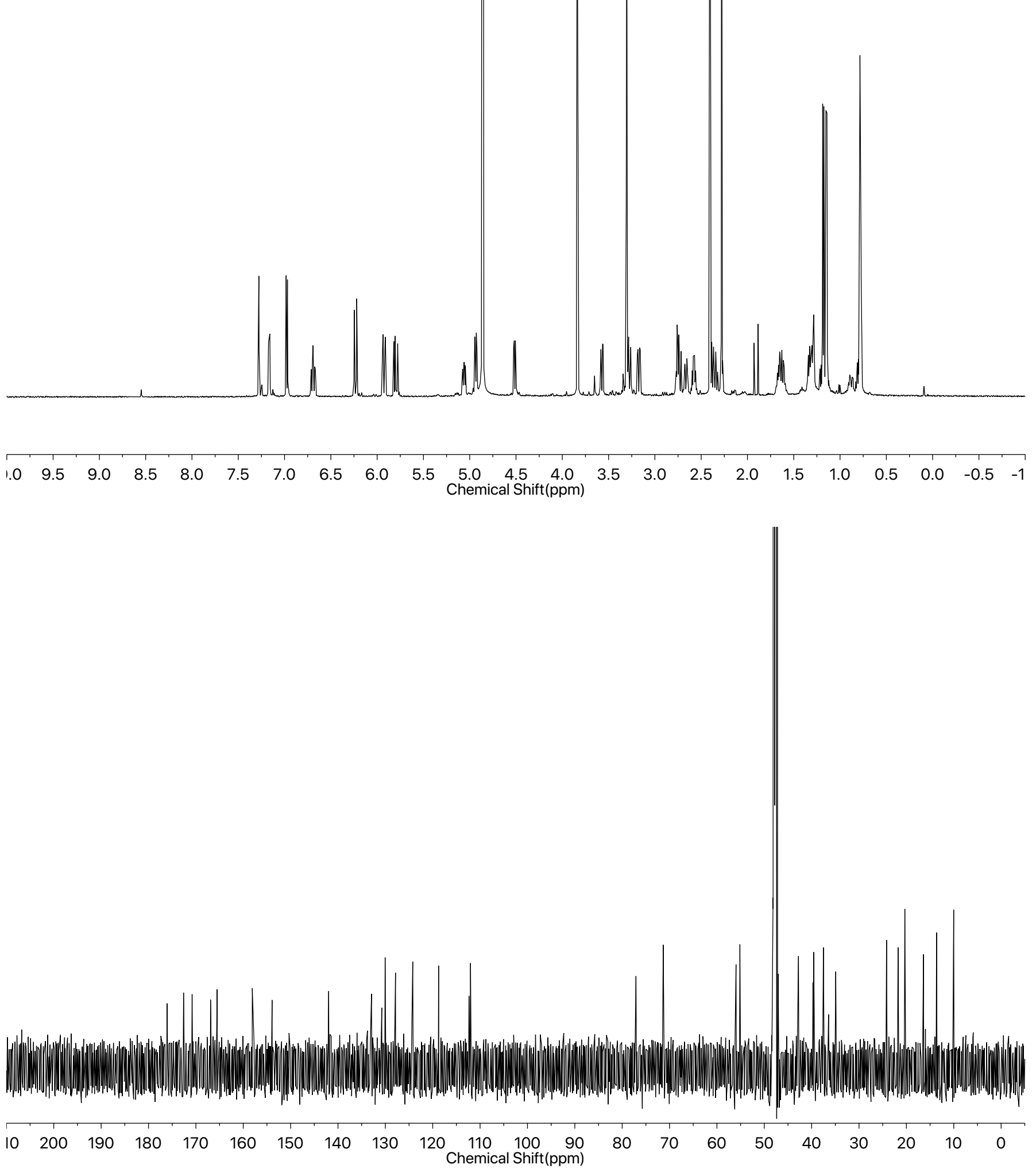

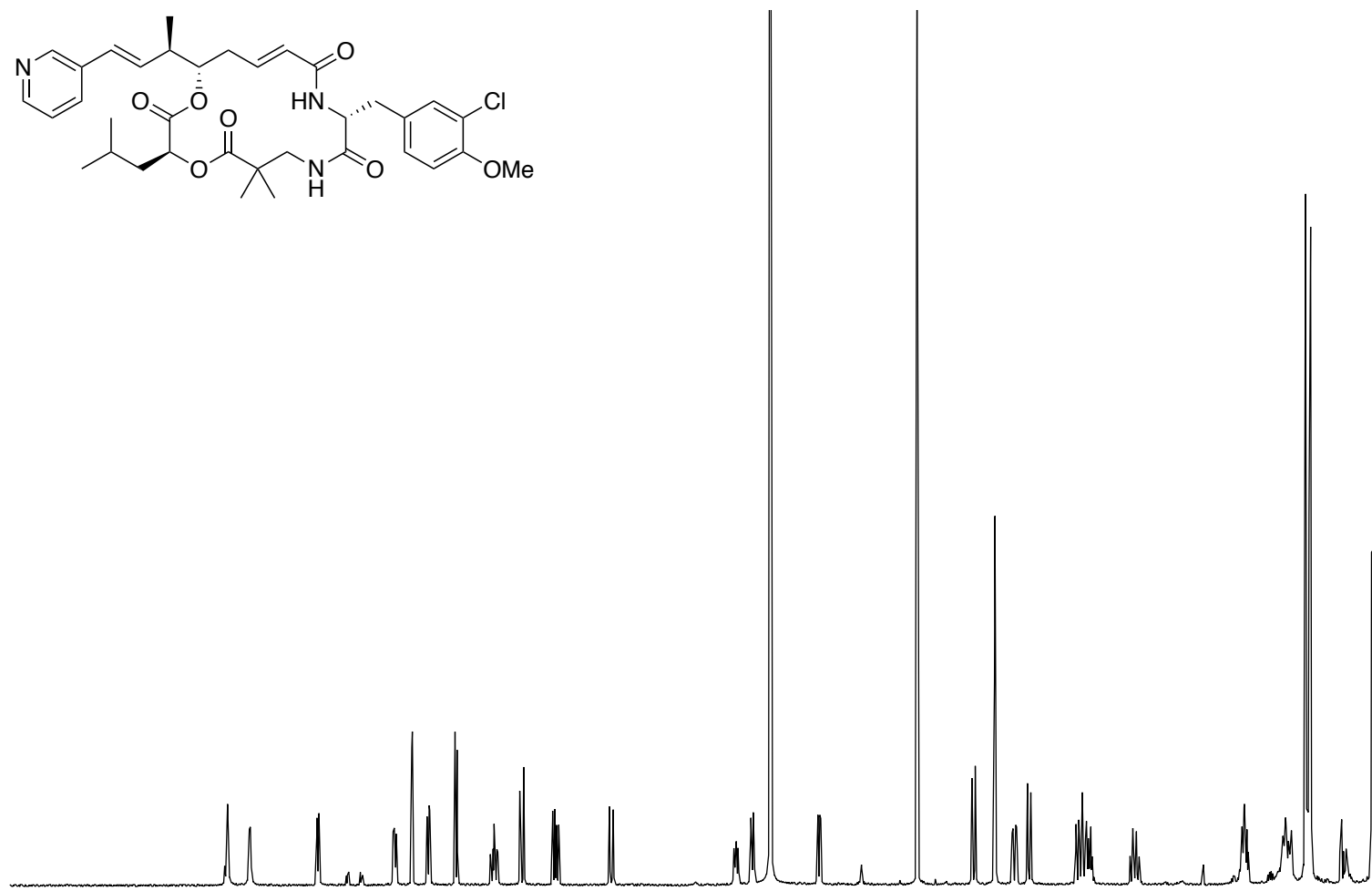

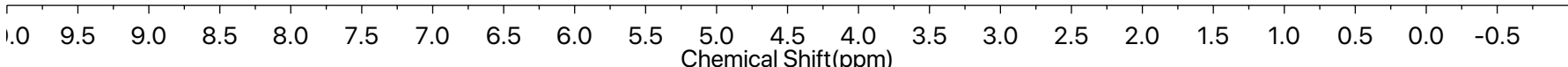

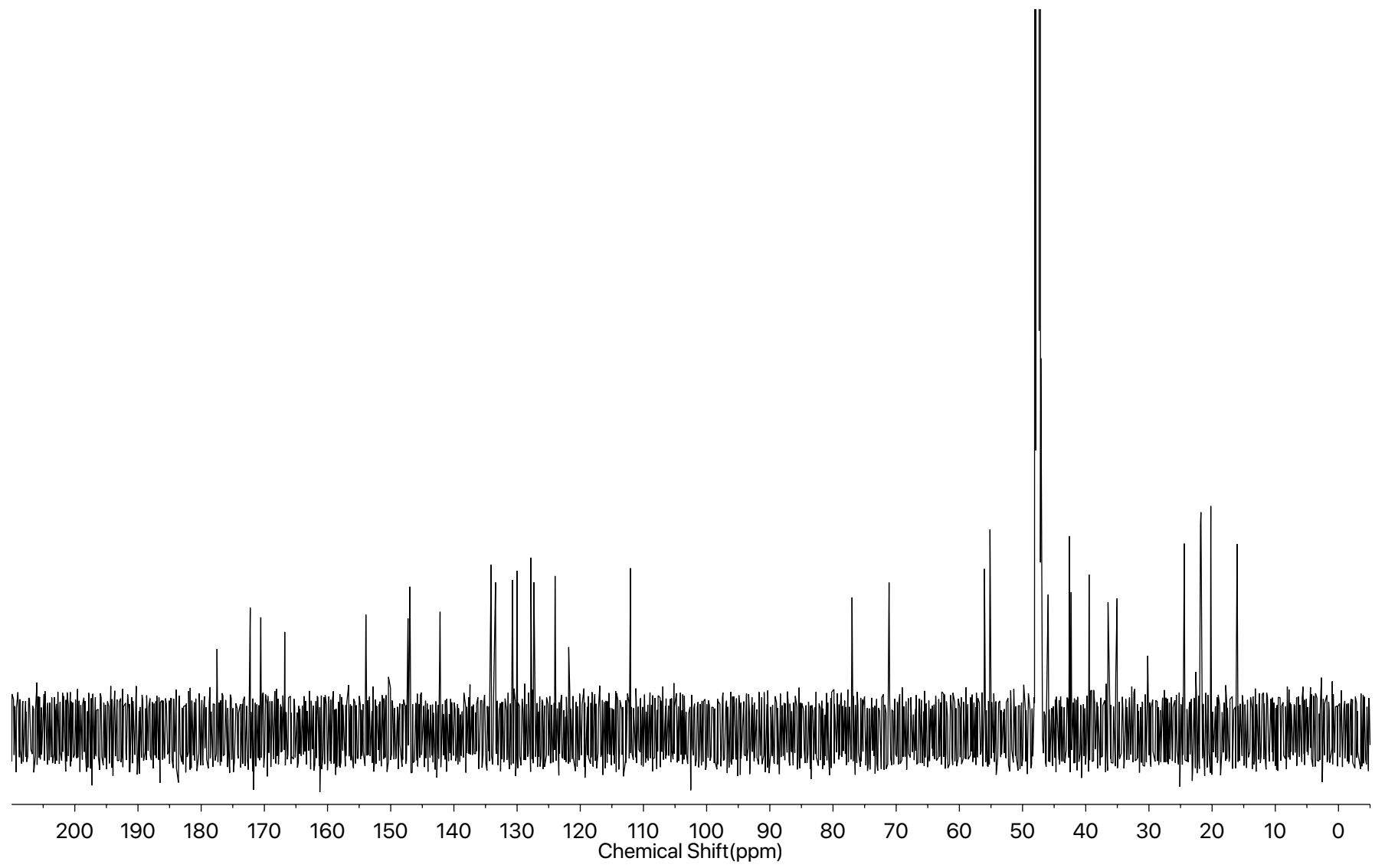



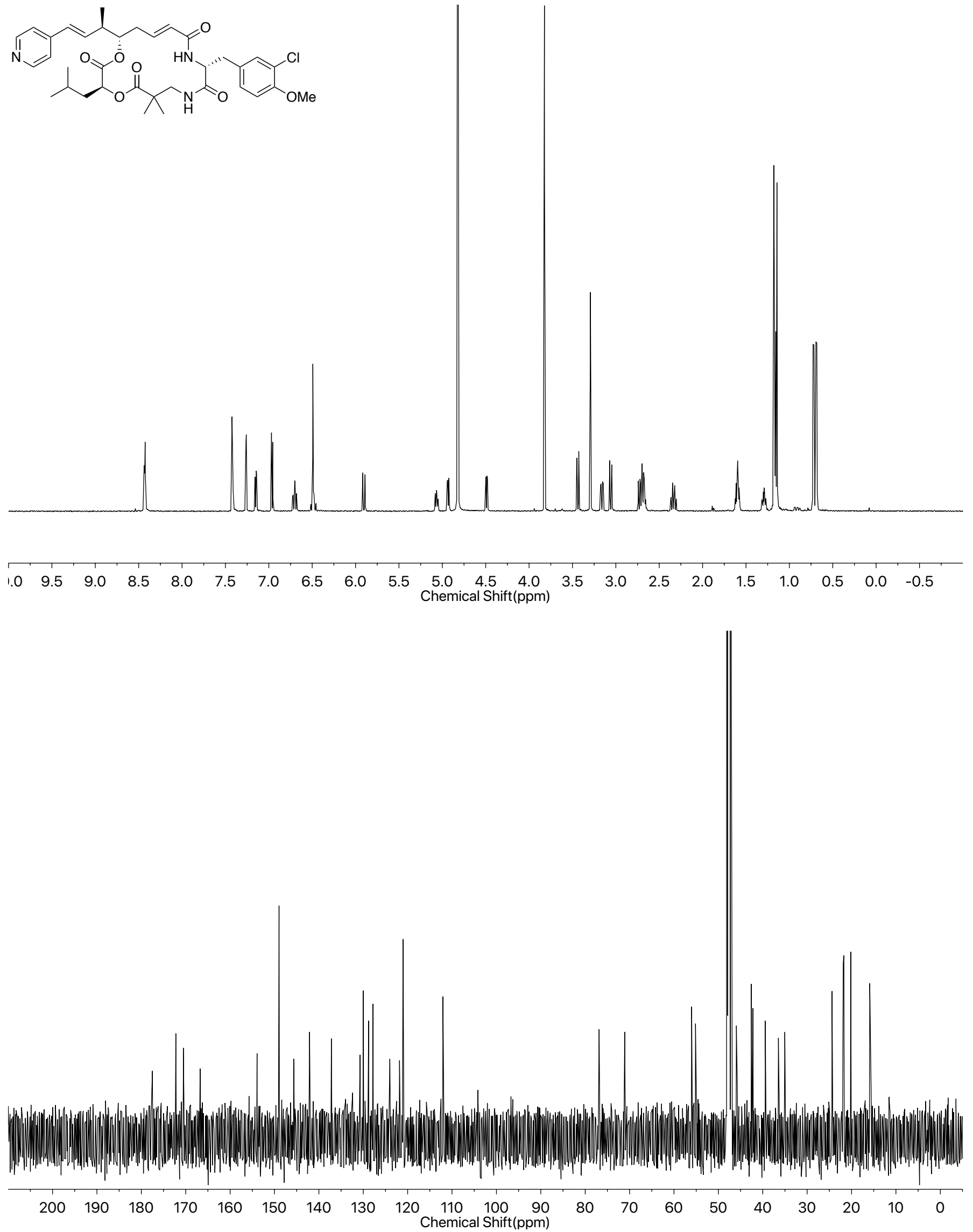
<smiles>COc1ccc(C[C@H](NC(=O)/C=C\CCC(C)/C=C/c2cnn(C)c2)C(=O)NCC(C)(C)C(=O)OC(CC(C)C)C(=O)O)cc1Cl</smiles>

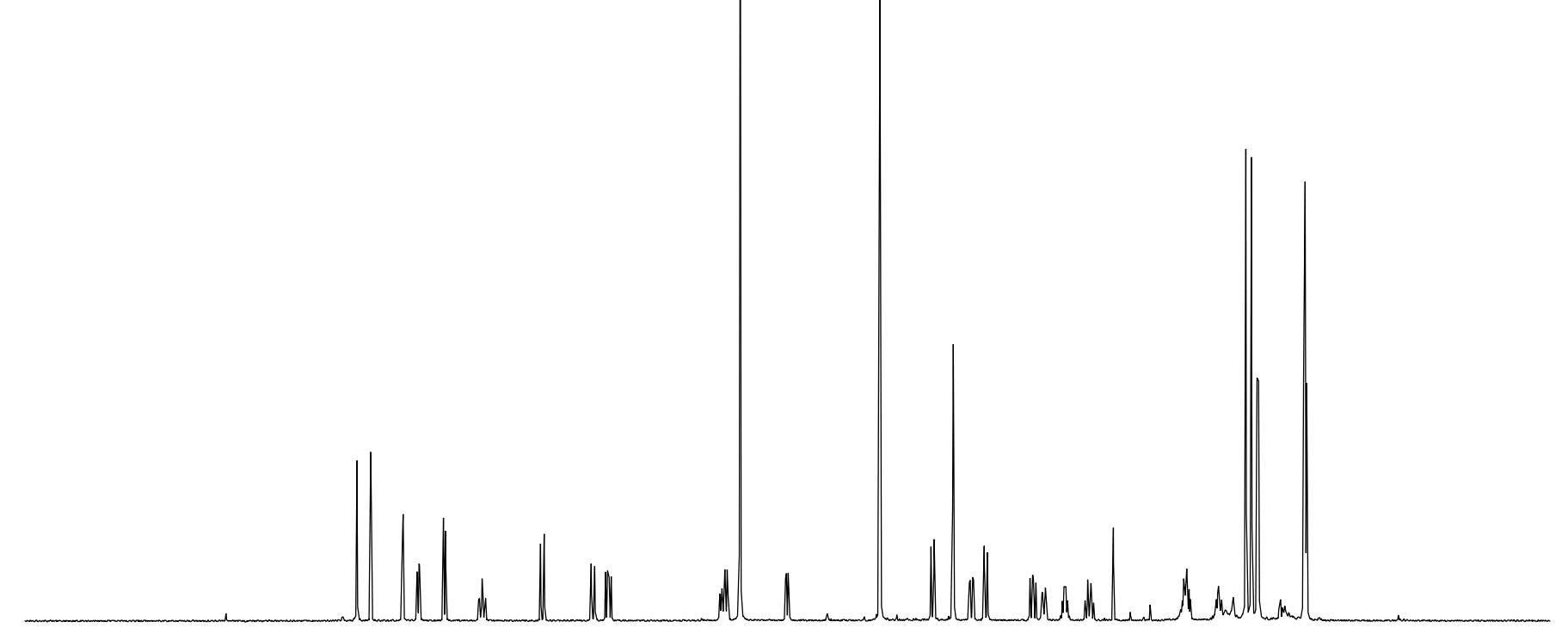

\begin{tabular}{llllllllllllllllllllllllll}
\hline .0 & 9.5 & 9.0 & 8.5 & 8.0 & 7.5 & 7.0 & 6.5 & 6.0 & 5.5 & $\begin{array}{c}5.0 \\
\text { Chemical Shift(ppm) }\end{array}$ & 4.5 & 3.0 & 2.5 & 2.0 & 1.5 & 1.0 & 0.5 & 0.0 & -0.5 & -1
\end{tabular}

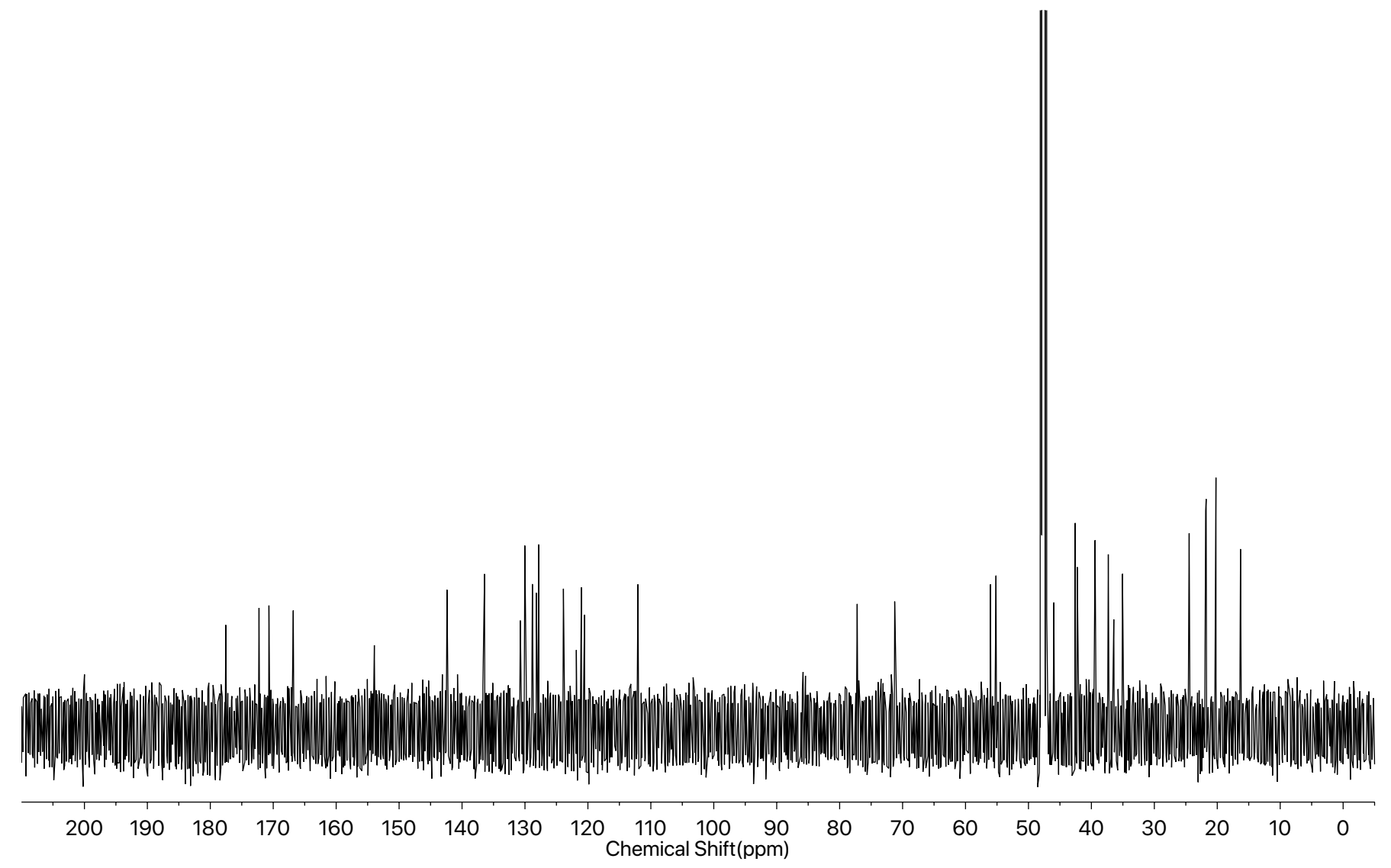




\section{$\underline{\text { References }}$}

[1] Tredwell, M., Tenza, K., Pacheco, M. C., and Gouverneur, V. (2005) Synthesis of novel enantiopure fluorinated building blocks from acyclic chiral allylsilanes, Org Lett 7, 4495-4497.

[2] Shankaraiah, G., Kumar, T. V., Reddy, G. V., Rao, J. M., and Babu, K. S. (2013) Stereoselective Synthesis of (-)-Pinidinone, Helv Chim Acta 96, 990-996.

[3] Gao, X., Han, H., and Krische, M. J. (2011) Direct Generation of Acyclic Polypropionate Stereopolyads via Double Diastereo- and Enantioselective Iridium-Catalyzed Crotylation of 1,3-Diols: Beyond Stepwise Carbonyl Addition in Polyketide Construction, J Am Chem Soc 133, 12795-12800.

[4] Phukan, P., Bauer, M., and Maier, M. E. (2003) Facile generation of alkenes and dienes from tosylates, Synthesis-Stuttgart, 1324-1328.

[5] Kotoku, N., Kato, T., Narumi, F., Ohtani, E., Kamada, S., Aoki, S., Okada, N., Nakagawa, S., and Kobayashi, M. (2006) Synthesis of 15,20-triamide analogue with polar substituent on the phenyl ring of arenastatin A, an extremely potent cytotoxic spongean depsipeptide, Bioorgan Med Chem 14, 7446-7457.

[6] Krishnamurthy, S. (1978) Rapid Reduction of Alkyl Tosylates with Lithium Triethylborohydride Convenient and Advantageous Procedure for Deoxygenation of Simple and Hindered Alcohols Comparison of Various Hydride Reagents, J Organomet Chem 156, 171-181.

[7] Morrill, C., and Grubbs, R. H. (2003) Synthesis of functionalized vinyl boronates via ruthenium-catalyzed olefin cross-metathesis and subsequent conversion to vinyl halides, $J$ Org Chem 68, 6031-6034.

[8] McCubbin, J. A., Maddess, M. L., and Lautens, M. (2006) Total synthesis of cryptophycin analogues via a scaffold approach, Org Lett 8, 2993-2996.

[9] Ghosh, A. K., and Bischoff, A. (2004) Asymmetric syntheses of potent antitumor macrolides cryptophycin B and arenastatin A, Eur J Org Chem, 2131-2141.

[10] Ghosh, A. K., and Swanson, L. (2003) Enantioselective synthesis of (+)-cryptophycin 52 (LY355703), a potent antimitotic antitumor agent, $\mathrm{J}$ Org Chem 68, 9823-9826.

[11] Buck, S. B., Huff, J. K., Himes, R. H., and Georg, G. I. (2004) Total synthesis and anti-tubulin activity of epi-c3 analogues of cryptophycin-24, Journal of medicinal chemistry 47, 3697-3699.

[12] Mast, C. A., Eissler, S., Stoncius, A., Stammler, H. G., Neumann, B., and Sewald, N. (2005) Efficient and versatile stereoselective synthesis of cryptophycins, Chemistry 11, 4667-4677.

[13] Beck, Z. Q., Aldrich, C. C., Magarvey, N. A., Georg, G. I., and Sherman, D. H. (2005) Chemoenzymatic synthesis of cryptophycin/arenastatin natural products, Biochemistry-Us 44, 13457-13466.

[14] Piubelli, L., Aliverti, A., Bellintani, F., and Zanetti, G. (1995) Spinach ferredoxin i: overproduction in Escherichia coli and purification, Protein Expr Purif 6, 298-304.

[15] Aliverti, A., Jansen, T., Zanetti, G., Ronchi, S., Herrmann, R. G., and Curti, B. (1990) Expression in Escherichia coli of ferredoxin:NADP+ reductase from spinach. Bacterial synthesis of the holoflavoprotein and of an active enzyme form lacking the first 28 amino acid residues of the sequence, Eur J Biochem 191, 551-555.

[16] Omura, T., and Sato, R. (1964) The Carbon Monoxide-Binding Pigment of Liver Microsomes. I. Evidence for Its Hemoprotein Nature, The Journal of biological chemistry 239, 2370-2378.

[17] Khatri, Y., Hannemann, F., Ewen, K. M., Pistorius, D., Perlova, O., Kagawa, N., Brachmann, A. O., Muller, R., and Bernhardt, R. (2010) The CYPome of Sorangium cellulosum So ce56 and Identification of CYP109D1 as a New Fatty Acid Hydroxylase, Chem Biol 17, 1295-1305.

[18] Williams, J. W., and Morrison, J. F. (1979) The kinetics of reversible tight-binding inhibition, Methods Enzymol 63, 437-467. 